\title{
Unsupervised learning for vascular heterogeneity assessment of glioblastoma based on magnetic resonance imaging: The Hemodynamic Tissue Signature
}

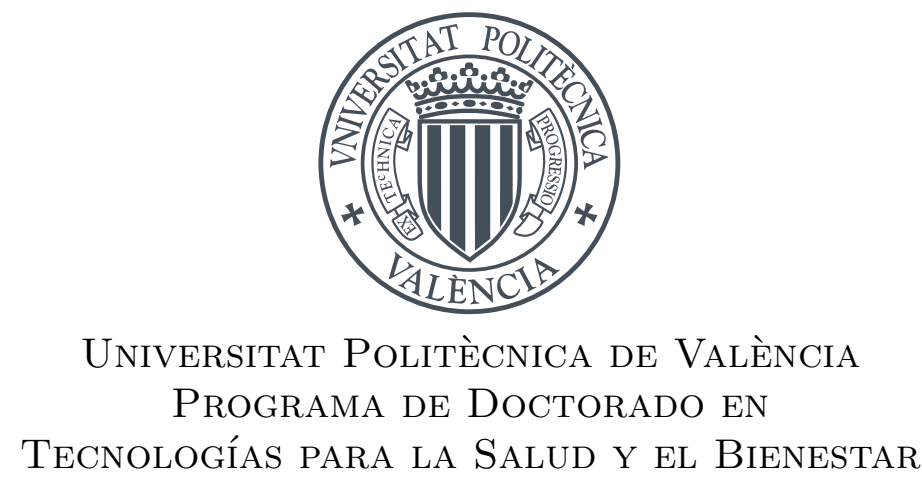

DOCTORAL THESIS

Presented by

Javier Juan Albarracín

Directed by

Dr. Juan M García-Gómez

Dr. Elies Fuster i Garcia

Valencia, Spain

March 2020 



\section{Agradecimientos Acknowledgements}

La finalización de esta tesis me brinda la oportunidad de reconocer a todas aquellas personas que, gracias al apoyo, el esfuerzo y la confianza incondicional que han depositado en mi, han hecho de este trabajo una realidad. A ellas quiero dedicar esta tesis.

En primer lugar, quiero expresar mi más profundo agradecimiento a mis directores de tesis: el Dr. Juan Miguel García Gómez y el Dr. Elies Fuster i Garcia. Hace más de 8 años que me abrieron las puertas del grupo de investigación IBIME y apostaron por mí para desarrollar la línea de investigación de pattern recognition en imagen médica. Hoy, los frutos del trabajo que llevamos realizando juntos durante todo este tiempo se ven reflejados en esta tesis que, de no haber sido por su certera dirección, su excelencia científica y su enorme calidad humana, no habría sido posible. Gracias por ofrecerme la oportunidad de trabajar a vuestro lado y de aprender de quienes considero referentes tanto a nivel profesional como personal.

Quiero agradecer también a todos los compañeros y amigos del grupo IBIME que me han acompañado durante todos estos años en los que he tenido la oportunidad de desarrollar mi carrera científica y mi vida personal. Todos ellos han sido parte activa de este camino, contribuyendo con sus ideas, reflexiones y conocimiento al desarrollo esta tesis. Gracias a los que ahora formáis parte del grupo: el Dr. Carlos Sáez, el Dr. Jose Enrique Romero, Marta Durá, Vicent Blanes, Mari Alvarez, Pablo Ferri y Ángel Sánchez; y a los que formasteis parte en el pasado: el Dr. Salvador Tortajada, Miguel Esparza, el Dr. Adrián Bresó, Germán García y Alfonso Pérez, así como al Dr. Jose Vicente Manjón Herrera por su inestimable ayuda y su inagotable pasión por la ciencia. Gracias por servir de apoyo ante cualquier obstáculo y por los insuperables buenos momentos y risas que compartimos siempre en cualquier momento.

La investigación de excelencia es hoy en día posible únicamente mediante la participación en proyectos de $\mathrm{I}+\mathrm{D}+\mathrm{i}$ tanto nacionales como internacionales, públicos o privados. En este sentido quiero agradecer a las diferentes instituciones y estructuras de financiación de investigación que han contribuido al desarrollo de esta tesis. En especial quiero agradecer a la Universitat Politècnica de València, donde he desarrollado toda mi carrera académica y científica, así como al Ministerio de Ciencia e Innovación, al Ministerio de Economía y Competitividad, a la Comisión Europea, al EIT Health Programme y a la fundación Caixa Impulse. También quiero agradecer a los hospitales y centros sanitarios que han participado en esta tesis aportando casos 
de estudio y acertados consejos para el buen desarrollo de este trabajo. Agradezco al Hospital Politècnico y Universitario La Fe, al Hospital de la Ribera, al Hospital de Manises, al Hospital Clínic de Barcelona, al Hospital Vall d'Hebrón, a l'Azienda Ospedaliero-Universitaria di Parma, al CHU de Liège y al Oslo University Hospital.

Por último, quiero dedicar especialmente esta tesis a mi familia. A mi madre María Ángeles, que siempre me ha inculcado los valores de lucha y esfuerzo, de responsabilidad y constancia. Tu incansable empeño en educarme en estos valores han hecho de mi la persona que soy hoy. A mi hermano Eduardo, al que cuantos más años pasan más admiro. Gracias por estar a mi lado en los momentos más difíciles y guiarme en muchos aspectos de mi vida. Te has convertido en un espejo en el que mirarme cada día. Y por último a mi padre Rafael, que siempre me ha enseñado el valor de la honestidad y el respeto, de la excelencia y el sacrificio. A veces la vida es más dura con quien menos se lo merece, y aún en los peores momentos has demostrado ser siempre ser un referente, para mí y para todos. No puedo sentirme más orgulloso de tener esta familia. 


\section{Abstract}

The future of medical imaging is linked to Artificial Intelligence (AI). The manual analysis of medical images is nowadays an arduous, error-prone and often unaffordable task for humans, which has caught the attention of the Machine Learning (ML) community. Magnetic Resonance Imaging (MRI), which constitutes the standard imaging technique for the diagnosis of many lethal diseases, provides us with a wide variety of rich representations of the morphology and behavior of lesions completely inaccessible without a risky invasive intervention. Nevertheless, harnessing the powerful but often latent information contained in MRI acquisitions is a very complicated task, which requires computational intelligent analysis techniques.

Central nervous system tumors are one of the most critical diseases studied through MRI. Specifically, glioblastoma represents a major challenge, as it remains a lethal cancer that, to date, lacks a satisfactory therapy. Of the entire set of characteristics that make glioblastoma so aggressive, a particular aspect that has been widely studied is its vascular heterogeneity. The strong vascular proliferation of glioblastomas, as well as their robust angiogenesis and extensive microvasculature heterogeneity have been claimed responsible for the high lethality of the neoplasm. Therefore, the study of these hallmarks is crucial to better understand the tumor's aggressiveness and design new effective therapies that improve patient prognosis.

This thesis focuses on the research and development of the Hemodynamic Tissue Signature (HTS) method: an unsupervised ML approach to describe the vascular heterogeneity of glioblastomas by means of perfusion MRI analysis. The HTS builds on the concept of habitats. A habitat is defined as a sub-region of the lesion with a particular MRI profile describing a specific physiological behavior. The HTS method delineates four habitats within the glioblastoma: the High Angiogenic Tumor (HAT) habitat, as the most perfused region of the enhancing tumor; the Low Angiogenic Tumor (LAT) habitat, as the region of the enhancing tumor with a lower angiogenic profile; the potentially Infiltrated Peripheral Edema (IPE) habitat, as the non-enhancing region adjacent to the tumor with elevated perfusion indexes; and the Vasogenic Peripheral Edema (VPE) habitat, as the remaining edema of the lesion with the lowest perfusion profile. The research and development of the HTS method has generated a number of contributions to this thesis.

First, in order to verify that unsupervised learning methods are reliable to extract MRI patterns to describe the heterogeneity of a lesion, a comparison among several structured and non-structured unsupervised learning methods was conducted for the task of high grade glioma segmentation. Additionally a generic postprocessing stage 
was also developed to automatically map each label of an unsupervised segmentation to a healthy or pathological tissue of the brain.

Second, a Bayesian unsupervised learning algorithm from the family of Spatially Varying Finite Mixture Models (SVFMMs) is proposed. The algorithm, named Non Local Spatially Varying Finite Mixture Model (NLSVFMM), successfully integrates a continuous Gauss-Markov Random Field (MRF) prior density weighted by the probabilistic Non Local Means (NLM) weighting function, to codify the idea that neighboring pixels tend to belong to the same semantic object. The proposed prior simultaneously enforces local smoothness on the segmentations, while preserves the edges and the structure between classes.

Third, the HTS method to describe the vascular heterogeneity of glioblastomas through the aforementioned habitats is presented. The HTS method has been applied to real cases, both in a local cohort of patients from a single-center, and in an international retrospective cohort of more than 180 patients from 7 European centers. A comprehensive evaluation of the method was conducted to measure the prognostic potential of the HTS habitats, as well as their stratification capabilities to identify populations with different prognosis. Statistically significant associations were found between most of HTS habitats and Overall Survival (OS) of patients, as well as significant differences were observed in survival rates of sub-populations divided according to HTS derived measurements.

Finally, the methods and technology developed in this thesis have been integrated into an online public open-access platform for its academic use. The ONCOhabitats platform is hosted at https://www.oncohabitats.upv.es, and provides two main services: 1) glioblastoma tissue segmentation, and 2) vascular heterogeneity assessment of glioblastomas by means of the HTS method. Both services, in addition to preprocessed images and segmentation maps, automatically generate a radiological report, summarizing the findings of the study. ONCOhabitats not only offers the scientific and medical community access to leading-edge algorithms for the analysis of these tumors, but gives access to its computational cluster capable to process about 300 cases per day.

The results of this thesis have been published in ten scientific contributions, including top-ranked journals and conferences in the areas of Medical Informatics, Statistics and Probability, Radiology \& Nuclear Medicine, Machine Learning and Data Mining and Biomedical Engineering. An industrial patent registered in Spain (ES201431289A), Europe (EP3190542A1) and EEUU (US20170287133A1) was also issued, summarizing the efforts of this thesis to generate tangible assets besides the academic revenue obtained from research publications. Finally, the methods, technologies and original ideas conceived in this thesis led to the foundation of ONCOANALYTICS CDX, a company framed into the business model of companion diagnostics for pharmaceutical compounds, thought as a vehicle to facilitate the industrialization of the ONCOhabitats technology. 


\section{Resumen}

El futuro de la imagen médica está ligado a la Inteligencia Artificial (IA). El análisis manual de imágenes médicas es hoy en día una tarea ardua, propensa a errores y a menudo inasequible para los humanos, que ha llamado la atención de la comunidad de Aprendizaje Automático (AA). La Imagen por Resonancia Magnética (IRM), que constituye la técnica de imagen estándar para el diagnóstico de muchas enfermedades letales, nos proporciona una amplia y rica variedad de representaciones de la morfología y el comportamiento de lesiones completamente inaccesibles sin una intervención invasiva arriesgada. Sin embargo, explotar la potente pero a menudo latente información contenida en las adquisiciones de IRM es una tarea muy complicada, que requiere técnicas de análisis computacional inteligente.

Los tumores del sistema nervioso central son una de las enfermedades más críticas estudiadas a través de IRM. Específicamente, el glioblastoma representa un gran desafío, ya que, hasta la fecha, continua siendo un cáncer letal que carece de una terapia satisfactoria. De todo el conjunto de características que hacen del glioblastoma un tumor tan agresivo, un aspecto particular que ha sido ampliamente estudiado es su heterogeneidad vascular. La fuerte proliferación vascular de los glioblastomas, así como su robusta angiogénesis y la extensa heterogeneidad de su microvasculatura han sido consideradas responsables de la alta letalidad de esta neoplasia. Por lo tanto, el estudio de estos factores es crucial para entender mejor la agresividad del tumor y diseñar nuevas terapias efectivas que mejoren el pronóstico del paciente.

Esta tesis se centra en la investigación y desarrollo del método Hemodynamic Tissue Signature (HTS): un método de aprendizaje no supervisado para describir la heterogeneidad vascular de los glioblastomas mediante el análisis de perfusión por IRM. El método HTS se basa en el concepto de hábitats. Un hábitat se define como una subregión de la lesión con un perfil particular de IRM, que describe un comportamiento fisiológico concreto. El método HTS delinea cuatro hábitats dentro del glioblastoma: el hábitat High Angiogenic Tumor (HAT), como la región más perfundida del tumor con captación de contraste; el hábitat Low Angiogenic Tumor (LAT), como la región del tumor con captación de contraste con un perfil angiogénico más bajo; el hábitat Infiltrated Peripheral Edema (IPE), como la región edematosa sin captación de contraste adyacente al tumor con índices de perfusión elevados; y el hábitat Vasogenic Peripheral Edema (VPE), como el edema restante de la lesión con el perfil de perfusión más bajo. La investigación y desarrollo del método HTS ha originado una serie de contribuciones enmarcadas en esta tesis.

En primer lugar, para verificar que los métodos de aprendizaje no supervisados son 
fiables a la hora de extraer patrones de IRM para describir la heterogeneidad de una lesión, se realizó una comparación entre varios métodos de aprendizaje estructurado y no estructurado no supervisados en la tarea de segmentación de gliomas de grado alto. Adicionalmente, se desarrolló un método genérico de postproceso para mapear automáticamente cada etiqueta de una segmentación no supervisada a un tejido sano o patológico del cerebro.

En segundo lugar, se ha propuesto un algoritmo de aprendizaje Bayesiano no supervisado dentro de la familia de los Spatially Varying Finite Mixture Models (SVFMMs). El algoritmo, llamado Non Local Spatially Varying Finite Mixture Model (NLSVFMM), integra con éxito un Gauss-Markov Random Field (MRF) continuo ponderado por la función probabilística Non Local Means (NLM) como densidad a priori del modelo, para codificar la idea de que los píxeles vecinos tienden a pertenecer al mismo objeto semántico. La probabilidad a priori propuesta refuerza simultáneamente la suavidad local en las segmentaciones, a la vez que preserva los bordes y la estructura entre clases.

En tercer lugar, se presenta el método HTS para describir la heterogeneidad vascular de los glioblastomas mediante los hábitats mencionados. El método HTS se ha aplicado a casos reales, tanto en una cohorte local de pacientes de un solo centro, como en una cohorte retrospectiva internacional de más de 180 pacientes de 7 centros europeos. Se llevó a cabo una evaluación exhaustiva del método para medir el potencial pronóstico de los hábitats, así como las capacidades de estratificación de los mismos para identificar poblaciones con pronósticos diferentes. Se encontraron asociaciones estadísticamente significativas entre la mayoría de los hábitats HTS y la supervivencia global de los pacientes, así como diferencias significativas en las tasas de supervivencia de subpoblaciones divididas según mediciones derivadas del HTS.

Finalmente, los métodos y la tecnología desarrollados en esta tesis se han integrado en una plataforma web online de acceso público para su uso académico. La plataforma ONCOhabitats se aloja en https://www.oncohabitats.upv.es, y ofrece dos servicios principales: 1) segmentación de tejidos de glioblastoma, y 2) evaluación de la heterogeneidad vascular de los glioblastomas mediante el método HTS. Ambos servicios, además de las imágenes preprocesadas y los mapas de segmentación, generan automáticamente un informe radiológico resumiendo los hallazgos del estudio. ONCOhabitats no sólo ofrece a la comunidad científica y médica acceso a algoritmos del estado del arte para el análisis de estos tumores, sino que también permite acceder a su clúster computacional, capaz de procesar cerca de 300 casos al día.

Los resultados de esta tesis han sido publicados en diez contribuciones científicas, incluyendo revistas y conferencias de primer nivel en las áreas de Informática Médica, Estadística y Probabilidad, Radiología y Medicina Nuclear, Aprendizaje Automático y Minería de Datos e Ingeniería Biomédica. También se emitió una patente industrial registrada en España (ES201431289A), Europa (EP3190542A1) y EEUU (US20170287133A1), que representa los esfuerzos de esta tesis para generar activos tangibles además de los méritos académicos obtenidos de las publicaciones de investigación. Finalmente, los métodos, tecnologías e ideas originales concebidas en esta tesis dieron lugar a la creación de ONCOANALYTICS CDX, una empresa enmarcada en el modelo de negocio de los companion diagnostics de compuestos farmacéuticos, pensado como vehículo para facilitar la industrialización de la tecnología ONCOhabitats. 


\section{Resum}

El futur de la imatge mèdica està lligat a la Intel-ligència Artificial (IA). L'anàlisi manual d'imatges mèdiques és hui dia una tasca àrdua, propensa a errors i sovint inassequible per als humans, que ha cridat l'atenció de la comunitat d'Aprenentatge Automàtic (AA). La Imatge per Ressonància Magnètica (IRM), que constitueix la tècnica d'imatge estàndard per al diagnòstic de moltes malalties letals, ens proporciona una àmplia i rica varietat de representacions de la morfologia i el comportament de lesions completament inaccessibles sense una intervenció invasiva arriscada. Tanmateix, explotar la potent però sovint latent informació continguda a les adquisicions de IRM esdevé una tasca molt complicada, que requereix tècniques d'anàlisi computacional intel-ligent.

Els tumors del sistema nerviós central són una de les malalties més crítiques estudiades a través de IRM. Específicament, el glioblastoma representa un gran repte, ja que, fins hui, continua siguent un càncer letal que manca d'una teràpia satisfactòria. Dintre del conjunt de característiques que fan del glioblastoma un tumor tan agressiu, un aspecte particular que ha sigut àmpliament estudiat és la seua heterogeneïtat vascular. La forta proliferació vascular dels glioblastomes, així com la seua robusta angiogènesi i l'extensa heterogeneïtat de la seua microvasculatura han sigut considerades responsables de l'alta letalitat d'aquesta neoplàsia. Per tant, l'estudi d'aquests factors esdevé crucial per entendre millor l'agressivitat del tumor i dissenyar noves teràpies efectives que milloren el pronòstic del pacient.

Aquesta tesi es centra en la recerca i desenvolupament del mètode Hemodynamic Tissue Signature (HTS): un mètode d'aprenentatge no supervisat per descriure l'heterogeneïtat vascular dels glioblastomas mitjançant l'anàlisi de perfusió per IRM. El mètode HTS es basa en el concepte d'hàbitats. Un hàbitat es defineix com una subregió de la lesió amb un perfil particular d'IRM, que descriu un comportament fisiològic concret. El mètode HTS delinea quatre hàbitats dins del glioblastoma: l'hàbitat High Angiogenic Tumor (HAT), com la regió més perfosa del tumor amb captació de contrast; l'hàbitat Low Angiogenic Tumor (LAT), com la regió del tumor amb captació de contrast amb un perfil angiogènic més baix; l'hàbitat Infiltrated Peripheral Edema (IPE), com la regió edematosa sense captació de contrast adjacent al tumor amb índexs de perfusió elevats, i l'hàbitat Vasogenic Peripheral Edema (VPE), com l'edema restant de la lesió amb el perfil de perfusió més baix. La recerca i desenvolupament del mètode HTS ha originat una sèrie de contribucions emmarcades a aquesta tesi.

En primer lloc, per verificar que els mètodes d'aprenentatge no supervisats són fiables a l'hora d'extraure patrons d'IRM per descriure l'heterogeneïtat d'una lesió, 
es va realitzar una comparació entre diversos mètodes d'aprenentatge estructurat i no estructurat no supervisats en la tasca de segmentació de gliomes de grau alt. Addicionalment, es va desenvolupar un mètode genèric de post-processament per mapejar automàticament cada etiqueta d'una segmentació no supervisada a un teixit sa o patològic del cervell.

En segon lloc, s'ha proposat un algorisme d'aprenentatge Bayesià no supervisat dintre de la família dels Spatially Varying Finite Mixture Models (SVFMMs). L'algorisme, anomenat Non Local Spatially Varying Finite Mixture Model (NLSVFMM), integra amb èxit un Gauss-Markov Random Field (MRF) continu ponderat per la funció probabilística Non Local Means (NLM) com a densitat a priori del model, per a codificar la idea que els píxels veïns tendeixen a pertànyer al mateix objecte semàntic. La probabilitat a priori proposada reforça simultàniament la suavitat local en les segmentacions, alhora que preserva les vores i l'estructura entre classes.

En tercer lloc, es presenta el mètode HTS per descriure l'heterogeneïtat vascular dels glioblastomas mitjançant els hàbitats esmentats. El mètode HTS s'ha aplicat a casos reals, tant en una cohort local de pacients d'un sol centre, com en una cohort retrospectiva internacional de més de 180 pacients de 7 centres europeus. Es va dur a terme una avaluació exhaustiva del mètode per mesurar el potencial pronòstic dels hàbitats, així com les capacitats d'estratificació dels mateixos per identificar poblacions amb pronòstics diferents. Es van trobar associacions estadísticament significatives entre la majoria dels hàbitats HTS i la supervivència global dels pacients, així com diferències significatives en les taxes de supervivència de sub-poblacions dividides segons mesuraments derivats de l'HTS.

Finalment, els mètodes i la tecnologia desenvolupats en aquesta tesi s'han integrat en una plataforma web online d'accés públic per al seu ús acadèmic. La plataforma ONCOhabitats s'allotja en https://www.oncohabitats.upv.es, i ofereix dos serveis principals: 1) segmentació dels teixits del glioblastoma, i 2) avaluació de l'heterogeneïtat vascular dels glioblastomes mitjançant el mètode HTS. Ambdós serveis, a més de les imatges preprocessades i els mapes de segmentació, generen automàticament un informe radiològic resumint els descobriments de l'estudi. ONCOhabitats no sols ofereix a la comunitat científica i mèdica accés a algorismes de l'estat de l'art per l'anàlisi d'aquests tumors, sinó que també permet accedir al seu clúster computacional, capaç de processar prop de 300 casos al dia.

Els resultats d'aquesta tesi han sigut publicats en deu contribucions científiques, incloent revistes i conferències de primer nivell a les àrees d'Informàtica Mèdica, Estadística i Probabilitat, Radiologia i Medicina Nuclear, Aprenentatge Automàtic i Mineria de Dades i Enginyeria Biomèdica. També es va emetre una patent industrial registrada a Espanya (ES201431289A), Europa (EP3190542A1) i els EUA (US20170287133A1), que representa els esforços d'aquesta tesi per generar actius tangibles a més dels mèrits acadèmics obtinguts de les publicacions d'investigació. Finalment, els mètodes, tecnologies i idees originals concebudes en aquesta tesi van donar lloc a la creació d'ONCOANALYTICS CDX, una empresa emmarcada en el model de negoci dels companion diagnostics de compostos farmacèutics, pensat com a vehicle per facilitar la industrialització de la tecnologia ONCOhabitats. 


\section{Glossary}

\section{Mathematical notation}

X Random variable

$x \quad$ Particular realization of the random variable $X$

$p(X) \quad$ Marginal probability density function of the random variable $X$

$p(X, Y) \quad$ Joint probability density function of two random variables $X$ and $Y$

$p(X \mid Y) \quad$ Conditional probability density function of the random variable $X$ conditioned to $Y$

$\Theta \quad$ Vector of parameters of a probability density function

$\hat{\Theta} \quad$ Optimal vector of parameters under some optimization criteria

$\tilde{\Theta} \quad$ Initial guess of a vector of parameters

$p(X ; \boldsymbol{\Theta}) \quad$ Probability density function of the random variable $X$ subject to $\Theta$

$\mathcal{L}(\boldsymbol{\Theta} ; X) \quad$ Likelihood function of random variable $X$ given parameter vector $\Theta$

$\mathbf{x} \quad$ Column vector $\mathbf{x}$

$\mathbf{x}^{T} \quad$ Transpose of $\mathbf{x}$

$\mathbb{E}[p(X)] \quad$ Expectation of probability density function $p(X)$

$\mathbb{E}_{p(Y)}[p(X)] \quad$ Conditional expectation of probability density function $p(X)$ subject to $p(Y)$

$\mathbb{R}^{D} \quad D$-dimensional space of real numbers

$\frac{\partial x}{\partial t} \quad$ Partial derivative of variable $x$ with respect to variable $t$

$\mathcal{M}^{i} \quad$ Set of neighbors of the $i^{t h}$ observation

$\otimes \quad$ Convolution product

$\delta(\cdot) \quad$ Dirac delta function

$\|\cdot\| \quad$ Euclidean norm

$\{\cdot\} \quad$ Set of elements 


\section{Acronyms}

AI Artificial Intelligence

AIF Arterial Input Function

ANN Artificial Neural Network

ANTs Advanced Normalization Tools

ASL Arterial Spin Labeling

BDSLab Biomedical Data Science Laboratory

BRATS BRAin Tumor Segmentation

CAE Convolutional AutoEnconder

CBF Cerebral Blood Flow

CBICA Center for Biomedical Image Computing and Analytics

CBV Cerebral Blood Volume

cdf cumulative distribution function

CDSS Clinical Decision Support System

CDx Companion Diagnostic

CNN Convolutional Neural Network

CSF Cerebro-Spinal Fluid

CRF Conditional Random Field

DCAGMRF Directional Class-Adaptive Gauss-Markov Random Field

DCE Dynamic Contrast Enhanced

DCM Dirichlet Compound Multinomial

DL Deep Learning

DSC Dynamic Susceptibility Contrast

DTI Diffusion Tensor Imaging

DWI Diffusion Weighted Imaging

EGFR Epidermal Growth Factor Receptor

EM Expectation-Maximization

ET Enhancing Tumor

FLAIR Fluid Attenuation Inversion Recovery

FMM Finite Mixture Model 
FSE Fast Spin Echo

GBCA Gadolinium-Based Contrast Agent

GM Grey Matter

GMM Gaussian Mixture Model

HAC Hierarchical Agglomerative Clustering

HAT High Angiogenic Tumor

HMRF Hidden Markov Random Field

HR Hazard Ratio

HTS Hemodynamic Tissue Signature

HUPLF Hospital Universitario y Politécnico La Fe

ICBM International Consortium of Brain Mapping

i.i.d. independent and identically distributed

IPE Infiltrated Peripheral Edema

LAT Low Angiogenic Tumor

MAP Maximum A Posteriori

MICCAI Medical Image Computing and Computer Assisted Intervention

ML Machine Learning

MLE Maximum Likelihood Estimation

MLP Multi-Layer Perceptron

MNI Montreal Neurological Institute

MPRAGE Magnetization-Prepared Rapid Acquisition with Gradient Echo

MR Magnetic Resonance

MRF Markov Random Field

MRI Magnetic Resonance Imaging

MRSI Magnetic Resonance Spectroscopy Imaging

MTT Mean Transit Time

NLM Non Local Means

NLSVFMM Non Local Spatially Varying Finite Mixture Model

NMR Nuclear Magnetic Resonance

OS Overall Survival 
PCA Principal Component Analysis

PD Proton Density

PFS Progression Free Survival

PCT Patent Cooperation Treaty

pdf probability density function

pmf probability mass function

PWI Perfusion Weighted Imaging

qMRI Quantitative Magnetic Resonance Imaging

rCBV relative Cerebral Blood Volume

rCBF relative Cerebral Blood Flow

RF Random Forest

RI Rand Index

RMSE Root Mean Squared Error

ROI Region Of Interest

SaaS Software as a Service

SAR Simultaneous Auto-Regressive

SOM Self-Organizing Map

SVD Singular Value Decomposition

SVFMM Spatially Varying Finite Mixture Model

SVM Support Vector Machine

TC Tumor Core

TCIA The Cancer Imaging Archive

TE Echo Time

TR Repetition Time

UAB University of Alabama at Birmingham

UPGMA Unweighted Pair Group Method with Arithmetic mean

UPV Universitat Politècnica de València

VPE Vasogenic Peripheral Edema

WHO World Health Organization

WM White Matter

WT Whole Tumor 


\section{Contents}

Abstract $\quad$ iii

Resumen $\quad$ v

Resum vii

Glossary $\quad$ ix

1 Introduction $\quad 1$

1.1 Motivation . . . . . . . . . . . . . . . . . . 1

1.2 Research questions and objectives . . . . . . . . . . . . . . 3

1.3 Thesis contributions . . . . . . . . . . . . . . 5

1.3.1 Main contributions . . . . . . . . . . . . . 5

1.3.2 Scientific publications . . . . . . . . . . . . . . 7

1.3.3 Software . . . . . . . . . . . . . . . . . . 9

1.3.4 Clinical studies . . . . . . . . . . . . . . . . 10

1.3 .5 Patents . . . . . . . . . . . . . . . . 10

1.3.6 Transference . . . . . . . . . . . . . . . . . . . . . . . . . . . . . . . 11

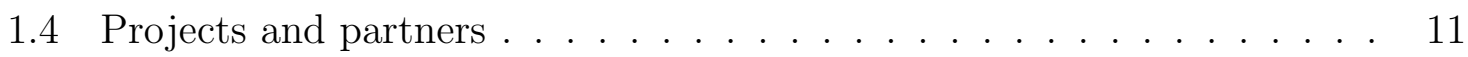

1.5 Thesis outline . . . . . . . . . . . . . . . . . 13

2 Rationale $\quad 17$

2.1 Glioblastoma . . . . . . . . . . . . . . . . . . . 17

2.2 Magnetic Resonance Imaging _ . . . . . . . . . . . . . . . . . . . . . . . . . . . . . 21

2.2.1 Anatomical MRI . . . . . . . . . . . . . . . . . . . 24

2.2.2 Quantitative MRI . . . . . . . . . . . . . . . 25

2.3 Probability theory . . . . . . . . . . . . . . . . 30

2.3.1 Basic concepts . . . . . . . . . . . . . . . 30

2.3.2 Probability distributions . . . . . . . . . . . . . . . . . . . . . . . . . . . 34

2.3.3 Parameter estimation . . . . . . . . . . . . . . . . . 39

2.4 Mixture Models . . . . . . . . . . . . . . . . . . . . . . . . . 43

2.4.1 Finite Mixture Model . . . . . . . . . . . . . . . . . . . 43

2.4.2 Spatially Varying Finite Mixture Model . . . . . . . . . . . . . . 48

2.4.3 DCM-Spatially Varying Finite Mixture Models . . . . . . . . . . 53

2.5 Deep Learning . . . . . . . . . . . . . . . . . . . . . . . . . . . . . 57

2.5.1 Artificial Neural Networks . . . . . . . . . . . . . . 58 
2.5.2 Convolutional Neural Networks _. . . . . . . . . . . . . 66

3 Comparative study of unsupervised learning algorithms for glioblas$\begin{array}{ll}\text { toma segmentation } & 73\end{array}$

3.1 Introduction . . . . . . . . . . . . . . . . . . . . . . . 74

3.2 Materials . . . . . . . . . . . . . . . . 76

3.3 Methods . . . . . . . . . . . . . . . . . . . 77

3.3.1 MRI preprocessing . . . . . . . . . . . . . 77

3.3.2 Feature Extraction and Dimensionality Reduction . . . . . . . . 80

3.3.3 Unsupervised voxel classification . . . . . . . . . . . . . . . 80

3.3.4 Automated pathological label identification . . . . . . . . . . 88

3.3.5 Evaluation . . . . . . . . . . . . . . . . . . . . . . 91

3.4 Results . . . . . . . . . . . . . . . . . . . . 92

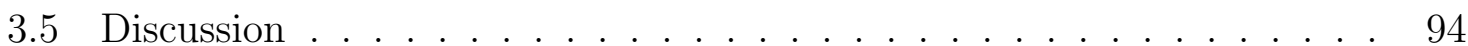

4 Non Local Spatially Varying Finite Mixture Models for unsupervised $\begin{array}{lr}\text { image segmentation } & 99\end{array}$

4.1 Introduction . . . . . . . . . . . . . . . . . . . . . . 100

4.2 Background on Spatially Varying Finite Mixture Models . . . . . . . . 101

4.3 Background on Probabilistic Non Local Means . . . . . . . . . . . . . . 102

4.4 The Non Local Spatially Variant Finite Mixture Model . . . . . . . . . 103

4.5 Experimental results . . . . . . . . . . . . . . . . 106

4.5.1 Evaluation on the BRATS 2013 high grade glioma dataset . . . 106

4.5.2 Evaluation on the Berkeley Segmentation Dataset . . . . . . . . 108

4.5.3 Evaluation of computational time requirements . . . . . . . . 110

4.6 Discussion . . . . . . . . . . . . . . . . . . . . . 111

5 Vascular heterogeneity assessment of glioblastoma through the Hemodynamic Tissue Signature 115

5.1 Introduction . . . . . . . . . . . . . . . . . 116

5.2 Materials . . . . . . . . . . . . . . . . . . 117

5.2.1 Patient selection . . . . . . . . . . . . . . . 117

5.2 .2 Magnetic Resonance Imaging . . . . . . . . . . . . . . . . . 118

5.3 Methods . . . . . . . . . . . . . . . . . . . . . 119

5.3.1 Quantification of DSC parametric maps . . . . . . . . . . . . 119

5.3.2 Enhancing tumor and edema segmentation . . . . . . . . . . . 119

5.3.3 Hemodynamic Tissue Signature habitats . . . . . . . . . . . . 120

5.3 .4 Statistical analysis . . . . . . . . . . . . . . . . . . . 121

5.4 Results . . . . . . . . . . . . . . . . . . . . . . . . 123

5.5 Discussion . . . . . . . . . . . . . . . . . . . . . . 128

6 Multi-center international validation of the Hemodynamic Tissue Signature for glioblastoma

6.1 Introduction . . . . . . . . . . . . . . . . . . . . . 133

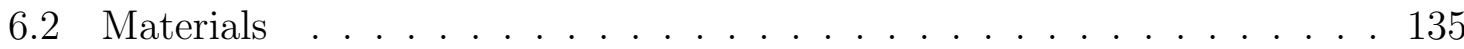

6.2 .1 Patient selection . . . . . . . . . . . . . . 135 
6.2 .2 Magnetic Resonance Imaging . . . . . . . . . . . . . . . . . . 136

6.3 Methods . . . . . . . . . . . . . . . . . . 136

6.3.1 Vascular heterogeneity assessment based on HTS habitats . . . 136

6.3.2 Association among OS and HTS markers - whole cohort study . 138

6.3.3 Association among OS and HTS markers - inter-center study . . 138

6.4 Results . . . . . . . . . . . . . . . . . . . . . . 138

6.4.1 Association among OS and HTS markers - whole cohort study . 138

6.4.2 Association among OS and HTS markers - inter-center study . . 139

6.5 Discussion . . . . . . . . . . . . . . . . . . . . 142

7 ONCOhabitats: A system for glioblastoma heterogeneity assessment $\begin{array}{lr}\text { through Magnetic Resonance Imaging } & 147\end{array}$

7.1 Introduction . . . . . . . . . . . . . . . . . . . . . 148

7.2 Materials . . . . . . . . . . . . . . . . . . . . . 149

7.3 Methods . . . . . . . . . . . . . . . . . . . 150

7.3.1 High-grade glioma segmentation service . . . . . . . . . . . . 151

7.3.2 Glioblastoma vascular heterogeneity assessment service . . . . . 152

7.3.3 Clinical report . . . . . . . . . . . . . . . . 153

7.3.4 ONCOhabitats system . . . . . . . . . . . . . 154

7.4 Results . . . . . . . . . . . . . . . . . . . . 160

7.4.1 High-grade glioma segmentation service . . . . . . . . . . . . . . 161

7.4.2 Glioblastoma vascular heterogeneity assessment service . . . . . 161

7.5 Discussion . . . . . . . . . . . . . . . . . . . 162

8 Concluding remarks and recommendations $\quad \mathbf{1 6 5}$

8.1 Concluding remarks . . . . . . . . . . . . . . . . . 165

8.2 Recommendations . . . . . . . . . . . . . . . . . . 167

$\begin{array}{ll}\text { Bibliography } & 171\end{array}$ 


\section{Chapter 1}

\section{Introduction}

\subsection{Motivation}

Medical imaging has widely proven to be an essential tool for modern medicine. The ability to non-invasively visualize in-vivo representations of the interior of the human body constituted an unprecedented breakthrough in the diagnosis, prognosis and follow-up processes of the diseases (McRobbie et al, 2007). The first medical image acquisition dates back to 1895 with the discovery of X-rays (Röntgen, 1898), however it was not until the end of the 20th century that medical imaging took a qualitative leap with the development of the MRI (Damadian, 1971; Lauterbur, 1973, 1974; Mansfield, 1977). Over the years, medical imaging has evolved rapidly, reaching sophisticated techniques capable of quantifying large amounts of information of the anatomy and functionality of human tissues. Consequently, the analysis of such complex information has become a specialized discipline that requires advanced computational techniques to make the most of the knowledge contained therein.

In this context, ML emerges as a solid candidate for medical image analysis. ML is an application of AI that provides systems the ability to learn and identify complex patterns in multi-dimensional data, and perform specific tasks without being explicitly programmed. The beginnings of ML in medical imaging dates back to the middle of the 20th century with the birth of the expert systems (Russell and Norvig, 2016). From that moment on, an unstoppable proliferation of ML systems for multitude of clinical problems took place, reaching its first hype cycle peak at the end of 20th century. Today, with the advent of the Deep Learning (DL) techniques, medical image analysis is undergoing a new deep revolution that is settling the ML as an indisputable instrument in the modern clinical practice.

However, ML has historically addressed medical problems from the perspective of automatizing arduous complex tasks for humans. Tied to medical imaging, ML has successfully addressed complicated problems such as identifying and delineating abnormal tissues in multiple images or classifying and grading lesions from medical acquisitions (Azuaje, 2019; Levine et al, 2019; Kann et al, 2019). Supervised learning, which is a family of techniques under the ML umbrella, has led this approach with unquestionable effectiveness. However, due to its learning nature, supervised learning is only able to address problems where humans already know the answer (Duda 
et al, 2000). This family of techniques builds models by means of learning the relations between tuples of 〈input data, desired output〉, which necessarily requires that all algorithm's possible outputs are explicitly known. This approach, without underestimating its unquestionable usefulness, reduces the ML to an instrument for solving and automatizing tasks with already well-known targets, with the added value of high accuracy, repeatability and reliability.

On the contrary, we would like ML to help us to discover new knowledge from the medical data beyond what humans can devise. At this point unsupervised learning arises as a tailor-made solution to this purpose. Unsupervised learning, unlike supervised learning, inspects unlabeled data for hidden patterns and inner relationships that describe the latent structure of the data (Bishop, 2006). This alternative approach has the intrinsic capability to discover new knowledge from the data in the form of new hypothesis about the structure and arrangement of the information. In this sense, unsupervised learning should assume a relevant role in medical imaging and must drive ML to serve not only as a tool for automating complex processes, but also as an instrument for exploring and extracting hidden knowledge from medical images.

A canonical example in which the convergence of medical imaging and ML must be reoriented towards the discovery of new knowledge is the study of highly aggressive heterogeneous tumors (Rudie et al, 2019; Tandel et al, 2019). Specifically, glioblastoma tumor represents a major challenge, as it remains a lethal tumor that, to date, lacks a satisfactory therapy, presenting one of the poorest prognosis among all human cancers, with a median survival time of 12-15 months despite aggressive treatment (Jain, 2018). Since the introduction of the Stupp treatment (Stupp et al, 2005) in 2005, there have been no significant changes in the therapies that have led to an improvement in patient prognosis. Therefore, the blockbuster model of "the same treatment for all" is considered to be depleted. In this regard, efforts today must be placed into the extraction of new knowledge from medical data that allow us to move towards new personalized more effective therapies.

One of the reasons believed to be behind this tumor's malignancy is its highly heterogeneous nature (Soeda et al, 2015). Glioblastomas are malignant masses characterized by hyper-cellularity, pleomorphism, micro-vascular proliferation and high necrosis mitotic activity (Gladson et al, 2010). Particularly, vascular heterogeneity has been largely studied since it is considered crucial for glioblastoma propagation and survival (Das and Marsden, 2013). Glioblastoma presents strong abnormal vascular proliferation, robust angiogenesis, and extensive micro-vasculature heterogeneity (Kargiotis et al, 2006), which have been shown to have a direct effect on prognosis (Hardee and Zagzag, 2012). Therefore, the early assessment of the heterogeneous vascular architecture of the tumor is thought to provide important information to improve and design new therapies.

However, measuring vascular heterogeneity from medical images is currently an uncertain task. There is no a consensus nor an accepted method to assess it. Consequently, there are no imaging protocols nor tools that includes such information in the clinical routine, hence ignoring this valuable knowledge in the management of the disease. Likewise, there are no expert manual annotations nor medical imaging datasets 
from where supervised ML algorithms can learn models. Such an undefined problem provides an excellent opportunity to apply unsupervised learning as an instrument for exploring new knowledge about the vascular profile of the tumor.

This thesis confronts all the factors mentioned above. The thesis focuses on the conjugation of unsupervised ML techniques on medical imaging data to non-invasively measure and describe the vascular heterogeneity of glioblastomas. This premise has established the main motivation and goals of this thesis, leading to the following research questions and objectives.

\subsection{Research questions and objectives}

The application of unsupervised ML techniques to medical images for brain tumor analysis poses a number of challenges that need to be addressed. First of all, by definition, unsupervised learning is a more open and undefined task than supervised learning. Unlike the latter, unsupervised learning solutions generally lack of semantic meaning. On the contrary, they usually consist of an interpretation of the data structure in terms of subgroups that share similar patterns with each other. Therefore, these algorithms require intelligent strategies, both during training and in the posterior analysis of the solution, to guide them towards the extraction of useful, consistent and interpretable knowledge.

Tied to the characterization of the vascular heterogeneity of glioblastomas, unsupervised learning methods need to be guided to find hidden patterns in perfusion medical images that are consistent with physiologically plausible hypotheses. In this sense, these unsupervised methods must serve not only as a mere algorithms for the characterization of the vascular profile of the tumor, but to tools to measure advanced imaging biomarkers that could give clues about the underlying physiological process taking place in the tumor.

Under a more technical point of view, learning patterns from imaging data also poses important challenges that must take into a account. Imaging data presents patterns of local regularity and spatial redundancy that suggest that they cannot be assumed to be independent and identically distributed (i.i.d.). Robust image analysis algorithms - both supervised and unsupervised learning - must consider this structured nature of the images and must include mechanisms to take advantage from this latent information.

As a consequence the next research questions are proposed in this thesis:

RQ1 Is unsupervised learning an adequate and reliable solution to extract valuable knowledge from complex MRI data?

RQ2 Can we contribute to the unsupervised learning family with new structured prediction algorithms to improve the extraction of knowledge from images?

RQ3 Can we contribute to a better understanding of the vascular heterogeneity of the glioblastoma by means of an advanced analysis of MRI through unsupervised learning methods? 
RQ4 Can useful measurements be obtained from the vascular heterogeneity description of glioblastoma that provide meaningful information on relevant patient's clinical outcomes?

RQ5 Can the unsupervised learning method to describe the vascular heterogeneity of glioblastomas be part of a medical imaging software for a complete analysis of the tumor through MRI?

The research work conducted in this thesis aims to provide solutions to these questions by means of theoretical and empirically validated scientific methods applied to the study of the vascular heterogeneity of glioblastomas by MRI. To this end the following objectives were defined:

O1 Review of the state-of-the-art in unsupervised learning algorithms, especially focused on image oriented algorithms and paying special attention to those applied to the analysis of brain tumor MRI images.

O2 Evaluate the feasibility of unsupervised learning algorithms to analyze and extract knowledge from MRI data.

O3 Develop a new unsupervised learning algorithm to exploit the structured nature of the images and take advantage from the spatial redundancy and local information contained therein.

O4 Develop a methodology based on image processing and unsupervised learning algorithms to detect and describe the vascular heterogeneity of glioblastoma through MRI.

O5 Validate the vascular heterogeneity assessment method on real clinical routine MRI, by exploring associations between the proposed heterogeneity description and relevant clinical outcomes of the patient.

O6 Implement a reliable tool capable of providing an advanced state-of-the-art analyses for glioblastoma MRI studies, including the methods to describe the vascular heterogeneity profile of the tumor.

The proposed objectives enclose the main goal of this thesis: the study of unsupervised ML techniques for medical imaging data to non-invasively describe the vascular heterogeneity of glioblastomas. Such goal can also be decomposed in two strands depending on the research scope: the technical goal, which aims to consolidate the unsupervised learning as a reliable tool for the future of medical image analysis; and the clinical goal, which intends to non-invasively describe the vascular heterogeneity of glioblastoma trough MRI to improve tumor understanding and clinical decision making. In this sense, the following scientific contributions support the achievement of the proposed objectives. 


\subsection{Thesis contributions}

This section presents the main contributions of this thesis. First, a summary of the most relevant aspects of each contribution is presented. Next, the scientific publications in high impact journals and conferences are listed. Finally, the technological and software results, as well as clinical studies, industrial patents and transfer actions are compiled.

\subsubsection{Main contributions}

\section{C1 - Comparative study of unsupervised learning algorithms for glioblas- toma segmentation}

In this study, a comparison of unsupervised learning algorithms, including structured and non-structured methods was performed for the task of high grade glioma segmentation. The study describes the statistical model underlying each algorithm and also proposes a general post-processing stage to identify which classes of an unsupervised segmentation correspond to pathological or healthy tissues. An independent evaluation of the performance of the unsupervised learning algorithms was carried out in a public real dataset, which demonstrated the capability of unsupervised learning to extract relevant knowledge from MRI data. This work was published in the journal contribution P1 (Juan-Albarracín et al, 2015b) and presented in the conference P2 (Juan-Albarracín et al, 2015a).

\section{C2 - An unsupervised learning algorithm for structured prediction}

A new variant of the Spatially Varying Finite Mixture Models (SVFMMs) family is proposed in this thesis. The algorithm, named Non Local Spatially Varying Finite Mixture Model (NLSVFMM), successfully merges the SVFMMs with the Non Local Means (NLM) framework, proposing a continuous Markov Random Field (MRF) that simultaneously enforces smooth constraints in homogeneous regions of the image while preserves the edges and structures without degradation. This approximation improves the existing approaches in terms of complexity of the model, as the NLM weighting function does not introduce additional parameters into the model to be estimated. Moreover, it outperforms current methods in terms of performance in a segmentation task of real world images. This work was published in the journal contribution P3 (Juan-Albarracín et al, 2019b).

\section{C3 - A method for the vascular heterogeneity assessment of glioblastoma}

The Hemodynamic Tissue Signature (HTS) method analyzes the perfusion MRI of a glioblastoma using an unsupervised learning approach to delineate four habitats within the lesion that exhibit different hemodynamic activity. The habitats describe the High Angiogenic Tumor (HAT) and Low Angiogenic Tumor (LAT) regions of the glioblastoma, and the potentially Infiltrated Peripheral Edema (IPE) and Vasogenic Peripheral Edema (VPE) of the lesion. Such approximation establishes a conceptual frame for the description of the tumor heterogeneity by means of the detection of clinically relevant sub-regions, a.k.a habitats, with differentiated imaging biomarkers. The preliminar results of this 
work were first presented in the conference contribution P4 (Juan-Albarracín et al, 2016) and it was finally published in the journal contribution P5 (JuanAlbarracín et al, 2018).

\section{C4 - Preliminary validation of the vascular heterogeneity assessment method on a local cohort of glioblastomas}

A preliminary validation study was performed to assess the association of the HTS habitats with relevant clinical outcomes. Specifically, measurements on the distributions of the hemodynamic biomarkers confined at each HTS habitat were explored for potential correlations and predictive capabilities with the overall survival of the patients. Additionally, a technical study was conducted to measure the degree of dissimilarity between these distributions, in order to confirm the physiological differences of the hemodynamic activity of the habitats. Results on a real cohort from a local hospital were published in the journal articles P5 (Juan-Albarracín et al, 2018) and P6 (Fuster-Garcia et al, 2018).

C5 - International retrospective multicenter clinical study for validating the vascular heterogeneity assessment method

The relevant findings obtained in the experiments for the preliminary validation of the aforementioned vascular heterogeneity assessment method led us to initiate an international multi-center validation study of the technology. This constituted the first clinical study in which the Universitat Politècnica de València (UPV) was sponsor. The clinical study was formally registered in the ClinicalTrial.gov platform from the U.S. National Library of Medicine with identifier NCT03439332, as seen in contribution CS. It consists of a multi-center observational retrospective study with data collected from 7 international hospitals, with a total of 305 patients enrolled since 1st of January of 2012 until February of 2018. Results obtained with this large heterogeneous cohort of untreated glioblastomas were published in the journal contribution P7 (Álvarez Torres et al, 2019), consolidating the previous findings about the predictive potential of the habitats.

\section{C6 - An online open-access system for glioblastoma MRI analysis}

This contribution consists of the development of a web-based system for the analysis of glioblastomas by means of MRI. The system, named ONCOhabitats (https://www.oncohabitats.upv.es), provides free access to all the methods developed and validated in this thesis, but also to other state-of-the-art algorithms in the field of medical image analysis, to offer a complete solution for the study of glioblastoma from raw unprocessed MRI. ONCOhabitats implements two main services to describe the morphological and vascular heterogeneity of the glioblastoma, generating for each service an automated $\mathrm{AT}_{\mathrm{E} X} \mathrm{X}$-based report summarizing all the findings of the study. The details of the system were presented in the journal contribution P8 and conference contribution P9, and the software was registered in the technological catalogue of the UPV, as shown in contributions S1 and S2.

C7 - An industrial patent for generating multi-parametric nosological images 
In addition to the scientific and academic contributions, the methods, technologies and original ideas conceived in this thesis were protected under the international patent mentioned in contribution PT. The patent issued "Method and system for generating multi-parametric nosological images" was registered in Spain (ES201431289A), with the added value of being evaluated with previous exam; was extended to the European (EP3190542A1) territory and United States (US20170287133A1) through the Patent Cooperation Treaty (PCT) programme. The patent protects a method to produce nosological images from multiple medical image acquisitions, with the aim of facilitating the diagnosis and treatment of diseases. In this sense, this thesis has contributed not only with advances in knowledge in the fields of ML and medical imaging, but with a technological asset of high value for the UPV, which opens the door to transfer actions for the creation of new business opportunities.

\section{C8 - Foundation of the ONCOANALYTICS CDX, S.L. company}

The issuance of the patent led us to participate in two of the most cutting-edge national programmes for the generation of business models and new start-ups in the field of healthcare technologies. The author of this thesis, together with the advisors, participated in the EIT Health Headstart Proof of Concept 2016 programmme, in which we were awarded the best Proof of Concept Spain for a technology-based start-up; and in the CaixaImpulse acceleration programme for facilitating entrepreneurship in biomedicine. Such mentoring activities finally led to the foundation of ONCOANALYTICS CDX, S.L. in 2018, with the commercial name Texture $C D x$, as shown in contribution TR. The company was framed into the business model of companion diagnostics for pharmaceutical compounds, with the aim of using the aforementioned vascular heterogeneity assessment technology to help in the stratification of patients affected by glioblastoma during the clinical trial of a drug. ONCOANALYTICS CDX was established by a multi-disciplinary team made up of computer scientists, physicists, oncologists, biomedical engineers and financial experts, including 6 UPV graduates and 4 Phd, thus contributing to the generation of professional opportunities for highly qualified personnel.

The work developed in this thesis has been framed in several national research projects, one of which has obtained the $\mathrm{A}+$ rating, i.e. the best rating available. This has made it possible to raise public funds, new research projects and doctoral grants that have consolidated the research line in the Biomedical Data Science Laboratory (BDSLab) of the UPV.

\subsubsection{Scientific publications}

The scientific contributions of this thesis have been published in six scientific topranked journals and five conference proceedings in the fields of Machine Learning, Statistics and Probability, Radiology \& Nuclear Medicine, Medical Imaging and Biomedical Data Mining. The publications are listed as follows: 
P1 - Javier Juan-Albarracín, Elies Fuster-Garcia, José V. Manjón, Montserrat Robles, F. Aparici-Robles, L. Martí-Bonmatí and Juan M. García-Gómez. 'Automated Glioblastoma Segmentation Based on a Multiparametric Structured Unsupervised Classification'. PLoS One; 2015; 10(5):e0125143. May 2015. (Juan-Albarracín et al, 2015b).

IF: 3.057 (JCR 2015): 11/63 Multi-disciplinary sciences (Q1).

P2 - Javier Juan-Albarracín, Elies Fuster-Garcia and Juan M. García-Gómez. 'Hierarchical Tissue-Guided Glioblastoma Segmentation based on DCA-SVFMM'. II International Symposium on Clinical and Basic Investigation in Glioblastoma. GBM2015. 3(1):101. Toledo, Spain. September 2015. (Juan-Albarracín et al, 2015a).

P3 - Javier Juan-Albarracín, Elies Fuster-Garcia and Juan M. García-Gómez. 'NonLocal Spatially Varying Finite Mixture Models for Image Segmentation'. Statistics and Computing; September 2019; Accepted for publication. (Juan-Albarracín et al, 2019b).

IF: 2.383 (JCR 2018): 16/123 Statistics \& Probability (Q1), 31/105 Computer Science, Theory \& Methods (Q2).

P4 - Javier Juan-Albarracín, Elies Fuster-Garcia and Juan M. García-Gómez. 'An online platform for the automatic reporting of multi-parametric tissue signatures: A case study in Glioblastoma'. In: Crimi A., Menze B., Maier O., Reyes M., Winzeck S., Handels H. (eds) Brainlesion: Glioma, Multiple Sclerosis, Stroke and Traumatic Brain Injuries. BrainLes 2016. Lecture Notes in Computer Science, vol 10154. Springer, Cham. Athens, Greece. October 2016. (Juan-Albarracín et al, 2016).

P5 - Javier Juan-Albarracín, Elies Fuster-Garcia, Alexandre Pérez-Girbés, F. ApariciRobles, Ángel Alberich-Bayarri, Antonio Revert-Ventura, L. Martí-Bonmatí and Juan M. García-Gómez. 'Glioblastoma: Vascular Habitats Detected at Preoperative Dynamic Susceptibility-weighted Contrast-enhanced Perfusion MR Imaging Predict Survival'. Radiology; 2018; 287(3):944-954. Jun 2018. (Juan-Albarracín et al, 2018).

IF: 7.608 (JCR 2018): 4/129 Radiology, Nuclear Medicine \& Magnetic Resonance Imaging (Q1).

P6 - Elies Fuster-Garcia, Javier Juan-Albarracín, Germán A. García-Ferrando, L. MartíBonmatí, F. Aparici-Robles and Juan M. García-Gómez. 'Improving the estimation of prognosis for glioblastoma patients by MR based hemodynamic tissue signatures'. NMR in Biomedicine; 2018; 31(12):e4006. December 2018. (Fuster-Garcia et al, 2018).

IF: 3.414 (JCR 2018): 5/41 Spectroscopy (Q1), 30/129 Radiology, Nuclear Medicine \& Magnetic Resonance Imaging (Q1), 22/73 Biophysics (Q2).

P7 - María Del Mar Álvarez-Torres and Javier Juan-Albarracín and Elies Fuster-Garcia and Fuensanta Bellvís-Bataller and David Lorente and Gaspar Reynés and Jaime Font de Mora and Fernando Aparici-Robles and Carlos Botella and Jose Muñoz-Langa and Raquel Faubel and Sabina Asensio-Cuesta and Germán A. García-Ferrando and Eduard Chelebian and Cristina Auger and Jose Pineda and Alex Rovira and Laura Oleaga and Enrique Mollà-Olmos and Antonio J. Revert and Luaba Tshibanda and Girolamo Crisi and Kyrre E. Emblem and Didier Martin and Paulina Due-Tønnessen and Torstein R. Meling and Silvano Filice and Carlos Sáez and Juan M García-Gómez. 'Robust association between vascular habitats and patient prognosis in glioblastoma: an 
international retrospective multicenter study'. Journal of Magnetic Resonance Imaging; 2019; 31(12):e4006. October 2019. (Álvarez Torres et al, 2019).

IF: 3.732 (JCR 2018): 26/129 Radiology, Nuclear Medicine \& Magnetic Resonance Imaging (Q1).

P8 - Javier Juan-Albarracín, Elies Fuster-Garcia, Germán A. García-Ferrando and Juan M. García-Gómez. 'ONCOhabitats: A system for glioblastoma heterogeneity assessment through MRI'. International Journal of Medical Informatics; 2019; 128():53-61. August 2019. (Juan-Albarracín et al, 2019a).

IF: 2.731 (JCR 2018): 57/155 Computer Science and Information Systems (Q2), 28/98 Healthcare Sciences \& Services (Q2), 11/26 Medical Informatics (Q2).

P9 - Javier Juan-Albarracín, Elies Fuster-Garcia and Juan M. García-Gómez. 'MTSimaging: multiparametric image analysis services for vascular characterization of glioblastoma'. The European Society of Magnetic Resonance in Medicine and Biology Congress. ESMRMB 2017. 30 (Suppl 1): S501-S692. Barcelona, Spain. October 2017. (Juan-Albarracín et al, 2017).

P10 - Javier Juan-Albarracín, Elies Fuster-Garcia and María del Mar Álvarez-Torres and Eduard Chelebian and Juan M. García-Gómez. 'ONCOhabitats glioma segmentation model'. In: Crimi A., Menze B., Maier O., Reyes M., Winzeck S., Handels H. (eds) Brainlesion: Glioma, Multiple Sclerosis, Stroke and Traumatic Brain Injuries. BrainLes 2019. Lecture Notes in Computer Science, vol 10154. Springer, Cham. Shenzhen, China. October 2019. (Juan-Albarracín et al, 2019c).

P11 - Juan Ortiz-Pla, Elies Fuster-Garcia, Javier Juan-Albarracín and Juan M. GarcíaGómez. 'GBM Modeling with Proliferation and Migration Phenotypes: A Proposal of Initialization for Real Cases'. In: Tsaftaris S., Gooya A., Frangi A., Prince J. (eds) Simulation and Synthesis in Medical Imaging. SASHIMI 2016. Lecture Notes in Computer Science, vol 9968. Springer, Cham. Athens, Greece. October 2016. (Ortiz-Pla et al, 2016).

\subsubsection{Software}

The research conducted in this thesis has led to the creation of ONCOhabitats platform (https://www.oncohabitats.upv.es). ONCOhabitats is an online professional system for glioblastoma analysis using MRI, which encapsulates all the original methods and algorithms developed in this thesis, and several state-of-the-art algorithms for medical image analysis. Preliminary versions of some methods of ONCOhabitats were first registered in the technological catalogue of the UPV under the acronym CURIAM $\mathrm{BT}+$, while the final updated complete version of the ONCOhabitats system has been recently registered as an asset of high value for the technological offer of the UPV.

S1 - Javier Juan-Albarracín, Elies Fuster-Garcia, Juan M. García-Gómez, Carlos Sáez, Montserrat Robles and Miguel Esparza. 'R-16874-2014 - Caracterización de firmas biológicas de glioblastomas (CURIAM BT+)'. CARTA Registry of the Universitat Politècnica de València. 28/02/2014. 
S2 - Javier Juan-Albarracín, Elies Fuster-Garcia, Juan M. García-Gómez. ' $R$ - $X X X X X$ 2019 - Detección de hábitats para evaluación de heterogeneidad biológica de glioblastomas (ONCOhabitats)'. CARTA Registry of the Universitat Politècnica de València. In process.

\subsubsection{Clinical studies}

The aforementioned ONCOhabitats platform is currently enrolled in an international multicenter observational restrospective clinical study registered at ClinicalTrials.gov from the U.S. National Library of Medicine. The aim of the study is to validate the prognostic capabilities of the HTS habitats for patients affected with glioblastoma. To this end, the primary and secondary outcomes fixed for the clinical study are: the "correlation between overall survival and progression-free survival (in days) of patients undergoing standard-of-care treatment and the tumor vascular heterogeneity described by the four habitats obtained by the HTS biomarker". The clinical study involves data collected from 7 international hospitals, with a total of 305 patients recruited since 1st of January of 2012 until February of 2018.

CS - Multicenter Retrospective Observational Clinical Study NCT03439332. 'Multicentre Validation of How Vascular Biomarkers From Tumor Can Predict the Survival of the Patient With Glioblastoma (ONCOhabitats)'. https://clinicaltrials.gov/ct2/show/ NCT03439332. Universitat Politècnica de València (UPV). 20/02/2018.

\subsubsection{Patents}

The know-how in medical image analysis generated by the author in this thesis was protected under the international patent "Method and system for generating multiparametric nosological images". The patent is currently registered in Spain (ES201431289A) with previous exam, and was extended to Europe (EP3190542A1) and United States (US20170287133A1) following the PCT procedure for patent internationalization. It describes a procedure based on multi-parametric medical images to generate nosological masks capable of describing the underlying physiological processes taking place in the lesion. The patent materializes the interest that the scientific research conducted in this thesis has originated in both the academic and business spheres. Moreover, it represents the efforts of this thesis to generate tangible assets for a later phase of business development.

PT - Javier Juan-Albarracín, Elies Fuster-Garcia, Juan M. García-Gómez, Miguel EsparzaManzano, Jose V. Manjón-Herrra, Monserrat Robles-Viejo, Carlos Sáez. 'Method and system for generating multi-parametric nosological images'. Asignee: Universitat Politècnica de València (UPV).

ES201431289A: Oficina Española de Patentes y Marcas. 05/09/2014. Legal status: Active.

EP3190542A4: European Patent Office. PCT/ES2015/070584. 28/07/2015. Legal status: Active.

US9990719B2: United States Patent and Trademark Office. PCT/ES2015/070584. 28/07/2015. Legal status: Active. 


\subsubsection{Transference}

The experience, knowledge and original ideas conceived in this thesis, together with the issuance of the patent, aroused the author's interest in taking a step beyond the academic field. This led the author and the thesis advisors to the conceptualization of a business plan to capitalize the results obtained in the thesis. In this sense, we participated in the EIT Health Headstart Proof of Concept 2016 programmme, in which we were awarded the best business plan for a biotech start-up; and in the CaixaImpulse programme for facilitating entrepreneurship in biomedicine. The experience obtained in these programmes in conjunction with the background of the advisors in generating spin-offs of the UPV, finally led to the foundation of ONCOANALYTICS CDX company. ONCOANALYTICS CDX is formed by a multi-disciplinary team made up of computer scientists, physicists, oncologists, biomedical engineers and financial experts, with a total of 6 UPV graduates and 4 Phd. Supported by the aforementioned ONCOhabitats platform, the company is focused in developing image-based CDx for glioblastoma, to facilitate patient stratification during the clinical trial of a drug.

PT - Javier Juan-Albarracín, Elies Fuster-Garcia, Juan M. García-Gómez, Germán A. García-Ferrando, Carlos Vidal-Trujillo, José Muñoz-Langa, David Lorente-Estellés, Ana González-Segura, Fuensanta Bellvís-Bataller. 'ONCOANALYTICS CDX S.L.'. Commercial name: Texture CDx. CIF: B98981889. 01/03/2018.

\section{$1.4 \quad$ Projects and partners}

During the development of this thesis the author has actively participated in several national, European and private research projects in collaboration with several hospitals and clinical institutions. The projects related to this thesis are listed below:

CURIAM BT + Caracterización de firmas biológicas de glioblastomas mediante modelos no-supervisados de predicción estructurada basados en biomarcadores de imagen. Funded by the Spanish Ministry of Economy and Competitiveness (TIN201343457-R, 2014-2016).

Objectives: This project aims to develop a computational medical imaging system to obtain radiological profiles of the different areas of the tumor, to accurately measure the vascular properties of the glioblastoma. Such profiles will also provide information about the tumor grading and the expected survival of the patient.

Partners: Biomedical Data Science Laboratory (BDSLab)-ITACA group of the Universitat Politècnica de Valencia (Valencia, Spain). Hospital Universitario y Politécnico La Fe (HUPLF) (Valencia, Spain).

MTS4Up Biomarcadores dinámicos basados en firmas tisulares multiparamétricas para el seguimiento y evaluación de la respuesta a tratamiento de pacientes con glioblastoma y cáncer de próstata. Funded by the Spanish National Research Agency (DPI201680054-R, 2017-2018). 
Objectives: This project extends the TIN2013-43457-R project by improving the technology to obtain radiological signatures of the glioblastoma incorporating diffusion MRI to describe not only tumor vascularity, but also the cell density properties of the tissues. Such improvements will allow an early evaluation of tumor progression and an accurate assessment of the patient's response to treatment. Finally, the technology will also be evaluated on other pathologies such as prostate tumor to measure the versatility of the methodology in other solid tumors.

Partners: Biomedical Data Science Laboratory (BDSLab)-ITACA group of the Universitat Politècnica de Valencia (Valencia, Spain).

GLIO-MARKERS Estudio integrado de biomarcadores moleculares y de imagen en pacientes con glioblastoma. Funded by the Universitat Politècnica de València and Hospital Universitario y Politécnico La Fe (Prueba de Concepto 2015, UPV-FE-15-B, 2015-2016).

Objectives: This project aims to combine and integrate the analysis of glioblastoma biomarkers from three different physiological areas: blood circulating proteins, immunohistologic biomarkers and MRI biomarkers. The purpose is to develop predictive models for response to treatment assessment and measuring tumor progression, as well as finding correlations between imaging biomarkers and circulating proteins.

Partners: Biomedical Data Science Laboratory (BDSLab)-ITACA group of the Universitat Politècnica de Valencia (Valencia, Spain), Universitat Politècnica de València (UPV) (Valencia, Spain).

DSSRADIOPLAN Inclusión de las tecnologías de firma tisular y modelos mutiescala para el soporte a la planificación de la radioterapia en el tratamiento del glioblastoma. Funded by the Universitat Politècnica de València and Hospital Universitario y Politécnico La Fe (Prueba de Concepto 2016, UPV-FE-16-B, 2016-2017).

Objectives: The main purpose of this project is to plan and carry out the necessary actions to evaluate the applicability and added value of the HTS technology to provide clinical decision support in the management and planning of radiotherapy in patients affected by glioblastoma.

Partners: Biomedical Data Science Laboratory (BDSLab)-ITACA group of the Universitat Politècnica de Valencia (Valencia, Spain), Universitat Politècnica de València (UPV) (Valencia, Spain).

MULTIBIOIM Multiparametric nosological images for supporting clinical decisions in solid tumors. Funded by the EIT Health E.V. (Proof of Concept 2016, POC-2016SPAIN-07, 2016-2017).

Objectives: This project aims to develop a Proof of Concept (PoC) of the patented procedure ES201431289A for generation of multiparametric tissue signatures based on structural and functional MRI for solid tumors. This PoC aims to solve specific technical, strategical, legal, and commercial barriers to generate a reliable business model 
for the medical image analysis software market and convert a cutting-edge technology into a clinically validable product.

Partners: BDSLab-ITACA group of the Universitat Politècnica de Valencia (Valencia, Spain).

The projects on which the author was involved in previously and in parallel to the development of this thesis are listed as follows:

DQV-MINECO Servicio de evaluación y rating de la calidad de repositorios de datos biomédicos. Funded by the Spanish Ministry of Economy and Competitiveness (Retos-Colaboración 2013 programme, RTC-2014-1530-1, 2013-2016).

Objectives: This project aims to define a data quality evaluation and rating service to assure the data value aimed to its reuse in clinical, strategic and scientific decision making. It will be based on two software services. The first will evaluate nine data quality dimensions. The second will generate a data quality rating positioning the evaluated datasets according to several reuse knowledge extraction purposes.

Partners: VeraTech for Health S.L. (Valencia, Spain) and IBIME-ITACA group of the Universitat Politècnica de Valencia, (Spain)

HELP4MOOD A Computational Distributed System to Support the Treatment of Patients with Major Depression. Funded by the European Commission. VII Framework Program (FP7-ICT-2009-4; 248765, 2011-2013).

Objectives: This project focuses on major depression disease. Patients with major depression typically recover through antidepressant drugs, psychological therapy or hospitalization. However, it has been shown that in many situations such recovery is either slow or incomplete. Research shows that psychological therapies can be delivered effectively without face to face contact at individual's home by computerized cognitive behavioral therapy. The project aims to advance the state-of-the-art in computerized support for people with major depression by monitoring mood, thoughts, physical activity and voice characteristics, by means of intelligent systems based on virtual agent.

Partners: BDSLab-ITACA group of the Universitat Politècnica de Valencia, (Spain), University of Edinburgh (United Kingdom), Fundació I2CAT (Spain), Universitatea Babes Bolyai (Romania), FVA SAS di Louis Ferrini (Italy), OBS Medical Ltd. (Italy), Universitat Politècnica de Catalunya, (Spain), Heriot-Watt University (United Kingdom).

\subsection{Thesis outline}

The thesis is structured in eight chapters that thoroughly describe the research work carried out during the thesis. The Chapter 1 has introduced the motivations, research objectives and main contributions. Chapter 2 describes the thesis rationale, introducing the clinical problems addressed as well as the theoretical background needed to 
complement the description of the methods developed in the thesis. Chapter 3 presents a preliminary study on the viability of the unsupervised learning paradigm to identify and delineate pathological tissues in glioblastomas based on MRI patterns. Chapter 4 presents the mathematical development of a new unsupervised structured learning algorithm for image segmentation, and a comparison of its performance against alternative approaches. Chapter 5 introduces the HTS method: an unsupervised learning method based on perfusion MRI to delineate vascular habitats within the glioblastoma to assess its vascular heterogeneity. An study on the association of the vascular habitats and the patient OS is presented. Chapter 6 describes the international multi-center validation of the HTS method, under the framework of the observational retrospective clinical study NCT03439332. The validation of the association of the vascular habitats and the patient OS, as well as the stratification capabilities of the HTS habitats is presented. Chapter 7 presents ONCOhabitats platform (https://www.oncohabitats.upv.es). ONCOhabitats encapsulates all the work conducted in this thesis in a public open-access platform, offering medical image analysis services to analyze both the morphological and vascular heterogeneity of the glioblastoma. Finally, chapter 8 ends this dissertation with the concluding remarks and recommendations to continue with the research developed in this thesis.

Figure 1.1 outlines the thesis contributions structured among the thesis chapters, along with the publications, research projects, transfer actions, patents and the software developed during this study. 


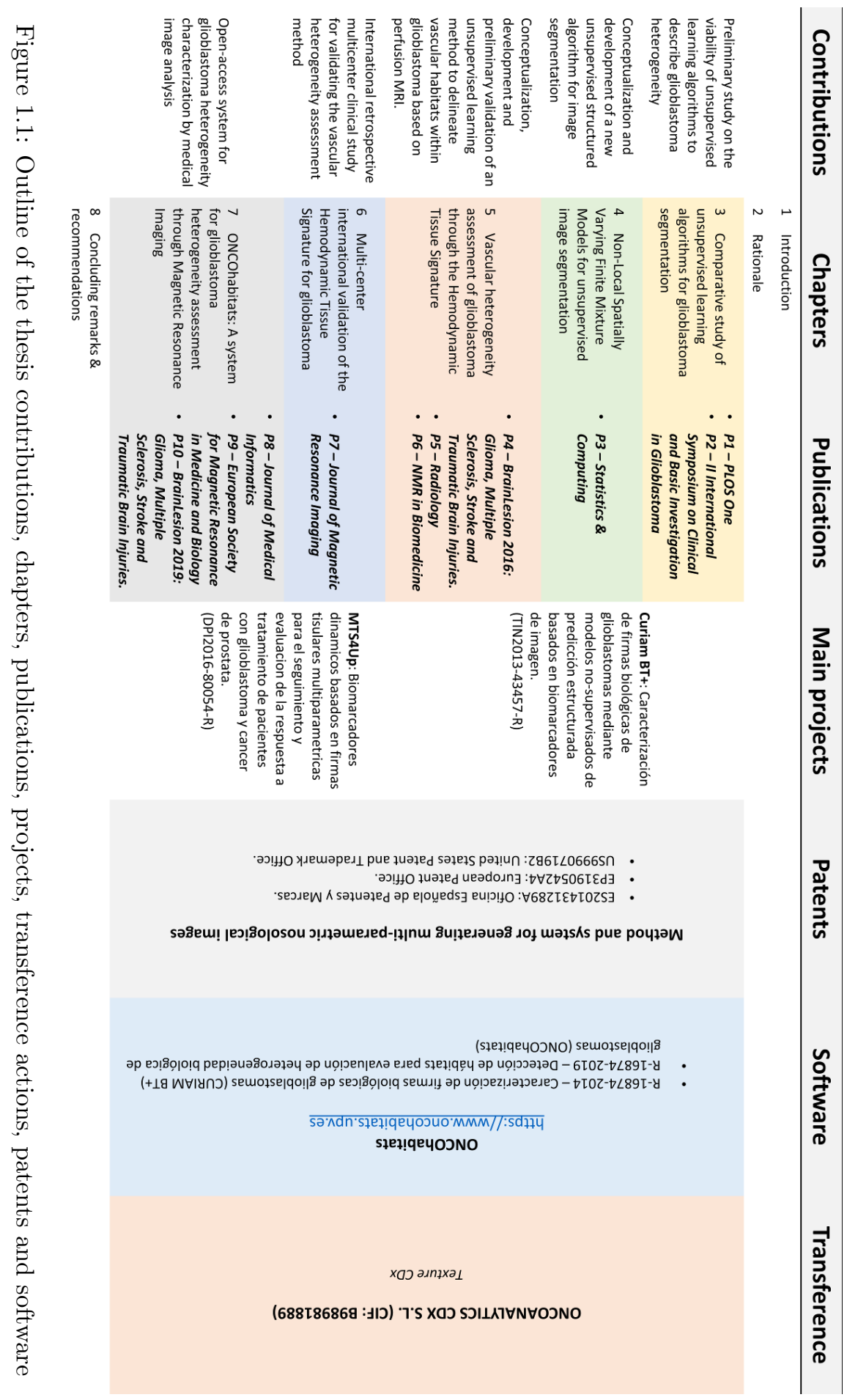


Chapter 1. Introduction 


\section{Chapter 2}

\section{Rationale}

This chapter describes the thesis rationale divided in five sections. First, the glioblastoma tumor is introduced, describing its epidemiology, etiology, biologic behavior, morphological features, diagnosis and treatment. Second, MRI technique is disclosed, illustrating their physical mechanisms, theoretical foundations and acquisition protocols and sequences. Third a general review on the theoretical background probability and statistical parameters estimation recommended for the understanding of the methods developed in the thesis is provided. Fourth, an in-depth explanation of Finite Mixture Model and Spatially Varying Finite Mixture Model and their parameter estimation is presented to lay the foundation for many of the methods developed in this thesis. Finally, a general review of the DL paradigm, as well as a revision of Artificial Neural Networks, Convolutional Neural Networks and the back-propagation mechanics is provided. This review is intended to establish a common basis to complement the descriptions of background and methods explained in the following chapters.

\subsection{Glioblastoma}

The first recorded clinical report identifying glioblastoma as a tumor originating from neuroglial cells dates back to 1863 by Virchow (2018). Since then, enormous progress has been made in the understanding of this neoplasm thanks to an exhaustive multidisciplinary research in the clinical, pathological, radiological, molecular and genetic aspects of the tumor. Such efforts have led nowadays to a detailed description of the glioblastoma that has given us crucial, but still not sufficient, information to design successful treatments for this disease.

Glioblastoma is a grade IV World Health Organization (WHO) deadly primary brain tumor considered the most aggressive neoplasm of the central nervous system. It is the most frequent and malignant astrocytoma in humans, accounting for more than $60 \%$ of all brain tumors in adults. Glioblastoma has a global incidence of 4.67 to 5.73 per 100000 people and presents a poor prognosis of 14-15 months despite aggressive treatments. Although it can debute at any age, more than the $70 \%$ of the cases are seen in patients between the ages of 45 and 70 . Likewise, the incidence in males is 1.6 higher than in females and it is 2 times higher in Caucasians than in other races (Tamimi and Juweid, 2017). 
Glioblastomas are infiltrative and deeply invasive heterogeneous masses characterized by hypercellularity, pleomorphism, microvascular proliferation and high necrosis mitotic activity (Gladson et al, 2010). Typically, glioblastoma exhibits diffuse margins with co-existence of different tissues including active tumor, cysts, necrosis and edema; all of them exhibiting a high variability related to the aggressiveness of the neoplasm (Hardee and Zagzag, 2012). Strong vascular proliferation, robust angiogenesis, and extensive microvasculature heterogeneity are major pathological hallmarks that differentiate glioblastomas from low-grade gliomas (Kargiotis et al, 2006).

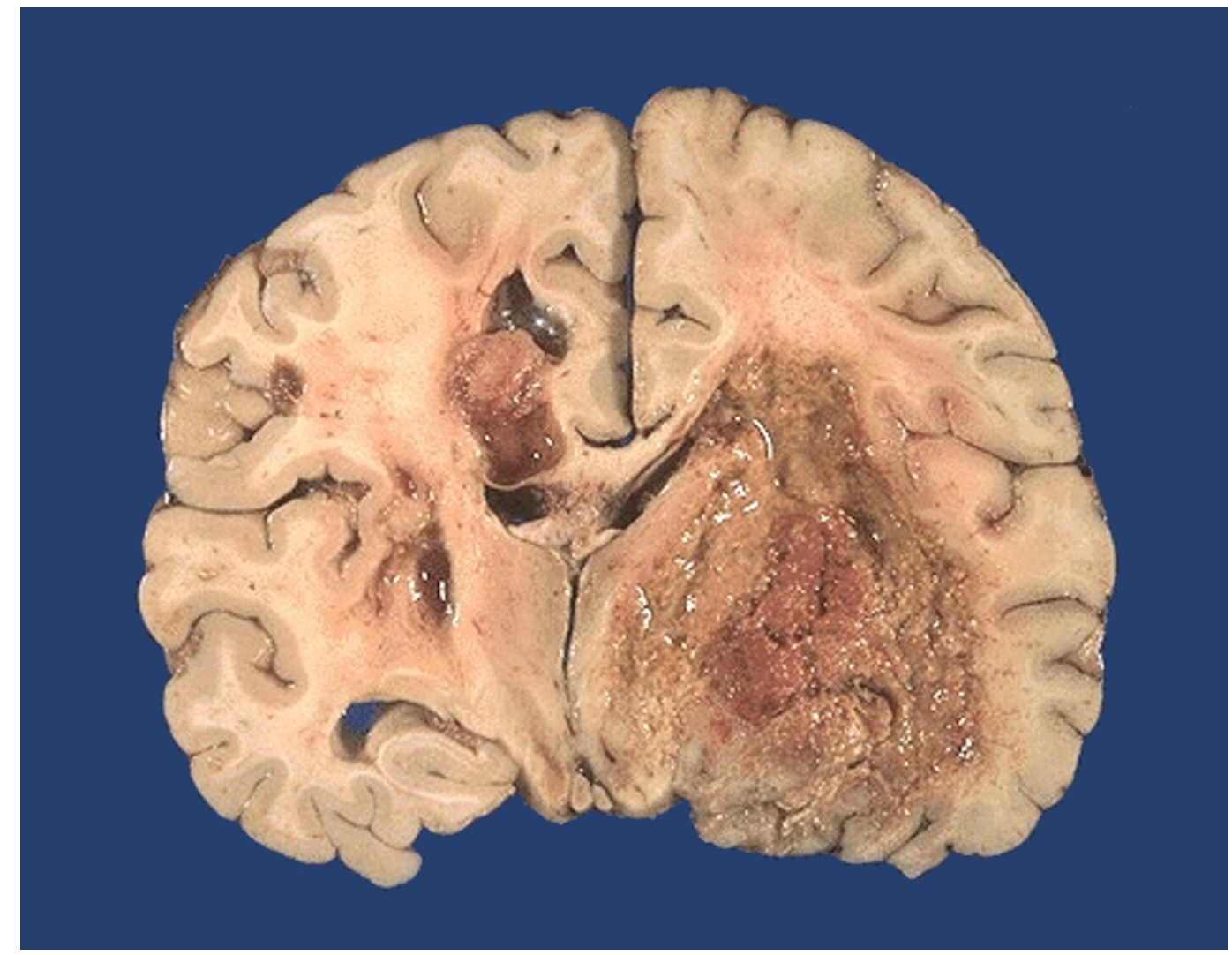

Figure 2.1: Macroscopic example of glioblastoma. Image taken with kind permission from https: //webpath.med.utah.edu/CNSHTML/CNS136.html

Figure 2.1 shows an example of a brain affected by glioblastoma. Note the deep invasive ability of the tumor, evidenced by the tumor foci crossing the midline towards the opposite hemisphere of the largest mass.

Heterogeneity has therefore been considered crucial to understand the aggressiveness of this tumor and its resistance to effective therapies. Glioblastoma heterogeneity manifests itself at both macroscopic and microscopic levels. At the macroscopic level, co-existance of an amalgam of blended malignant tissues including enhancing tumor, non-enhancing tumor, hemorrhage, cyst, inflammation, necrosis or edema, results in a chaotic mass highly complicated to manage clinically. At the microscopic level, different glioblastoma molecular sub-types and genetic alterations have been discovered in the past years. In 2010, Verhaak et al (2010) established a classification for glioblastoma into four sub-types associated to mutations in EGFR, TP53, NF1 and 
PDGFRA/IDH1 genes: the classical, mesenchymal, neural and proneural sub-types. The main characteristics of these tumor sub-types are summarized in Table 2.1.

Table 2.1: Summary of the most relevant characteristics of Verhaak glioblastoma subtypes classification. ${ }^{*}$ LOH: Loss of heterozygosity.

\begin{tabular}{|c|c|}
\hline CLASSICAL & MESENCHYMAL \\
\hline $\begin{array}{l}\text { - High EGFR (97\%) } \\
\text { - Lack of TP53 mutations } \\
\text { - Chromosome } 7 \text { amplification with } \\
\text { LOH* chromosome } 10 \\
\text { - CDKNA2 deletion }(94 \%) \\
\text { - High Notch and Sonic Hedgehog } \\
\text { markers } \\
\text { - Patients survive longest given ag- } \\
\text { gressive treatment }\end{array}$ & $\begin{array}{l}\text { - Focal deletions at 17q11.2 } \\
\text { - Mutated NF1 (70\%) } \\
\text { - Mutated TP53 } \\
\text { - Mutated PTEN } \\
\text { - Expression of CH13L1 marker } \\
\text { - Expression of MET marker } \\
\text { - Higher activity of astrocytic } \\
\text { markers (CD44 and MERTK) } \\
\text { - Increased NF-kB pathway }\end{array}$ \\
\hline PRONEURAL & NEURAL \\
\hline $\begin{array}{l}\text { - Altered PDGFRA } \\
\text { - Point mutations at IDH1 }(93 \%) \\
\text { - TP53 LOH }(67 \%) \\
\text { - Lesser chromosome } 7 \text { amplifica- } \\
\text { tion with LOH chromosome } 10 \\
\text { - Focal amplifications at } 4 q 12 \\
\text { (higher than other subtypes) } \\
\text { - Expression of oligodendrotytics } \\
\text { genes }\end{array}$ & $\begin{array}{l}\text { - Neuron markers (NEFL, } \\
\text { GABRA1, SYT1, SLC12A5) } \\
\text { - Few }(75 \%) \text { has normal cells in } \\
\text { pathology slides } \\
\text { - Association with oligodendrocytic } \\
\text { and astrocytic differentiation but } \\
\text { mostly express neuron markers }\end{array}$ \\
\hline
\end{tabular}

In 2016, the WHO revisited the official classification for glioblastoma sub-types, distinguishing between two groups: the IDH-wildtype (90\% of cases) and the IDH-mutant (10\% of cases), which are closely related to primary and secondary glioblastomas respectively. Table 2.2 summarizes the most relevant aspects of the IDH-wildtype and IDH-mutant glioblastomas.

The study of these transcriptional subtypes has yielded relevant findings such as significant correlation with patient prognosis (Parsons et al, 2008). IDH-mutant glioblastomas show a significant improvement in OS with a median survival of 31 months, with respect to IDH-wildtype glioblastomas that present a median survival of 15 months 
Table 2.2: Summary of the most relevant aspects of IDH-wildtype and IDH-mutant glioblastomas.

\begin{tabular}{lll}
\hline & IDH-wildtype glioblastomas & IDH-mutant glioblastomas \\
\hline Synonym & Primary glioblastoma & Secondary glioblastoma \\
Precursor lesion & Not identifiable; develops & Diffuse astrocytoma, \\
& de novo & Anaplastic astrocytoma \\
Proportion of glioblastomas & $\sim 90 \%$ & $\sim 10 \%$ \\
Median age at diagnosis & $\sim 62$ years & $\sim 44$ years \\
Male-to-female ratio & $1.42: 1$ & $1.05: 1$ \\
Mean length of clinical history & 4 months & 15 months \\
Median overall survival & & \\
$\quad$ Surgery + radiotherapy & 9.9 months & 24 months \\
$\quad$ Surgery + radiotherapy & 15 months & 31 months \\
Location & Supratentorial & Preferentially frontal \\
Necrosis & Extensive & Limited \\
TERT promoter status & $72 \%$ & $26 \%$ \\
TP53 mutations & $27 \%$ & $81 \%$ \\
ATRX mutations & Exceptional & $71 \%$ \\
EGFR mutations & $35 \%$ & Exceptional \\
PTEN mutations & $24 \%$ & Exceptional \\
\hline
\end{tabular}


Louis et al (2016). However, although tumor subtypes tend to correlate with relevant clinical outcomes, the degree of correlation is often moderate and contradictory studies constantly appear with confronted conclusions (Akagi et al, 2018). Moreover, survival rates have shown no notable improvement since the last three decades (Stupp et al, 2005), so alternative approaches are required to study the glioblastoma heterogeneity and its association with the tumor evolution.

In this sense, significant interest has been placed recently in the analysis of glioblastoma heterogeneity through medical imaging. The ability to discover non-invasive markers associated with tumor sub-types, OS, Progression Free Survival (PFS) or response to treatment has received much attention as it may help in improving clinical decision making at an early stage of the disease. In this sense, MRI emerged as one of the most reliable tool for quantifying in-vivo non-invasive imaging features capable to accurately describe the heterogeneity of the glioblastoma.

\subsection{Magnetic Resonance Imaging}

Magnetic Resonance Imaging (MRI) is a medical imaging technique used to provide invivo internal representations of the human body. This technique was developed in the decade of 1970 by the professors Damadian (1971); Lauterbur (1973, 1974); Mansfield (1977). Although Damadian's work on the Nuclear Magnetic Resonance (NMR) relaxation of different tissues laid the groundwork for many further developments in MRI, it was Paul Lauterbur who finally developed a reliable technique based on gradient magnets to generate the first 2D and 3D Magnetic Resonance (MR) images of the interior of the human body. A few years later Peter Mansfield developed a mathematical formulation that dramatically accelerated the acquisition of MR images (seconds rather than hours), making it a practical technique for the clinical routine. Paul Lauterbur and Peter Mansfield finally awarded the Nobel prize in 2003 for their contributions and advances in MRI.

MRI is based on the magnetic properties of the atomic nuclei, specifically on the spin angular momentum of the hydrogen nucleus $\left(\mathrm{H}^{+}\right)$. At a resting natural state, all the hydrogen $H^{+}$nucleus in the human body spin randomly, thus canceling the angular momentum each other and producing an overall zero spin magnetic momentum. Under the influence of an external uniform magnetic field $B_{0}$, the $H^{+}$nuclei align their spin with $B_{0}$ in a parallel (low energy) or anti-parallel (high energy) state, producing an overall spin magnetic momentum $M_{z}$, with $z$ the direction of $B_{0}$ (see figure 2.2). The influence of $B_{0}$ also makes the $H^{+}$nuclei to precess at a specific frequency (denominated the Larmor frequency), which depends on the strength of $B_{0}$ and the gyromagnetic properties of the hydrogen nucleus.

The NMR phenomena is related to the excitation produced to the $H^{+}$nuclei by the influence of an additional temporal magnetic field (radio-frequency pulse) with a direction different from $B_{0}$. When a radio-frequency pulse at the Larmor frequency is triggered at $B_{0}$, an energy exchange occurs with some $H^{+}$nuclei, lifting them to the high energy anti-parallel state. In addition, a synchronization of the precession of all the $H^{+}$nuclei is induced, so that they all begin to precess in phase. This event causes two magnetic effects in the system: 1) a decrease in the overall spin magnetic 


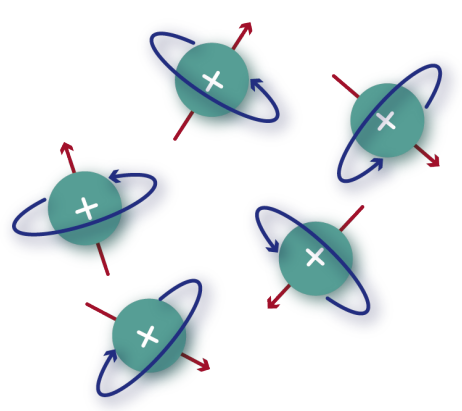

No Field

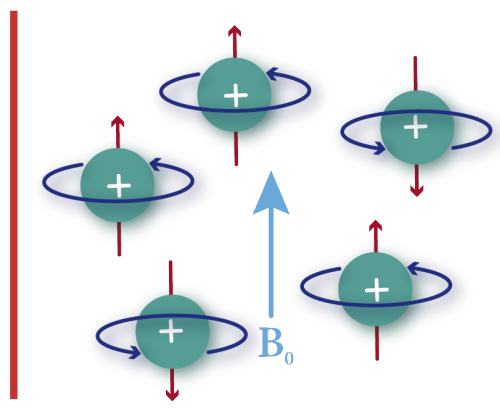

With Field

Figure 2.2: Diagram of the spin angular momentum of $H^{+}$nuclei at a resting natural state (left) and under the influence of an uniform magnetic field $B_{0}$ (right).

momentum $M_{z}$ due to the lifted $H^{+}$nuclei; and 2) the apparition of a spin magnetic momentum $M_{x y}$ transverse to the $B_{0}$ field due to the phase coherence precession. Such transverse magnetization $M_{x y}$ generates a magnetic signal called the Nuclear Magnetic Resonance Signal. Figure 2.3 shows a diagram of the effect produced by the radiofrequency pulse on the spin angular momentum of the $H^{+}$nuclei.
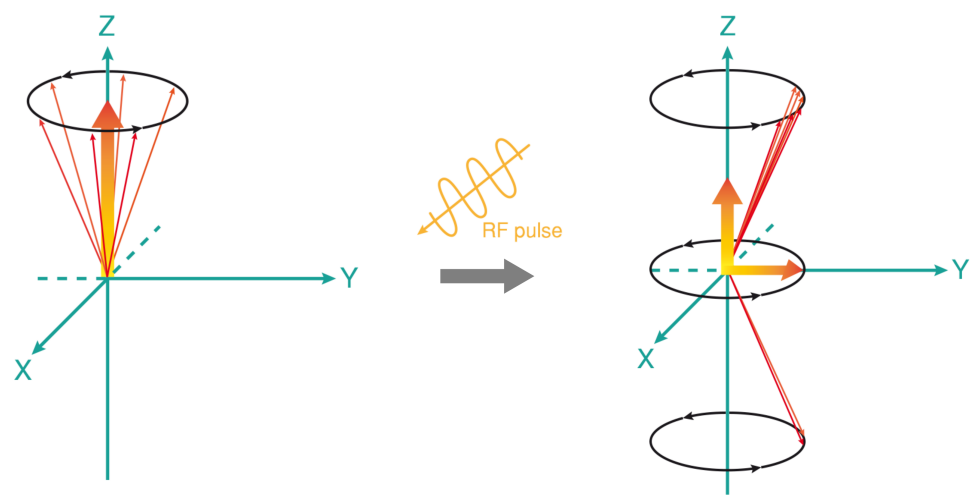

Figure 2.3: Diagram of the effect produced by the radio-frequency pulse on the spin angular momentum of the $H^{+}$nuclei. Some $H^{+}$nuclei lift their state to the anti-parallel high energy mode and a precession synchronization is attained.

Once the radio-frequency pulse has ended, the system begins to relax, recovering its initial state of equilibrium. The precession of the $H^{+}$nuclei begins to lose the phase coherence due to the differences in the chemical context of each $\mathrm{H}^{+}$nucleus. Therefore, the transverse spin magnetic momentum $M_{x y}$ begins to disappear, leading to the so-called spin-spin relaxation or transverse $\mathrm{T}_{2}$ relaxation process. Likewise, the previously lifted $\mathrm{H}^{+}$nuclei return to their original low energy parallel state, restoring the longitudinal magnetization $M_{z}$ to its original value. This process is called the spin-lattice relaxation or longitudinal $\mathrm{T}_{1}$ relaxation (see figure 2.4). 


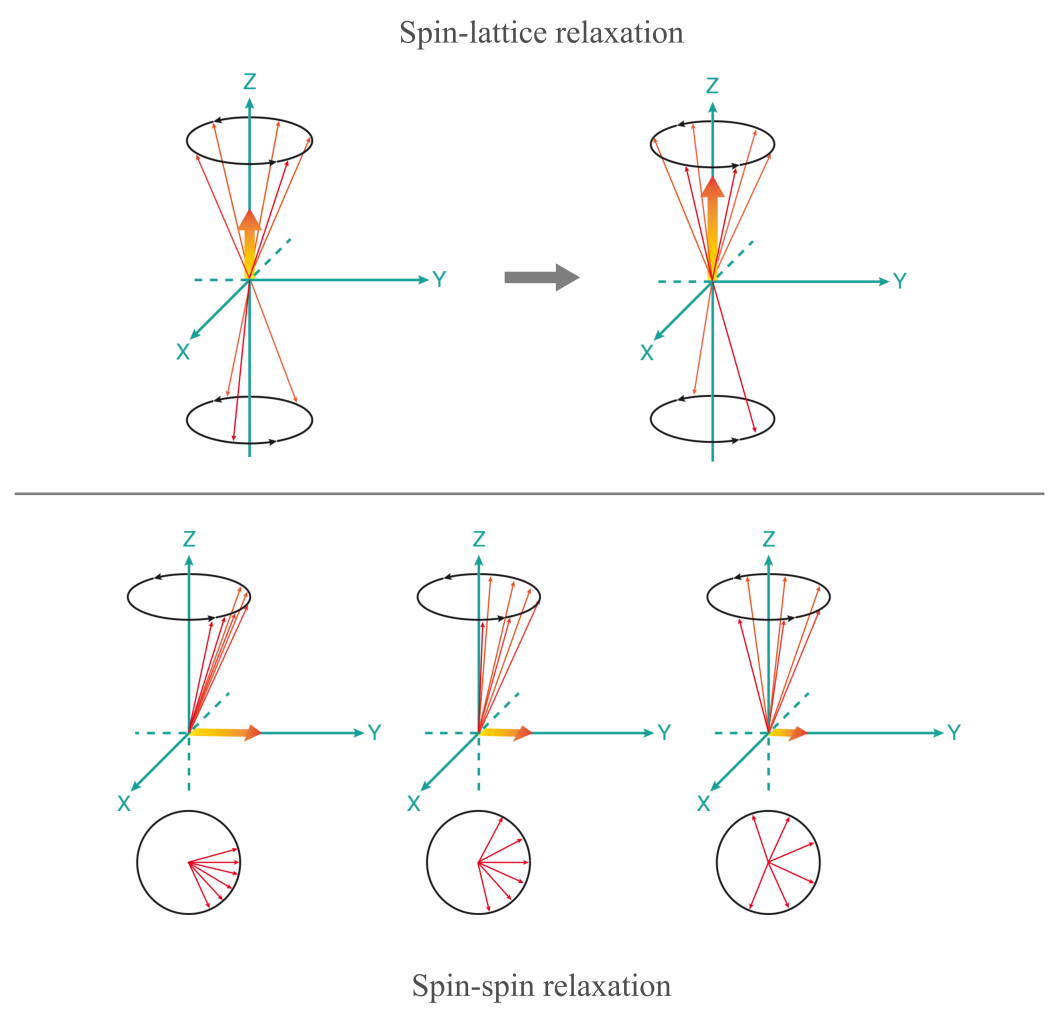

Figure 2.4: Diagram of the relaxation process after the radio-frequency pulse ends. On top, the $\mathrm{T}_{1}$ spin-lattice relaxation, showing a recovery of the original $M_{z}$ magnetization. On bottom, the $\mathrm{T}_{2}$ spin-spin relaxation, demonstrating the lose of the $M_{x y}$ magnetization due to the phase de-coherence.

$\mathrm{T}_{1}$ and $\mathrm{T}_{2}$ relaxation times are significantly different between them, with $\mathrm{T}_{1}$ a longer process than $\mathrm{T}_{2}$. Generally, $\mathrm{T}_{1}$ relaxation time ranges from 300 to $2000 \mathrm{~ms}$ while $\mathrm{T}_{2}$ relaxation is about 30 to $150 \mathrm{~ms}$. Moreover, it is difficult to determine the end of the $T_{1}$ and $T_{2}$ relaxations exactly. Therefore, it is considered that the $T_{1}$ relaxation is completed once the signal recovers the $63 \%$ of the original $M_{z}$ magnetization. Similarly, the $\mathrm{T}_{2}$ relaxation is considered ended once the $M_{x y}$ signal falls under the $37 \%$ of its original value.

As stated above, the $\mathrm{T}_{1}$ and $\mathrm{T}_{2}$ relaxation is different for each $H^{+}$nucleus depending on its chemical context. Hence, different NMR signals are emitted during the relaxation process, inducing dissimilar electric signals in the receiver coils of the MR machine. Such disparities will in essence determine the intensities for each tissue in the MR image. There are three different types of contrast in MR images: $\mathrm{T}_{1}$-weighted, $\mathrm{T}_{2}$-weighted and Proton Density (PD) images; which are related to the so-called Repetition Time (TR) and the Echo Time (TE) times. TR is the time between successive radio-frequency pulses and affects the speed in which $H^{+}$nuclei realigns to the $B_{0}$ field. The TE refers to the time at which the electrical signal induced by the $H^{+}$nuclei is measured in the magnetic coils and concerns the degree of dephase of the spins of the $H^{+}$nuclei. Thus, the TR is closely connected to $\mathrm{T}_{1}$ relaxation effects, while TE is more related to $\mathrm{T}_{2}$ relaxation events. 
Figure 2.5 shows the relation between the TR, TE and the contrast produced on the MR images.

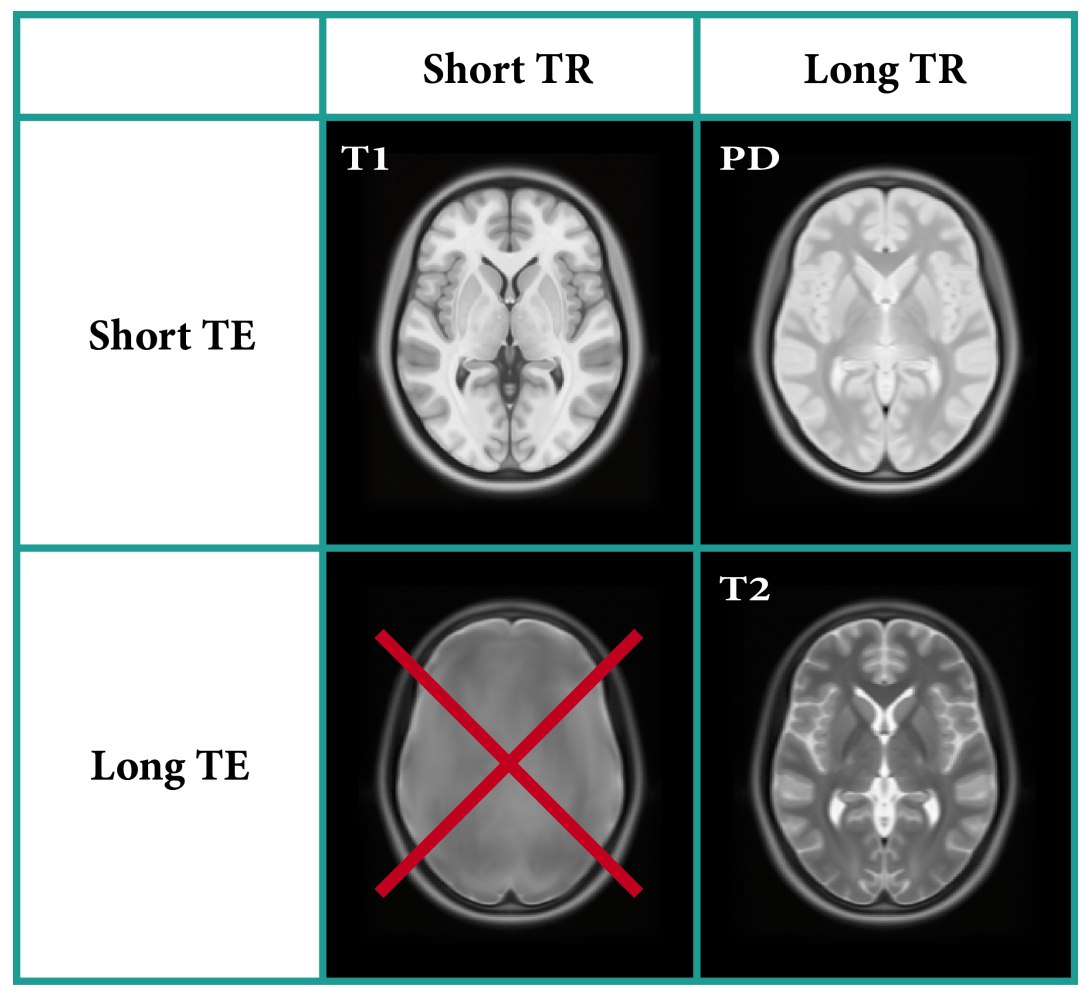

Figure 2.5: Relation between the TR and TE and the intensity contrast produced in the MR images.

\subsubsection{Anatomical MRI}

So far we have seen that NMR relaxation process is different for each tissue depending on its chemical context. This property allows to visually differentiate human tissues via the measurement of their associated electric signals, generating images that depict the anatomical structures of the region under study.

In the case of central nervous system tumors such as glioblastoma, several images are typically acquired to best approach the lesion. These images intend to anatomically describe the morphology of the lesion and its associated structures.

A common protocol for a MRI glioblastoma study should include the following sequences:

$\mathbf{T}_{1}$-weighted : typically a volumetric 3D Fast Spin Echo (FSE) or MagnetizationPrepared Rapid Acquisition with Gradient Echo (MPRAGE) sequence. The aim of the sequence is the anatomical overview of the lesion, including the soft tissues below the base of skull.

$\mathbf{T}_{1 C E}$-weighted : typically a post-contrast volumetric 3D FSE or MPRAGE sequence. It is a $\mathrm{T}_{1}$-weighted image acquired with administration of Gadolinium-Based Contrast Agent (GBCA). Its main purpose is to assess vascular structures of 
the region under study. The list of approved contrast-agents for human use is: gadoterate meglumine (Dotarem $\left.{ }^{\circledR}\right)$, gadobutrol (Gadavist ${ }^{\circledR}$ ), gadopentetate dimeglumine (Magnevist ${ }^{\circledR}$ ), gadobenate dimeglumine (MultiHance ${ }^{\circledR}$ ), gadodiamide (Omniscan ${ }^{\circledR}$ ), gadoversetamide (OptiMARK ${ }^{\circledR}$ ), gadoteridol (ProHance ${ }^{\circledR}$ ).

$\mathbf{T}_{2}$-weighted : typically a $2 \mathrm{D}$ axial FSE sequence. Its purpose is the evaluation of basal cisterns, ventricular system and subdural spaces, evaluation of vasogenic edema and good visualization of flow-voids in vessels.

FLAIR : typically a $2 \mathrm{D}$ axial Fluid Attenuation Inversion Recovery (FLAIR) $\mathrm{T}_{2^{-}}$ weighted sequence. It is a special inversion recovery sequence with a long inversion time. Its main characteristic is that it suppress the signal from the cerebrospinal fluid so that it appears similar to a $\mathrm{T}_{2}$-weighted image but with the cerebrospinal fluid dark instead of bright. Its purpose is the assessment of white-matter tumor involvement and related vasogenic edema.

Figure 2.6 shows an example of the aforementioned MR images for a case affected by glioblastoma.
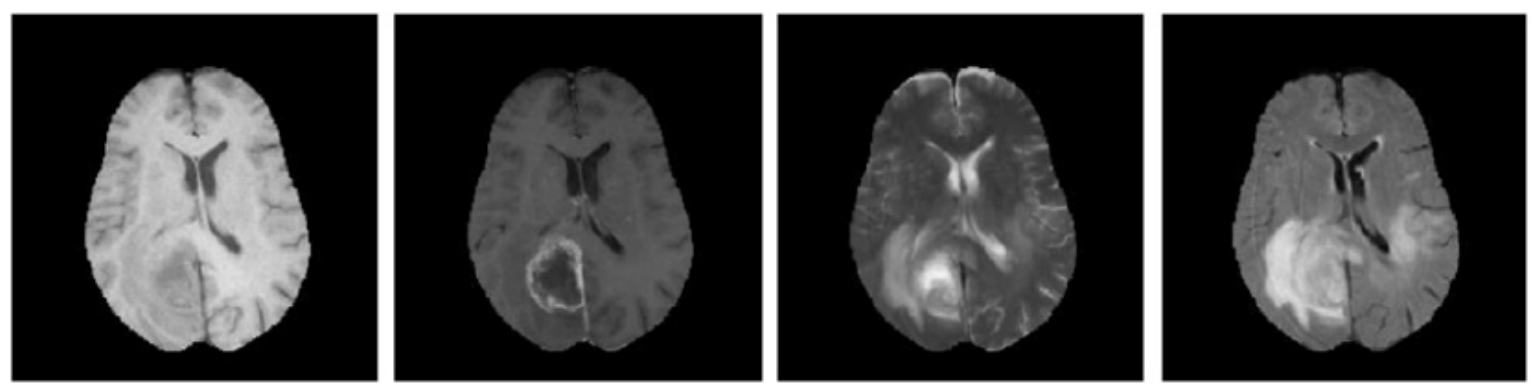

Figure 2.6: Example of anatomical MR images typically acquired in a glioblastoma MR study. From

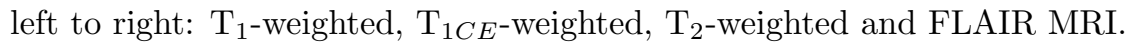

\subsubsection{Quantitative MRI}

Quantitative Magnetic Resonance Imaging (qMRI) emerged in the 1980's in the attempt to measure the biological MR properties of the tissues (Tofts, 2004). Therefore, its purpose is to turn the MRI into a scientific measurement instrument and not only on an image acquisition system. This concept-shift is the essence of qMRI. qMRI attempts to develop quantitative and reproducible techniques based on MR data, able to measure biomarkers related to the underlying biological processes of the tissues (Yankeelov et al, 2011). MRI Relaxometry (Deoni, 2010), Diffusion Weighted Imaging (DWI) (Schaefer et al, 2000) and Perfusion Weighted Imaging (PWI) (Svolos et al, 2014) composes some of the most important techniques of qMRI.

Regarding glioblastoma tumors, one of the most relevant qMRI techniques is PWI. As previously mentioned, glioblastoma tumors are characterized by a strong vascular proliferation, robust angiogenesis, and extensive microvasculature heterogeneity. In this sense, PWI allows the measurement of the kinetic properties of a paramagnetic contrast agent, which is intravenously injected to the patient. PWI biomarkers are able 
to reveal the local vascular properties of the tissues and their hemodynamic behavior. There are three main PWI techniques: Dynamic Susceptibility Contrast (DSC) perfusion, Dynamic Contrast Enhanced (DCE) perfusion and Arterial Spin Labeling (ASL) perfusion. For reasons of simplicity, and given that only DSC perfusion was used in the development of this thesis, DCE and ASL perfusion quantification will not be covered in this dissertation.

\section{DSC perfusion MRI}

DSC is the most frequently used technique for MRI perfusion of the brain. It relies on

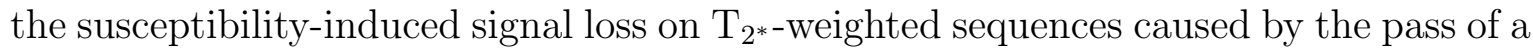
GBCA bolus through a capillary bed. A rapid repeated image acquisition is performed during the bolus injection, resulting into a series of images with the signal at each voxel representing the susceptibility-induced signal loss of the corresponding tissue, which is proportional to the amount of contrast present in the microvasculature. A mathematical model is then fit to the intensity-time signals to derive various perfusion parameters. The most commonly calculated parameters are relative Cerebral Blood Volume (rCBV), relative Cerebral Blood Flow (rCBF) and Mean Transit Time (MTT).

Let $S$ the susceptibility-induced signal loss observed at a given voxel. To quantify the rCBV, rCBF and MTT parameters, a GBCA concentration-time conversion of $S$ must be performed. It is normally assumed that the tissue concentration of the contrast agent is proportional to the change in $\mathrm{T}_{2^{*}}$ relaxation rate, i.e. $\Delta R 2^{*}$, by means of:

$$
\Delta R 2^{*}(t)=-\frac{1}{T E} \log \left(\frac{S(t)}{S_{0}}\right)=C(t)
$$

where $t$ indicates the time, TE refers to the echo time of the DSC sequence and $S_{0}$ refers to the intensity baseline of $S$.

Hence, DSC quantification allows to calculate the amount of GBCA tracer remaining in the tissue at each time step at which the $\Delta R 2^{*}(t)$ is measured. This is described by the so-called tissue impulse residue function, which is denoted as $R(t)$. Therefore, $R(t)$ represents the fraction of the tracer still present in the tissue at time $t$ after an instantaneous infinitesimal injection (i.e. a Dirac-delta function) of tracer into a tissue-feeding artery (Knutsson et al, 2010):

$$
R(t)=1-\int_{0}^{t} h(t) d t
$$

where $h(t)$ is the distribution of the capillary transit time. By definition, it is considered that $R(0)=1$, meaning that all the tracer is present in the tissue at $t=0$, and $R(\infty)=0$, meaning that the tracer lefts the tissue after a sufficiently long time (assuming an intact blood-brain barrier with no extravasation).

In practice, however, the arterial tracer bolus arrives at the tissue with a delay dependent on the circulatory path from the injection site to the tissue of interest. Consequently, the measured $\Delta R 2^{*}(t)$ signal in the tissue does not reflect the response to an instantaneous arterial input bolus, but to the convolution of a kernel given by $C B F \cdot R(t)$ and the $\triangle R 2^{*}(t)$ signal in the tissue-feeding artery, i.e. the so-called Arterial Input Function (AIF) (Rempp et al, 1994): 


$$
\begin{aligned}
C(t) & =C B F \cdot R(t) \otimes A I F(t) \\
& =C B F \int_{0}^{t} A I F(\tau) R(t-\tau) d \tau
\end{aligned}
$$

where $\otimes$ stands for the convolution product and CBF is the Cerebral Blood Flow.

From equation 2.3 we can rapidly observe that knowing the AIF, the CBF can be determined by deconvolution of the peak height of the $C B F \cdot R(t)$ signal, given that $R \in[0,1]$.

Dozens of models has been proposed in the literature to successfully estimate the CBF by deconvolution: fast truncated Singular Value Decomposition (SVD) family (e.g. sSVD, cSVD, oSVD (Ostergaard et al, 1996; Wu et al, 2003; Zanderigo et al, 2009)), nonlinear stochastic regularization (Zanderigo et al, 2009; Pillonetto and De Nicolao, 2010), Fourier-Hunt frequency-domain deconvolution (Ostergaard et al, 1996; Chen et al, 2005), wavelet thresholding (Connelly et al, 2006), classical Tikhonov regularization (Ostergaard et al, 1996; Zanderigo et al, 2009; Calamante et al, 2003), maximum likelihood estimation (Vonken et al, 1999), maximum entropy deconvolution (Drabycz et al, 2005), Gaussian process deconvolution (Andersen et al, 2002), or hierarchical Bayesian models (Schmid et al, 2007; Schmid, 2011).

In this thesis, the delay-insensitive oSVD method was employed to estimate the $\mathrm{CBF}$, as it has demonstrated to be robust to differences in tracer arrival times and to AIF selection variability. Following this approach, the deconvolution problem can be formulated as a matrix equation of the form $c=A b$ :

$$
\left[\begin{array}{c}
C\left(t_{0}\right) \\
C\left(t_{1}\right) \\
\vdots \\
C\left(t_{N-1}\right)
\end{array}\right]=\Delta t\left[\begin{array}{cccc}
A I F\left(t_{0}\right) & 0 & \ldots & 0 \\
A I F\left(t_{1}\right) & A I F\left(t_{0}\right) & \ldots & 0 \\
\vdots & \vdots & \ddots & \vdots \\
A I F\left(t_{N-1}\right) & A I F\left(t_{N-2}\right) & \ldots & A I F\left(t_{0}\right)
\end{array}\right]\left[\begin{array}{c}
R\left(t_{0}\right) \\
R\left(t_{1}\right) \\
\vdots \\
R\left(t_{N-1}\right)
\end{array}\right] C B F
$$

The above equation can be solved for $b$, with $b=R(t) \cdot C B F$. By decomposing $A=U \cdot S \cdot V^{T}$, with $U$ and $V$ orthogonal matrices and $S$ a non-negative square diagonal matrix, the inverse of $A$ can be expressed as $A^{-1}=V \cdot W \cdot U^{T}$ where $W=1 / S$ along the diagonal only where values are higher than a threshold (truncation of the diagonal for numerical stability reasons), and zero elsewhere. Therefore, we can estimate $C B F$ by solving for $b$ and calculating its maximum value:

$$
C B F=\max \left(V \cdot W \cdot U^{T} \cdot C\right)
$$

This procedure assumes that the $A I F$ always arrive before the voxel signal $C$, which in some situations may not be true (for example if the $A I F$ is not the true $A I F$ of the corresponding tissue). In such situations, the computation of the $C B F$ results in an incorrect estimation, typically an underestimation of the $C B F$, due to a shifting in the $R(t)$ function. 
As proposed by Wu et al (2003) circular deconvolution can be employed to solve this problem. By zero-padding the $N$ point-time series $A I F(t)$ and $C(t)$ to length $L$, with $L \geq 2 N$, time aliasing can be avoided. Thus, replacing also the matrix $A$ by a block-circulant matrix $D$ of the form:

$$
d_{i, j}= \begin{cases}a_{i, j}, & \text { for } j \leq i \\ a_{L+i-j, 0}, & \text { otherwise }\end{cases}
$$

and solving using the equation 2.5, the $C B F$ can be estimated safely also even if delay effects exist in the $A I F$.

The MTT can be calculated by Zierler's area-to-height relationship (Zierler Kenneth L., 1962), by the equation:

$$
M T T=\frac{\int_{0}^{\infty} R(t) d t}{\max [R(t)]}=\int_{0}^{\infty} R(t) d t
$$

According to the central volume theorem (Meier and Zierler, 1954; Weisskoff et al, 1993), the CBV can be calculated following the relation:

$$
C B V=C B F \cdot M T T
$$

Following this relation and combining equations 2.3 and 2.7, the CBV can also be estimated by:

$$
C B V=\frac{\int_{0}^{\infty} C(t) d t}{\int_{0}^{\infty} A I F(t) d t}
$$

\section{Leakage correction}

Computational kinetic models for DSC quantification explicitly assume that the GBCA remains in the intra-vascular space for the duration of the perfusion acquisition. However, this assumption is typically not valid for glioblastomas, since they often present blood-brain barrier breakdown. Therefore, GBCA extravasates to the extravascular interstitial space producing a distortion in the $\Delta R 2^{*}(t)$ signal that leads to an overestimation or underestimation of perfusion parameters if non leakage correction is performed. GBCA leakage can be manifested in $\Delta R 2^{*}(t)$ signals in generally two ways: the $\mathrm{T}_{2^{*}}$-dominant extravasation and the $\mathrm{T}_{1}$-dominant extravasation effects.

Numerous methods have been proposed in the literature to correct the leakage effect in perfusion, and the deviation it produces in the estimation of the perfusion markers: use of pre-dosing (Donahue et al, 2000), double-echo acquisitions (Vonken et al, 2000; Uematsu et al, 2001) or parametric modeling ( $\gamma$-variate fit of the $\Delta R 2^{*}(t)$ signals) (Thompson Howard K. et al, 1964). The most common and reference technique is the one proposed by Weisskoff et al (1994) and later elaborated by Boxerman et al (2006), which corrects the leaky signal by estimating its deviation from a non-leaky reference signal of the same patient.

Following Boxerman's method, the $\Delta R 2^{*}(t)$ observed at a given voxel can be determined as: 


$$
\Delta R 2^{*}(t) \approx K_{1} \overline{\Delta R 2^{*}}(t)-K_{2} \int_{0}^{t} \overline{\Delta R 2^{*}}\left(t^{\prime}\right) d t^{\prime}
$$

where $\overline{\Delta R 2^{*}}(t)$ stands for a whole-brain average signal in non-enhancing voxels, $K_{1}$ is a scale-constant of the whole-brain average signal to fit the observed signal at the corresponding voxel, and $K_{2}$ is the term that reflects the effects of the leakage (both $\mathrm{T}_{1}$ and $\mathrm{T}_{2}$ dominant effects). Therefore, by simple manipulation (please refer to Boxerman et al (2006) for more details), the leakage corrected signal can be determined as:

$$
\Delta R 2_{\text {corr }}^{*}(t)=\Delta R 2^{*}(t)+K_{2} \int_{0}^{t} \overline{\Delta R 2^{*}}\left(t^{\prime}\right) d t^{\prime}
$$

Both $K_{1}$ and $K_{2}$ can be determined by simple least squares fitting, and then corrected $\Delta R 2_{\text {corr }}^{*}(t)$ signals can be computed to quantify $C B V, C B F$ and $M T T$ as stated above. Figure 2.7 shows an example of the Boxerman's correction of a $\mathrm{T}_{2^{*-}}$ and a $T_{1}$-leaky curve.
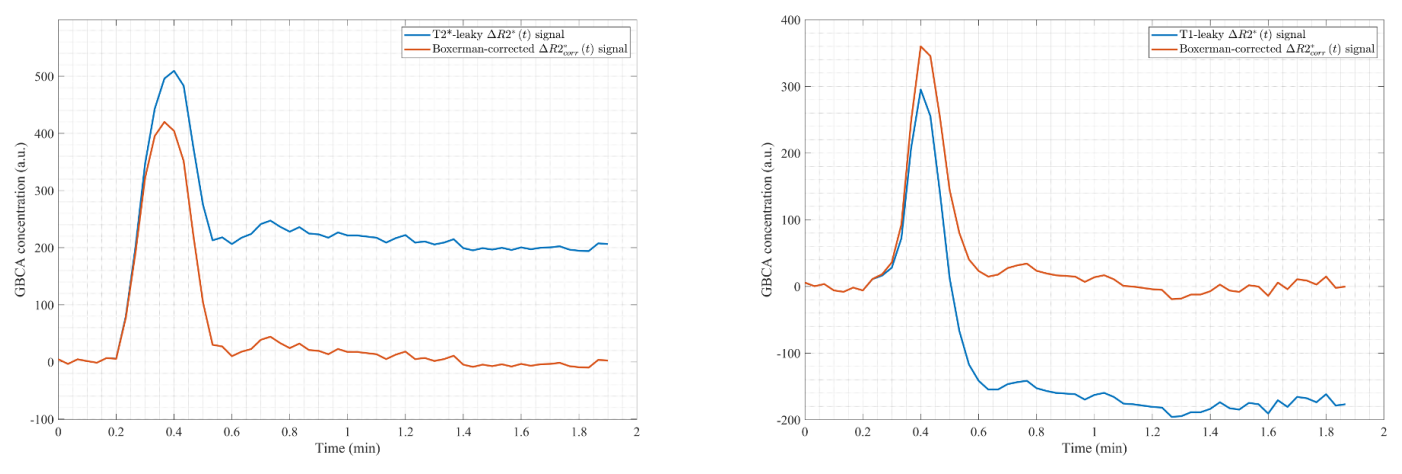

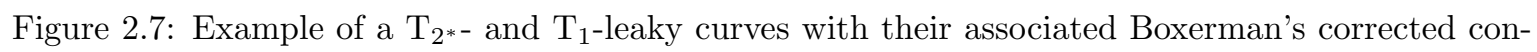
centration curves.

\section{Recirculation correction}

DSC quantification involves the kinetic analysis of the first pass of a intravenously injected GBCA. However, the $\Delta R 2^{*}(t)$ signal observed at each voxel primarily reflects the first pass of the tracer, but also the second wave of GBCA that recirculates into the tissue, after it has been shunted through the renal and coronary circulations and back into the heart. This second wave, affects the $\Delta R 2^{*}(t)$, preventing the signal from returning to its original baseline.

Hence, in order not to overestimate the perfusion parameters, typically the $C B V$, which is obtained from the integral or area under the $\Delta R 2^{*}(t)$ signal, the recirculation phase must be corrected. One of the most popular methods is to use a $\gamma$-variate fitting.

The $\gamma$-variate function commonly employed to describe the first pass of the GBCA is written as:

$$
S(t)=\mathcal{K} \cdot\left(t-t_{0}\right)^{\alpha} \exp ^{-\left(t-t_{0}\right) / \beta}
$$


where $\mathcal{K}$ is a scaling factor, $t_{0}$ is the bolus arrival time, and $\alpha$ and $\beta$ determine the shape and scale of the distribution.

Levenberg-Marquardt algorithm was employed in this thesis to perform the $\gamma$ variate fitting of all the $\Delta R 2^{*}(t)$ signals of the DSC perfusions. Levenberg-Marquardt algorithms searches for minimizing parameters in the form:

$$
\left(\hat{\mathcal{K}}, \hat{t_{0}}, \hat{\alpha}, \hat{\beta}\right)=\underset{\mathcal{K}, t_{0}, \alpha, \beta}{\arg \min } \sum_{t=0}^{N}\left[\Delta R 2^{*}(t)-S\left(t \mid \mathcal{K}, t_{0}, \alpha, \beta\right)\right]^{2}
$$

Figure 2.8 shows an example of a $\gamma$-variate fitting of a $\Delta R 2^{*}(t)$ signal.

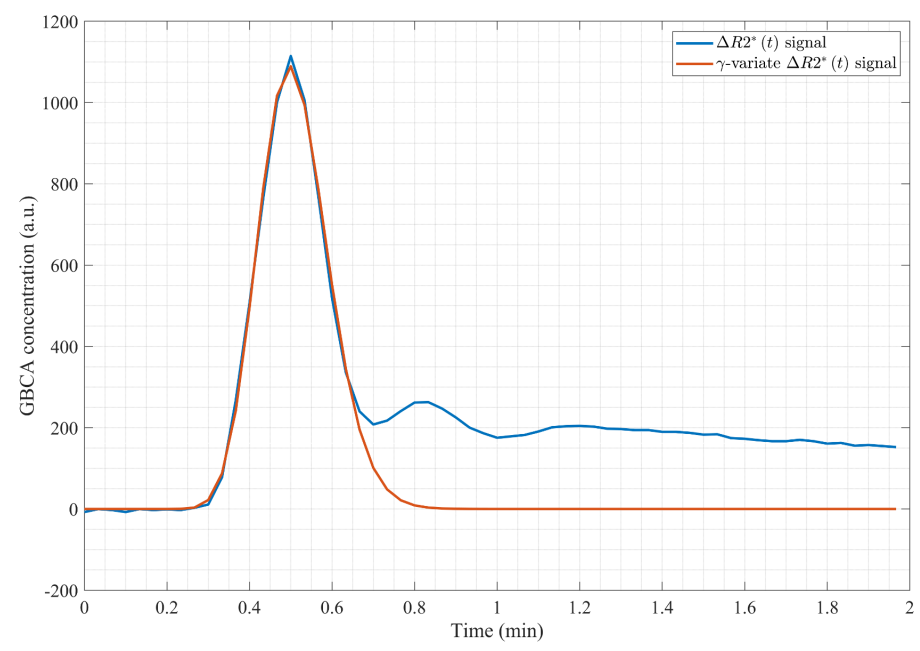

Figure 2.8: Example of a $\gamma$-variate fitting of a $\Delta R 2^{*}(t)$ signal.

\section{Contralateral white matter normalization}

Finally, the rCBV and rCBF parameters are obtained by normalizing the CBV and CBF quantities against the contralateral unaffected white matter value. This procedure has shown to successfully standardize perfusion values among individuals, regardless of their MRI protocol, hence allowing inter-patient multi-center studies (Álvarez Torres et al, 2019).

\subsection{Probability theory}

\subsubsection{Basic concepts}

In the following sections, the basic concepts of probability theory will be described to facilitate the reading and understanding of subsequent chapters and contributions of this thesis. 


\section{Random variables}

A random variable can be informally defined as a variable whose possible values are the outcomes of a random phenomenon. Thus, random variables conceptually represent an abstraction that allows addressing uncertainty in the measurement of quantities in real world scenarios.

More formally, a random variable $X: \Omega \rightarrow E$ is mathematically defined as a measurable function from a possible set of outcomes $\Omega$ to a measurable space $E$. Random variables can be of two types: discrete random variables, whose domain is a countable number of distinct values (typically $E=\mathbb{N}$ ); and continuous random variables, whose domain takes an infinite number of values in a predefined interval (typically $E=\mathbb{R}$ ). Usually, random variables are denoted by uppercase letters, e.g. $X$ and their realizations by lowercase letters, e.g. $x$. Hence, for example, a random variable that represents the volume of the brain in cubic centimeters in a population could denoted as $X$, and the particular measurement of a patient within this population could be denoted as $x=1273.6 \mathrm{~cm}^{3}$.

Random variables are usually governed by probability distributions that represent the likelihood that any of the possible values that the variable can take would occur. Such distributions constitute a fundamental principle to ML as they represent the mathematical approximation to deal with the uncertainty in the process of learning patterns from real data.

\section{Probability distribution}

A probability distribution is a mathematical function that describes the likelihood of obtaining the possible values that a random variable can assume. Given a random variable $X$, the probability that it takes the value $x$ is denoted as:

$$
p(X=x)
$$

where for the shake of simplicity we can assume $p(X=x) \equiv p(x)$.

Probability distributions are generally divided into two classes depending on the type of the random variable. For discrete random variables, the probability distribution is known as probability mass function (pmf), denoted as $f(x)$, and gives the probability mass that the random variable $X$ takes exactly the value $x$.

$$
p(x)=f(x) \rightarrow[0,1]
$$

Since the probability mass is distributed among all the possible outcomes that the discrete random variable can take, then:

$$
\sum_{x \in E} f(x)=1
$$

The cumulative distribution function (cdf), denoted as $F(x)$, is defined as the probability that the random variable $X$ takes values less than or equal to $x$, and takes the form: 


$$
F(x)=p(X \leq x)=\sum_{\substack{y \leq x \\ y \in E}} f(y)
$$

On the other hand, the probability distribution of a continuous random variable is known as probability density function ( $p d f)$, denoted also as $f(x)$. Since for continuous random variables there is an infinite number of values in any interval, the pdf for an exact specific value is not meaningful. Instead, the pdf for a continuous random variable is commonly defined over an interval $[a, b]$ in the form:

$$
p(a \leq X \leq b)=\int_{a}^{b} f(x) d x \rightarrow[0,1]
$$

For the shake of simplicity, we can assume that for continuous random variables $p(x)$ refers to the pdf of $x$ bounded to the infinitesimal interval $[x, x+d x]$ :

$$
p(x)=\int_{x}^{x+d x} f(y) d y
$$

Likewise the discrete case, the probability density is distributed among all the interval of possible values that the continuous random variable can take, which in the most general case is $[-\infty,+\infty]$, thus:

$$
\int_{-\infty}^{+\infty} f(x) d x=1
$$

The cdf for a continuous random variable is finally defined as:

$$
F(x)=\int_{-\infty}^{x} f(y) d y
$$

\section{The joint, marginal and conditional probability distributions}

In many situations we will manage more than one random variable that operates on the same probability space. In that situations, the joint, marginal and conditional probability distributions arise to model uncertain events from different interrelated events.

The joint probability distribution, denoted as $p(x, y)$, is defined as the probability distribution of two random variables operating in the same probability space whose outcomes occur simultaneously. The conditional distribution, denoted as $p(x \mid y)$, is defined as the probability distribution of a random variable when another random variable is known to have a particular value. Both distributions are closely related through the product rule, which takes the form:

$$
p(x, y)=p(x \mid y) p(y)=p(y \mid x) p(x)
$$

We can also relate these distributions with the marginal probability distribution through the sum rule, i.e. by integrating out a random variable that takes all the values in its subset. For discrete random variables the marginal probability distribution is defined as: 


$$
p(x)=\sum_{y} p(x, y)=\sum_{y} p(x \mid y) p(y)
$$

while for continuous random variables it is expressed as:

$$
p(x)=\int_{y} p(x, y) d y=\int_{y} p(x \mid y) p(y) d y
$$

\section{The Bayes' theorem and Bayes decision rule}

The Bayes' theorem plays a central role in ML as it provides a relationship between conditional and joint probability distributions of two random variables, which defines the so-called minimum-risk decision rule (Duda et al, 2000; Bishop, 2006). Therefore, the Bayes' theorem allows to take optimal decisions for uncertain events based on prior knowledge and conditions related to that events.

The Bayes' theorem, also named Bayes' rule or Bayes' law, is defined as:

$$
p(y \mid x)=\frac{p(x \mid y) p(y)}{p(x)}
$$

Under the context of a pattern recognition classification problem, these distributions have specific interpretations related to the degree of belief they give to the occurrence of different events. In this sense, the Bayes' formula can be informally expressed as:

$$
\text { posterior }=\frac{\text { likelihood } \times \text { prior }}{\text { evidence }}
$$

where each term semantically means:

$p(y \mid x)$ : posterior probability, that is the quantity that should be estimated and represents the degree of belief of observing the event (or class in a classification problem) $y$, after taking into account the evidence $x$.

$p(x \mid y)$ : likelihood distribution, it acts as a model of the random variable $Y$ that specifies the most probable outcome of $X$ given a specific state of $Y$.

$p(y)$ : prior distribution, it provides the degree of initial belief of observing the event $y$ of the random variable $Y$.

$p(x)$ : evidence distribution, it acts as a normalization term that provides the total degree of belief of the evidences $X$.

Note that the equation 2.25 can also be expressed in terms of joint probability distributions in the form:

$$
p(y \mid x)=\frac{p(x, y)}{\sum_{z} p(x, z)}
$$


from where it can be observed that the denominator acts as a normalization constant to ensure the conditional probability to sum 1.

Therefore, assuming $Y$ to be a discrete random variable that models the different classes of a pattern recognition classification problem, the Bayes' minimum-risk decision rule, sometimes also called minimum error-rate classifier, can be expressed as:

$$
\text { Decide } y \text { if } p(y \mid x)>p(z \mid x) \quad \forall z \neq y
$$

or more formally defined as:

$$
\hat{y}=\underset{y}{\arg \max } p(y \mid x)
$$

where $\hat{y}$ refers to the optimal class for the decision problem.

Following the Bayes' theorem, we can reformulate the equation 2.29 to:

$$
\begin{aligned}
\hat{y} & =\underset{y}{\arg \max } p(y \mid x) \\
& =\underset{y}{\arg \max } \frac{p(x \mid y) p(y)}{p(x)} \\
& =\underset{y}{\arg \max } p(x \mid y) p(y) \\
& =\underset{y}{\arg \max } p(x, y)
\end{aligned}
$$

from where it can be observed that we can drop out the $p(x)$ term as it is constant for the maximization (which is only dependent on $y$ ).

The previous result directly leads to the definition of the two main families of models within the ML classification problem: the generative models and the discriminative models. Generative models explicitly model the joint distribution $p(x, y)$, which semantically means that they learn a model to describe each class. By the opposite, the discriminative models directly learn the posterior distribution $p(y \mid x)$, which just models the decision boundaries between the different classes of the problem, ignoring the properties or characteristics of the classes.

Figure 2.9 illustrates both approaches, with the corresponding associated probability distributions and learning goals for each model.

\subsubsection{Probability distributions}

\section{The exponential family}

The exponential family of probability distributions is an unified set of distributions that can be expressed in the form:

$$
p(x ; \Theta)=h(x) \exp (\eta(\Theta) \cdot T(x)-A(\Theta))
$$

for some functions $h(x), \eta(\theta), T(x)$ and $A(\Theta)$; where $\Theta$ refers to the set of parameters of the distribution. 


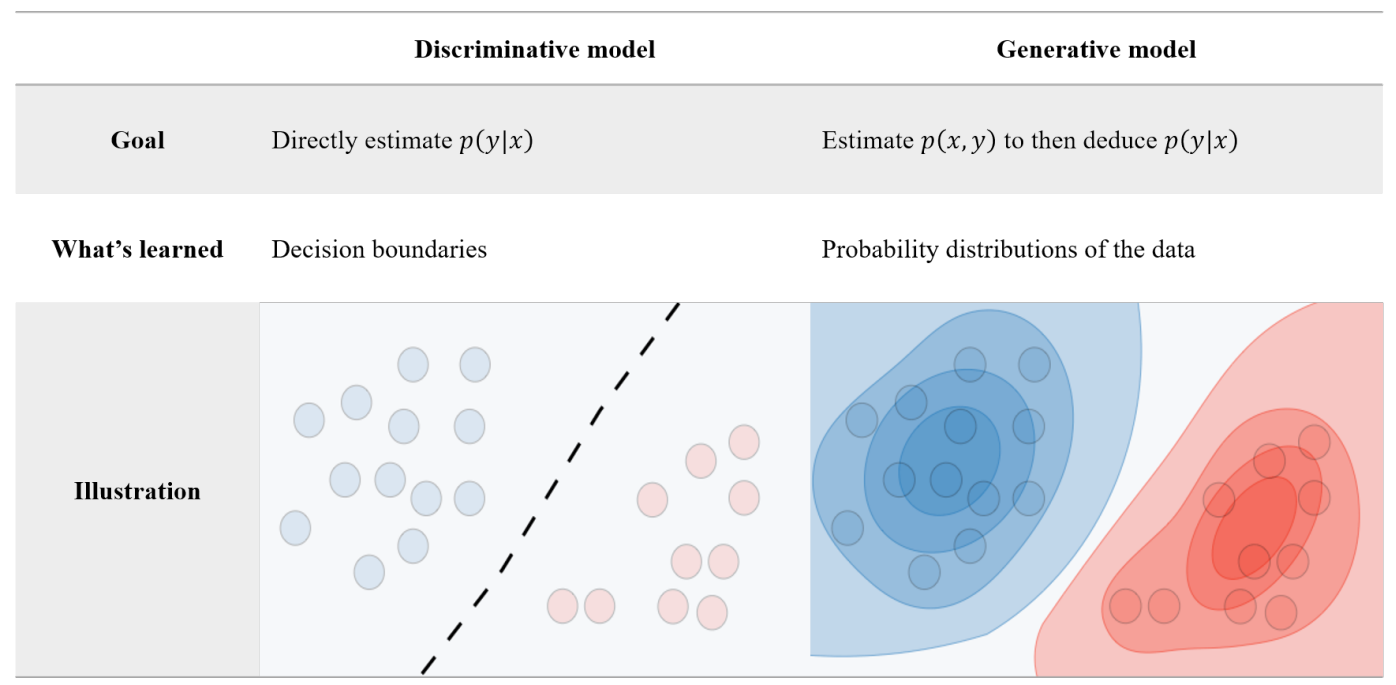

Figure 2.9: Summary of the goals and learning objectives of the generative and discriminative models for a pattern recognition classification problem. Image taken with kind permission from https:// stanford.edu/ shervine/teaching/cs-229/cheatsheet-supervised-learning.

The exponential family represents one of the most important families of distributions in statistics as it covers a wide range of distributions that naturally arise in many natural phenomena. Among others, the most important probability distributions of the exponential family are: the Normal, t-Student, Gamma, Multinomial and Dirichlet distributions, which are employed in some contributions of this thesis.

\section{The Normal distribution}

The most important distribution of the exponential family, and in statistics in general, is the Normal distribution. The Normal distribution, also called Gaussian distribution, is a continuous symmetric probability distribution defined in the range $[-\infty,+\infty]$, with parameters $\Theta=\left\{\mu, \sigma^{2}\right\}$, that takes the form:

$$
p\left(x ; \mu, \sigma^{2}\right)=\frac{1}{\sqrt{2 \pi \sigma^{2}}} \exp \left(-\frac{(x-\mu)^{2}}{2 \sigma^{2}}\right)
$$

where $\mu$ is referred as the mean and represents the central tendency or expected value of the random variable governed by the distribution; and $\sigma^{2}$ is referred as the variance and represents the degree of divergence of the realizations of the random variable from the central tendency.

The Normal distribution is also a fundamental distribution for probability theory as it arises from the central limit theorem, which states that the summation of independent random variables (properly normalized) tends to be normally distributed. This theorem has important implications since it allows to employ statistical methods designed for normal distributions to other problems involving non-normal distributions.

The generalization of the one-dimensional Normal distribution to the $d$-dimensional multivariate case with $d>1$ is defined as: 


$$
p(\mathbf{x} ; \mu, \Sigma)=\frac{1}{(2 \pi)^{n / 2}|\Sigma|^{1 / 2}} \exp \left(-\frac{1}{2}(\mathbf{x}-\mu)^{T} \Sigma^{-1}(\mathbf{x}-\mu)\right)
$$

where $\Sigma$ refers to the covariance matrix, and models the variance of each independent variable and the pair-wise covariance interactions between them.

The Normal distribution is typically denoted as $\mathcal{N}\left(\mu, \sigma^{2}\right)$ for the univariate case and $\mathcal{N}(\mu, \Sigma)$ for the multivariate case.

\section{The t-Student distribution}

The t-Student distribution is another continuous distribution of the exponential family that arises from the estimation of the mean of a population that is normally distributed. It is a symmetric zero-centered distribution, also defined in the range $[-\infty,+\infty]$, with parameters $\Theta=\{\nu\}$, that takes the form:

$$
p(x ; \nu)=\frac{\Gamma\left(\frac{\nu+1}{2}\right)}{\Gamma\left(\frac{\nu}{2}\right) \sqrt{\nu \pi}}\left(1+\frac{x^{2}}{\nu}\right)^{-\frac{\nu+1}{2}}
$$

with $\nu>0$ referring to the degrees of freedom of the distribution.

The t-Student distribution is very similar to the Normal distribution. In the limit $\nu \rightarrow+\infty$ the t-Student distribution exactly converges to a Normal typified distribution, while for values of $\nu \in[1,30]$ the distribution presents heavier and thicker tails, making it a natural choice for robust data modeling in presence of outliers.

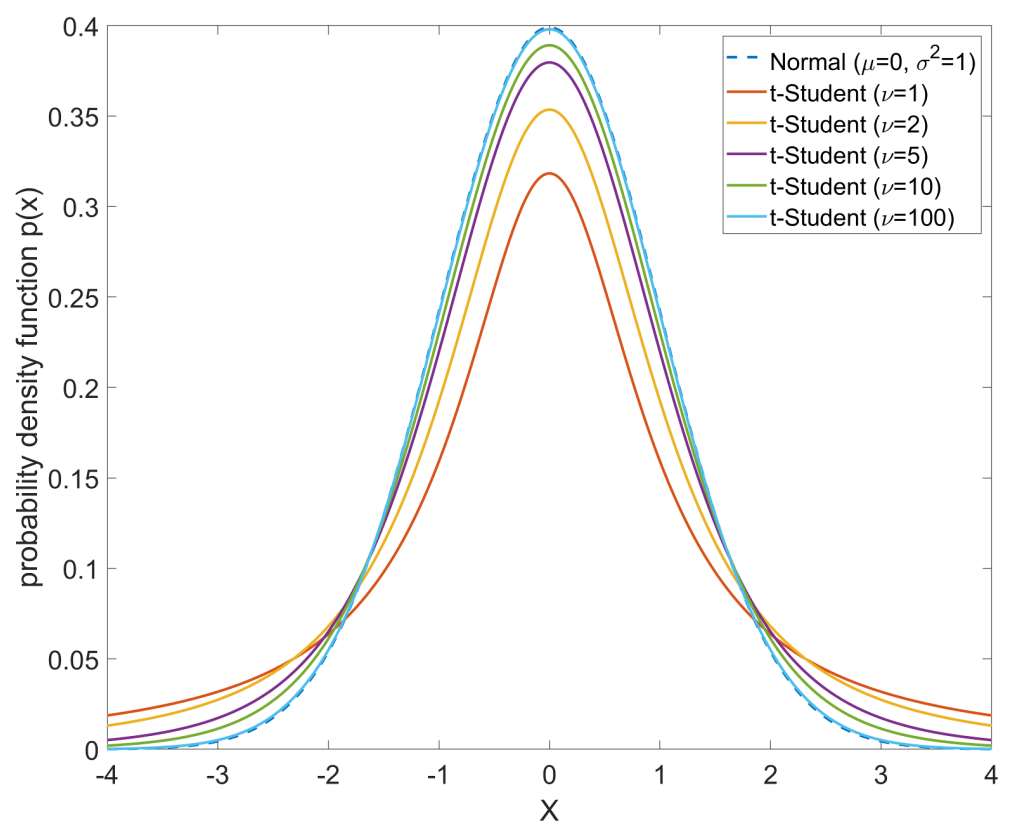

Figure 2.10: Comparison between probability density functions of a typified Normal distribution $\left(\mu=0, \sigma^{2}=1\right)$ and different instances of t-Student distributions with parameters $\nu=\{1,2,5,10,100\}$

The distribution can be generalized to a location-scale distribution (Bishop, 2006) by compounding a Normal distribution of mean $\mu$ and unknown variance with an 
inverse Gamma distribution placed over the variance with parameters $\alpha=\nu / 2$ and $\beta=\nu \sigma^{2} / 2$. The resulting density has the form:

$$
p\left(x ; \mu, \sigma^{2}, \nu\right)=\frac{\Gamma\left(\frac{\nu+1}{2}\right)}{\Gamma\left(\frac{\nu}{2}\right) \sqrt{\nu \pi \sigma^{2}}}\left(1+\frac{1}{\nu} \frac{(x-\mu)^{2}}{\sigma^{2}}\right)^{-\frac{\nu+1}{2}}
$$

The generalization of the one-dimensional t-Student distribution to the $d$-dimensional multivariate case is defined as:

$$
p(\mathbf{x} ; \mu, \Sigma, \nu)=\frac{\Gamma\left(\frac{\nu+d}{2}\right)}{\Gamma\left(\frac{\nu}{2}\right)(\nu \pi)^{d / 2}|\Sigma|^{1 / 2}}\left(1+\frac{1}{\nu}(\mathbf{x}-\mu)^{T} \Sigma^{-1}(\mathbf{x}-\mu)\right)^{-\frac{\nu+d}{2}}
$$

where $d$ refers to the dimensions of the random variable and $\Sigma$ refers to the scale or shape matrix, which in general is not equivalent to the covariance matrix of the Normal distribution.

The t-Student distribution is typically denoted as $\mathcal{S} t(\nu)$, or $\mathcal{S} t\left(\mu, \sigma^{2}, \nu\right)$ and $\mathcal{S} t(\mu, \Sigma, \nu)$ for the location-scale univariate and multivariate versions respectively.

\section{The Gamma distribution}

The Gamma distribution is another continuous probability distribution of the exponential family, which is very common in the biomedical field as many biological phenomena follow a Gamma distribution. It is a positive-only right-skewed distribution defined in the range $(0,+\infty]$, with parameters $\Theta=\{k, \theta\}$, that takes the form:

$$
p(x ; k, \theta)=\frac{1}{\Gamma(k) \theta^{k}} x^{k-1} \exp \left(-\frac{x}{\theta}\right)
$$

where $k$ is referred to as the shape parameter and $\theta$ is referred to as the scale parameter.

Such distribution is widely employed to model time-dependent biological phenomena that have a natural minimum of 0 , such as the MR signal decay produced by the first pass of the bolus of a paramagnetic contrast agent intravenously injected to a patient.

The generalization of the univariate Gamma distribution to the multivariate case is the Wishart distribution. This distribution is defined for positive-definite $(d \times d)$ matrices that represents the scatter matrix of two $d$-variate Normal-distributed random variables, which falls far outside the scope of this thesis.

The Gamma distribution is typically denoted as $\mathcal{G}(k, \theta)$.

\section{The Multinomial distribution}

The Multinomial distribution is a discrete probability distribution that models the probability of observing each of $K$ different outcomes in an experiment involving $n$ repeated trials. Therefore, the distribution governs a discrete random variable $X$, whose realizations are vectors of the form $\mathbf{x}=\left(x_{1}, \ldots, x_{K}\right)$, with $x_{k} \in\{0, \ldots, n\}$ representing the number of times the $k^{\text {th }}$ outcome has been observed after the $n$ trials. 
The Multinomial distribution has the parameters $\Theta=\{\mathbf{p}, n\}$, with $\mathbf{p}=\left(p_{1}, \ldots, p_{K}\right)$, representing the prior probability of observing each of the $K$ possible outcomes before the trials, and $n$ indicating the number of trials. The pmf of the distribution takes the form:

$$
p(\mathbf{x} ; \mathbf{p}, n)= \begin{cases}\frac{n !}{x_{1} ! \ldots x_{K} !} p_{1}^{x_{1}} \ldots p_{K}^{x_{K}} & \text { when } \sum_{i=1}^{K}=n \\ 0 & \text { otherwise }\end{cases}
$$

The Multinomial distribution is the generalization of several discrete distributions, those ones taking specific values for $k$ and $n$ parameters. Thus, the Multinomial distribution converts to the Bernoulli distribution when $k=2$ and $n=1$; to the Binomial distribution when $k=2$ and $n>1$; and to the Categorical distribution when $k>1$ and $n=1$.

The Multinomial distribution can also be expressed using the Gamma function as:

$$
p(\mathbf{x} ; \mathbf{p})=\frac{\Gamma\left(\sum_{k} x_{k}+1\right)}{\prod_{k} \Gamma\left(x_{k}+1\right)} \prod_{k=1}^{K} p_{k}^{x_{k}}
$$

whose form is very similar to the Dirichlet distribution, which is its conjugate prior.

The Multinomial distribution is typically denoted as $\operatorname{Mult}(\mathbf{p}, n)$.

\section{The Dirichlet distribution}

The Dirichlet distribution is a continuous probability distribution typically used in Bayesian statistics as conjugate prior of the Multinomial distribution. It is the multivariate generalization of the Beta distribution, which is commonly used to model prior knowledge about the probability of an event. Therefore, the Dirichlet distribution governs a random variable $X$ whose outcomes are the realizations of a Multinomial distribution in the form $\mathbf{x}=\left(x_{1}, \ldots, x_{K}\right)$, with $x_{k} \in[0,1]$. The distribution has the parameters $\Theta=\{\alpha\}$ and is defined as:

$$
p(\mathbf{x} ; \alpha)=\frac{\Gamma\left(\sum_{k} \alpha_{k}\right)}{\prod_{k} \Gamma\left(\alpha_{k}\right)} \prod_{k=1}^{K} x_{k}^{\left(\alpha_{k}-1\right)}
$$

with $\alpha$ called the concentration parameter.

As stated above, the Dirichlet distribution is the conjugate prior of the Multinomial distribution, which means that if a random variable $X$ follows a Multinomial distribution, and we assume a prior Dirichlet distribution over its parameter $\mathbf{p}$, then the posterior distribution of $\mathbf{p}$ is also Dirichlet distributed. Under the Bayesian statistics, this relationship leads to a powerful mechanism to, given a new observation $\mathbf{x}$, update the parameter $\mathbf{p}$ in a pure algebraic manner, without recalculating the joint probability distribution $p(\mathbf{x}, \mathbf{p})$, which normally remains intractable.

The Dirichlet distribution is typically denoted as $\operatorname{Dir}(\alpha)$. 


\subsubsection{Parameter estimation}

Statistical inference is the process of deducing properties and making generalizations about a population, by estimating the parameters of an underlying probability distribution used to model observations taken from that population. Therefore, statistical modeling typically consists of two steps: 1 ) choosing the distribution that best fits the data, and 2) determining the parameters $\Theta$ of the distribution.

However, $\Theta$ is generally considered unobservable, as the whole population cannot be observed either. Therefore, an estimation of the parameters $\hat{\Theta}$ is usually calculated, based on a random sample drawn from the population. The most typical analytical methods of estimating the parameters of a probability distribution given its pdf (or pmf) are: Maximum Likelihood Estimation (MLE), Maximum A Posteriori (MAP) estimation and Bayesian inference, of which the first two are briefly described below as they are extensively used in this thesis. On the other hand, numerical optimization methods such as the Expectation-Maximization (EM) algorithm are also typically employed to estimate the parameters of complex models that do not have closed-form analytical solution. The EM algorithm is also discussed below as it is extensively used in this thesis for several contributions.

\section{Maximum Likelihood Estimation}

The Maximum Likelihood Estimation (MLE) focuses on obtaining the best distribution parameters $\hat{\Theta}$ by maximizing the likelihood function $\mathcal{L}(\Theta ; x)$. This approach can be semantically interpreted as "maximizing the probability of observing the random sample $X$ given a current guess about the parameters $\Theta$ of the model".

Let $X=\left(\mathbf{x}^{1}, \ldots, \mathbf{x}^{N}\right)$ a set of observations of a random variable. Formally, the MLE estimate is defined as:

$$
\begin{aligned}
\hat{\Theta}_{M L E} & =\underset{\Theta}{\arg \max } \mathcal{L}(\Theta ; X) \\
& =\underset{\Theta}{\arg \max } p(X ; \Theta) \\
& =\underset{\Theta}{\arg \max } \prod_{i} p\left(\mathbf{x}^{i} ; \Theta\right)
\end{aligned}
$$

Differentiation is employed to maximize $\mathcal{L}(\Theta ; X)$, by setting the partial derivatives of the function with respect to each parameter to zero. However, maximizing $\mathcal{L}(\Theta ; X)$ is hard as it involves a product over all realizations of $X$. Typically, natural logarithm is taken to simplify the expression in various senses: first, natural logarithm is a monotonically increasing function so the maximum value of $\mathcal{L}(\Theta ; X)$ occurs at the same point as the maximum value of $\log \mathcal{L}(\Theta ; X)$; next, the logarithm of the product converts to the sum of the logarithms, which is far easier to maximize than the product since the derivative of a sum is the sum of derivatives; additionally, probability distributions involving the exponential function take benefit from the logarithm to simplify the expression to be maximized; finally, computing a sum of log-derivatives is computationally more stable than calculating a product of probabilities, which quickly tend to zero leading to numerical representation problems. 
Therefore, the log-likelihood is usually maximized in MLE, which is defined as:

$$
\begin{aligned}
& \hat{\Theta}_{M L E}=\underset{\Theta}{\arg \max } \log \mathcal{L}(\Theta ; X) \\
& =\underset{\Theta}{\arg \max } \log p(X ; \Theta) \\
& =\underset{\Theta}{\arg \max } \log \prod_{i} p\left(x^{i} ; \Theta\right) \\
& =\underset{\Theta}{\arg \max } \sum_{i} \log p\left(x^{i} ; \Theta\right)
\end{aligned}
$$

\section{Maximum A Posteriori}

Maximum A Posteriori (MAP) estimation is closely related to MLE in the sense that both look for the parameters that equal to the mode of a distribution (the maximum of the distribution). However, while MLE maximizes the likelihood (or log-likelihood) distribution of the parameters $\Theta$, MAP estimation maximizes the posterior distribution of $\Theta$ with respect to the observations $X$. Therefore, in this case, MAP estimate can be semantically interpreted as "maximizing the probability of a specific setting of the $\Theta$ parameters of the model given the current observations of the random variable $X$ ". In this sense, MAP is also closely related to Bayesian estimation, since it considers $\Theta$ parameters as a random variable that in turn is governed by some distribution with its own parameters. Therefore, assuming $\Theta$ as a random variable and taking the Bayes' rule:

$$
\begin{aligned}
p(\Theta \mid X) & =\frac{p(X \mid \Theta) p(\Theta)}{p(X)} \\
& \propto p(X \mid \Theta) p(\Theta)
\end{aligned}
$$

where we can ignore the normalizing constant as we are strictly speaking about normalization, so proportionality is sufficient. Hence, the MAP estimate is defined as:

$$
\begin{aligned}
\hat{\Theta}_{M A P} & =\underset{\Theta}{\arg \max } \log \mathcal{L}(X \mid \Theta) \\
& =\underset{\Theta}{\arg \max } \log p(\Theta \mid X) \\
& =\underset{\Theta}{\arg \max } \log (p(X \mid \Theta) p(\Theta)) \\
& =\underset{\Theta}{\arg \max } \log p(X \mid \Theta)+\log p(\Theta) \\
& =\underset{\Theta}{\arg \max } \sum_{i} \log p\left(x^{i} \mid \Theta\right)+\log p(\Theta)
\end{aligned}
$$

from where it can be clearly observed that MAP is an augmentation of MLE that allows to introduce a density $p(\Theta)$ over the parameters of the model, to inject initial beliefs and/or prior knowledge about them. Moreover, it can also be quickly deduced that 
MLE is a particular case of MAP estimation when $p(\Theta)$ is assumed to be constant. Thus, MAP can also be thought as a regularization/generalization of the MLE method.

As in MLE, differentiation is employed to maximize $\log \mathcal{L}(X \mid \Theta)$ and obtain closedform solutions for the estimation of the parameters of the model. At this point, conjugate priors, briefly introduced in section 2.3.2, become important when modeling $p(\Theta)$, since assuming an adequate distribution for $p(\Theta)$ may be crucial to update the parameters in a straightforward manner.

\section{Expectation-Maximization algorithm}

As stated above, MLE and MAP estimation consist in the maximization of the likelihood and posterior functions respectively, by mathematical differentiation. However, setting partial derivatives for the parameters of many complex models usually does not find closed-form expressions, hence preventing direct analytical solutions.

A common way to deal with the complexity in optimizing parameters of models that do not have closed form solution is to introduce a set of latent variables $Z$ that allow the model to be formulated in a more tractable way. Therefore, defining a joint distribution $p(X, Z ; \Theta)$ over the augmented space of observed and latent variables allows to estimate the parameters of the model $\Theta$ in a more straightforward manner. Then, once $\Theta$ is estimated, the distribution of the observed variables $p(X ; \Theta)$ can be obtained by marginalization. The EM algorithm takes advantage of such idea.

The EM algorithm, formalized in 1977 by Arthur Dempster, Nan Laird and Donald Rubin (Dempster et al, 1977), provides an iterative numerical optimization framework for the MLE and MAP estimate of models involving latent variables, when a closedform analytical solution is not possible. The algorithm relies on the introduction of an informative latent variables that allow the model to be reformulated in such a way that a closed-form solution for the estimation of the parameters can be found.

Of course, these latent variables $Z$ are unknown quantities that prevent a direct estimation of the model, so maximizing $\log \mathcal{L}(\Theta ; X, Z)$ (for the MLE example case) is not possible. However, some state of knowledge about the value of $Z$ can be obtained by computing its posterior distribution given the observations $X$ and a guess about the parameters of the model $\tilde{\Theta}$, i.e. $p(Z \mid X ; \tilde{\Theta})$. Therefore, instead of maximizing $\log \mathcal{L}(\Theta ; X, Z)$ the EM algorithm maximizes an auxiliary function, typically referred as the $\mathcal{Q}$-function, which is the expected value of $\log \mathcal{L}(\Theta ; X, Z)$ under this posterior distribution. That is:

$$
\begin{aligned}
\hat{\Theta}_{M L E} & =\underset{\Theta}{\arg \max } \mathcal{Q}(\Theta ; \tilde{\Theta}) \\
& =\underset{\Theta}{\arg \max } \mathbb{E}_{p(Z \mid X ; \tilde{\Theta})}[\log \mathcal{L}(\Theta ; X, Z)] \\
& =\underset{\Theta}{\arg \max } \sum_{Z} p(Z \mid X ; \tilde{\Theta}) \log p(X, Z ; \Theta) \\
& =\underset{\Theta}{\arg \max } \sum_{i} p\left(z^{i} \mid x^{i} ; \tilde{\Theta}\right) \log p\left(x^{i}, z^{i} ; \Theta\right)
\end{aligned}
$$


This formulation quickly suggest an iterative scheme based on alternating between computing the conditional expectations of the latent variables given the observations, i.e. computing $p(Z \mid X ; \tilde{\Theta})$, and updating the parameters of the model via the closedform solutions obtained thanks to the augmented formulation $p(X, Z ; \Theta)$.

Therefore, the general scheme of the EM algorithm (for the MLE case) is:

Initialization: Choose an initial setting for $\tilde{\Theta}$.

Expectation step: Evaluate the $\mathcal{Q}$-function:

$$
\mathcal{Q}(\Theta ; \tilde{\Theta})=\mathbb{E}_{p(Z \mid X ; \tilde{\Theta})}[\log \mathcal{L}(\Theta ; X, Z)]
$$

which actually means estimating the unknown quantity $p(Z \mid X ; \tilde{\Theta})$

Maximization step: Maximize the $\mathcal{Q}$-function:

$$
\hat{\Theta}_{M L E}=\underset{\Theta}{\arg \max } \mathcal{Q}(\Theta ; \tilde{\Theta})
$$

which consist of updating the parameters $\Theta$ of the model based on $p(Z \mid X ; \tilde{\Theta})$

Convergence: Stop if $\mathcal{L}(\hat{\Theta} ; X)-\mathcal{L}(\tilde{\Theta} ; X) \leq \epsilon$; otherwise $\tilde{\Theta}=\hat{\Theta}$ and go to Expectation step.

Parameter estimation via EM algorithm is only guaranteed to converge to a local maxima or to a saddle point of the function under maximization. Convergence to the global maxima is not assured since the algorithm is only guaranteed to iteratively increase the likelihood (or posterior) function after each iteration. Hence, optimizing multi-modal functions can easily lead to local maximas, also conditioned by the initialization of $\tilde{\Theta}$. An in-depth dissertation on the convergence properties of the EM was made by professor $\mathrm{Wu}(1983)$.

In the following chapters, several applications of MLE and MAP parameter estimation of complex models via EM will be presented. 


\subsection{Mixture Models}

So far we have seen that probability distributions give us a mathematical form to describe the properties of a population represented by a random variable. However, assuming that each observation is drawn from a single unimodal distribution leads to a very simplistic scenario that generally does not apply in real-world situations. In fact, real-world data typically present more complex patterns such as multi-modality, sparsity, noise or presence of outliers. In this sense, models capable of capturing such variability are required to describe the data in a more reliable sense.

In the following sections, three different families of mixture models grouped by their structured or non-structured nature will be discussed.

\subsubsection{Finite Mixture Model}

Finite Mixture Models (FMMs) are probabilistic models that model a random variable as a convex combination of pdfs. Therefore, FMM provides a statistical formal framework to describe heterogeneous data trough a weighted sum of single distributions, each one representing a sub-population within a dataset.

Let $X=\left(\mathbf{x}^{1}, \ldots, \mathbf{x}^{N}\right)$ a set of observations of a random variable, where $\mathbf{x}^{i} \in \mathbb{R}^{d}$. A FMM of $K$ components assumes a pdf over $X$ in the form:

$$
\begin{aligned}
p(X ; \Theta) & =\prod_{i=1}^{N} p\left(\mathbf{x}^{i} ; \Theta\right) \\
& =\prod_{i=1}^{N} \sum_{j=1}^{K} \pi_{j} \phi\left(\mathbf{x}^{i} ; \Theta_{j}\right)
\end{aligned}
$$

where $\Theta=\left\{\Theta_{1}, \ldots, \Theta_{K}, \pi_{1}, \ldots, \pi_{K}\right\}$ are the parameters of the model, with $\left\{\Theta_{j}, \pi_{j}\right\}$ the parameters of the $j^{\text {th }}$ component of the mixture modeled by the pdf $\phi\left(\mathbf{x}^{i} ; \Theta_{j}\right)$. The parameters $\left\{\pi_{1}, \ldots, \pi_{K}\right\}$ are typically called mixing coefficients and can be seen as the prior probability of each component of the mixture describing the data. Therefore, it follows that $0 \leq \pi_{j} \leq 1$ and:

$$
\sum_{j=1}^{K} \pi_{j}=1
$$

Due to its mathematical tractability, Gaussian (or t-Student) distributions are typically employed to model the data, thus $\phi\left(\mathrm{x}^{i} ; \Theta_{j}\right) \sim \mathcal{N}\left(\mathrm{x}^{i} ; \mu_{j}, \Sigma_{j}\right)$. Figure 2.11 illustrates the pdf of a 3-component Gaussian Mixture Model (GMM) (in purple), with their associated Normal distributions (blue, red and yellow).

A common way to estimate the parameters of a FMM could be via MLE. From 2.46 we can set: 


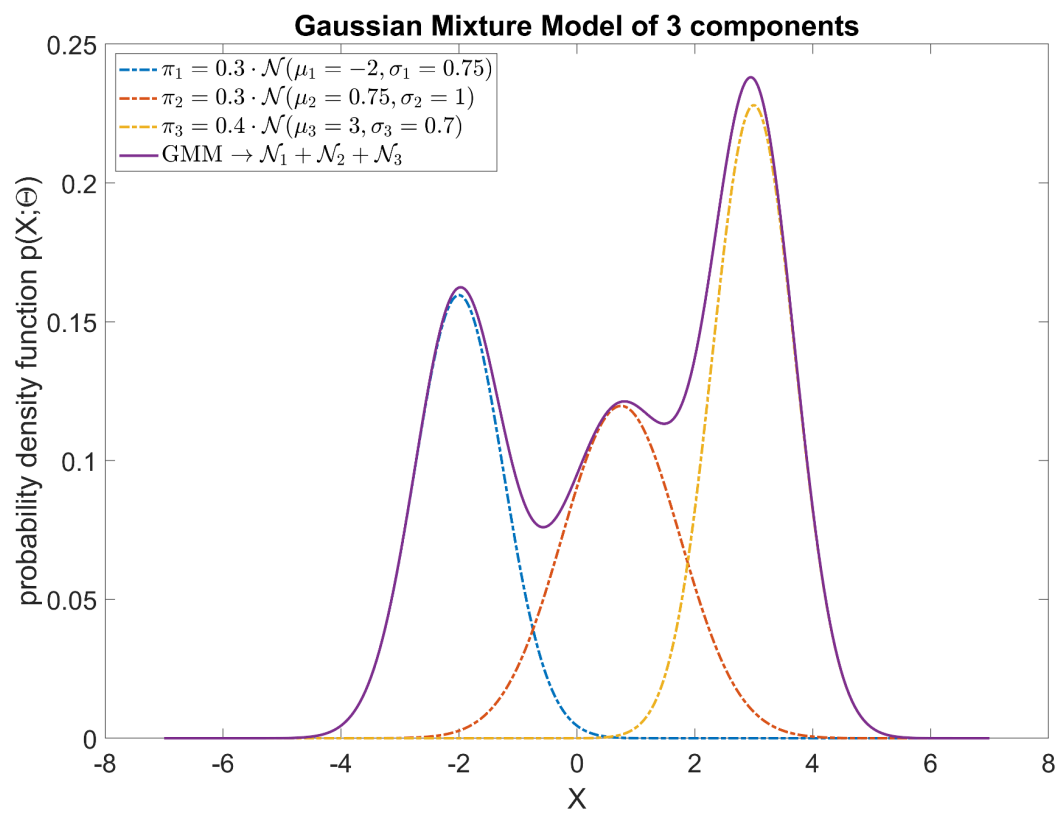

Figure 2.11: Probability density functions of a 3-component GMM with their associated Normal distributions.

$$
\begin{aligned}
\hat{\Theta}_{M L E} & =\underset{\Theta}{\arg \max } \log \mathcal{L}(\Theta ; X) \\
& =\underset{\Theta}{\arg \max } \log p(X ; \Theta) \\
& =\underset{\Theta}{\arg \max } \log \prod_{i=1}^{N} \sum_{j=1}^{K} \pi_{j} \phi\left(\mathbf{x}^{i} ; \Theta_{j}\right) \\
& =\underset{\Theta}{\arg \max } \sum_{i=1}^{N} \log \sum_{j=1}^{K} \pi_{j} \phi\left(\mathbf{x}^{i} ; \Theta_{j}\right)
\end{aligned}
$$

from where we can immediately see that the maximization is very complex due to the summation over $K$ inside the logarithm. In fact, there is no closed-form analytical solution for the MLE (nor MAP estimate) of a FMM. In this sense, alternative methods are required to estimate the parameters of these models in a reliable but affordable manner.

\section{EM inference for FMM}

As stated in chapter 2.3.3, a common way to deal with the complexity in optimizing parameters of models that do not have closed form solution is to introduce a set of latent variables $Z$ that allow the model to be formulated in a more tractable way. Under the FMM's point of view, a latent variable $Z$ is typically introduced indicating the component of the mixture to which the observation belongs to. Formally, 
let $Z=\left(\mathbf{z}^{1}, \ldots, \mathbf{z}^{N}\right)$ a random variable where $\mathbf{z}^{i} \in\{0,1\}^{K}$ represents a binary $K$ dimensional one-hot encoding variable, where $z_{k}^{i}=1$ indicates that the $i^{\text {th }}$ observation has originated by the $k^{t h}$ component of the mixture, and hence $z_{j}^{i}=0, \forall j \neq k$. Under this assumption, we can reformulate the FMM by defining a joint density over the latent and observed variables in the form:

$$
p(X, Z ; \Theta)=\prod_{i=1}^{N} \prod_{j=1}^{K}\left(\pi_{j} \phi\left(\mathbf{x}^{i} ; \Theta_{j}\right)\right)^{z_{j}^{i}}
$$

where we assume that $Z$ follows a Multinomial unit-length distribution given by equation 2.38 with $n=1$ (i.e. Categorical), i.e.:

$$
z^{i} \sim \operatorname{Cat}_{K}\left(\pi^{i}\right)
$$

The MLE estimate of this augmented FMM can be expressed as:

$$
\begin{aligned}
\hat{\Theta}_{M L E} & =\underset{\Theta}{\arg \max } \log \mathcal{L}(\Theta ; X, Z) \\
& =\underset{\Theta}{\arg \max } \log p(X, Z ; \Theta) \\
& =\underset{\Theta}{\arg \max } \log \prod_{i=1}^{N} \prod_{j=1}^{K}\left(\pi_{j} \phi\left(\mathbf{x}^{i} ; \Theta_{j}\right)\right)^{z_{j}^{i}} \\
& =\underset{\Theta}{\arg \max } \sum_{i=1}^{N} \sum_{j=1}^{K} \log \left(\pi_{j} \phi\left(\mathbf{x}^{i} ; \Theta_{j}\right)\right)^{z_{j}^{i}} \\
& =\underset{\Theta}{\arg \max } \sum_{i=1}^{N} \sum_{j=1}^{K} z_{j}^{i} \log \left(\pi_{j} \phi\left(\mathbf{x}^{i} ; \Theta_{j}\right)\right) \\
& =\underset{\Theta}{\arg \max } \sum_{i=1}^{N} \sum_{j=1}^{K} z_{j}^{i}\left(\log \pi_{j}+\log \phi\left(\mathbf{x}^{i} ; \Theta_{j}\right)\right)
\end{aligned}
$$

which is far easier to optimize than the original FMM. In fact, closed-form solutions can be found for all the parameters of the model depending on the form of $\phi\left(x^{i} ; \Theta_{j}\right)$.

Optimizing $\pi_{j}$ requires considering the constraint that $\sum_{k} \pi_{k}=1$, which can be easily achieved by using Lagrange multipliers. Therefore, setting the derivatives of $\log \mathcal{L}(\Theta ; X, Z)$ with respect $\pi_{j}$ and $\lambda$ Lagrange multiplier yield:

$$
\begin{aligned}
\frac{1}{\pi_{j}} \sum_{i=1}^{N} z_{j}^{i}+\lambda & =0 \\
\sum_{k=1}^{K} \pi_{k}-1 & =0
\end{aligned}
$$

Solving the system of equations and rearranging for $\pi_{j}$ provides: 


$$
\pi_{j}=\frac{\sum_{i=1}^{N} z_{j}^{i}}{\sum_{i=1}^{N} \sum_{k=1}^{K} z_{k}^{i}}
$$

Note that the denominator of the above expression (2.53) is actually equal to $N$, since it is a summation of $\mathrm{N}$ one-hot encoding variables $\mathbf{z}^{i}$, which take a value 1 for one of their components and 0 for the rest.

For the most typical case of FMM where $\phi\left(x^{i} ; \Theta_{j}\right) \sim \mathcal{N}\left(x^{i} ; \mu_{j}, \Sigma_{j}\right)$ (i.e. GMM), means and covariance matrices must be optimized. Setting the derivatives of $\log \mathcal{L}(\Theta ; X, Z)$ with respect to the mean yields:

$$
\sum_{i=1}^{N} z_{j}^{i} \Sigma_{j}^{-1}\left(\mathbf{x}^{i}-\mu_{j}\right)=0
$$

where rearranging:

$$
\mu_{j}=\frac{1}{\sum_{i=1}^{N} z_{j}^{i}} \sum_{i=1}^{N} z_{j}^{i} \mathbf{x}^{i}
$$

and solving for the covariance matrix yields:

$$
\sum_{i=1}^{N} z_{j}^{i}\left(-\frac{1}{2} \Sigma_{j}^{-1}+\frac{1}{2}\left(\mathbf{x}^{i}-\mu_{j}\right)^{T} \Sigma_{j}^{-2}\left(\mathbf{x}^{i}-\mu_{j}\right)\right)=0
$$

where rearranging:

$$
\Sigma_{j}=\frac{1}{\sum_{i=1}^{N} z_{j}^{i}} \sum_{i=1}^{N} z_{j}^{i}\left(\mathbf{x}^{i}-\mu_{j}\right)\left(\mathbf{x}^{i}-\mu_{j}\right)^{T}
$$

Up to this point we have seen that assuming that we have both the observations and latent variables $\{X, Z\}$, typically referred as the complete dataset, the MLE (and also the MAP) estimate find closed-form solutions for the parameters of the FMM. However, actually, we only have the observations $X$ (i.e. the incomplete dataset), so, as previously described in section 2.3.3, the only information we can get from the latent variable $Z$ is given by its posterior distribution conditioned to the observations $X$ and to a current estimate of the parameters of the model $\tilde{\Theta}$, i.e. $p(Z \mid X ; \tilde{\Theta})$. Following the Bayes' rule it is given by:

$$
p\left(z_{j}^{i}=1 \mid \mathbf{x}^{i} ; \tilde{\Theta}\right)=\gamma_{j}^{i}=\frac{\pi_{j} \phi\left(\mathbf{x}^{i} ; \tilde{\Theta}_{j}\right)}{\sum_{k} \pi_{k} \phi\left(\mathbf{x}^{i} ; \tilde{\Theta}_{k}\right)}
$$


Therefore, because it is not possible to compute the MLE of $\log \mathcal{L}(\Theta ; X, Z)$ (due to we only can observe $X$ ), we just can compute its expected value under the posterior distribution $p(Z \mid X ; \tilde{\Theta})$, which yields the so-called $Q$-function:

$$
\begin{aligned}
\hat{\Theta}_{M L E} & =\underset{\Theta}{\arg \max } \mathcal{Q}(\Theta ; \tilde{\Theta}) \\
& =\underset{\Theta}{\arg \max } \mathbb{E}_{p(Z \mid X ; \tilde{\Theta})} \log \mathcal{L}(\Theta ; X, Z) \\
& =\underset{\Theta}{\arg \max } \sum_{Z} p(Z \mid X ; \tilde{\Theta}) \log p(X, Z ; \Theta) \\
& \cdots \\
& =\underset{\Theta}{\arg \max } \sum_{i=1}^{N} \sum_{j=1}^{K} \gamma_{j}^{i}\left(\log \pi_{j}+\log \phi\left(\mathbf{x}^{i} ; \Theta_{j}\right)\right)
\end{aligned}
$$

which yields the same equation than 2.51 but substituting the latent variable $z_{j}^{i}$ by its posterior quantity $\gamma_{j}^{i}$. Setting derivatives of this expression obviously gives the same results as $2.53,2.55$ and 2.57 , but replacing $z_{j}^{i}$ by $\gamma_{j}^{i}$.

This formulation hence suggest an iterative scheme for MLE or MAP estimate, based on alternating between computing $p(Z \mid X ; \tilde{\Theta})$, referred as the Expectation-step or $E$-step; and updating the parameters of the model based on this distribution, referred as the Maximization-step or $M$-step. Therefore, the EM algorithm can be finally summarized as:

Initialization: Choose an initial setting for $\Theta^{(0)}$.

Expectation step: Estimate $p\left(Z \mid X ; \Theta^{(t)}\right)$

$$
p\left(z_{j}^{i}=1 \mid \mathbf{x}^{i} ; \Theta^{(t)}\right)=\gamma_{j}^{i(t)}=\frac{\pi_{j}^{(t)} \phi\left(\mathbf{x}^{i} ; \Theta_{j}^{(t)}\right)}{\sum_{k} \pi_{k}^{(t)} \phi\left(\mathbf{x}^{i} ; \Theta_{k}^{(t)}\right)}
$$

Maximization step: Update the parameters of the model given $p\left(Z \mid X ; \Theta^{(t)}\right)$

$$
\hat{\Theta}_{M L E}^{(t+1)}=\underset{\Theta}{\arg \max } \mathbb{E}_{p\left(Z \mid X ; \Theta^{(t)}\right)} \log \mathcal{L}(\Theta ; X, Z)
$$


where considering the GMM of the example:

$$
\begin{aligned}
\pi_{j}^{(t+1)} & =\frac{\sum_{i=1}^{N} \gamma_{j}^{i(t)}}{\sum_{i=1}^{N} \sum_{k=1}^{K} \gamma_{k}^{i(t)}} \\
\mu_{j}^{(t+1)} & =\frac{1}{\sum_{i=1}^{N} \gamma_{j}^{i(t)}} \sum_{i=1}^{N} \gamma_{j}^{i(t)} \mathbf{x}^{i} \\
\Sigma_{j}^{(t+1)} & =\frac{1}{\sum_{i=1}^{N} \gamma_{j}^{i(t)}} \sum_{i=1}^{N} \gamma_{j}^{i(t)}\left(\mathbf{x}^{i}-\mu_{j}^{(t+1)}\right)\left(\mathbf{x}^{i}-\mu_{j}^{(t+1)}\right)^{T}
\end{aligned}
$$

Convergence: Stop if $\mathcal{L}\left(\Theta^{(t+1)} ; X\right)-\mathcal{L}\left(\Theta^{(t)} ; X\right) \leq \epsilon$; otherwise $t=t+1$ and go to Expectation step.

\subsubsection{Spatially Varying Finite Mixture Model}

So far, we have seen that FMMs provide a rigorous statistical framework for modeling heterogeneous data in a precise and formal manner. The optimization of the likelihood function of a FMM provides a computationally feasible yet powerful solution to fit and evaluate complex models to model random variables.

However, FMMs make an important assumption that can turn into a severe drawback when it comes to structured data, such as images. FMMs assume observations $X$ to be independent and identically distributed, hence implying no correlations between them. This assumption does not hold for imaging data in which observations, i.e. pixels in $2 \mathrm{D}$ images or voxels in $3 \mathrm{D}$ volumes, are strictly arranged in a structure that inherently defines explicit correlations between them. Therefore, ignoring the prior knowledge that adjacent observations are more likely to belong to the same class wastes a highly useful information that allows images to be described in a more concise, adequate and realistic manner.

The Spatially Varying Finite Mixture Model (SVFMM) is an extension of the FMM for structured data, that aims to facilitate the modeling of the spatial correlations inherent in images. The SVFMM mainly differs from the FMM in the definition of the mixing coefficients which, as we saw, can also be interpreted as the prior probabilities of the components of the mixture. Specifically, the SVFMM assumes that each observation $\mathbf{x}^{i}$ has its own different vector of mixing coefficients $\pi^{i}$, denoted as contextual mixing coefficients, rather than sharing a global vector for all observations. Additionally, the SVFMM assumes $\Pi=\left(\pi^{1}, \ldots, \pi^{N}\right)$ to be a vector of random variables rather than parameters, allowing a prior density to be defined to introduce statistical correlations among them.

Let $X=\left(\mathbf{x}^{1}, \ldots, \mathbf{x}^{N}\right)$ a set of observations of a random variable, where $\mathbf{x}^{i} \in \mathbb{R}^{d}$. A SVFMM of $K$ components assumes a pdf over $X$ in the form: 


$$
p(X \mid \Theta, \Pi)=\prod_{i=1}^{N} \sum_{j=1}^{K} \pi_{j}^{i} \phi\left(\mathbf{x}^{i} ; \Theta_{j}\right)
$$

where $\Theta=\left\{\Theta_{1}, \ldots, \Theta_{K}\right\}$ and $\Pi=\left\{\pi^{1}, \ldots, \pi^{N}\right\}$ are the parameters of the model. Note that $\Theta$ and $\Pi$ are actually treated as random variables, and that each observation $\mathbf{x}^{i}$ has its own associated vector of contextual mixing coefficients $\pi^{i}$. Likewise FMM, contextual mixing coefficients must also satisfy $0 \leq \pi_{j}^{i} \leq 1$ and:

$$
\sum_{j=1}^{K} \pi_{j}^{i}=1
$$

A MAP estimate of the model is usually employed to introduce a proper density over $\Pi$, typically in the form of a MRF, to establish dependencies between adjacent contextual mixing coefficients. Therefore, the MAP estimate of the SVFMM is defined as:

$$
\begin{aligned}
(\hat{\Theta}, \hat{\Pi})_{M A P} & =\underset{(\Theta, \Pi)}{\arg \max } \log \mathcal{L}(X \mid \Theta, \Pi) \\
& =\underset{(\Theta, \Pi)}{\arg \max } \log p(\Theta, \Pi \mid X) \\
& =\underset{(\Theta, \Pi)}{\arg \max } \log \left\{\frac{p(X \mid \Theta, \Pi) p(\Theta, \Pi)}{p(X)}\right\} \\
& =\underset{(\Theta, \Pi)}{\arg \max } \log \{p(X \mid \Theta, \Pi) p(\Theta, \Pi)\} \\
& =\underset{(\Theta, \Pi)}{\arg \max } \log p(X \mid \Theta, \Pi)+\log p(\Theta, \Pi) \\
& =\underset{(\Theta, \Pi)}{\arg \max } \log p(X \mid \Theta, \Pi)+\log p(\Theta)+\log p(\Pi) \\
& =\underset{(\Theta, \Pi)}{\arg \max } \log p(X \mid \Theta, \Pi)+\log p(\Pi)
\end{aligned}
$$

where we have assumed independence in $p(\Theta, \Pi)$ and also assumed a constant uniform distribution for $p(\Theta)$ that gets rid out from the maximization.

Several densities for $p(\Pi)$ has been proposed in the literature (Sanjay-Gopal and Hebert, 1998; Woolrich et al, 2005; Blekas et al, 2005; Sfikas et al, 2008) to codify the concept that neighboring observations tend to share the same component of the mixture. A family of densities that has been widely used with successful results are the Gauss-MRFs (Nikou et al, 2007). This family of priors encodes the general idea that the estimator of the contextual mixing coefficients can be defined as the average of its spatial neighbors:

$$
\hat{\pi}^{i}=\frac{1}{\left|\mathcal{M}^{i}\right|} \sum_{m \in \mathcal{M}^{i}} \pi^{m}
$$


where $\mathcal{M}^{i}$ indicates the set of neighbors of the $i^{\text {th }}$ observation. In other words, this prior assumes that differences between adjacent contextual mixing coefficients for a given component $j$ of the mixture are Gaussian distributed in the form:

$$
\pi_{j}^{i}-\pi_{j}^{m} \sim \mathcal{N}\left(0, \beta^{2}\right) \quad m \in \mathcal{M}^{i}
$$

This approach is somewhat naïve and can be refined to capture variability among different components of the mixture and spatial directions of the images. The Directional Class-Adaptive Gauss-Markov Random Field (DCAGMRF) prior, which lies into the family of Simultaneous Auto-Regressive (SAR) models, has been used to regularize ill-posed inverse problems with successful results. The DCAGMRF takes the form:

$$
p(\Pi)=\prod_{i=1}^{N} \prod_{j=1}^{K} \prod_{d=1}^{D} \prod_{m \in \mathcal{M}_{d}^{i}} \frac{1}{\sqrt{2 \pi \beta_{j, d}^{2}}} \exp \left(-\frac{\left(\pi_{j}^{i}-\pi_{j}^{m}\right)^{2}}{2 \beta_{j, d}^{2}}\right)
$$

where sub-index $d$ refers to the different spatial adjacency directions (i.e. horizontals, verticals or diagonals), and $\mathcal{M}_{d}^{i}$ indicates the set of neighbors of the $i^{\text {th }}$ observation that lies in the $d^{\text {th }}$ spatial direction.

Merging 2.62 with 2.60 finally yields:

$$
(\hat{\Theta}, \hat{\Pi})_{M A P}=\underset{(\Theta, \Pi)}{\arg \max } \sum_{i=1}^{N} \log \sum_{j=1}^{K} \pi_{j}^{i} \phi\left(\mathbf{x}^{i} \mid \Theta_{j}\right)+\log p(\Pi)
$$

which again is analytically intractable, so numerical approximations should be employed.

\section{EM inference for SVFMM}

As in the FMM case, a binary $K$-dimensional one-hot encoding latent variable $Z$ is introduced to simplify the estimation of the model, by assuming knowledge about the component of the mixture to which the observation belongs to. The formulation of the SVFMM assuming the existence of the latent variables results in:

$$
p(X, Z \mid \Theta, \Pi)=\prod_{i=1}^{N} \prod_{j=1}^{K}\left(\pi_{j}^{i} \phi\left(\mathbf{x}^{i} ; \Theta_{j}\right)\right)^{z_{j}^{i}}
$$

The MAP estimate of the augmented SVFMM can be expressed as: 


$$
\begin{aligned}
(\hat{\Theta}, \hat{\Pi})_{M A P} & =\underset{(\Theta, \Pi)}{\arg \max } \log \mathcal{L}(X, Z \mid \Theta, \Pi) \\
& =\underset{(\Theta, \Pi)}{\arg \max } \log p(\Theta, \Pi \mid X, Z) \\
& \cdots \\
& =\underset{(\Theta, \Pi)}{\arg \max } \log p(X, Z \mid \Theta, \Pi)+\log p(\Pi) \\
& =\underset{(\Theta, \Pi)}{\arg \max } \log \prod_{i=1}^{N} \prod_{j=1}^{K}\left(\pi_{j}^{i} \phi\left(\mathbf{x}^{i} ; \Theta_{j}\right)\right)^{z_{j}^{i}}+\log p(\Pi) \\
& \cdots \quad \\
& =\underset{(\Theta, \Pi)}{\arg \max } \sum_{i=1}^{N} \sum_{j=1}^{K} z_{j}^{i}\left(\log \pi_{j}^{i}+\log \phi\left(\mathbf{x}^{i} ; \Theta_{j}\right)\right)+\log p(\Pi)
\end{aligned}
$$

where again is far easier to optimize than 2.66 and from where we can quickly observe that the maximization of $\Theta$ will be exactly the same as in the classic FMM. Only the estimation of $\pi_{j}^{i}$ will be affected by the density $p(\Pi)$ as expected.

Following the same reasoning than in classic FMM, the only information we can get from the latent variable $Z$ is given by its posterior density $p(Z \mid X, \tilde{\Theta}, \tilde{\Pi})$. Therefore, the conditional expectation of $\log \mathcal{L}(X, Z \mid \Theta, \Pi)$ under this density is used as auxiliar function for the optimization. Rearranging 2.65 into 2.68 , substituting $z_{j}^{i}$ by their posterior quantity $\gamma_{j}^{i}$ and dropping constant terms that do not affect the maximization yields the following $Q$-function:

$$
\mathcal{Q}(\Theta, \Pi \mid \tilde{\Theta}, \tilde{\Pi})=\sum_{i=1}^{N} \sum_{j=1}^{K} \gamma_{j}^{i}\left(\log \pi_{j}^{i}+\log \phi\left(\mathbf{x}^{i} ; \Theta_{j}\right)\right)-\sum_{d=1}^{D} \log \frac{\beta_{j, d}^{2}}{2}+\frac{\sum_{m \in \mathcal{M}_{d}^{i}}\left(\pi_{j}^{i}-\pi_{j}^{m}\right)^{2}}{2 \beta_{j, d}^{2}}
$$

Optimizing $\pi_{j}^{i}$ from expression 2.69 must take into account several considerations. First, note that $\pi_{j}^{i}$ appears in the term $\sum_{m \in \mathcal{M}_{d}^{i}}\left(\pi_{j}^{i}-\pi_{j}^{m}\right)^{2}$ once as the central individual and $D \cdot\left|\mathcal{M}_{d}^{i}\right|$ times as the neighbor of $\pi_{j}^{m}$ individuals. Second, no closed-form solution can be obtained when introducing the constraint $\sum_{j=1}^{K} \pi_{j}^{i}=1$ in the maximization. Instead, reparatory methods such as the quadratic programming algorithm proposed in (Blekas et al, 2005), must be employed to project the solutions onto the constraints to ensure probabilities sum up to 1 .

Taking all this into consideration, the updates for $\pi_{j}^{i}$ are obtained as the roots of the following second degree equation obtained by setting partial derivatives of 2.69 with respect $\pi_{j}^{i}$ (without restrictions):

$$
\left(\pi_{j}^{i}\right)^{2} \sum_{d=1}^{D} \frac{\left|\mathcal{M}_{d}^{i}\right|}{\beta_{j, d}^{2}}-\left(\pi_{j}^{i}\right) \sum_{d=1}^{D} \frac{\sum_{m \in \mathcal{M}_{d}^{i}} \pi_{j}^{m}}{\beta_{j, d}^{2}}-\frac{\gamma_{j}^{i}}{2}=0
$$


which can easily demonstrated that always have a real non negative solution.

The DCAGMRF prior also introduces a new set of parameters $\beta_{j, d}^{2}$ that govern the variances of the Gauss-MRF. Setting derivatives of 2.69 with respect $\beta_{j, d}^{2}$ yields:

$$
\beta_{j, d}^{2}=\frac{1}{N} \sum_{i=1}^{N} \sum_{m \in \mathcal{M}_{d}^{i}} \frac{\left(\pi_{j}^{i}-\pi_{j}^{m}\right)^{2}}{\left|\mathcal{M}_{d}^{i}\right|}
$$

Therefore, the MAP EM algorithm for the SVFMM with the DCAGMRF prior can be finally summarized as:

Initialization: Choose an initial setting for $\Theta^{(0)}$ and $\Pi^{(0)}$.

Expectation step: Estimate $p\left(Z \mid X, \Theta^{(t)}, \Pi^{(t)}\right)$

$$
p\left(z_{j}^{i}=1 \mid \mathbf{x}^{i}, \Theta^{(t)}, \Pi^{(t)}\right)=\gamma_{j}^{i(t)}=\frac{\pi_{j}^{i(t)} \phi\left(\mathbf{x}^{i} ; \Theta_{j}^{(t)}\right)}{\sum_{k} \pi_{k}^{i(t)} \phi\left(\mathbf{x}^{i} ; \Theta_{k}^{(t)}\right)}
$$

Maximization step: Update the parameters of the model given $p\left(Z \mid X, \Theta^{(t)} \Pi^{(t)}\right)$

$$
(\hat{\Theta}, \hat{\Pi})_{M A P}^{(t+1)}=\underset{(\Theta, \Pi)}{\arg \max } \mathbb{E}_{p\left(Z \mid X, \Theta^{(t)}, \Pi^{(t)}\right)} \log \mathcal{L}(X, Z \mid \Theta, \Pi)
$$

where considering the GMM example:

$$
\begin{aligned}
\left(\pi_{j}^{i(t+1)}\right)^{2} \sum_{d=1}^{D} \frac{\left|\mathcal{M}_{d}^{i}\right|}{\beta_{j, d}^{2}(t)}-\left(\pi_{j}^{i(t+1)}\right) \sum_{d=1}^{D} \frac{\sum_{m \in \mathcal{M}_{d}^{i}} \pi_{j}^{m(t)}}{\beta_{j, d}^{2}{ }^{(t)}}-\frac{\gamma_{j}^{i(t)}}{2}=0 \\
\mu_{j}^{(t+1)}=\frac{1}{\sum_{i=1}^{N} \gamma_{j}^{i(t)}} \sum_{i=1}^{N} \gamma_{j}^{i(t)} \mathbf{x}^{i} \\
\Sigma_{j}^{(t+1)}=\frac{1}{\sum_{i=1}^{N} \gamma_{j}^{i(t)}} \sum_{i=1}^{N} \gamma_{j}^{i(t)}\left(\mathbf{x}^{i}-\mu_{j}^{(t+1)}\right)\left(\mathbf{x}^{i}-\mu_{j}^{(t+1)}\right)^{T} \\
\beta_{j, d}^{2(t+1)}=\frac{1}{N} \sum_{i=1}^{N} \sum_{m \in \mathcal{M}_{d}^{i}} \frac{\left(\pi_{j}^{i(t+1)}-\pi_{j}^{m(t+1)}\right)^{2}}{\left|\mathcal{M}_{d}^{i}\right|}
\end{aligned}
$$

(*) Choose the real non-negative solution and project onto the constraints using the quadratic programming algorithm (Blekas et al, 2005) to ensure $\sum_{j=1}^{K} \pi_{j}^{i}=1$ 
Convergence: Stop if $\mathcal{L}\left(X \mid \Theta^{(t+1)}, \Pi^{(t+1)}\right)-\mathcal{L}\left(X \mid \Theta^{(t)}, \Pi^{(t)}\right) \leq \epsilon$; otherwise $t=$ $t+1$ and go to Expectation step.

\subsubsection{Dirichlet Compound Multinomial-Spatially Varying Fi- nite Mixture Model}

An important limitation of the SVFMM is that it does not inherently preserves the condition of $\sum_{j} \pi_{j}^{i}=1$. Instead, reparatory projections must be employed to accomplish with this constraint, thereby compromising the assumed Bayesian framework.

An interesting alternative is to consider that $\Pi$ follows a Dirichlet Compound Multinomial (DCM) distribution (Nikou et al, 2010). The DCM distribution is a hierarchical discrete multivariate distribution, where an observation $\mathbf{z}^{i}$ is drawn from a Multinomial distribution governed by a parameter vector $\pi^{i}$, which in turn is drawn from a Dirichlet distribution governed by parameter vector $\alpha^{i}$.

$$
\begin{aligned}
& z^{i} \sim \operatorname{Cat}_{K}\left(\pi^{i}\right) \\
& \pi^{i} \sim \operatorname{Dir}_{K}\left(\alpha^{i}\right)
\end{aligned}
$$

Such scheme presents two important advantages for the SVFMM. First, the Dirichlet distribution draws probability vectors that intrinsically satisfies the constraint $\sum_{j} \pi_{j}^{i}=1$, so no reparatory corrections will be required. Next, the $\alpha^{i}$ parameters of the Dirichlet distribution are not subject to any restriction (rather than $\alpha_{j}^{i} \geq 0$ ) so a Gauss-MRF can be imposed over them to introduce the spatial correlations and local regularity in a more straightforward manner.

In this sense, the pdf of the $\mathbf{z}^{i}$ variable given the parameters of the Categorical distribution (Multinomial unit-length with $n=1$ ) is:

$$
p\left(\mathbf{z}^{i} \mid \pi^{i}\right)=\frac{n !}{\prod_{j=1}^{K}\left(z_{j}^{i}\right) !} \prod_{j=1}^{K}\left(\pi_{j}^{i}\right)^{z_{j}^{i}}=\prod_{j=1}^{K}\left(\pi_{j}^{i}\right)^{z_{j}^{i}}
$$

The pdf of the $\pi^{i}$ variable given the parameters of the Dirichlet distribution is

$$
p\left(\pi^{i} \mid \alpha^{i}\right)=\frac{\Gamma\left(\sum_{j=1}^{K} \alpha_{j}^{i}\right)}{\prod_{j=1}^{K} \Gamma\left(\alpha_{j}^{i}\right)} \prod_{j=1}^{K} \pi_{j}^{i\left(\alpha_{j}^{i}-1\right)}
$$

with $\Gamma(\cdot)$ the Gamma function. Integrating out both pdfs we obtain the following density for the $\mathbf{z}^{i}$ variables conditioned to $\alpha^{i}$ :

$$
p\left(\mathbf{z}^{i} \mid \alpha^{i}\right)=\frac{\Gamma\left(\sum_{j=1}^{K} \alpha_{j}^{i}\right)}{\Gamma\left(\sum_{j=1}^{K} \alpha_{j}^{i}+z_{j}^{i}\right)} \prod_{j=1}^{K} \frac{\Gamma\left(\alpha_{j}^{i}+z_{j}^{i}\right)}{\Gamma\left(\alpha_{j}^{i}\right)}
$$


Taking into account that $\Gamma(x+1)=x \Gamma(x)$, the vector $\pi^{i}$ of label prior probabilities can be finally computed as:

$$
\pi_{j}^{i}=\frac{\alpha_{j}^{i}}{\sum_{j=1}^{K} \alpha_{k}^{i}}
$$

Therefore, the previously proposed DCAGMRF of equation 2.65 can be imposed over the parameters of the Dirichlet distribution in the form:

$$
p(A)=\prod_{i=1}^{N} \prod_{j=1}^{K} \prod_{d=1}^{D} \prod_{m \in \mathcal{M}_{d}^{i}} \frac{1}{\sqrt{2 \pi \beta_{j, d}^{2}}} \exp \left(-\frac{\left(\alpha_{j}^{i}-\alpha_{j}^{m}\right)^{2}}{2 \beta_{j, d}^{2}}\right)
$$

Arranging this density into the SVFMM and substituting $\pi^{i}$ by its conditional density under the Dirichlet process yields:

$$
(\hat{\Theta}, \hat{A})_{M A P}=\underset{(\Theta, \Pi)}{\arg \max } \sum_{i=1}^{N} \log \sum_{j=1}^{K} \frac{\alpha_{j}^{i}}{\sum_{k=1}^{K} \alpha_{k}^{i}} \phi\left(\mathbf{x}^{i} \mid \Theta_{j}\right)+\log p(A)
$$

which, as expected, is again analytically intractable.

\section{EM inference for DCM-SVFMM}

As in the previous cases, a binary $K$-dimensional one-hot encoding latent variable $Z$ is introduced to simplify the estimation of the model. The formulation of the DCMSVFMM assuming the existence of this latent variable results in:

$$
p(X, Z \mid \Theta, A)=\prod_{i=1}^{N} \prod_{j=1}^{K}\left(\frac{\alpha_{j}^{i}}{\sum_{k=1}^{K} \alpha_{k}^{i}} \phi\left(\mathbf{x}^{i} ; \Theta_{j}\right)\right)^{z_{j}^{i}}
$$

The MAP estimate of the augmented DCM-SVFMM is expressed as: 


$$
\begin{aligned}
(\hat{\Theta}, \hat{A})_{M A P} & =\underset{(\Theta, A)}{\arg \max } \log \mathcal{L}(X, Z \mid \Theta, \Pi) \\
& =\underset{(\Theta, A)}{\arg \max } \log p(\Theta, A \mid X, Z) \\
& \cdots \\
& =\underset{(\Theta, A)}{\arg \max } \log p(X, Z \mid \Theta, A)+\log p(A) \\
& =\underset{(\Theta, A)}{\arg \max } \log \prod_{i=1}^{N} \prod_{j=1}^{K}\left(\frac{\alpha_{j}^{i}}{\sum_{k=1}^{K} \alpha_{k}^{i}}\left(\mathbf{x}^{i} ; \Theta_{j}\right)\right)^{z_{j}^{i}}+\log p(A) \\
& \ldots \quad)_{(\Theta, A)} \sum_{i=1}^{N} \sum_{j=1}^{K} z_{j}^{i}\left(\log \frac{\alpha_{j}^{i}}{\sum_{k=1}^{K} \alpha_{k}^{i}}+\log \phi\left(\mathbf{x}^{i} ; \Theta_{j}\right)\right)+\log p(A) \\
& =\underset{\arg \max }{\arg })^{K}
\end{aligned}
$$

which, as usual, is easier to optimize than the original model.

The conditional expectation of $\log \mathcal{L}(X, Z \mid \Theta, A)$ under the posterior density $p(Z \mid X, \tilde{\Theta}, \tilde{A})$ is again used as auxiliar function for the optimization, yielding the following $Q$ function:

$$
\mathcal{Q}(\Theta, A \mid \tilde{\Theta}, \tilde{A})=\sum_{i=1}^{N} \sum_{j=1}^{K} \gamma_{j}^{i}\left(\log \frac{\alpha_{j}^{i}}{\sum_{k=1}^{K} \alpha_{k}^{i}}+\log \phi\left(\mathbf{x}^{i} ; \Theta_{j}\right)\right)-\sum_{d=1}^{D} \log \frac{\beta_{j, d}^{2}}{2}+\frac{\sum_{m \in \mathcal{M}_{d}^{i}}\left(\alpha_{j}^{i}-\alpha_{j}^{m}\right)^{2}}{2 \beta_{j, d}^{2}}
$$

As in the SVFMM case, the optimization of $\Theta$ is exactly equal than in classic FMMs. The optimization of $\alpha_{j}^{i}$, however, does not require to introduce any constraint to preserve the condition $\sum_{j} \pi_{j}^{i}=1$. Then, it is only necessary to consider that $\alpha_{j}^{i}$ appears in the term $\sum_{m \in \mathcal{M}_{d}^{i}}\left(\alpha_{j}^{i}-\alpha_{j}^{m}\right)^{2}$ once as the central individual and $D \cdot\left|\mathcal{M}_{d}^{i}\right|$ times as the neighbor of $\alpha_{j}^{m}$ individuals.

Setting partial derivatives of 2.81 with respect $\alpha_{j}^{i}$ yields the following third degree equation:

$$
\left(\alpha_{j}^{i}\right)^{3}+\left(\alpha_{j}^{i}\right)^{2}\left(A_{-j}^{i}-\frac{C_{j}^{i}}{B_{j}^{i}}\right)-\left(\alpha_{j}^{i}\right)\left(\frac{A_{-j}^{i} C_{j}^{i}}{B_{j}^{i}}\right)-\frac{z_{j}^{i} A_{-j}^{i}}{2 B_{j}^{i}}=0
$$

where 


$$
\begin{aligned}
A_{-j}^{i} & =\sum_{\substack{k=1 \\
k \neq j}}^{K} \alpha_{k}^{i} \\
B_{j}^{i} & =\sum_{d=1}^{D} \frac{\left|\mathcal{M}_{d}^{i}\right|}{\beta_{j, d}^{2}} \\
C_{j}^{i} & =\sum_{d=1}^{D} \frac{\sum_{m \in \mathcal{M}_{d}^{i}} \alpha_{j}^{m}}{\beta_{j, d}^{2}}
\end{aligned}
$$

where, under polynomial theory, it can be demonstrated that there is always a real non negative solution that satisfies $\alpha_{j}^{i} \geq 0$. The Cardano's or Vieta's methods can be employed to algebraically obtain the roots of the proposed third degree equation.

The optimization of $\beta_{j, d}^{2}$ yields the same equation than 2.71 but expressed in terms of $\alpha$ variables:

$$
\beta_{j, d}^{2}=\frac{1}{N} \sum_{i=1}^{N} \sum_{m \in \mathcal{M}_{d}^{i}} \frac{\left(\alpha_{j}^{i}-\alpha_{j}^{m}\right)^{2}}{\left|\mathcal{M}_{d}^{i}\right|}
$$

Therefore, the MAP EM algorithm for the DCM-SVFMM with the DCAGMRF prior can be finally summarized as:

Initialization: Choose an initial setting for $\Theta^{(0)}$ and $A^{(0)}$.

Expectation step: Estimate $p\left(Z \mid X, \Theta^{(t)}, A^{(t)}\right)$

$$
p\left(z_{j}^{i}=1 \mid \mathbf{x}^{i}, \Theta^{(t)}, A^{(t)}\right)=\gamma_{j}^{i(t)}=\frac{\pi_{j}^{i(t)} \phi\left(\mathbf{x}^{i} ; \Theta_{j}^{(t)}\right)}{\sum_{k} \pi_{k}^{i(t)} \phi\left(\mathbf{x}^{i} ; \Theta_{k}^{(t)}\right)}
$$

where

$$
\pi_{j}^{i(t)}=\frac{\alpha_{j}^{i^{(t)}}}{\sum_{j=1}^{K} \alpha_{k}^{i^{(t)}}}
$$

Maximization step: Update the parameters of the model given $p\left(Z \mid X, \Theta^{(t)} A^{(t)}\right)$

$$
(\hat{\Theta}, \hat{A})_{M A P}^{(t+1)}=\underset{(\Theta, A)}{\arg \max } \mathbb{E}_{p\left(Z \mid X, \Theta^{(t)}, A^{(t)}\right)} \log \mathcal{L}(X, Z \mid \Theta, A)
$$

where considering the GMM example:

$$
\left(\alpha_{j}^{i(t+1)}\right)^{3}+\left(\alpha_{j}^{i(t+1)}\right)^{2}\left(A_{-j}^{i}{ }^{(t)}-\frac{C_{j}^{i(t)}}{B_{j}^{i(t)}}\right)-\left(\alpha_{j}^{i(t+1)}\right)\left(\frac{A_{-j}^{i}{ }^{(t)} C_{j}^{i(t)}}{B_{j}^{i(t)}}\right)-\frac{z_{j}^{i} A_{-j}^{i}(t)}{2 B_{j}^{i(t)}}=0 \quad(*)
$$




$$
\begin{aligned}
\mu_{j}^{(t+1)} & =\frac{1}{\sum_{i=1}^{N} \gamma_{j}^{i(t)}} \sum_{i=1}^{N} \gamma_{j}^{i(t)} \mathbf{x}^{i} \\
\Sigma_{j}^{(t+1)} & =\frac{1}{\sum_{i=1}^{N} \gamma_{j}^{i(t)}} \sum_{i=1}^{N} \gamma_{j}^{i(t)}\left(\mathbf{x}^{i}-\mu_{j}^{(t+1)}\right)\left(\mathbf{x}^{i}-\mu_{j}^{(t+1)}\right)^{T} \\
\beta_{j, d}^{2}{ }^{(t+1)} & =\frac{1}{N} \sum_{i=1}^{N} \sum_{m \in \mathcal{M}_{d}^{i}} \frac{\left(\alpha_{j}^{i(t+1)}-\alpha_{j}^{m(t+1)}\right)^{2}}{\left|\mathcal{M}_{d}^{i}\right|}
\end{aligned}
$$

(*) Choose the real non-negative solution.

Convergence: Stop if $\mathcal{L}\left(X \mid \Theta^{(t+1)}, A^{(t+1)}\right)-\mathcal{L}\left(X \mid \Theta^{(t)}, A^{(t)}\right) \leq \epsilon$; otherwise $t=$ $t+1$ and go to Expectation step.

\subsection{Deep Learning}

At the beginning of the 2000's the ML started an important revolution with the birth of the Deep Learning (DL) techniques (LeCun et al, 2015). Until that time, ML was generally constituted by two disjoint and clearly differentiated stages: the feature extraction step and the classification/regression step. The feature extraction step was typically addressed manually, computing hand-crafted features from the raw data guided by the expertise, knowledge and intuition of the researcher. These features were then fed to the classifier/regressor, expecting to be discriminant informational inputs to successfully solve the corresponding task. Therefore, a crucial step for the good performance of classic ML methods typically relied in a powerful feature extraction process.

The qualitative leap made by DL techniques concerns mainly the feature extraction stage. Instead of computing hand-crafted features, DL models attempt to simultaneously learn a discriminant representation of the raw input data at the same time as the classifier (regressor) is trained. This concept shift allows machines to learn the optimal set of features appropriate for the task. Therefore, DL models not only learn the decision boundaries (or regression model) of a discriminative classifier, but they also learn a manifold to optimally project the data into it in such a way that the classification task becomes as trivial as possible: the so-called representation learning. Figure 2.12 depicts a general conceptual schema of the concept shift between classic ML approach and the current DL paradigm.

DL addresses the representation learning problem by building concepts out of simpler concepts. This kind of hierarchical learning perfectly fits into the nature of Artificial Neural Networks (ANNs). ANNs are ML models that process the input hierarchically by passing it through a nested set of layers, each of which learns a deeper level of abstraction of the input. Therefore, concepts inside a ANN are built by concatenating the output of a layer, which represents a simpler abstract representation of the input, and the input of the successive layer, which learns a more complex and richer 


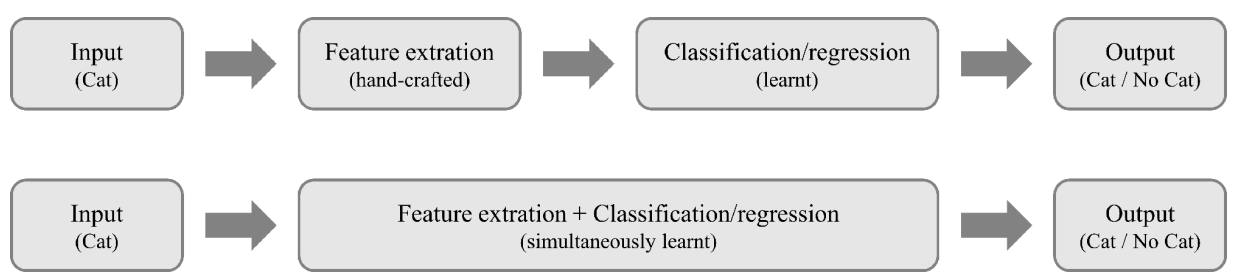

Figure 2.12: Conceptual schema of the differences between classic ML and DL.

composite representation from those previous outputs. Figure 2.13 shows an example of the hierarchical concept representation of images showing faces by a DL model.
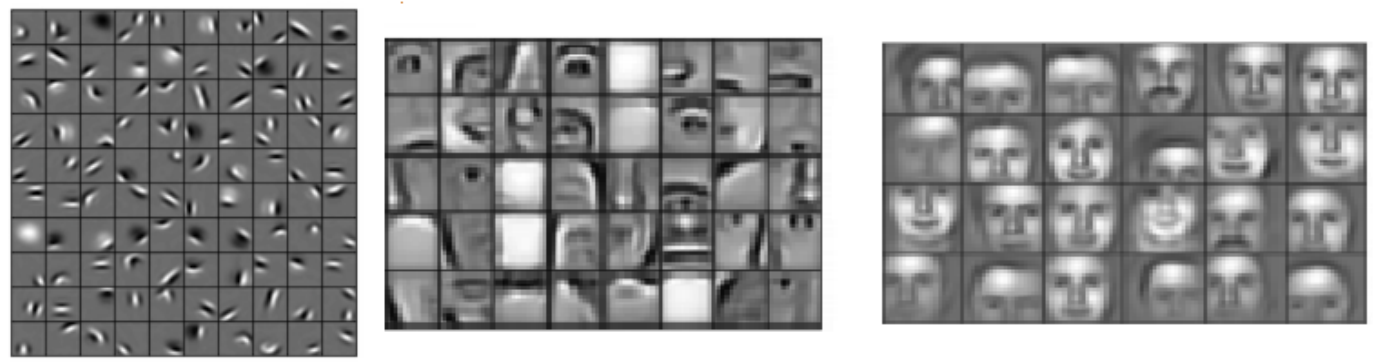

Figure 2.13: Example of hierarchical features extracted during the learning process of a DL model. Image taken with kind permission from https://devblogs.nvidia.com/ deep-learning-nutshell-core-concepts/hierarchical_features/

The following sections briefly describe the basics and fundamental principles of ANNs, and present the Convolutional Neural Networks (CNNs): the current state-ofthe-art classifier for most of the supervised visual computing related tasks.

\subsubsection{Artificial Neural Networks}

ANNs are connectionist computational models in which a number of processors, named artificial neurons or perceptrons are interconnected in a manner inspired by the connections between neurons in a human brain. ANNs were born in the mid-twentieth century, in a combined effort of multiple researchers. In 1943 McCulloch and Pitts (1943) created the first computational model of a biological neuron. Few years later Hebb (2002) designed the first learning rule for ANNs based on the concept of neural plasticity, whose premise was that if two neurons were active simultaneously, then the strength of the connection between them should increase. In 1958, Rosenblatt (1958) created the perceptron, which is the basis of the ANNs, and Ivakhnenko and Lapa (1967) developed the first multi-layer ANN. In subsequent years, Kelley (1960) laid the basis of the backpropagation algorithm, but it was not until the work of Rumelhart et al (1986) where the first ANN was effectively trained using the backpropagation algorithm by adjusting the weights of the network proportional to the gradient computed from the error.

Today, ANNs are the most powerful and widely used ML algorithms, as they have proven to largely outperform other approaches in many tasks such as visual object recognition, object detection and segmentation and many other domains such as speech 
recognition, handwritten text recognition or financial time series prediction. The following sections provides an overview of the basics of ANNs to facilitate the reading and understanding of subsequent chapters and contributions of this thesis.

\section{The Perceptron}

ANNs are composed by basic units called perceptrons or artificial neurons. The perceptron (see Figure 2.14), in its most elementary form, is a simplified model of a biological neuron, approximated as a mathematical function for learning a binary linear classifier. Let $\mathbf{x}=\left\{x_{1}, \ldots, x_{D}\right\}$ a $\mathbb{R}^{D}$ vector of features representing an observation. Mathematically, a perceptron is defined as:

$$
f(\mathbf{x})= \begin{cases}1 & \text { if } \sum_{i} w_{i} x_{i}+b>0 \\ 0 & \text { otherwise }\end{cases}
$$

where $w_{i}$ represents a weight associated to the $x_{i}$ feature, and $b$ stands for the bias of the linear model. The Heaviside step activation function is employed in the most basic perceptron algorithm, setting the output of the neuron to 1 ("activated") if $\sum_{i} w_{i} x_{i}+b$ is greater than a threshold (typically 0); or to 0 ("deactivated") otherwise.

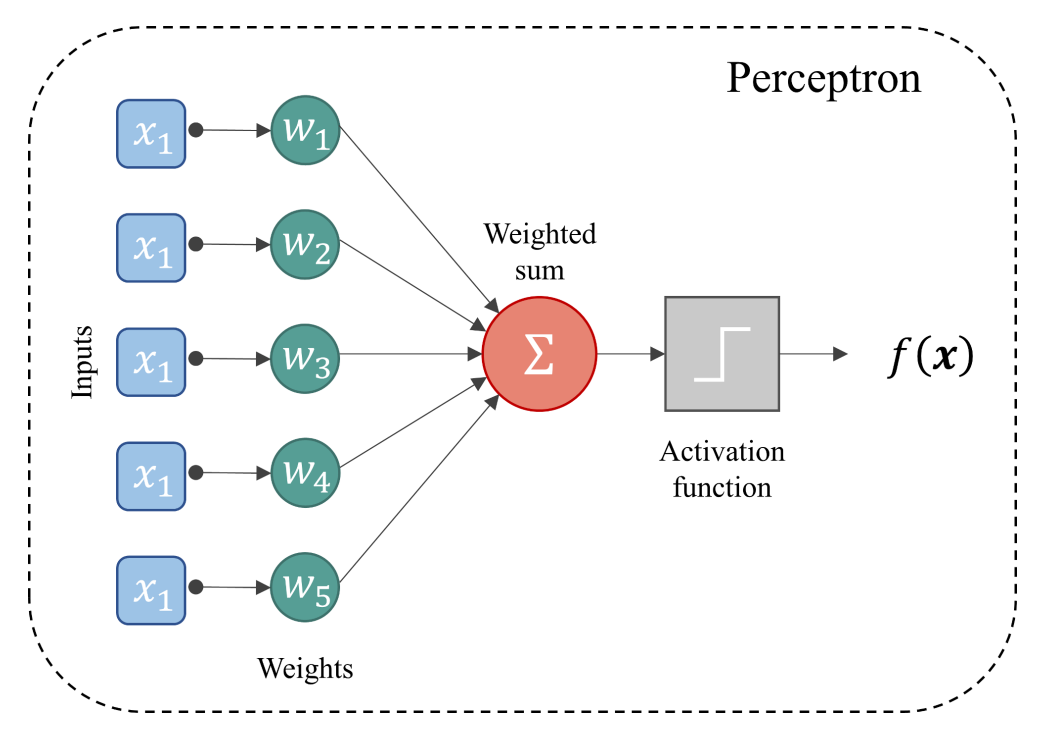

Figure 2.14: The perceptron.

Perceptrons are only able to solve linearly separable problems regardless of the activation function employed. Therefore, they are only guaranteed to converge if the training set is linearly separable. Otherwise, the perceptron will fail and no approximate solution is returned.

\section{The Multi-Layer Perceptron}

A Multi-Layer Perceptron (MLP) is a class of feedforward ANN composed of at least three layers of interconnected perceptrons. Figure 2.15 shows an example of a simple MLP with an input layer, a hidden layer and an output layer. Except of the input layer, all the neurons in the other layers are perceptrons. 


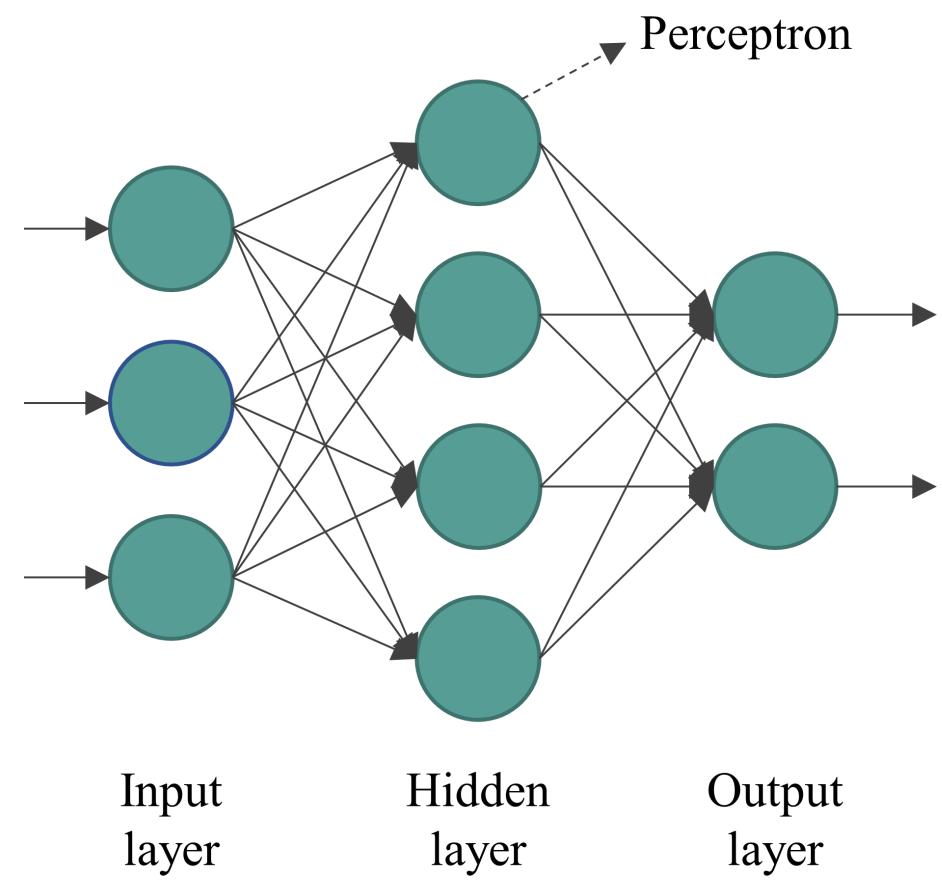

Figure 2.15: A multi-layer perceptron.

The arrangement of multiple layers of perceptrons in combination with the nonlinear activation functions allow MLPs to solve non-linearly separable problems. Indeed, it is demonstrated that MLPs are universal mathematical function approximators through superposition of non-linearly activated perceptrons.

\section{Activation functions}

Activation functions are determinant for ANNs since they are fundamental to allow the network to solve non-linear problems. An ANN of multiple layers, each one activated by a linear function passed to the next layer, results in a final function that is a combination of linear functions in a linear form, which by definition can be replaced by a single linear function. In other words, no matter how many layers are stacked in an ANN, if only linear activation functions are used, the network is still equivalent to a single layer with linear activation, and therefore is only capable of solving linear problems.

To mitigate this effect multitude of activation functions are proposed in the literature. The most important are summarized below:

Linear activation The most simple activation function. The output of the perceptron is proportional to the input. It is defined as:

$$
f(x)=c x
$$

It allows a continuous output range, which is more powerful than a binary output, however its derivative with respect to $x$ is constant. This means that the gradient has no relation to $x$ and, therefore, regardless of the magnitude of the prediction error, 
the changes made by backpropagation are constant. Additionally, as said above, a multi-layer ANN fully activated by linear functions is equivalent to a single-layer ANN only capable of solving linear problems.

Heaviside step activation A binary activation function. The output of the perceptron is "activated" if $\sum_{i} w_{i} x_{i}+b$ is greater than a threshold, or is "deactivated" otherwise. It is typically defined as:

$$
f(x)= \begin{cases}1 & \text { if } \sum_{i} w_{i} x_{i}+b>0 \\ 0 & \text { otherwise }\end{cases}
$$

where "activated" is typically represented by 1 , and "deactivated" fires the 0 value. Likewise, the typical threshold employed to determine the output is the 0 value.

The Heaviside function outputs a binary discrete value, which presents some disadvantages. By definition it can only properly represent binary classification problems, in which the ANN has only one output neuron. For multi-label problems where ANNs have multiple output neurons firing Heaviside step activations, it is not possible to correctly identify the corresponding class, hence preventing their training. Moreover, continuous activation functions allow a smoother training process, less prone to fall into bad local minima.

Sigmoid / Logistic activation One of the most historically used activation functions. It represents the continuous soft approximation of the Heaviside step function. It is mathematically defined as:

$$
f(x)=\frac{1}{1+\exp (-x)}
$$

Its non-linearity allows the network to learn complex decision boundaries by stacking layers sequentially. Moreover, the gradient is related to the input, so it propagates the magnitude of the error through the entire network. Finally, the output is continuous and bounded to the $[0,1]$ range, allowing a smoother training process without gradient explosion.

The main disadvantage of this activation function is that it originates the so-called gradient vanishing problem. The gradient vanishing problem is related to the update process of the $w_{i}$ weights of a network trained through gradient-based learning algorithms such as backpropagation. In these algorithms, the weights of the network are updated proportionally to the partial derivative of the error function with respect to the weights. Depending on the nature of the activation function, it is possible for the gradient to become extremely small, barely modifying the weights of the network, and therefore slowing or even preventing the network from further training.

In the specific case of the sigmoid activation function, it can be seen that for $x$ values above $x=2$ and below $x=-2$, the slope of the function is almost near-horizontal. Therefore, there is no effectively change in the gradient, resulting in the network refusing to learn further. For small networks, this does not represent a big problem, however, the more layers stacked in the network, the problem grows exponentially, eventually collapsing the training of the model. 
Tahn activation A similar activation function than the sigmoid function. Indeed, the tanh activation is a scaled version of the sigmoid function:

$$
f(x)=\tanh (x)=\frac{2}{1+\exp (-2 x)}-1=2 \operatorname{sigmoid}(2 x)-1
$$

It has similar properties than the sigmoid function. Its gradient is stronger than the sigmoid but it also suffers from the vanishing gradient problem.

Softmax activation Typically an activation function only employed in the final layer of a network to convert the activations into probabilities (posteriors). It is defined as:

$$
f(\mathbf{x})=\frac{\exp \left(x_{i}\right)}{\sum_{j} \exp \left(x_{j}\right)}
$$

The aim of this activation function is to normalize the outputs so that each neuron triggers a value in the range $[0,1]$ and all of them add up to 1 , giving the probability of the input value being in a specific class.

ReLU activation First presented in 2009, the Rectified Linear Unit (ReLU) activation function can be considered a milestone in DL. It is mathematically defined as:

$$
f(x)=\max (0, x)
$$

This simple function provides a set of benefits that have made ReLU as the most widely used activation function nowadays. First, ReLU is a non-linear function, so it allows the network to model complex functions by stacking layers. Moreover, the function is ranged between $[0,+\infty)$, hence allowing the gradient to not vanish as there is no saturation in any range of the function. It also provides sparsity activation since multiple neurons can fire 0 activation because of a negative input of the ReLU, therefore becoming a lighter network.

However, ReLU activation suffers from the so-called dying ReLU problem. This problem arises when a neuron continuously trigger negative values to the ReLU. In these cases, the gradient become 0 , preventing the neuron from responding to changes in error, and updating its weights. If the problem affects multiple neurons in the network, it could lead to a substantial part of the network passive.

Leaky-ReLU activation It consist of a simple modification of the ReLU function to avoid the dying ReLU problem:

$$
f(x)=\max (\alpha x, x)
$$

with $\alpha$ a parameter, typically adopting small values $(\alpha=0.01)$, to allow a small positive slope for the negative range of the function. Such modification allows the neuron to prevent its paralysis, eventually reactivating it during the training process. 


\section{Backpropagation algorithm}

The backpropagation algorithm was originally introduced in the 1970s, but its important was not fully recognized until the 1986 paper by Rumelhart et al (1986). Backpropagation is an algorithm to efficiently train an ANN by adjusting its weights so that the network output minimizes a given loss function. More formally, the backpropagation is an iterative optimization algorithm based on a gradient descent technique, to update the weights of a network by computing the gradient of the loss function with respect to each weight following the chain rule.

To illustrate the mechanics of the backpropagation algorithm, lets consider the simple ANN shown in figure 2.16 (example taken from https://www.anotsorandomwalk. com/backpropagation-example-with-numbers-step-by-step/ with kind permission).

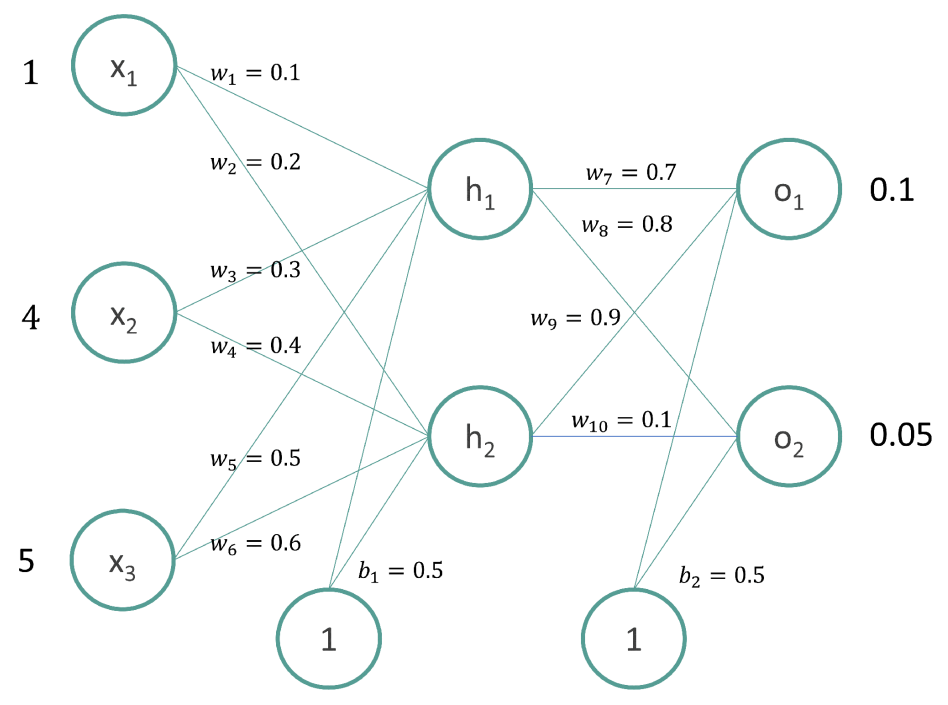

Figure 2.16: ANN example.

The following relations are established between the neurons of the network:

$$
\begin{aligned}
& y_{h_{1}}=w_{1} x_{1}+w_{3} x_{2}+w_{5} x_{3}+b_{1} \\
& y_{h_{2}}=w_{2} x_{1}+w_{4} x_{2}+w_{6} x_{3}+b_{1} \\
& z_{h_{1}}=f\left(y_{1 h_{1}}\right) \\
& z_{h_{2}}=f\left(y_{h_{2}}\right) \\
& y_{o_{1}}=w_{7} z_{h_{1}}+w_{9} z_{h_{2}}+b_{2} \\
& y_{o_{2}}=w_{8} z_{h_{1}}+w_{10} z_{h_{2}}+b_{2} \\
& z_{o_{1}}=\sigma\left(y_{o_{1}}\right) \\
& z_{o_{2}}=\sigma\left(y_{o_{2}}\right)
\end{aligned}
$$

where $y$. refers to the input of the corresponding neuron, and $z$. indicates the output of the neuron after the activation function $f(y)$ is applied, which in the example will be the sigmoid function. 
Let assume the input values of the network $x_{1}=1, x_{2}=4, x_{3}=5$ and the desired target $t_{1}=0.1, t_{2}=0.05$. The forward pass of the network yields the following results:

$$
\begin{aligned}
& y_{h_{1}}=0.1(1)+0.3(4)+0.5(5)+0.5=4.3 \\
& y_{h_{2}}=0.2(1)+0.4(4)+0.6(5)+0.5=5.3 \\
& z_{h_{1}}=\sigma(4.3)=0.9866 \\
& z_{h_{2}}=\sigma(5.4)=0.9950 \\
& y_{o_{1}}=0.7(0.9866)+0.9(0.9950)+0.5=2.0862 \\
& y_{o_{2}}=0.8(0.9866)+0.1(0.9950)+0.5=1.3888 \\
& z_{o_{1}}=\sigma(2.0862)=0.8896 \\
& z_{o_{2}}=\sigma(1.3888)=0.8004
\end{aligned}
$$

Once the forward pass is completed, weights are updated by setting partial derivatives of the error function $E$ with respect to each weight, following the chain rule. As an example, setting partial derivatives to update the weight $w_{7}$ yield:

$$
\frac{\partial E}{\partial w_{7}}=\frac{\partial E}{\partial z_{o_{1}}} \cdot \frac{\partial z_{o_{1}}}{\partial y_{o_{1}}} \cdot \frac{\partial y_{o_{1}}}{\partial w_{7}}
$$

For the shake of simplicity lets consider $E$ the sum of squared errors of the predictions with respect to the targets:

$$
E=\frac{1}{2}\left[\left(z_{o_{1}}-t_{1}\right)^{2}+\left(z_{o_{2}}-t_{2}\right)^{2}\right]
$$

Solving for the first term $\frac{\partial E}{\partial z_{o_{1}}}$ of the partial derivatives in equation 2.93 yields:

$$
\frac{\partial E}{\partial z_{o_{1}}}=z_{o_{1}}-t_{1}=0.8896-0.1=0.7896
$$

The next term to solve is $\frac{\partial z_{o_{1}}}{\partial y_{o_{1}}}$, which only engages the activation function of the neuron. The activation function chosen for the example is the sigmoid function, whose derivative is $x(1-x)$. Applying to the update process of the weights yields:

$$
\frac{\partial z_{o_{1}}}{\partial y_{o_{1}}}=z_{o_{1}}\left(1-z_{o_{1}}\right)=0.8896(1-0.8896)=0.0982
$$

Finally, the partial derivative $\frac{\partial y_{o_{1}}}{\partial w_{7}}$ involves the derivation of $w_{7} z_{h_{1}}+w_{9} z_{h_{2}}+b_{2}$ with respect $w_{7}$, which is trivial:

$$
\frac{\partial y_{o_{1}}}{\partial w_{7}}=z_{h_{1}}=0.9866
$$

Therefore, the gradient of the error with respect to the weight $w_{7}$ is:

$$
\frac{\partial E}{\partial w_{7}}=0.7896 \cdot 0.0982 \cdot 0.9866=0.0765
$$


Once the gradient of the loss function with respect to the corresponding weight is known, the final update of the weight is typically performed by:

$$
\hat{w}_{7}=w_{7}-\eta \frac{\partial E}{\partial w_{7}}=0.7-(0.01 \cdot 0.0765)=0.6992
$$

with $\eta$ referring to the learning rate typically set to small values $(\eta=0.01)$.

The updates of the remaining weights follow the same mechanics, with a slightly more cumbersome computation due to the increase in the number of neurons interconnected in deeper layers. The results are:

$$
\begin{array}{ll}
\hat{w}_{1}=w_{1}-\eta \frac{\partial E}{\partial w_{1}}=0.1000 & \hat{w}_{7}=w_{7}-\eta \frac{\partial E}{\partial w_{7}}=0.6992 \\
\hat{w}_{2}=w_{2}-\eta \frac{\partial E}{\partial w_{2}}=0.2000 & \hat{w}_{8}=w_{8}-\eta \frac{\partial E}{\partial w_{8}}=0.7988 \\
\hat{w}_{3}=w_{3}-\eta \frac{\partial E}{\partial w_{3}}=0.2999 & \hat{w}_{9}=w_{9}-\eta \frac{\partial E}{\partial w_{9}}=0.8992 \\
\hat{w}_{4}=w_{4}-\eta \frac{\partial E}{\partial w_{4}}=0.4000 & \hat{w}_{10}=w_{10}-\eta \frac{\partial E}{\partial w_{10}}=0.0988 \\
\hat{w}_{5}=w_{5}-\eta \frac{\partial E}{\partial w_{5}}=0.4999 & \hat{b}_{1}=b_{1}-\eta \frac{\partial E}{\partial b_{1}}=0.5000 \\
\hat{w}_{6}=w_{6}-\eta \frac{\partial E}{\partial w_{6}}=0.6000 & \hat{b}_{2}=b_{2}-\eta \frac{\partial E}{\partial b_{2}}=0.4980
\end{array}
$$

After weight updating, computing the output of the network in the iteration $t=1$ yields:

$$
\begin{aligned}
& z_{o_{1}}^{(1)}=0.8892 \\
& z_{o_{2}}^{(1)}=0.7997
\end{aligned}
$$

Comparing the errors produced by the network in the first $(t=0)$ and second iterations $(t=1)$ :

$$
\begin{aligned}
& E^{(0)}=0.5933 \\
& E^{(1)}=0.5925
\end{aligned}
$$

which initially may not seem too much, but after repeating the process 100.000 times, the error decreases to 0.0000351085 , and the output of the network becomes:

$$
\begin{array}{lll}
z_{o_{1}}^{(100 k)}=0.1175 & \rightarrow \quad\left(\text { vs. } t_{1}=0.1\right) \\
z_{o_{1}}^{(100 k)}=0.0582 & \rightarrow \quad\left(\text { vs. } t_{2}=0.05\right)
\end{array}
$$

concluding the training of the network. For an in-depth dissertation on ANNs, training procedures, numerical optimizers and convergence properties of DL models please refer to Bengio (2017). 


\subsubsection{Convolutional Neural Networks}

CNNs are a specialized type of ANNs inspired in the human visual cortex. In the 1950s, experiments conducted by Dr. Hubel and Dr. Wiesel identified that sets of different specific neurons in a cat's visual cortex responded very quickly when observing images with lines at specific angles, with light and dark patterns, or by observing movement in a certain direction. These results laid the foundation for further research into animal's visual understanding.

Human visual cortex process the information by passing it from one cortical area to another, where each cortical area is more specialized than the last one. For example, the primary visual cortex focuses on preserving spatial location of visual information, i.e. orientation of edges and lines. The secondary visual cortex feeds on the response of the primary visual cortex and focuses on collecting spatial frequencies, size, colors and shape of the object, while third visual cortex process the global motion and provides a complete visual representation. CNNs adopts this philosophy and tries to act like a computational visual cortex.

CNNs are a type of ANN mainly oriented for image processing, that arose in the attempt to solve the limitations that classic MLPs have when dealing with images. First, fully-connected ANNs do not scale well for images. Consider the example of a small image of $100 \times 100$ pixels. Each neuron of the second layer of a fully-connected ANN has 10000 weights to learn. Therefore, assuming a simple MLP with a hidden layer of 1000 neurons has $10 \mathrm{M}$ of weights only in the second layer, making it unaffordable to optimize. Second, MLP also ignore the spatial information and local redundancy inherent in images. In a MLP, the pixels of an image are independently connected to the layers of the network, thus loosing the semantic meaning of the position and its relation with their neighbors. This wastes a highly useful information that allows images to be described in a more concise, adequate and realistic manner. Finally, classic MLPs are not translation-invariant. Since the input image is connected in a static manner to the layers of the network, the patterns learned during the training process are statically linked to the position in which they appeared. Therefore, same objects appearing at different positions will change the weights associated to the corresponding pixels, intrinsically learning the position in which they appeared.

CNNs, by the opposite, are non-fully connected ANNs, primarily made of a set of stacked convolution layers, where each layer has a small set of shared weights that intelligently adapt to the properties of images. They are by definition translation invariant and context-sensitive, allowing for learning patterns related to objects of interest appearing anywhere in the image.

The following sections overall introduce the main components and state-of-the-art architectures for image segmentation with CNNs.

\section{Convolution layer}

A convolution layer is a special arrangement of neurons, typically in a 2D or 3D grid, that acts as a bank of learnable filters (sometimes also called kernels). These filters are used to perform a sliding dot product with an image to extract the set of optimal features to solve the classification/regression task. The dot product operation, also 
named convolution, is defined as:

$$
I \otimes K=\sum_{i} I_{i} \cdot K_{i}
$$

where $I$ and $K$ are arrays of the same dimension, and $i$ represents the index to traverse such arrays. Figure 2.17 shows an example of a convolution between a kernel of size $3 \times 3$ and a region of an image of the same size.

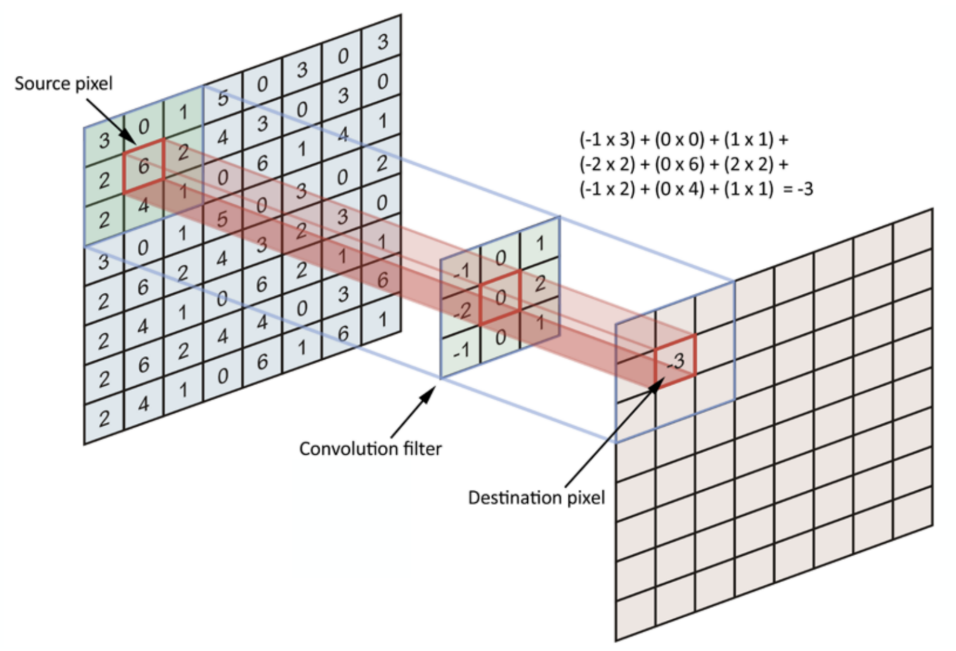

Figure 2.17: The convolution operation. Image taken with kind permission from https:// towardsdatascience.com/simple-introduction-to-convolutional-neural-networks-cdf8d3077bac.

Therefore, unlike the classic fully-connected layers of an ANN, a convolution layer is not fully connected to its input, but it contains small banks of filters of shared weights that traverse the different locations of the image. This introduces several benefits: first, the number of weights to train in a CNN is drastically lower than on a classic ANN. Considering the previous aforementioned example, a CNN with a first layer of 100 learnable filters of size $3 \times 3$ has only 900 weights to train, regardless of the image size. Moreover, the $3 \times 3$ weights of each filter are shared across the entire image, making the pattern learned by the filter invariant to location and translation. Finally, the 2D or 3D arrangement of the filter weights inherently captures the spatial information and the semantic meaning of the positions in which the intensities appear in the images.

In addition to the kernel size, convolution operation also involves two parameters that must be taken into account: the stride and the padding methods. The stride controls how the filter convolves around the input image. In other words, the stride fixes how many pixels the kernel is moved in one direction between two successive convolutions. Therefore, a stride of 1 means that, after a convolution, the kernel must be moved one pixel in only one direction and then perform the next convolution. The padding controls the behavior of the convolution in terms of the size of the resulting map after the convolution. The convolution operation, by definition, shrinks the image into a factor related to the size of the kernel used for the operation. Additionally, pixels in the corners and edges of the images are not visited the same number of times as central pixels, thus, giving more importance to the latter. To compensate for this effect, 
a padding method can be employed to pad the input image so that the resulting map after the convolution has the same size than the input image. There are different types of padding: zero-padding, mirror-padding and reflect-padding; with zero-padding the most widely used in CNNs.

\section{Batch Normalization layer}

Batch normalization is a technique employed to normalize the inputs of a layer, with the main purpose of mitigating the internal covariate shift problem. The covariate shift problem refers to the continuous shift that the internal activations of the network undergo during the training process, due to continuous changes in the distribution of the inputs of the layers. Since the inputs of a layer are the activations of the previous layer, a significant change in the distribution of these activations forces the intermediate layers to continuously adapt itself to these numerical fluctuations. This leads to a situation where each layer in the ANN wastes training iterations in this adaptation rather than learning the relations between the inputs and the desired target.

The batch normalization technique prevents this situation by normalizing the activations of a layer, before feeding them as the input of the next layer. Let $X=$ $\left(\mathbf{x}^{1}, \ldots, \mathbf{x}^{N}\right)$ a batch of $N$ inputs of a layer, the batch normalization performs as follows:

$$
\begin{aligned}
\mu_{\mathcal{B}} & =\frac{1}{N} \sum_{i=1}^{N} \mathbf{x}^{i} \\
\sigma_{\mathcal{B}}^{2} & =\frac{1}{N} \sum_{i=1}^{N}\left(\mathbf{x}^{i}-\mu_{\mathcal{B}}\right)^{2} \\
\hat{\mathbf{x}}^{i} & =\gamma \frac{\mathbf{x}^{i}-\mu_{\mathcal{B}}}{\sqrt{\sigma_{\mathcal{B}}^{2}}}+\beta
\end{aligned}
$$

where $\mu_{\mathcal{B}}$ is the mean of the batch, $\sigma_{\mathcal{B}}^{2}$ is the variance of the batch and $\hat{\mathbf{x}}^{i}$ is the $i^{\text {th }}$ normalized sample of the batch. Batch normalization technique introduces two learnable parameters, $\gamma$ and $\beta$, to allow the network to perform a scale and shift of the batch if the training process requires it.

Besides mitigating the internal covariate shift, batch normalization also provides other related benefits to the training process of an ANN. First, batch normalization allows each layer to learn a little bit more independently of other layers. Since the inputs are always normalized in the same numerical range, the layer can focus on learning the relations between the inputs rather than the relations between the adjacent layers. Second, batch normalization also significantly accelerates the training process. Higher learning rates can be employed when using batch normalization since it ensures that there are no a outlier activations. Finally, it reduces overfitting because it has a slight regularization effect over the entire network. 


\section{Pooling layer}

The function of the pooling layers is to progressively reduce the size of the activation maps in order to also reduce the number of weights and the computational cost of the network, while retaining the most discriminant information. Additionally, pooling also allows to increase the receptive field of the network by compressing the information in smaller activation maps.

There are three main types of pooling layers: max pooling, average pooling and sum pooling. Max pooling - the most frequently pooling layer used in CNNs - uses filters to take the largest elements of the activation maps in the pooling window. Figure 2.18 shows an example of the result of a max pooling layer with filters of $2 \times 2$ and stride 2 .

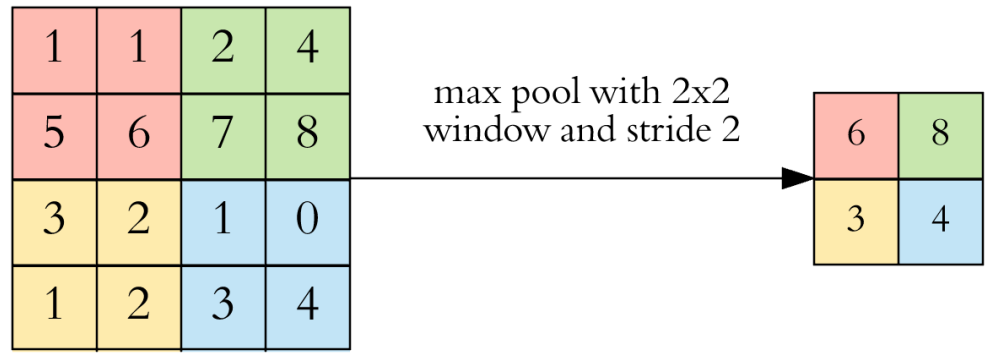

Figure 2.18: Max pooling with filters of size $2 \times 2$ and stride 2 . Image taken with kind permission from https://towardsdatascience.com/ applied-deep-learning-part-4-convolutional-neural-networks-584bc134c1e2.

As expected, average pooling computes the average value within the pooling layer, while sum pooling computes the summation of the values inside each pooling layer.

Pooling operation also provides benefits for preventing overfitting. In a general sense, pooling is a feature selection method by reducing the dimensions of input. Therefore, selecting a subset of activations from the output of the layers drop useless information and introduce slight variations in the new activation maps that helps in overfitting to specific patterns.

\section{U-Net architecture}

The U-Net architecture was first proposed by Ronneberger et al (2015) as a modification of the classic Fully convolutional network presented in (Long et al, 2015). It consist of a contracting path similar than the fully convolutional network, followed by and expanding path where pooling layers are replaced by up-sampling layers. This gives the architecture a "U" shape that justifies its name.

The main innovation of this architecture was the expanding path made up of upsampling convolutions that allowed to reconstruct the low-dimensional activation maps into high-dimensional maps of the same size of input image. This enabled to address image segmentation problems through CNNs in a complete naturally manner, since the output activation maps could directly represent the logits of each class for each pixel of the image. 
Figure 2.19 shows the original architecture proposed in the article of Ronneberger et al (2015). The network consisted in 5 levels of depth, with 64 activation maps in the first level, 128 in the second, 256 in the third, 512 in the fourth and 1024 in the final level. The contracting path was formed by blocks of two sequential $3 \times 3$ convolutions layers followed by max pooling layers with filters of size $2 \times 2$ at each level. The expanding path performed the opposite job by gradually projecting the lowdimensional activation maps into the space of the inputs, so that the final activation map had as many channels as the number of classes in which each pixel could be classified.

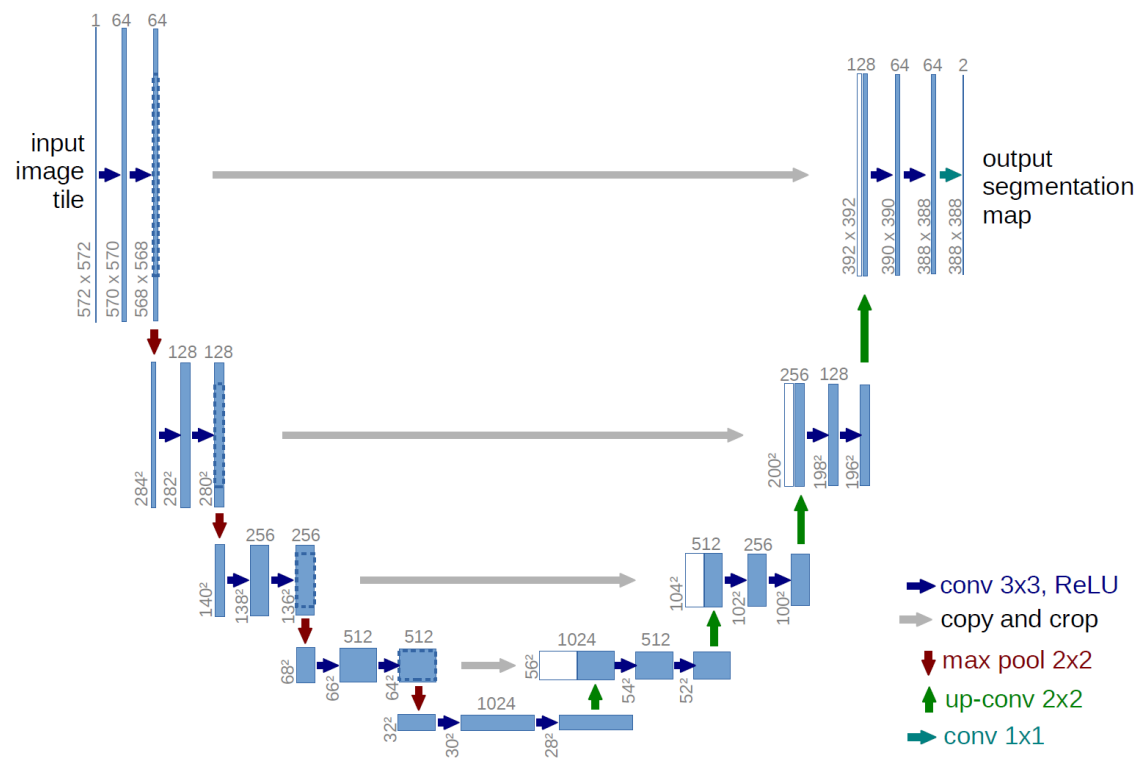

Figure 2.19: U-Net original architecture.

Another important contribution was the loss employed to train the network. The work of Ronneberger et al (2015) was the first that proposed to apply a pixel-wise softmax to the final activation map, in order to convert the activations into probabilities that represent the degree of confidence of each pixel to belong to each class. Following this approach, the network was finally trained by minimizing the cross-entropy loss function between the softmax predictions and the groundthruth segmentation.

The final significant contribution of the paper was the so-called long-term skip connections between same levels in the contracting and expanding paths of the U-Net architecture (denoted as gray arrows in the figure 2.19). These connections are also biologically inspired in the human visual cortex, where neurons of the first areas of the cortex are directly connected with neurons in deeper regions, skipping intermediary cortex areas. This allows to directly propagate useful signals between different regions of the cortex to properly understand the scene. The same mechanism is mimicked in U-Net architectures with the long-term skip connections. These connections introduce two main benefits in the network: first, they allow to easily propagate the gradient to the first layers, where it is more difficult to adjust weights (due to gradient vanishing problem). Second, these connections bring the ability to directly pass useful information captured in the first layers that is later required to reconstruct the activation 
maps during the up-sampling.

Since 2012, as a result of the breakthrough brought about by AlexNet (Krizhevsky et al, 2012), CNNs, and specifically U-Net architectures, clearly dominate most of the computer vision and image understanding challenges, positioning them as the de facto standard DL classifiers for addressing these tasks. 
Chapter 2. Rationale 


\section{Chapter 3}

\section{Comparative study of unsupervised learning algorithms for glioblastoma segmentation}

Unsupervised learning constitutes one of the most important roles in automated image understanding. Specifically, it has historically played a key role in the medical image segmentation task, as it provided the first approaches to identify tissues in MRI acquisitions without requiring manual human intervention. However, currently, medical image segmentation is often dominated by supervised learning methods because of their superior performance over unsupervised learning methods. Nevertheless, supervised learning approaches have several limitations. The performance of supervised learning models is directly conditioned by the size and quality of the training corpus, whose collection is often tedious, time-consuming and sometimes even unaffordable. Though, most importantly, supervised learning can only learn tasks for which humans already know the solution. Because of their learning scheme, supervised approaches are only able to solve problems with well-known defined outputs. Unsupervised learning, on the contrary, is capable of recognizing patterns within the data in completely unexplored and unknown tasks. Regarding the image segmentation problem, unsupervised learning is able to delineate regions within the image with common MRI properties, typically representing the same tissue or physiological area. However, due to the unguided blind learning approach of unsupervised methods, these generally do not reach as accurate results as those of a supervised learning model in well-known tasks.

In this sense, the aim of this chapter is to demonstrate that unsupervised learning can achieve competitive results comparable to those obtained by supervised learning methods in a well-known task. The purpose is to ratify if unsupervised learning is capable to detect consistent patterns in MRI, discriminant enough to solve a segmentation task. For that purpose, we performed a comparative study of several unsupervised learning algorithms in the task of automated high grade glioma segmentation. A postprocessing stage was also developed to automatically map each label of an unsupervised segmentations to a specific tissue of the brain. The comparative was performed with the public reference BRAin Tumor Segmentation (BRATS) 2013 Test and Leaderboard datasets. 
Chapter 3. Comparative study of unsupervised learning algorithms for glioblastoma

segmentation

The contents of this chapter were published in the publications (Juan-Albarracin et al, 2015b,a)-thesis contributions C1, P1 and P2.

\subsection{Introduction}

Medical imaging plays an indisputable key role in the diagnosis and management of brain tumors. The intracranial location of these lesions and the unspecificity of clinical symptoms make medical imaging a necessary tool to diagnose and monitor the evolution of these diseases (Wen et al, 2010). Specifically, the early identification and delineation of the different tissues composing the tumor becomes crucial to take decisions that can improve the patient survival. However, the manual segmentation of these tissues constitutes a complex, time-consuming and biased task, which caught the attention of the ML community (Bauer et al, 2013). Particularly, glioblastoma tumor has received most of this attention, as it is the most common and aggressive malignant tumor of the central nervous system (Dolecek et al, 2012; Deimling, 2009). Glioblastomas are heterogeneous lesions that present different areas of active tumor, necrosis and edema, all of them exhibiting a high variability related to the aggressiveness of the tumor. Hence, the automated segmentation of these lesions becomes a desired solution from the clinical standpoint and an interesting challenge to address from the ML community.

Extensive reviews of brain tumor segmentation have been presented by Wadhwa et al (2019); Saman and Jamjala Narayanan (2019); Gordillo et al (2013); Bauer et al (2013). Most of these techniques fall into the supervised learning approach. Verma et al (2008) and Ruan et al (2011) employed Support Vector Machines (SVMs) to segment healthy and pathological tissues, and additionally subcompartiments inside these areas. Jensen and Schmainda (2009) used neural networks to detect brain tumor invasion. Lee et al (2008) used a combination of Conditional Random Field (CRF) and SVM to perform tumor segmentation. Bauer et al (2011) also used SVM and Hierarchical CRF to segment both healthy and tumor tissues including sub-compartments. Recently, Random Forest (RF) techniques have shown high success in the supervised brain tumor segmentation task. Meier et al (2013); Festa et al (2013); Reza and Iftekharuddin (2013); Tustison et al (2013) proposed several approaches based on RF variants for the BRATS challenge of Medical Image Computing and Computer Assisted Intervention (MICCAI) 2013 Conference, reaching the first positions in the competition. Nowadays, with the advent of novel deep learning techniques, the current state-of-the art is mostly dominated by CNN classifiers. CNNs are a class of deep feed-forward neural networks whose architecture is particularly well suited for computer vision recognition tasks. CNNs have outperformed most of algorithms in many medical image segmentation problems, arising as the winner technique in most challenges such as BRATS, ISLES or PROMISE12 challenges. Crimi et al $(2015,2016,2017,2018)$ summarize the most relevant contributions to the BRATS challenge from 2015 to 2018, demonstrating the superiority of CNNs in the glioma segmentation task.

However, supervised learning performance depends directly on the quality and size of the training dataset, which often requires an expensive, time-consuming and biased task to collect (Gordillo et al, 2013). Moreover, changes in MRI protocols, acquisition 
routines or clinical environments may distort the data and hence affect the performance of the supervised models (Duda et al, 2000). However, the major drawback of supervised learning is that it is only capable to address tasks that humans have already solved before. The mimic nature of supervised learning limits the paradigm to the set of problems with known solution, which, without underestimating its unquestionable usefulness, reduces the ML to an instrument for automatizing well-known tasks.

On the contrary, unsupervised learning address these limitations in a more straightforward manner. Unsupervised learning does not require a training corpus from which to learn a model to solve the task, but it analyzes unlabeled data searching for hidden patterns and inner relationships that describes their latent structure (Wittek, 2014). Therefore, besides solving already known tasks, this approach has the innate ability to discover new knowledge from the data by exploring the arrangement of the information. Sticking to the glioblastoma segmentation task, unsupervised learning typically fits a customized segmentation model to the patient's MRI, describing their own imaging patterns. This makes it possible to characterize the lesion in each case just by discriminating its own patterns and not by reinterpreting them under the knowledge drawn from other examples. By the opposite, the absence of manual segmentations to guide the learning process makes segmentation more challenging and often lead to a worse performance with respect to supervised approaches.

Some attempts for brain tissue segmentation have been made under the unsupervised paradigm. Fletcher-Heath et al (2001) proposed an approach based on fuzzy clustering and domain knowledge for multi-parametric non-enhancing tumor segmentation. Domain knowledge and parenchymal tissue detection was based on heuristics related to geometric shapes and lesion locations, which may not be robust when high deformation is presented. Moreover, several assumptions such as prior knowledge about the number of existing tumor foci or the minimum required thickness of the MRI slices introduced significant limitations to the method. Nie et al (2009) used Gaussian clustering with a spatial accuracy-weighted Hidden Markov Random Field (HMRF) that allowed them to deal with images at different resolutions without interpolation. Nowadays, advanced reconstruction techniques such as super-resolution enables to work in a high resolution voxel space by reconstructing the low resolution images without interpolation. Moreover, no automated method was provided to differentiate between tumor labels and normal tissue labels after the unsupervised segmentation ends. Zhu et al (2012) developed a software based on the segmentation approach proposed by Zhang et al (2001), which performs an GMM clustering combined whit HMRFs. Zhu et al extended Zhang's approach through a sequence of additionally morphological and thresholding operations to refine the segmentation. Such operations are not fully specified and only overall commented, so the reproducibility of their results is not possible. Vijayakumar et al (2007) proposed a method based on Self-Organizing Maps (SOMs) to segment tumor, necrosis, cysts, edema and normal tissues using MRI. Although the learning process of SOMs was performed in an unsupervised manner, the dataset from which to infer the net structure was determined manually, similar than in a supervised approach. In their work, 700 pattern observations, corresponding to 7 different tissues, where selected manually, hence converting the process in a supervised task. Prastawa et al (2003) proposed a similar approach than the followed in this study. They per- 
formed an unsupervised classification based on FMMs and also used a brain atlas to characterize the normal tissue labels. However, they made important simplifying assumptions to allow them to use the atlas without registration. Moreover, they also simplify the segmentation task in 2 labels (tumor and edema), ignoring other important tissues such as necrosis or non-enhancing tumor. Doyle et al (2013) also proposed an approach based on GMM clustering and MRF priors. They defined different penalizations in the MRF depending on the adjacency of the labels to prevent incoherent segmentations, however they did not clearly specify how they related the glioblastoma tissues with these labels before running the unsupervised segmentation. Furthermore, all the unsupervised approaches described above applied their algorithms on its own datasets, making difficult a general comparison of the methods.

In this work, we propose a fully automated pipeline for unsupervised glioblastoma segmentation. Our contributions concern the assessment of the performance of several unsupervised segmentation methods, including both structured and non-structured classification algorithms, on a real public and reference dataset; and we also provide a generalized method to automatically identify pathological labels in an unsupervised segmentation that represent abnormal tissues in the brain. Our aim is to demonstrate that unsupervised segmentation algorithms can achieve competitive results, comparable to supervised approaches, by detecting imaging patterns that describe the tissue's MRI profiles.

We evaluated our unsupervised segmentation method using the public BRATS 2013 Leaderboard and Test datasets provided for the International Image Segmentation Challenge of MICCAI Conference. The proposed method with the GMM algorithm improves the results obtained by most of the supervised approaches evaluated with the Leaderboard BRATS 2013 set, reaching the 2nd position in the rank. Our variant using the Gauss-HMRF improves the results obtained by the best unsupervised segmentation methods evaluated with the BRATS 2013 Test set, and also reaches the 7th position in the general Test rank, mainly against supervised approaches.

\subsection{Materials}

In order to make our results comparable, we have used the public multi-modal BRATS dataset 2013 (Menze et al, 2015), provided for the international BRATS 2013 challenge in image segmentation of MICCAI conference. We have evaluated our method with the Test set and the Leaderboard set, and we have made a comparison between our proposed method and the best algorithms that participated in the challenge.

The BRATS 2013 Test set consists of multi-contrast MR scans of 10 high-grade glioma patients without the manual expert labeling. The Leaderboard set consists of $11+10$ multi-contrast MR scans of high-grade glioma patients, also without the manual expert labeling. The first 11 Leaderboard patients come from to the Test set of BRATS 2012 Challenge, while the next 10 cases refer to the new Leaderboard cases for 2013 Challenge.

For each patient of the datasets, $\mathrm{T}_{1}$-weighted, $\mathrm{T}_{2}$-weighted, FLAIR and postgadolinium $\mathrm{T}_{1 C E}$-weighted $\mathrm{MR}$ images were provided. All images were linearly coregistered to the post-gadolinium $\mathrm{T}_{1 C E}$-weighted sequence, skull stripped, and inter- 
polated to $1 \mathrm{~mm}^{3}$ isotropic resolution. No inter-patient registration was made to put all the images in a common reference space.

Expert manual annotations of the MRI studies were performed, considering five possible labels for the lesion:

Label 0: background, brain and everything else not corresponding to labels 1, 2, 3 and 4.

Label 1: non-brain, non-tumor, necrosis, cyst and hemorrhage.

Label 2: surrounding edema.

Label 3: non-enhancing tumor.

Label 4: enhancing tumor.

An evaluation web page was provided to assess the quality of the segmentations, computing different metrics such as Dice coefficient, positive predictive value, sensitivity and Kappa indices. Furthermore, the evaluation was made over different subcompartments of the lesion, to properly measure the performance of the different segmentation methods. The set of labels composing each sub-compartment are described in section 3.3.5.

\subsection{Methods}

This section describes the proposed pipeline for the automated unsupervised segmentation of glioblastoma, including a generalized postprocessing designed to identify the pathological classes of an unsupervised segmentation that represent abnormal tissues in the brain.

\subsubsection{MRI preprocessing}

MRI preprocessing is an active field of research that attempts to enhance and correct $\mathrm{MR}$ images for their posterior analysis. In an unsupervised segmentation approach there is no reference nor manual labeling from which to learn tumor tissue models. Therefore, common artifacts such as noise, NMR inhomogeneities or registration missalignments can introduce undesired patterns into images that could led to erroneous classifications, describing MRI artifacts rather than physiological tissues. This clearly increases the importance of an accurate and effective preprocess of the MRI if an unsupervised segmentation is going to be performed. We propose the following scheme for the BRATS 2013 data: 1) Denoising, 2) Skull stripping, 3) Bias field correction and 4) Super-resolution.

\section{Denoising}

Denoising is a standard MRI preprocessing task that aims to reduce or ideally remove the noise from an MR image. Although MRI noise has been usually modeled as Gaussian distributed, by definition MRI noise follows a Rician distribution (Gudbjartsson 
and Patz, 1995). Diaz et al (2011) presented a comprehensive analysis of different denoising methods, discussing their weaknesses and strengths. Recent filters such as the NLM introduced by Buades et al (2005b) has improved the existing techniques for MR data. Based on this approach, Manjón et al (2010c) introduced a variant of the NLM filter, called Adaptive-NLM filter, which does not assume an uniform distribution of the noise over the image, thereby adapting the strength of the filter depending on a local estimation of the noise. The filter also deals with both correlated Gaussian and Rician noise. We used the Adaptive-NLMfilter to remove the noise from the BRATS images.

\section{Brain extraction}

Brain extraction, also called skull-stripping, comprises the process of removing skull, extra-meningeal and non-brain tissues from the MRI sequences. Although BRATS 2013 dataset is already skull stripped, we detected several cases that include partial areas of the cranium and extra-meningeal tissues. In order to improve the preprocessing of the data, we recomputed the skull stripping masks for all patients using the Brain Suite Software, removing the non desired tissues of the MR images. Figure 3.1 shows an example of a patient of the BRATS 2013 dataset with the original skull stripping, the resultant image after our new skull stripping and the remaining residual.
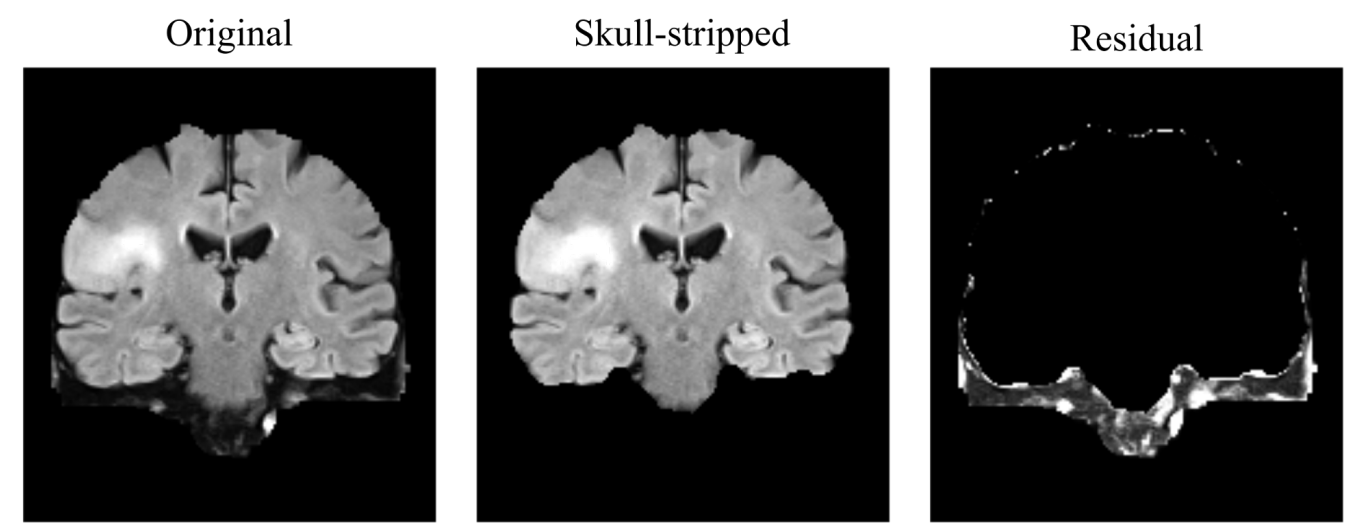

Figure 3.1: Example of the proposed skull stripping. From left to right column: original BRATS 2013 patient image, resultant image after the proposed skull stripping and the remaining residual.

\section{Bias field correction}

Intensity inhomogeneity is another common artifact present in MRI acquisitions. Magnetic field inhomogeneities are unavoidable effects consisting on low frequency signals that corrupt the images and affect their intensity levels. Typically, automated segmentation approaches based on MRI are built upon the assumption that tissues have the same distribution of intensity across the image. Therefore, intensity inhomogeneities must be corrected to ensure a correct coherent segmentation. The popular non-parametric non-uniform intensity normalization N3 algorithm was proposed in 1998 by Sled et al (1998), becoming a reference technique for bias field correcting. 
Tustison et al (2010) proposed in 2010 a new implementation of N3, called N4, which improves the N3 algorithm with a better B-spline fitting function and a hierarchical optimization scheme for the bias field correction. N4 was used in our study to correct MRI inhomogeneities.

\section{Super-resolution}

In a brain tumor lesion protocol, several MR sequences are commonly acquired normally at different resolutions, thereby introducing spatial inconsistencies when a multimodal MR study is performed. In these cases, an upsampling or interpolation is needed to set a common voxel space for all sequences. Classic interpolation such as linear, cubic or splines interpolation could rise as a solution, but at the cost of introducing artifacts such as partial volume effects or stair-case artifacts. In contrast, more powerful and sophisticated methods such as super resolution could improve classic interpolation by reconstructing the low resolution images recovering its high frequency components. Several super resolution schemes for MRI are available in the literature (Plenge et al, 2013; Manjón et al, 2010a; Rousseau and Alzheimer's Disease Neuroimaging Initiative, 2010; Protter et al, 2009).

BRATS 2013 dataset comes with a $1 \mathrm{~mm}^{3}$ isotropic voxel size resolution achieved through classic interpolation. In order to improve the resolution of these images, we employed the super resolution algorithm proposed by Manjón et al (2010b), which exploits the self-similarity present in MR images through a patch-based non-local reconstruction process. Such method iteratively reconstructs a high resolution image by applying a NLM filter with different strengths, aimed to increase image regularity while constraining intensity ranges so that they be coherent among scales through a local back-projection approach. Figure 3.2 shows an example of a super resolved FLAIR sequence of a patient of the BRATS 2013 dataset with a detailed zoom of an axial slice.
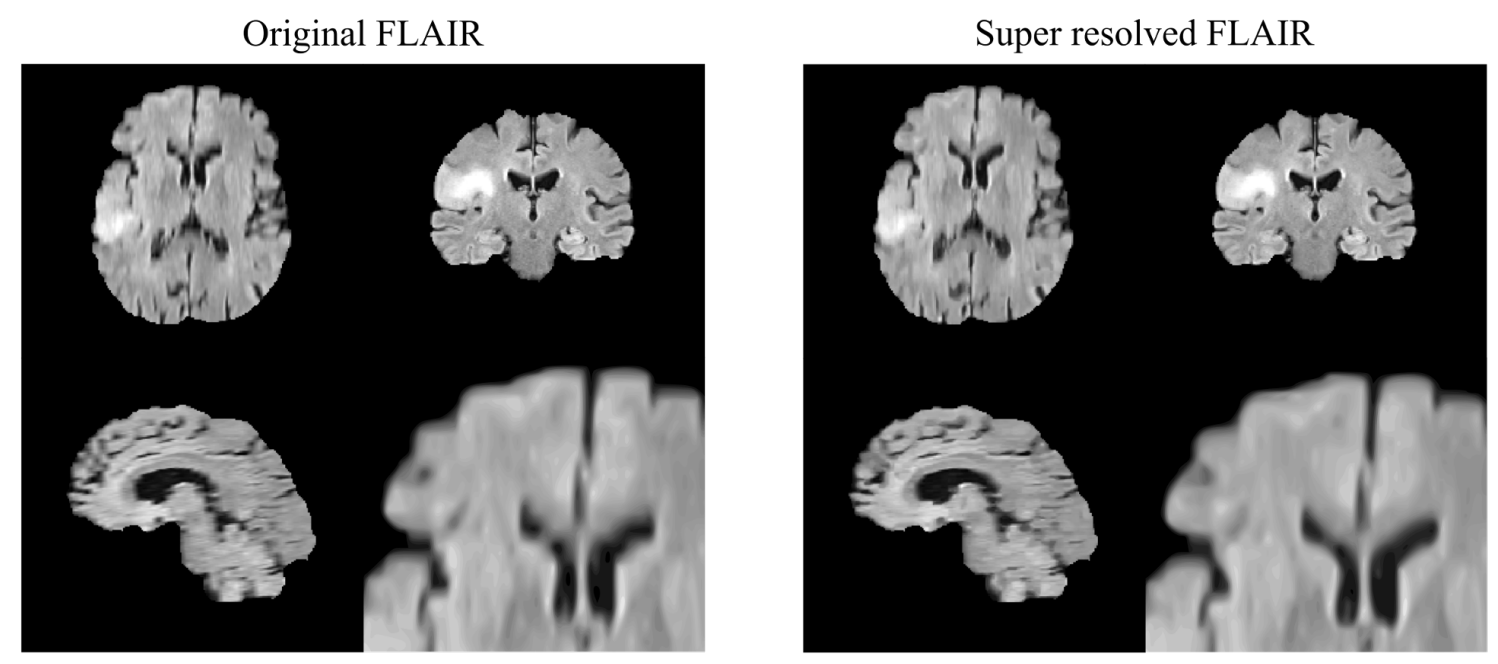

Figure 3.2: Example of super resolution using Non-local Upsampling method of a FLAIR sequence of the BRATS 2013 dataset. 


\subsubsection{Feature Extraction and Dimensionality Reduction}

Feature extraction comprises the process of obtaining new features from the MR images to improve discrimination between different labels. Although MRI intensities are the most common used features to differentiate between the brain tissues, it has been shown that including texture features in combination with MR intensities increases the performance of the segmentation algorithms (Kassner and Thornhill, 2010; Ahmed et al, 2011). In this sense, we have implemented the first order statistical texture features, also called histogram derived metrics or first order central moments.

For each patient we initially obtained an additional image, named $\mathrm{T}_{1 \text { Diff }}$, which consists on the absolute difference between the $T_{1 C E}$ and the $T_{1}$ images (Prastawa et al, 2003). This image highlights the contrast enhanced areas of the patient, such as the active tumor, helping in their discrimination. Next, for each MR image of the patient $\left(\mathrm{T}_{1}, \mathrm{~T}_{1 C E}, \mathrm{~T}_{2}\right.$, FLAIR and $\left.\mathrm{T}_{1 \text { Diff }}\right)$, we computed their first order texture features. Such features consist on the computation of local histograms in 3D patches centered at each voxel of the image, and calculate the mean, skewness and kurtosis of these histograms. We used local 3D patches of $5 \times 5 \times 5$ voxels.

Thus, a set $X$ of 20 images is obtained for each patient, consisting on the following images:

$$
X=\left(T_{1}, T_{1 C E}, T_{2}, F L A I R, T_{1 D i f f}, \mu T_{1}, \ldots, \gamma T_{1}, \ldots, \kappa T_{1 D i f f}\right)
$$

where $\mu, \gamma$, and $\kappa$ prefixes refers to the mean, skewness and kurtosis features of the corresponding image.

In order to reduce the complexity and number of parameters to estimate in the models, a dimensionality reduction process was performed. Dimensionality reduction seeks for an efficient representation of the original high dimensional data into a lower dimensional space, retaining or increasing the most relevant information. In our study, we used Principal Component Analysis (PCA) for dimensionality reduction. We run PCA on the $X$ set and selected the principal components, which together explained at least the $99 \%$ of the variance of the data, reducing in most cases from 20 dimensions to 5 dimensions. These images make up the final stack of imaging data used for the posterior unsupervised segmentations.

An slice example of the feature extraction and PCA dimensionality reduction process of a MR study is shown in Figure 3.3.

\subsubsection{Unsupervised voxel classification}

The BRATS 2013 dataset comprises 5 labels to be segmented, which in some cases a single label encloses different brain tissues (for example 0 or 1 label). This intraclass heterogeneity can severely affect the performance of unsupervised methods, since heterogeneity is naturally explained in unsupervised learning through the definition of different clusters. Hence, the same semantic class in a problem can be modeled through a set of different clusters.

In this sense, in order to increase the expression capacity of the unsupervised models to capture such heterogeneity, we assumed that each tissue can be initially represented by 2 clusters. That is, we will assume that there exist 7 tissues in the brain, which are labels 1, 2, 3 and 4 proposed in BRATS 2013 Challenge plus Grey Matter (GM), 


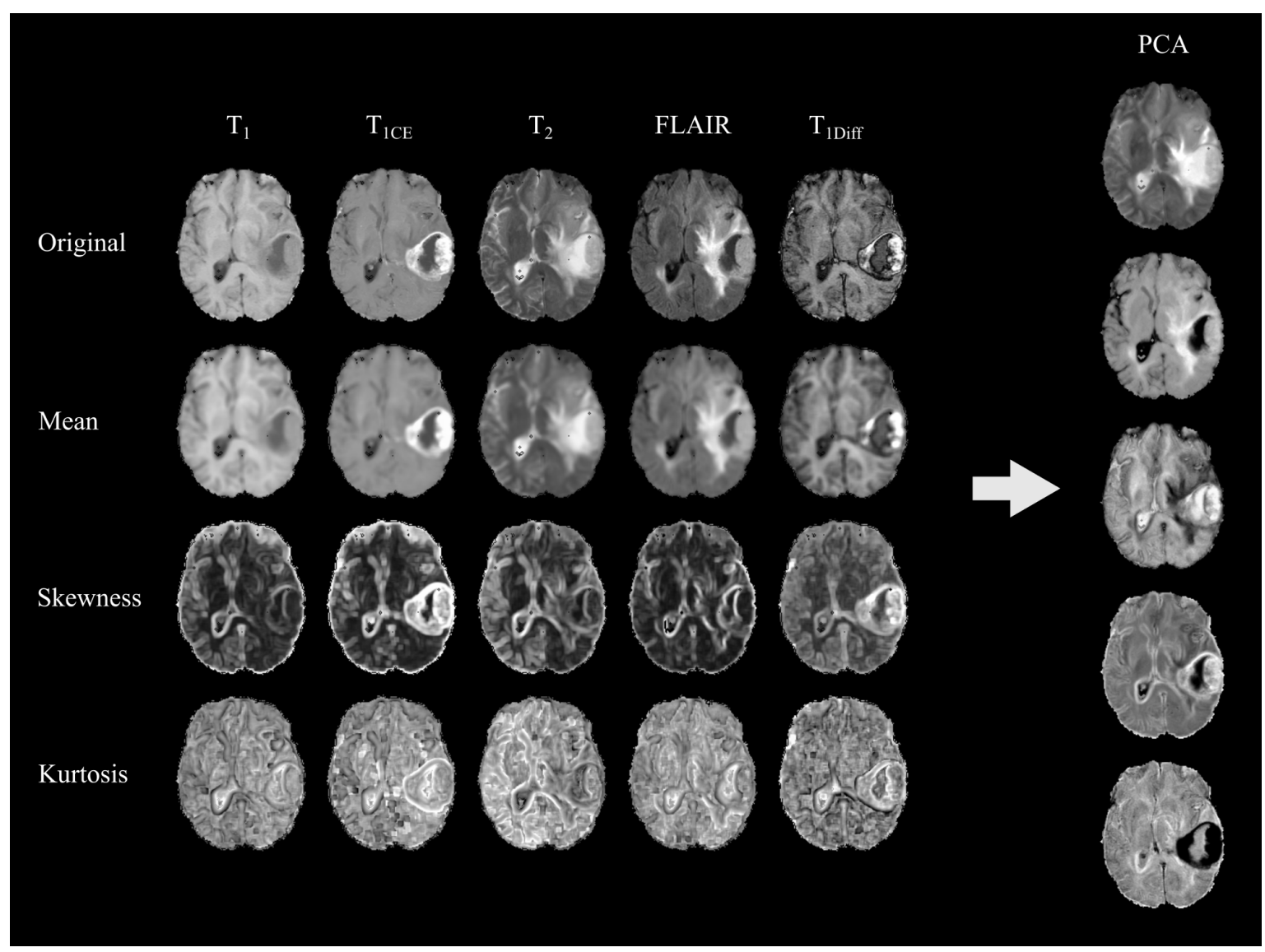

Figure 3.3: Example of feature extraction and dimensionality reduction of a MRI study of a patient of the BRATS 2013 dataset.

White Matter (WM) and Cerebro-Spinal Fluid (CSF); each one of them with initially 2 clusters to represent their heterogeneity. Note that, therefore, we will estimate an unsupervised clustering of 14 classes throughout the brain, but we do not require each tissue to use exactly 2 classes. Hence, a tissue showing a high degree of heterogeneity can be modeled using a set of 3 or 4 clusters, while an homogeneous tissue can be segmented by a single cluster. Such assumption provided us a balance between the number of parameters to estimate to the models and the degrees of freedom required to explain the heterogeneity of the tissues.

We evaluated the most popular unsupervised classification algorithms. We divided the algorithm comparison in two groups: structured and non-structured methods. Nonstructured algorithms classify data assuming an i.i.d. condition between the voxels of the images. Structured prediction covers the range of algorithms that involve the classification of data with a specific structure, such as an image. Under the non-structured paradigm, we evaluated three methods: K-means, Fuzzy K-means and GMM clustering. In the structured prediction case we evaluated the Gauss-HMRF as the archetype of unsupervised structured learning models.

Let $X=\left\{\mathbf{x}^{1}, \mathbf{x}^{2}, \ldots, \mathbf{x}^{N}\right\}$ the set of voxels to be classified, where $\mathbf{x}^{i} \in \mathbb{R}^{D}$ represents a feature vector of $D$ dimensions for the $i^{t h}$ voxel. Let $Y=\left\{y^{1}, y^{2}, \ldots, y^{N}\right\}$ the set of labels associated to each voxel, where $y^{i} \in\{1, \ldots, K\}$. 


\section{K-means}

K-means (Lloyd, 1982; Macqueen, 1967) is an unsupervised non-structured iterative partitional clustering based on a distance minimization criterion. Its aim is to divide the data space $X$ into $K$ clusters $\Pi=\left\{\Pi_{1}, \Pi_{2}, \ldots, \Pi_{K}\right\}$, so that each observation of $X$ belongs to the cluster with nearest centroid. The distance criterion minimized by $\mathrm{K}$-means is:

$$
\underset{\Pi}{\arg \min } \sum_{j=1}^{K} \sum_{\mathbf{x}^{i} \in \Pi_{j}}\left\|\mathbf{x}^{i}-\mu_{j}\right\|^{2}
$$

where $\mu_{j}$ represents the centroid of the $j^{\text {th }}$ cluster.

The K-means algorithm performs as follow:

Initialization: Make an initial guess about the cluster centroids $\mu_{j}$

Until there are no changes in centroids $\mu_{j}$ (or in clusters $\Pi$ )

Assign $y^{i}$ to the cluster with nearest centroid $\mu_{j}$ :

$$
y^{i}=\underset{j}{\arg \min }\left\|\mathbf{x}^{i}-\mu_{j}\right\|^{2}
$$

Update cluster centroids $\mu_{j}$

$$
\mu_{j}=\frac{1}{\left|\Pi_{j}\right|} \sum_{\mathbf{x}^{i} \in \Pi_{j}} \mathbf{x}^{i}
$$

From a statistical point of view, the iterative distance minimization criterion followed by K-means is equivalent to find the most likelihood parameters of a GMM (Duda et al, 2000), assuming shared identity covariance matrices and uniform prior probabilities for all classes. The iterative approach followed by K-means is also demonstrated a special limit of the EM algorithm (Dempster et al, 1977; Bishop, 1995), called Hard$E M$, where each observation is uniquely assigned to a class with posterior probability equal to 1 .

\section{Fuzzy K-means}

Likewise K-means, Fuzzy K-means (Dunn, 1973; Bezdek, 1981) is a non-structured iterative partitional clustering base on a distance minimization criterion. Under a probabilistic paradigm, it is also equivalent to a GMM assuming shared identity covariance matrices and uniform prior probabilities for all classes. However, Fuzzy K-means differs from K-means in which the assignment of an observation to a cluster is not hard but fuzzy. This means that each observation keeps a degree of membership to each cluster (equivalent to the posterior probability of a GMM) rather than a unique assignment of the observation to a class with posterior probability equal to 1 .

In the same manner as K-means, Fuzzy K-means aims to divide the data space $X$ into $K$ clusters $\Pi=\left\{\Pi_{1}, \Pi_{2}, \ldots, \Pi_{K}\right\}$, but it also keeps a vector $\mathbf{u}^{i}$ for each observation 
that determines the degree of membership of the $i^{\text {th }}$ observation to the different clusters. The distance minimization criterion followed by Fuzzy K-means is:

$$
\underset{\Pi}{\arg \min } \sum_{j=1}^{K} \sum_{i=1}^{N} u_{j}^{i}\left\|\mathbf{x}^{i}-\mu_{j}\right\|^{2}
$$

where $\mu_{j}$ represents the centroid of the $j^{\text {th }}$ cluster.

The membership variable $u_{j}^{i}$ is typically defined as:

$$
u_{j}^{i}=\frac{1}{\sum_{k=1}^{K}\left(\frac{\left\|\mathbf{x}^{i}-\mu_{j}\right\|^{2}}{\left\|\mathbf{x}^{i}-\mu_{k}\right\|^{2}}\right)^{\frac{2}{m-1}}}
$$

where $m$ controls the degree of fuzziness of the $j^{\text {th }}$ cluster, with $1 \leq m<\infty$ and typically set to 2 in absence of domain knowledge.

The Fuzzy K-means algorithm performs as follow:

Initialization: Make an initial guess about the cluster centroids $\mu_{j}$

Until there are no changes in $u_{j}^{i}$ greater than $\epsilon$

Estimate the membership coefficients $u_{j}^{i}$ for each observation and cluster:

$$
u_{j}^{i}=\frac{1}{\sum_{k=1}^{K}\left(\frac{\left\|\mathbf{x}^{i}-\mu_{j}\right\|^{2}}{\left\|\mathbf{x}^{i}-\mu_{k}\right\|^{2}}\right)^{\frac{2}{m-1}}}
$$

Update cluster centroids $\mu_{j}$ as the expected value of the observations given $u_{j}^{i}$ :

$$
\mu_{j}=\frac{\sum_{i=1}^{N} u_{j}^{i} \mathbf{x}^{i}}{\sum_{i=1}^{N} u_{j}^{i}}
$$

\section{Gaussian Mixture Model}

A GMM is probabilistic model that model a random variable as a convex combination of Gaussian pdfs. Thus, it provides a statistical framework to describe the heterogeneity of a dataset trough a weighted sum of single distributions, each one representing a subpopulation within a data. An in-depth explanation of FMMs and particularly GMMs is described in section 2.4. Please refer to this section for more details.

As a short remainder, the GMM is defined as:

$$
p(X ; \Theta)=\prod_{i=1}^{N} \sum_{j=1}^{K} \pi_{j} \mathcal{N}\left(\mathbf{x}^{i} ; \mu_{j}, \Sigma_{j}\right)
$$

where $\Theta=\left\{\mu_{1}, \ldots, \mu_{K}, \Sigma_{1}, \ldots, \Sigma_{K}, \pi_{1}, \ldots, \pi_{K}\right\}$ are the parameters of the model. The parameters $\left\{\pi_{1}, \ldots, \pi_{K}\right\}$ are typically called mixing coefficients and can be seen as the prior probability of each component of the mixture describing the data. 
Estimation on GMMs is performed via EM algorithm (Dempster et al, 1977) because direct MLE estimate does not yield closed-form solutions for the parameters of the model. For a complete derivation of the MLE estimate and the EM procedure in FMMs please refer to section 2.4. The algorithm for GMM clustering performs as follow:

Initialization: Choose an initial setting for $\Theta^{(0)}$.

Expectation step: Estimate $p\left(Z \mid X ; \Theta^{(t)}\right)$

$$
p\left(z_{j}^{i}=1 \mid \mathbf{x}^{i} ; \Theta^{(t)}\right)=\gamma_{j}^{i(t)}=\frac{\pi_{j}^{(t)} \mathcal{N}\left(\mathbf{x}^{i} ; \mu_{j}^{(t)}, \Sigma_{j}^{(t)}\right)}{\sum_{k} \pi_{k}^{(t)} \mathcal{N}\left(\mathbf{x}^{i} ; \mu_{k}^{(t)}, \Sigma_{k}^{(t)}\right)}
$$

Maximization step: Update the parameters of the model given $p\left(Z \mid X ; \Theta^{(t)}\right)$

$$
\hat{\Theta}_{M L E}^{(t+1)}=\underset{\Theta}{\arg \max } \mathbb{E}_{p\left(Z \mid X ; \Theta^{(t)}\right)} \log \mathcal{L}(\Theta ; X, Z)
$$

where sticking to GMMs:

$$
\begin{aligned}
\pi_{j}^{(t+1)} & =\frac{\sum_{i=1}^{N} \gamma_{j}^{i(t)}}{\sum_{i=1}^{N} \sum_{k=1}^{K} \gamma_{k}^{i(t)}} \\
\mu_{j}^{(t+1)} & =\frac{1}{\sum_{i=1}^{N} \gamma_{j}^{i(t)}} \sum_{i=1}^{N} \gamma_{j}^{i(t)} \mathbf{x}^{i} \\
\Sigma_{j}^{(t+1)} & =\frac{1}{\sum_{i=1}^{N} \gamma_{j}^{i(t)}} \sum_{i=1}^{N} \gamma_{j}^{i(t)}\left(\mathbf{x}^{i}-\mu_{j}^{(t+1)}\right)\left(\mathbf{x}^{i}-\mu_{j}^{(t+1)}\right)^{T}
\end{aligned}
$$

Convergence: Stop if $\mathcal{L}\left(\Theta^{(t+1)} ; X\right)-\mathcal{L}\left(\Theta^{(t)} ; X\right) \leq \epsilon$; otherwise $t=t+1$ and go to Expectation step.

As stated above, GMM clustering can be seen as the generalization of K-means and Fuzzy K-means algorithms, where the hard constraints related to the shared covariance matrices and the uniform prior probabilities are dropped.

\section{Gauss-Hidden Markov Random Field}

MRFs are probabilistic undirected graphical models that define a family of joint probability distributions by means of an undirected graph (Hammersley and Clifford, 1971). These graphs are used to introduce conditional dependencies between random variables of the model, which in the case of the image segmentation task, allows capturing 
the self-similarity and local redundancy present in the images. These dependencies are explicitly denoted via an undirected and cyclic graph, whose vertices represent the pixels/voxels of the images and whose edges represent the dependencies between them. In this sense, a generative model with a given set of parameters $\Theta$ incorporating a HMRF can be defined as:

$$
\begin{aligned}
p(X ; \Theta) & =\sum_{Y} p(X, Y ; \Theta) \\
& =\sum_{Y} p(Y) p(X \mid Y ; \Theta)
\end{aligned}
$$

MRFs are usually used to model the prior density $p(Y)$ of a probabilistic generative model. According to the Hammersley-Clifford theorem, assuming that the prior density is strictly positive, a MRF can be defined in terms of local energy functions and therefore it can be expressed as a Gibbs measure in the form:

$$
p(Y)=\frac{1}{Z} \exp (-U(Y))
$$

where $U(Y)$ is an energy function that defines the conditional dependencies between the random variables via the graphical model, and $Z$ is called the partition function and acts as a normalizer to ensure the density to sum 1:

$$
Z=\sum_{Y^{\prime}} \exp \left(-U\left(Y^{\prime}\right)\right)
$$

Hammersey-Clifford theorem also states that $p(Y)$ can be factorized over the cliques of the undirected graphical model. A clique is defined as a subset of vertices in the graph such that there exist an edge between all pairs of vertices in the subset. Let $\mathcal{Q}$ the set of all cliques of the graph, the energy function $U(Y)$ is defined as:

$$
U(Y)=\sum_{q \in \mathcal{Q}} \Psi(Y(q))
$$

Nowadays, if complexity is considered, the inference algorithms for MRFs are only able to optimize undirected graphs with cliques of order 2 (pairwise cliques), i.e. $q \rightarrow$ $\left(y^{n}, y^{m}\right) \quad \forall q \in \mathcal{Q}$. Hence, the most widely used graphical model is the Ising model. The Ising model defines a graph lattice with as many vertices as pixels/voxels exist in the image, where conditional dependencies of each variable are expressed in terms of its orthogonal adjacent neighborhood. The clique factorization for the Ising model is then performed in the form:

$$
\begin{aligned}
U(Y) & =\sum_{\left(y^{n}, y^{m}\right) \in Q} \Psi\left(y^{n}, y^{m}\right) \\
& =\sum_{\left(y^{n}, y^{m}\right) \in Q} \beta^{(n, m)} \delta\left(y^{n}, y^{m}\right)
\end{aligned}
$$


where $\beta^{(n, m)}$ is a weight defined for the corresponding clique, and $\delta$ is a function that measures the dissimilarity between the clique. Typically, in absence of domain knowledge, $\beta^{(n, m)}$ is usually set to 1 and $\delta$ is defined as:

$$
\delta\left(y^{n}, y^{m}\right)= \begin{cases}0 & \text { if } y^{n}=y^{m} \\ 1, & \text { otherwise }\end{cases}
$$

Typically, the class conditional density $p(X \mid Y ; \Theta)$ is also expressed in terms of Gibbs measures to take advantage from MRF solvers, so it is usually rewritten as:

$$
p(X \mid Y ; \Theta)=\frac{1}{Z} \exp (-U(X \mid Y ; \Theta))
$$

where $U(X \mid Y ; \Theta)$ can be assumed i.i.d. and is proportional to the class conditional $p(X \mid Y ; \Theta)$ density, and $Z$ is again a partition function to ensure the distribution to sum 1. Under a Gaussian model, $U(X \mid Y ; \Theta)$ can be defined as:

$$
U(X \mid Y ; \Theta)=\sum_{i=0}^{N} U\left(\mathbf{x}^{i} \mid y^{i}=j ; \Theta\right)=\sum_{i=0}^{N}-\log \mathcal{N}\left(\mathbf{x}^{i} ; \mu_{j}, \Sigma_{j}\right)
$$

As a results, the complete structured model is defined as:

$$
p(X ; \Theta)=\sum_{Y} \frac{1}{Z} \exp (-U(X \mid Y ; \Theta)-U(Y))
$$

Exact inference on this model is intractable due to the sum over all possible set of labels in $Z$, which becomes a \#P-complete problem. However, approximate efficient algorithms to compute the best labeling $\hat{Y}$ are proposed in the literature. Iterated Conditional Modes (Birchfield and Tomasi, 1998), Monte Carlo Sampling (Geyer and Thompson, 1992), Loopy Belief Propagation (Yedidia et al, 2003), Mean Field Approximation (Parisi, 1998) or Graph cuts (Boykov et al, 2001) are some of the available algorithms to solve pairwise MRFs-based models. In our study we used the FastPD algorithm proposed by Komodakis and Tziritas (2007); Komodakis et al (2008), which has been demonstrated to be superior to most of the aforementioned algorithms. This algorithm is based on a combination of Graph cuts with primal-dual strategies. Therefore the best labeling is computed as:

$$
\hat{Y}=\arg \max -U(X \mid Y ; \Theta)-U(Y)
$$

In this sense, we can think of $\hat{Y}$ as a binary one-hot encoding variable holding the posterior probability of each label for each pixel/voxel, with $\hat{y}_{j}^{i}=1$ for the winner class and $\hat{y}_{k}^{i}=0, \quad k \neq j$ for the remaining classes.

Therefore, likewise GMM, Gauss-HMRF also finds the MLE parameters of a mixture of Gaussian distributions that better fits the data, but imposing the structured MRF prior. A Hard-EM version (given that exact inference is not possible) of the EM algorithm is employed to estimate the parameters of the model in the form: 
Initialization: Choose an initial setting for $\Theta^{(0)}$.

Expectation step: Estimate $\hat{Y}$ by using FastPD (or similar) algorithm

$$
\begin{aligned}
U(Y) & =\sum_{\left(y^{n}, y^{m}\right) \in Q} \beta^{(n, m)} \delta\left(y^{n}, y^{m}\right) \\
U\left(X \mid Y ; \Theta^{(t)}\right) & =\sum_{i=0}^{N}-\log \mathcal{N}\left(\mathbf{x}^{i} ; \mu_{j}^{(t)}, \Sigma_{j}^{(t)}\right) \\
\hat{Y} & =\arg \max -U\left(X \mid Y ; \Theta^{(t)}\right)-U(Y)
\end{aligned}
$$

Maximization step: Update the parameters of the model given $\hat{Y}$

$$
\hat{\Theta}_{M L E}^{(t+1)}=\underset{\Theta}{\arg \max } \mathbb{E}_{\hat{Y}} \log \mathcal{L}(\Theta ; X, Z)
$$

where sticking to GMMs:

$$
\begin{aligned}
\pi_{j}^{(t+1)} & =\frac{\sum_{i=1}^{N} \hat{y}_{j}^{i}}{\sum_{i=1}^{N} \sum_{k=1}^{K} \hat{y}_{k}^{i}} \\
\mu_{j}^{(t+1)} & =\frac{1}{\sum_{i=1}^{N} \hat{y}_{j}^{i}} \sum_{i=1}^{N} \hat{y}_{j}^{i} \mathbf{x}^{i} \\
\Sigma_{j}^{(t+1)} & =\frac{1}{\sum_{i=1}^{N} \hat{y}_{j}^{i}} \sum_{i=1}^{N} \hat{y}_{j}^{i}\left(\mathbf{x}^{i}-\mu_{j}^{(t+1)}\right)\left(\mathbf{x}^{i}-\mu_{j}^{(t+1)}\right)^{T}
\end{aligned}
$$

Convergence: Stop if $\mathcal{L}\left(\Theta^{(t+1)} ; X\right)-\mathcal{L}\left(\Theta^{(t)} ; X\right) \leq \epsilon$; otherwise $t=t+1$ and go to Expectation step.

\section{Algorithms initialization}

A well-known requirement of unsupervised learning is the good initial seeding. Although the global minima is not usually reached even if a good initialization is provided, a bad initialization can lead the model to a very sub-optimal local minimum, thereby providing a poor segmentation. Several strategies such as multiple replications or intelligent initial seeding are proposed to palliate this effect. In our study, we implemented the K-means ++ algorithm (Arthur and Vassilvitskii, 2007), which provides an initialization that attempts to avoid local sub-optimal minimums.

We propose the following procedure to ensure a competitive unsupervised segmentation: First, generate 100 different initializations using $K$-means ++ algorithm. Next, automatically select the 10 most promising initializations by minimizing the average 
intra-cluster sums of point-to-centroid distances of the initializations. Finally, run each unsupervised segmentation algorithm with the 10 most promising initializations and choose the best solution considering the following criteria:

K-means: choose the solution with lowest intra-cluster sums of point-to-centroid distances.

Fuzzy K-means: choose the solution with lowest intra-cluster sums of point-to-centroid distances.

GMM: choose the solution with highest log-Likelihood value.

Gauss-HMRF: choose the solution with highest log-Likelihood value.

\subsubsection{Automated pathological label identification}

Unsupervised segmentation produces a partitioning of the data space into several classes, each one without semantic sense. In other words, in the brain tumor unsupervised approach, labels do not directly identify a specific tissue but only distinguish between MRI data different enough from each other to be considered equal. Moreover, labelings among different patient segmentations may not always represent the same tissue, complicating its biological interpretation. Hence, an automated pathological label identification is mandatory to provide a powerful and competitive unsupervised segmentation method. We propose the following method to automatically isolate pathological labels:

1. Identify and remove WM, GM and CSF labels.

2. Remove outlier and partial volume labels.

3. Merge labels by statistical distribution similarities.

\section{Identify and remove WM, GM and CSF labels}

Under the International Consortium of Brain Mapping (ICBM) project, an unbiased standard MR brain atlas was provided by the McConnell Brain Imaging Centre in 2009 (Fonov et al, 2011, 2009). The ICBM atlas include a $\mathrm{T}_{1}, \mathrm{~T}_{2}$ and Proton density MR images, with the associated WM, GM and CSF tissue probability maps. Such tissue probability maps indicate the probability for each voxel $v$ of the brain to belong to a normal tissue $T=\{W M, G M, C S F\}$, with:

$$
\sum_{t \in T} p(t \mid v)=1
$$

In our study we used these tissue probability maps to detect which labels of a segmentation explain the WM, GM and CSF tissues. However, taking into account that the ICBM template represents a healthy brain, it is necessary to corrected these maps by setting to zero (or a smaller $\epsilon$ value) the probability of any voxel $v$ in the area of the lesion. Therefore, we first performed a non-linear registration of the ICBM $\mathrm{T}_{1}$ template to the $\mathrm{T}_{1}$ sequence of the patient and applied the warp transformation to 
the tissue probability maps. Following the study conducted by Klein et al (2009), we used the SyN algorithm (Avants et al, 2008) implemented in the ANTS software with cross-correlation similarity metric. Once we obtained the patient aligned healthy-tissue probability maps, a roughly approximate mask of the lesion of each patient was computed to correct the probability maps. The typical delineation of the lesion performed by expert radiologists is usually based on the hyper-intensity areas in the $\mathrm{T}_{2}$ and $\mathrm{T}_{1 C E}$ sequences (Bauer et al, 2013). Following a similar criteria, we computed an approximate mask of the lesion by selecting the voxels whose value in the FLAIR and $\mathrm{T}_{1 C E}$ images were higher than the median plus the standard deviation of the corresponding image. Next, we automatically filled the holes of the computed masks and removed the voxels that fell in the perimeter of the brain (typically showing hyper-intensities due to cranial traces). Finally, we corrected the healthy-tissue probability maps of each patient by setting an $\epsilon$ probability in the area determined by their corresponding lesion masks. Figure 3.4 shows an illustration of the algorithm to compute the corrected tissue probability maps for a patient.

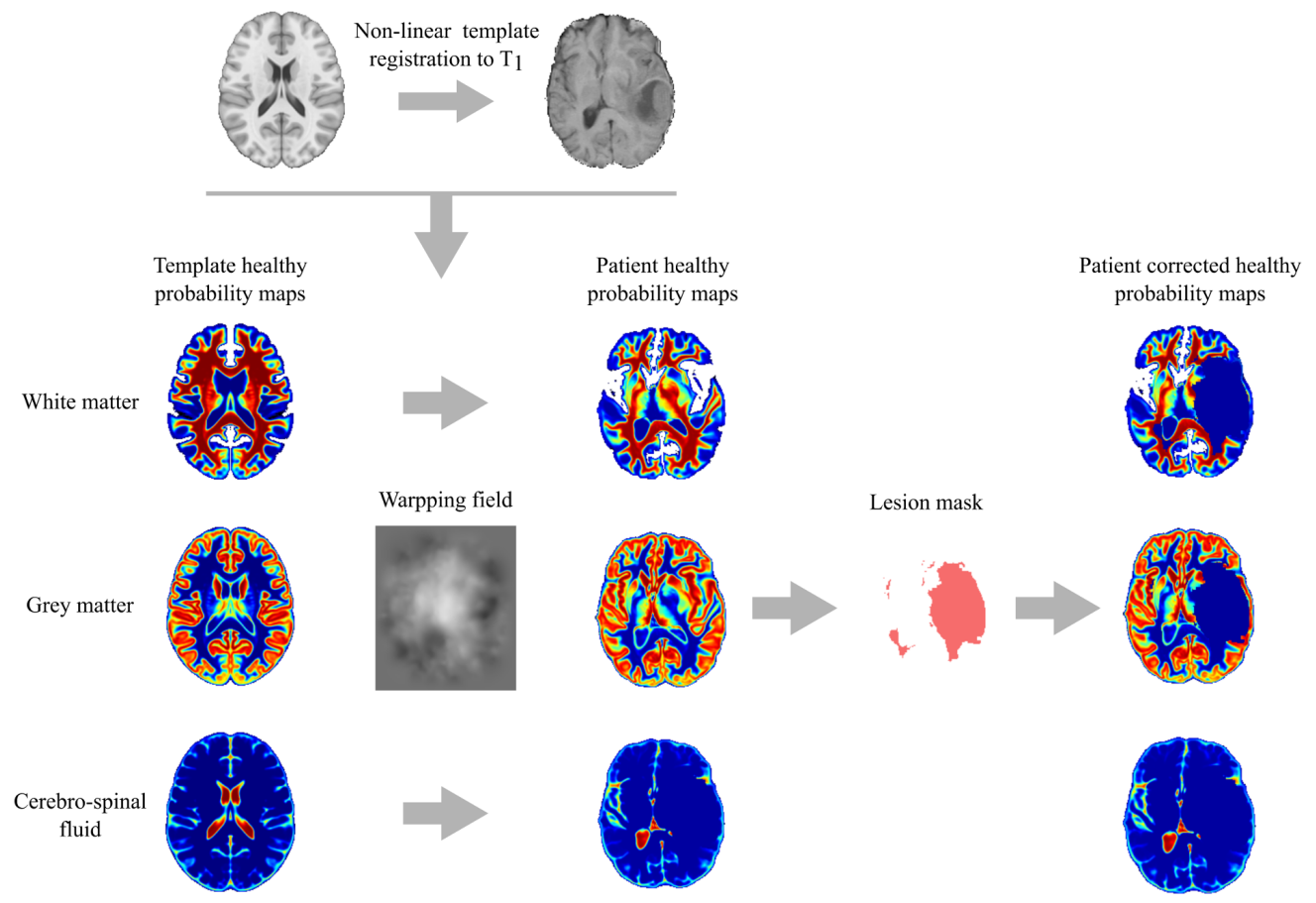

Figure 3.4: Illustration of the algorithm to compute the patient healthy-tissue probability maps and lesion area correction.

Based on these patient healthy-tissue probability maps, we identified which classes of an unsupervised segmentation mostly represent a normal tissue. Let $S$ a segmentation obtained through any unsupervised method, and let $v_{l}=\{v: S(v)=l\}$ the set of voxels $v$ of $S$ classified with label $l$. Let $t$ a normal tissue where $t \in T$. To perform the pathological tissue identification we computed the following probability mass: 


$$
p(l \mid t, S)=\frac{\sum_{u \in v_{l}} p(t \mid u)}{\sum_{v} p(t \mid v)}
$$

Simplifying, the $p(c \mid t, S)$ determines how much of the normal tissue $t$ is explained by the label $l$ in the segmentation $S$.

Therefore, for a given tissue $t$, and based on these probabilities, we constructed two vectors: one with the $p(c \mid t, S)$ values sorted in descending order, denoted as $\mathcal{P}_{t}$, and the other with the corresponding label codes sorted in the same manner, denoted as $\mathcal{K}_{t}$.

$$
\begin{aligned}
& \mathcal{K}_{t}=\left\{l: p(l \mid t, S) \geq p\left(l^{\prime} \mid t, S\right)\right\} \\
& \mathcal{P}_{t}=\left\{p(l \mid t, S): p(l \mid t, S) \geq p\left(l^{\prime} \mid t, S\right)\right\}
\end{aligned}
$$

Then, we computed the cumulative sum of $\mathcal{P}_{t}$, denoted as $\mathcal{S}_{t}$, and finally choose the set of labels of $\mathcal{K}_{t}$ whose $\mathcal{S}_{t}$ value exceed a threshold $\tau$.

$$
\mathcal{Z}_{t}=\left\{\mathcal{K}_{t}(j): \mathcal{S}_{t}(j)>\tau, \quad 1 \leq j<K\right\}
$$

The $\mathcal{Z}_{t}$ set contains the classes of $S$ that have a very low probability of explain the normal tissue $t$. Hence, we repeated the same procedure for each normal tissue $t$ and computed the intersection between the $\mathcal{Z}_{t}$ sets to finally isolate the labels that do not explain any normal tissue, i.e. the pathological classes:

$$
\mathcal{Z}=\mathcal{Z}_{W M} \cap \mathcal{Z}_{G M} \cap \mathcal{Z}_{C S F}
$$

Given that our aim is to evaluate the performance of each unsupervised segmentation algorithm, all of them in the same conditions, we do not carried out any particular optimization of the $\tau$ threshold for each algorithm. Instead, we fixed a general threshold for all the methods, set to $\tau=0.8$, as a reasonably, high confidence and compatible value to perform the pathological labels identification. Note that $\tau=1$ is not possible since this implies that all the classes of the segmentation will be required to explain only a single normal tissue.

\section{Remove outlier and partial volume labels}

The above process of identifying and removing the healthy-tissue labels may leave some spurious labels that must be deleted. We found that these labels frequently appear in the perimeter of the brain or in a very low proportion compared to the other labels of the segmentation. The labels located at the perimeter of the brain typically correspond to remaining traces of cranium, intensity artifacts between brain and background or partial volume effects that super resolution cannot resolve. The low proportion labels often match to outlier voxels in terms of abnormal intensity values, usually produced by artifacts in the MR acquisition.

In order to remove the perimeter unwanted labels, we deleted all the connected components of all the remaining labels of the segmentation, which overlapped more than the $50 \%$ of its area with a binary dilated mask of the perimeter of the brain. To 
remove the smaller low proportion labels we removed those ones with a prevalence less than the $1 \%$ the remaining segmentation after the above processes.

\section{Merge labels by statistical distribution similarities}

The heterogeneity of the pathological labels led us to assume that each tissue was initially modeled by two clusters. However, this was a general but not strict assumption, i.e. we did not enforce to use exactly two cluster per tissue. Instead, we estimated a general clustering of 14 components for each case. Hence, a tissue may have been represented by two or more labels in the segmentation or, conversely, by a single label depending on its homogeneity. Therefore, it was mandatory to design a mechanism to find which labels were explaining the same semantic concept, i.e. the same pathological tissue.

Based on the work proposed by Sáez et al (2017), we analyzed the MRI intensity distributions of the remaining labels to find potential clusters representing the similar information. To do so, we estimated the pdf of each label through a kernel smoothing density estimation, and used the Jensen-Shannon divergence to measure the pairwise distances among them. Therefore, we constructed a pairwise matrix of statistical distribution distances and used Hierarchical Agglomerative Clustering (HAC) with average link (Unweighted Pair Group Method with Arithmetic mean (UPGMA)) to merge the similar labels.

Due to the BRATS 2013 labeling considers 4 pathological labels to be segmented, we enforced the clustering to return a maximum of 4 classes. Note that the method is able to return less than 4 classes if the HAC finds enough similarities between them, however, in any other case the method is enforced to return a maximum of 4 labels. Figure 3.5 shows and example of the pathological labels isolation procedure.

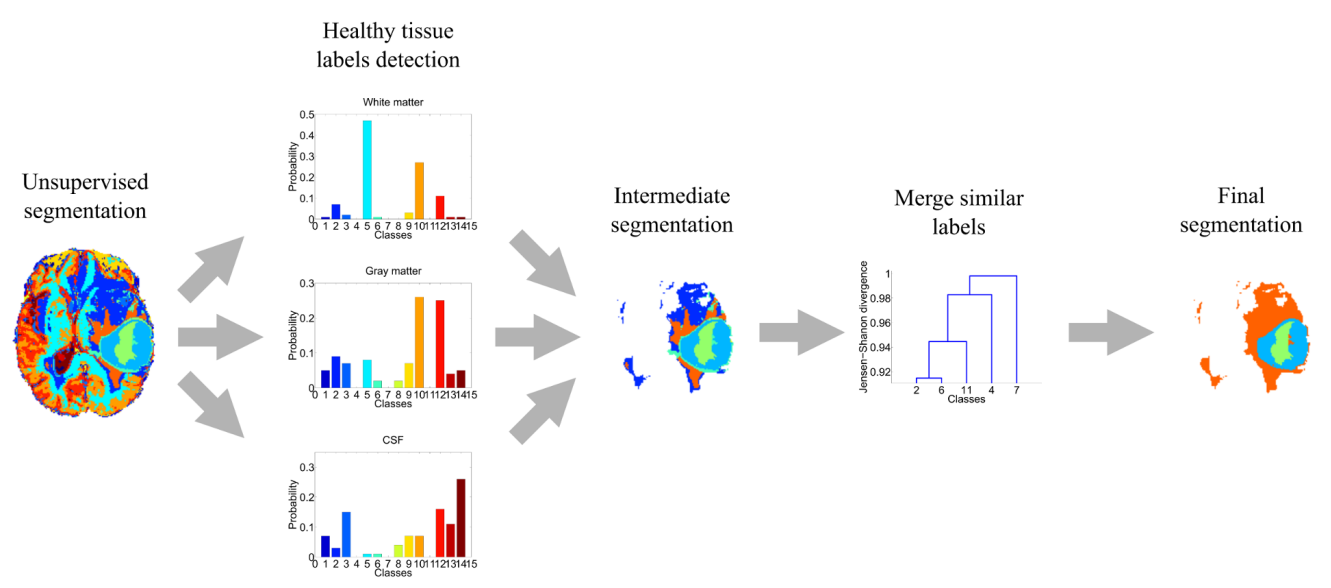

Figure 3.5: Automated pathological label identification process.

\subsubsection{Evaluation}

We evaluated our unsupervised brain tumor segmentation framework with the BRATS 2013 Leaderboard and Test datasets. Segmentations provided by the different unsu- 
pervised methods in combination with the proposed preprocessing and postprocessing pipelines were sent to the BRATS evaluation web page. The figures of merit provided to assess the quality of the segmentations were:

- Dice: $\frac{2(T P+T N)}{P+N+\hat{P}+\hat{N}}$

- PPV: $\frac{T P}{T P+F P}$

- Sensitivity: $\frac{T P}{T P+F N}$

- Kappa: $\frac{P_{A}-P_{E}}{1-P_{E}}$

where $T P$ refers to the true positives in the segmentation, $T N$ to the true negatives, $F P$ to the false positives, $F N$ to the false negatives, $P$ to the real positives of the ground truth, $N$ to the real negatives of the ground truth, $\hat{P}$ to the estimated positives of the proposed segmentation, $\hat{N}$ to the estimated negatives of the proposed segmentation, $P_{A}$ to the accuracy of the segmentation and $P_{E}$ to a term that measures the probability of success by chance, defined as: $P_{E}=\left(\frac{P}{P+N} \cdot \frac{\hat{P}}{\hat{P}+\hat{N}}\right)+\left(\frac{N}{P+N} \cdot \frac{\hat{N}}{\hat{P}+\hat{N}}\right)$.

Furthermore, three different sub-compartments of the lesion were evaluated to properly assess the quality of the segmentation methods. Table 3.1 describes the labels involved in each sub-compartment considered in the evaluation.

Table 3.1: Labels composing each sub-compartment evaluated in the BRATS 2013 challenge.

\begin{tabular}{lccccc} 
& Label 0 & Label 1 & Label 2 & Label 3 & Label 4 \\
Whole Tumor (WT) & & $\times$ & $\times$ & $\times$ & $\times$ \\
Tumor Core (TC) & & $\times$ & & $\times$ & $\times$ \\
Enhancing Tumor (ET) & & & & & $\times$ \\
\hline
\end{tabular}

\subsection{Results}

Tables 3.2 and 3.3 show the results obtained in the Test and Leaderboard datasets respectively, grouped by the unsupervised algorithms tested in this study.

As expected, Gauss-HMRF and GMM demonstrate their superiority with respect the other algorithms. Almost all the metrics reveal that both algorithms obtain the best results in all the sub-compartments segmentations. Only the enhancing tumor sub-compartment in the Leaderboard set yielded worse results for the GaussHMRF compared to the results obtained in the other sub-compartments and datasets. Such effect typically occurs because of the smoothing prior of the Gauss-HMRF, which is later discussed in the Discussion section.

Tables 3.4 and 3.5 show the published ranking of the BRATS competition grouped by the learning paradigm adopted by each method and the metrics and sub-compartments evaluated in the Challenge. As shown in Table 3.4, we achieved the $1^{\text {st }}$ position in the 
Table 3.2: Summary of average results obtained by the different unsupervised algorithms in combination with the proposed preprocess and postprocess over the BRATS 2013 Test set.

\begin{tabular}{lcccccccccc}
\hline \multirow{2}{*}{ Classifier } & \multicolumn{3}{c}{ Dice } & \multicolumn{4}{c}{ PPV } & \multicolumn{4}{c}{ Sensitiviy } & Kappa \\
& WT & TC & ET & WT & TC & ET & WT & TC & ET & \\
\cline { 2 - 8 } & 0.69 & 0.49 & 0.57 & 0.66 & 0.48 & 0.68 & 0.76 & 0.57 & 0.51 & 0.98 \\
Kuzzy K-means & 0.70 & 0.46 & 0.39 & 0.73 & 0.47 & 0.51 & 0.71 & 0.54 & 0.35 & 0.98 \\
GMM & 0.69 & 0.60 & 0.55 & 0.63 & 0.60 & 0.64 & 0.78 & 0.68 & 0.55 & 0.98 \\
Gauss-HMRF & 0.72 & 0.62 & 0.59 & 0.68 & 0.58 & 0.67 & 0.81 & 0.75 & 0.60 & 0.98 \\
\hline
\end{tabular}

Table 3.3: Summary of average results obtained by the different unsupervised algorithms in combination with the proposed preprocess and postprocess over the BRATS 2013 Leaderboard set.

\begin{tabular}{lcccccccccc}
\hline \multirow{2}{*}{ Classifier } & \multicolumn{3}{c}{ Dice } & \multicolumn{4}{c}{ PPV } & \multicolumn{5}{c}{ Sensitiviy } & Kappa \\
& WT & TC & ET & WT & TC & ET & WT & TC & ET & \\
\cline { 2 - 9 } K-means & 0.76 & 0.49 & 0.53 & 0.75 & 0.44 & 0.66 & 0.82 & 0.56 & 0.48 & 0.99 \\
Fuzzy K-means & 0.77 & 0.46 & 0.25 & 0.81 & 0.46 & 0.27 & 0.77 & 0.51 & 0.27 & 0.99 \\
GMM & 0.74 & 0.59 & 0.60 & 0.71 & 0.55 & 0.60 & 0.81 & 0.71 & 0.66 & 0.99 \\
Gauss-HMRF & 0.77 & 0.63 & 0.32 & 0.72 & 0.61 & 0.33 & 0.84 & 0.71 & 0.50 & 0.99 \\
\hline
\end{tabular}

ranking of the unsupervised methods of the Test set, and the $7^{\text {th }}$ position in the general ranking, mostly against supervised approaches. Table 3.5 shows the Leaderboard ranking and the results achieved by our method. The proposed approach in combination with the GMM algorithm reaches the $2^{\text {nd }}$ position of the Leaderboard ranking, improving the results obtained by many supervised methods, mainly in the enhancing tumor sub-compartment.

Table 3.6 shows the average time in minutes required to obtain a segmentation for a single patient, including the preprocessing and postprocessing stages. Segmentations were computed in an Intel Xeon E5-2620 with 64GB of RAM using multi-threading. The preprocessing stage includes the denoising, bias field correction, skull-stripping and super resolution steps. The unsupervised classification time involves the parallel computation of the 10 different segmentations starting from the K-means++ initialization, and the posterior selection of the best solution. As expected, the more complex and sophisticated the algorithm is, the longer it takes to reach the solution. The postprocessing stage consist in the automated pathological label identification method, the outlier label removal and the merging process of similar statistical distribution labels. Such process includes the non-linear registration of the ICBM template to the patient $\mathrm{T}_{1}$ image, which practically covers the entire time of the postprocessing stage. It is worth noting that the non-linear ICBM registration is performed only once for all the unsupervised segmentation algorithms. 
Chapter 3. Comparative study of unsupervised learning algorithms for glioblastoma segmentation

Table 3.4: Ranking of the BRATS 2013 Test set and the position occupied by our proposed unsupervised segmentation framework with the Gauss-HMRF algorithm.

\begin{tabular}{|c|c|c|c|c|c|c|c|c|c|c|c|}
\hline & \multirow{2}{*}{ User } & \multicolumn{3}{|c|}{ Dice } & \multicolumn{3}{|c|}{$\mathrm{PPV}$} & \multicolumn{3}{|c|}{ Sensitiviy } & \multirow{2}{*}{ Kappa } \\
\hline & & WT & $\mathrm{TC}$ & ET & WT & $\mathrm{TC}$ & ET & WT & $\mathrm{TC}$ & ET & \\
\hline \multirow{6}{*}{$\begin{array}{c}\text { Supervised } \\
\text { methods }\end{array}$} & Nick Tustison & 0.87 & 0.78 & 0.74 & 0.85 & 0.74 & 0.69 & 0.89 & 0.88 & 0.83 & 0.99 \\
\hline & Raphael Meier & 0.82 & 0.73 & 0.69 & 0.76 & 0.78 & 0.71 & 0.92 & 0.72 & 0.73 & 0.99 \\
\hline & Syed Reza & 0.83 & 0.72 & 0.72 & 0.82 & 0.81 & 0.70 & 0.86 & 0.69 & 0.76 & 0.99 \\
\hline & Liang Zhao & 0.84 & 0.70 & 0.65 & 0.80 & 0.67 & 0.65 & 0.89 & 0.79 & 0.70 & 0.99 \\
\hline & Nicolas Cordier & 0.84 & 0.68 & 0.65 & 0.88 & 0.63 & 0.68 & 0.81 & 0.82 & 0.66 & 0.99 \\
\hline & Joana Festa & 0.72 & 0.66 & 0.67 & 0.77 & 0.77 & 0.70 & 0.72 & 0.60 & 0.70 & 0.98 \\
\hline \multirow{2}{*}{$\begin{array}{c}\text { Unsupervised } \\
\text { methods }\end{array}$} & This work & 0.72 & 0.62 & 0.59 & 0.68 & 0.58 & 0.67 & 0.81 & 0.75 & 0.60 & 0.98 \\
\hline & Senan Doyle & 0.71 & 0.46 & 0.52 & 0.66 & 0.38 & 0.58 & 0.87 & 0.70 & 0.55 & 10.98 \\
\hline
\end{tabular}

Table 3.5: Ranking of the BRATS 2013 Leaderboard set and the position occupied by our proposed unsupervised segmentation framework with the GMM algorithm.

\begin{tabular}{|c|c|c|c|c|c|c|c|c|c|c|c|}
\hline & \multirow{2}{*}{ User } & \multicolumn{3}{|c|}{ Dice } & \multicolumn{3}{|c|}{ PPV } & \multicolumn{3}{|c|}{ Sensitiviy } & \multirow{2}{*}{ Kappa } \\
\hline & & $\mathrm{WT}$ & $\mathrm{TC}$ & ET & $\mathrm{WT}$ & $\mathrm{TC}$ & $\mathrm{ET}$ & WT & $\mathrm{TC}$ & $\mathrm{ET}$ & \\
\hline $\begin{array}{c}\text { Supervised } \\
\text { method }\end{array}$ & Nick Tustison & 0.79 & 0.65 & 0.53 & 0.83 & 0.70 & 0.51 & 0.81 & 0.73 & 0.66 & 0.99 \\
\hline $\begin{array}{c}\text { Unsupervised } \\
\text { method }\end{array}$ & This work & 0.74 & 0.59 & 0.60 & 0.71 & 0.55 & 0.60 & 0.81 & 0.71 & 0.66 & 0.99 \\
\hline \multirow{4}{*}{$\begin{array}{l}\text { Supervised } \\
\text { methods }\end{array}$} & Liang Zhao & 0.79 & 0.59 & 0.47 & 0.77 & 0.55 & 0.50 & 0.85 & 0.77 & 0.53 & 0.99 \\
\hline & Raphael Meier & 0.72 & 0.60 & 0.53 & 0.65 & 0.62 & 0.48 & 0.88 & 0.69 & 0.64 & 0.99 \\
\hline & Syed Reza & 0.73 & 0.56 & 0.51 & 0.68 & 0.64 & 0.48 & 0.79 & 0.57 & 0.63 & 0.99 \\
\hline & Nicolas Cordier & 0.75 & 0.61 & 0.46 & 0.79 & 0.61 & 0.43 & 0.78 & 0.72 & 0.52 & 1.00 \\
\hline
\end{tabular}

Finally, examples of segmentations achieved by the different unsupervised segmentation algorithms evaluated are shown in Figure 3.6.

\subsection{Discussion}

In this study we have conducted an evaluation of the performance of several unsupervised learning algorithms for the glioblastoma segmentation task. In addition to the comparative, we have proposed a complete pipeline for automated brain tumor segmentation, including a postprocessing stage to automatically identify labels corresponding to pathological tissues in an unsupervised segmentation.

The proposed method is confirmed as a viable alternative for glioblastoma segmentation, as it has demonstrated to achieve competitive results in a public real reference dataset for brain tumor segmentation. The method improved the results obtained by the other unsupervised segmentation approaches evaluated in the BRATS 2013 Chal- 
Table 3.6: Average computational times in minutes for the whole segmentation pipeline for a single patient.

\begin{tabular}{|c|c|c|c|c|}
\hline Algorithm & Preprocess & Unsupervised classification & Postprocess & Total \\
\hline K-means & \multirow{4}{*}{$13 \pm 3$} & $9 \pm 5$ & \multirow{4}{*}{$88 \pm 19$} & $110 \pm 27$ \\
\hline Fuzzy K-means & & $29 \pm 3$ & & $130 \pm 25$ \\
\hline GMM & & $41 \pm 7$ & & $142 \pm 29$ \\
\hline Gauss-HMRF & & $39 \pm 10$ & & $140 \pm 32$ \\
\hline
\end{tabular}

lenge, and obtained competitive results with respect to supervised methods. Moreover, this study confirmed the capability of unsupervised learning to detect consistent patterns in medical imaging data related to MRI properties of the tissue, which will serve as basis for the next work on this thesis.

The proposed unsupervised segmentation pipeline comprises four stages: MRI preprocessing, feature extraction and dimensionality reduction, unsupervised voxel classification and automatic pathological label identification. Concerning the preprocessing stage, consolidated state of the art techniques that provide efficient solutions to enhance the information of the MR images were employed. However, some preprocessing techniques are primarily oriented to non-pathological brains. This is the case of bias field correction. In our experiments, we found that the estimation of the magnetic field inhomogeneities with the N4 algorithm presented problems primarily with FLAIR sequences. The hyper-intensity presented in the FLAIR sequence related to the edema was confused frequently with inhomogeneities of the magnetic field, thereby reducing its intensity. In order to overcome this problem we reduced the number of iterations of the algorithm to remove as much inhomogeneities as possible, while keeping the intensities of the lesion. Such solution assumed a non optimal removal of the magnetic field inhomogeneities, but allowed to save the information contained in the lesion area, which becomes more important to the segmentation task. We empirically set a maximum of 10 iterations at each scale of the multi-scale approach of the N4 algorithm.

Several unsupervised classification algorithms were evaluated to assess its pros and cons, ranging from the most restrictive algorithms in terms of class-conditional probabilistic models (K-means and Fuzzy K-means) to more sophisticated models with more degrees of freedom such as GMM or Gauss-HMRF. The last one, also introduces statistical dependencies between adjacent variables of the model, that penalizes neighboring voxels with different labels. Hence, this structured prior aims to model the self similarity of the images, leading the algorithm to a more homogeneous segmentation than the non-structured classification techniques.

Therefore, the less restrictive algorithms were expected to achieve better results based on the hypothesis that these algorithms learn a more flexible model that best fits the data to be classified. Moreover, structured algorithms were also expected to obtain better results based on the hypothesis that these algorithms introduce mechanisms to model the self similarity of the images. Tables 3.2 and 3.3 confirm such hypotheses. Both GMM and Gauss-HMRF rose as the best algorithm tested in almost all the 


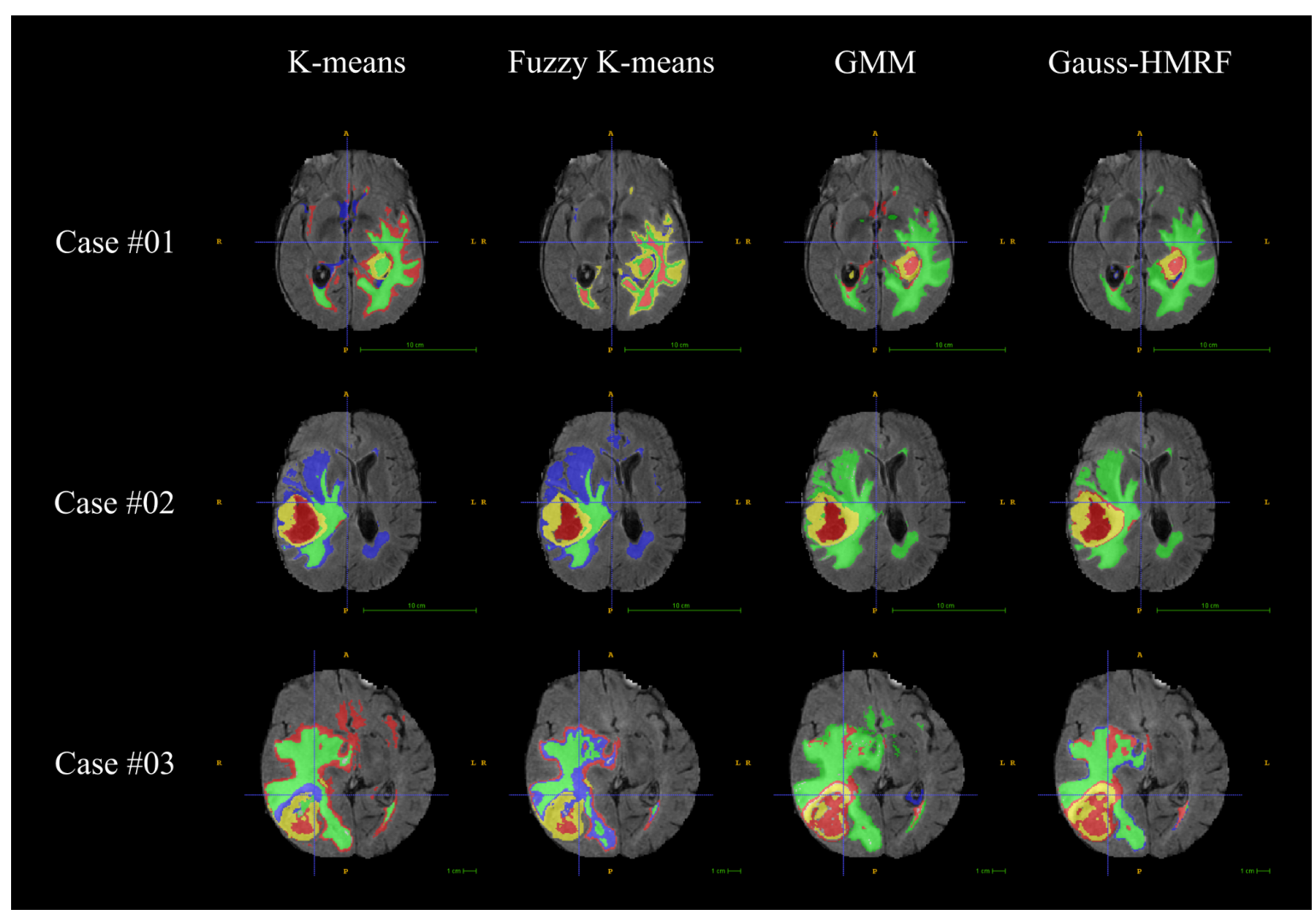

Figure 3.6: Examples of final segmentations of 3 patients of BRATS 2013 dataset computed by the different unsupervised algorithms.

metrics returned by the evaluation web page. Only the results obtained by the GaussHMRF model in the enhancing tumor sub-compartment of the Leaderboard set were not comparable with the other sub-compartments and datasets results. This effect was due to the smoothing prior imposed by the Gauss-HMRF, which was too strong in some cases. We revised the cases that achieved low results in the enhancing tumor sub-compartment and realized that most of them had a large necrotic core with a thin low-brightness enhancing tumor ring. We also revised the K-means++ initializations and realized that the enhancing tumor was partially segmented in some cases but finally lost in the final segmentation due to the hard smoothing prior in the necrotic class. We are currently working on the introduction of different penalizations for the labels, depending on their statistical distribution similarities to avoid this over-smoothing.

It is worth noting that we obtained better results on the Leaderboard set (Table 3.5) than in the Test set (Table 3.4), in contrast with the rest of participants. This effect may have been produced by the fact that the Leaderboard set may include more heterogeneities and differences with respect to the Training set than to the Test set, thereby directly affecting the supervised approaches performance. Unsupervised paradigm avoids this possible overfitting by building a particular model for each patient considering only its own data, therefore achieving better results in the Leaderboard set against most of the supervised approaches evaluated.

In future work, we plan to improve our feature extraction process by analyzing the influence of the texture images in the final segmentations and including more 
sophisticated textures such as the Haralick texture features. Furthermore, we plan to extend our unsupervised methodology to the analysis and segmentation of PWI in combination with anatomical images. The biomarkers obtained from PWI might discover relevant segmentations by adding additional valuable functional information about the tissues. We consider that research efforts should be aligned with quantitative MRI by providing powerful systems that leverage the information contained in these images. 
Chapter 3. Comparative study of unsupervised learning algorithms for glioblastoma segmentation 


\section{Chapter 4}

\section{Non Local Spatially Varying Finite Mixture Models for unsupervised image segmentation}

As stated in the previous chapter, image segmentation is one of the most important core problems in computer vision. It constitutes one of the basic and fundamental steps for automated image understanding since its purpose is to delineate objects in the image with semantic meaning. Innumerable approaches have been proposed in the literature to address this problem, ranging from supervised to unsupervised ML approaches. The latter are indispensable to the image understanding and segmentation task as they provide a robust and reliable solution to all the problems that do not have manually annotated datasets, which ultimately represent the vast majority of real-life image segmentation problems.

Images are structured arrangements of data in which, in addition to the pixel intensities, the location of the pixels provides important information to properly understand its content. Structured learning models capable to properly capture the patterns of local regularity and spatial redundancy of the images have demonstrated their superiority in the image segmentation task.

In this chapter a Bayesian model for unsupervised image segmentation based on a combination of the SVFMMs and the NLM framework is presented. Such model successfully integrates a gauss-markov random field into a classic FMM, to simultaneously codify the idea that neighboring pixels tend to belong to the same semantic object, but preserving the edges and structure of the image. The chapter introduces the mathematical foundations of the model and their estimation via a MAP-EM scheme. We present an evaluation of the performance of the model in a synthetic medical imaging corpus and with a reference dataset of real-world images.

The contents of this chapter were published in the journal publication (Juan-Albarracin et al, 2019b)-thesis contributions C2 and P3. 


\subsection{Introduction}

Unsupervised learning has historically played a key role in the image segmentation task, constituting one of the first paradigms to automatically identify objects and structures in an image (Zhang et al, 2008). Specifically, clustering has gathered most of the efforts in unsupervised image segmentation research. Clustering is the task of finding natural groupings of data within a population, sharing a similar set of properties (Rokach and Maimon, 2005). Many clustering techniques have been proposed in the literature during the past decades (Saxena et al, 2017), ranging from distance based techniques such as partitional clustering or hierarchical clustering; density-based techniques such as DBSCAN (Ester et al, 1996) or Mean Shift (Cheng, 1995); graph based algorithms such as graph-cuts (Boykov et al, 2001); or probabilistic models such as Finite Mixture Models (FMMs)(Pal and Pal, 1993).

Specifically, probabilistic models intend to learn the pdf of an image by means of fitting a multi-parametric statistical model to the data. In particular, FMMs fit a weighted sum of probabilistic distributions, each one representing a component of the image, to capture the heterogeneity nature of the image information. GMMs are the most extended FMMs, being widely employed for image segmentation, as they have proven to successfully capture the complexity of an image (Juan-Albarracín et al, 2015b). Moreover, GMMs can be efficiently estimated by means of MLE via the Expectation-Maximization (EM) algorithm (Dempster et al, 1977).

However, learning from an image has several particularities that must be taken into account. Images are structured arrangements of data in which, in addition to the pixel intensities, the location of these intensities provide important information to properly understand its content. Images show patterns of local regularity and spatial intensity redundancy that enclose the idea that adjacent pixels tend to belong to the same semantic object. Conventional FMMs, by the opposite, do not inherently take into account this information. FMMs make the heavy assumption that data in an image is i.i.d., ignoring the spatial information that has demonstrated to be useful to generate more accurate and realistic models.

To overcome this limitation, several solutions have been proposed in the literature (Blake and Rother, 2011). Most of them rely on the inclusion of a MRF to model the local dependencies between pixels in an image. Specifically, a variant to the FMM called SVFMM was proposed by Sanjay-Gopal and Hebert (1998), which replaces the classics mixing coefficients of the FMM by contextual mixing coefficients for each pixel of the image. This approximation allows to introduce a continuous MRF over these contextual mixing coefficients to incorporate the idea that neighboring pixels tends to share the same intensity properties.

Many variants of MRFs have been proposed in the literature to capture the local information contained in an image. Nikou et al (2007) proposed a family of Gauss-MRFs, successfully achieving better results than the classic FMMs. However, such approximation introduces a local isotropic smoothing over the contextual mixing coefficients, that ignores the presence of edges in the image. Therefore, the contextual mixing coefficients estimated under the Gauss-MRF approximation are iteratively smoothed, yielding prior probability maps that lose the information of image edges. Sfikas et al (2008) proposed a t-Student MRF that allowed to regulate the smoothing between pix- 
els in an edge. However, this approximation introduces new parameters to be estimated in the model, yielding a non closed-form analytic solution for it.

In this chapter a fully Bayesian SVFMM model, called NLSVFMM, that combines the SVFMM framework with the NLM filtering schema is proposed. The model has 2 variants: the pixel-wise version (NLv-SVFMM) and the patch-wise version (NLpSVFMM). The proposed model introduces a Gauss-MRF weighted by the probabilistic NLM function proposed by Wu et al (2013) to adaptively adjust the spatial regularization depending on the structure of the image. Such approximation avoids the introduction of new parameters, reducing the degrees of freedom of the model and the number of samples required for a reliable estimation of the parameters.

\subsection{Background on Spatially Varying Finite Mix- ture Models}

The SVFMM is a modification of the classic FMM, focused mainly on imaging data, in which the coefficients of the mixture are related to each other through a structured graph that defines the statistical dependencies between them. SVFMMs are thoroughly introduced in section 2.4, however, in order for this chapter to be self-contained, a short remainder will be made.

Let $X=\left(\mathbf{x}^{1}, \ldots, \mathbf{x}^{N}\right)$ a set of observations corresponding to the pixels of an image, where $\mathbf{x}^{i} \in \mathbb{R}^{D}$ and represents a vector of $D$ features for the $i^{t h}$ pixel. The SVFMM is defined as:

$$
p(X \mid \Theta, \Pi)=\prod_{i=1}^{N} \sum_{j=1}^{K} \pi_{j}^{i} \phi\left(\mathbf{x}^{i} ; \Theta_{j}\right)
$$

where $\phi\left(\mathbf{x}^{i} \mid \Theta_{j}\right)$ is a pdf used to model the data (typically a Normal or t-Student distribution) and $\Theta=\left\{\Theta_{1}, \ldots, \Theta_{K}, \pi^{1}, \ldots, \pi^{N}\right\}$ the set of parameters of the model, with $\Pi=\left\{\pi^{1}, \ldots, \pi^{N}\right\}$ called the contextual mixing coefficients, which must comply with:

$$
\forall \pi^{i}, \quad 0 \leq \pi_{j}^{i} \leq 1, \quad \sum_{j=1}^{K} \pi_{j}^{i}=1
$$

A MAP estimate of $(\Theta, \Pi)$ is typically conducted to impose a proper prior over $\Pi$ to introduce the idea that neighboring pixels in an image tend to belong to the same semantic object.

$$
(\hat{\Theta}, \hat{\Pi})_{M A P}=\underset{(\Theta, \Pi)}{\arg \max } \log p(X \mid \Theta, \Pi)+\log p(\Pi)
$$

Several variants of $p(\Pi)$ have been proposed in the literature. Specifically, the DCAGMRF, introduced in equation 2.65, has proven to successfully capture the local redundancy and spatial regularity inherent in images, regularizing many ill-posed inverse problems with successful results (please refer to section 2.4.2 for a detailed description of the DCAGMRF prior). 
As stated in section 2.4.2, inference on SVFMMs is not analytically tractable. Numerical optimization methods are therefore required to estimate $(\Theta, \Pi)$ in a tractable manner. Typically, a MAP-EM algorithm is used to iteratively find the updates of the model parameters, based on the conditional expectation of the log-likelihood function, used to guide the estimation procedure. Nevertheless, no closed-form solution can be obtained for $\pi^{i}$ when considering the restriction stated in 4.2. To overcome this limitation, an alternative solution is to consider $\Pi$ as a random variable governed by a DCM distribution. Nikou et al (2010) demonstrated that, following such approach, $\pi_{j}^{i}$ can be computed as:

$$
\pi_{j}^{i}=\frac{\alpha_{j}^{i}}{\sum_{k=1}^{K} \alpha_{k}^{i}}
$$

with $\alpha^{i}$ the parameters of the Dirichlet distribution. It is easy to see that such approach always guarantees that $\sum_{j} \pi_{j}^{i}=1 \quad \forall i$, hence satisfying the condition settled in 4.2 . In addition, $\alpha^{i}$ parameters only require to satisfy $\alpha_{j}^{i}>0 \forall i, j$, making easier their optimization.

Therefore, following this DCM-SVFMM approach, the DCAGMRF density can be imposed over $A=\left\{\alpha^{1}, \ldots, \alpha^{N}\right\}$ (instead of over $\Pi$ ), to enforce the desired local regularity:

$$
p(A)=\prod_{i=1}^{N} \prod_{j=1}^{K} \prod_{d=1}^{D} \prod_{m \in \mathcal{M}_{d}^{i}} \frac{1}{\sqrt{2 \pi \beta_{j, d}^{2}}} \exp \left(-\frac{\left(\alpha^{i}-\alpha_{j}^{m}\right)^{2}}{2 \beta_{j, d}^{2}}\right)
$$

where sub-index $d$ refers to the different spatial adjacency directions (i.e. horizontals, verticals or diagonals), and $\mathcal{M}_{d}^{i}$ indicates the set of neighbors of the $i^{\text {th }}$ pixel that lies in the $d^{\text {th }}$ spatial direction.

The complete step-by-step estimation of the parameters of the DCM-SVFMM via the EM algorithm is described in section 2.4.3. We encourage the reader to review this section for an in-depth explanation of the estimation procedure of the DCM-SVFMMs.

\subsection{Background on Probabilistic Non Local Means}

The NLM filter (Buades et al, 2005a) proposes a schema for image filtering where pixels are restored by a weighted sum of similar neighbor patches.

The core of NLM schema is the weight function that relates neighboring patches, which has taken a lot of variants in the literature. Specially, Wu et al (2013) derived the probabilistic version of the NLM algorithm and its associated probabilistic weighting function.

In order to relate the description of the probabilistic NLM with the SVFMM background, let's consider $d_{j, d}^{i, m}$ as the distance between a pair of adjacent Dirichlet parameters in the form: 


$$
d_{j, d}^{i, m}=\frac{\left(\alpha_{j}^{i}-\alpha_{j}^{m}\right)^{2}}{2 \beta_{j, d}^{2}}
$$

Assuming that local differences are i.i.d., we have $d_{j, d}^{i, m} \sim \chi^{2}(1)$. For a patch-based version of the algorithm, the distance between two patches centered at $i^{\text {th }}$ and $m^{\text {th }}$ locations is defined as:

$$
D_{j, d}^{i, m}=\sum_{k \in \mathbb{P}} d_{j, d}^{i+k, m+k}
$$

where $\mathbb{P}$ is the set of offsets that define a local patch around a given pixel. If patches are completely disjoint, then $D_{j, d}^{i, m} \sim \chi^{2}(|\mathbb{P}|)$, however, in most cases, overlapping occurs between patches, so the i.i.d. assumption does not hold. In such cases, an approximation to the sum of a set of correlated $\chi^{2}$ distributions can be computed as:

$$
D_{j, d}^{i, m} \sim \gamma_{m} \chi^{2}\left(\eta_{m}\right)
$$

where

$$
\begin{aligned}
\gamma_{m} & =\operatorname{var}\left[D_{j, d}^{i, m}\right] / 2 \mathrm{E}\left[D_{j, d}^{i, m}\right] \\
\eta_{m} & =\mathrm{E}\left[D_{j, d}^{i, m}\right] / \gamma_{m}
\end{aligned}
$$

and

$$
\begin{aligned}
\mathrm{E}\left[D_{j, d}^{i, m}\right] & =|\mathbb{P}| \\
\operatorname{var}\left[D_{j, d}^{i, m}\right] & =2|\mathbb{P}|+\left|\mathbb{O}^{i, m}\right|
\end{aligned}
$$

with $\mathbb{O}^{i, m}$ the set of overlapping pixels between the patches centered at $i^{\text {th }}$ and $m^{\text {th }}$ pixels.

Hence, the weight function $u_{j, d}^{i, m}$ proposed in the probabilistic NLM approach, associated to the SVFMM framework, is defined as

$$
u_{j, d}^{i, m}=\chi^{2}\left(D_{j, d}^{i, m} / \gamma_{m} \mid \eta_{m}\right)=\frac{\left(D_{j, d}^{i, m} / \gamma_{m}\right)^{\left(\eta_{m} / 2\right)-1} \exp \left(-D_{j, d}^{i, m} / 2 \gamma_{m}\right)}{2^{\eta_{m} / 2} \Gamma\left(\eta_{m} / 2\right)}
$$

\subsection{The Non Local Spatially Variant Finite Mix- ture Model}

One of the main drawbacks of the SVFMM-based models is that they enforce a local smoothness on the contextual mixing coefficients (or Dirichlet parameters) without taking into account the structure of the image. In other words, the SVFMM iteratively applies an isotropic local Gaussian smoothing to these parameters, leading to a oversmoothed prior probability map that losses the information of the edges and structures in the image. 
To overcome this limitation, Sfikas et al (2008) proposed a variant of the SVFMM where local differences between Dirichlet parameters follow a t-Student distribution. Such an approach was intended to exploit the heavy-tailed nature of the t-Student distribution, to perform a robust estimation of the Dirichlet coefficients when edges and structures are present in their local neighborhoods.

$$
\alpha_{j}^{i}-\alpha_{j}^{m} \sim \mathcal{S} t\left(0, \beta_{j, d}^{2}, \nu_{j}\right)
$$

Following the Bishop's development in (Bishop, 2006), a $\mathcal{S} t$ distribution can be expressed as:

$$
\begin{aligned}
\alpha_{j}^{i}-\alpha_{j}^{m} & \sim \mathcal{N}\left(0, \beta_{j, d}^{2} / g_{j, d}^{i, m}\right) \\
g_{j, d}^{i, m} & \sim \mathcal{G}\left(\nu_{j, d} / 2, \nu_{j, d} / 2\right)
\end{aligned}
$$

This model introduces a new set of latent variables $g_{j, d}^{i, m}$, whose posterior density should be estimated at the E-step, and a new set of parameters $\nu_{j, d}$, with non closedform analytic estimation. Therefore, numerical optimization methods such as NewtonRaphson or Brent's methods should be employed to estimate $\nu_{j, d}$.

In this sense, and similar in spirits than the t-Student model, in this chapter we propose the NLSVFMM as a modification of the Sfikas' t-Student model, by replacing the $g_{j, d}^{i, m}$ random variable by the probabilistic NLM $u_{j, d}^{i, m}$ weight. Therefore, we propose to reformulate the local differences between contextual Dirichlet parameters to follow a NLM-related distribution, denoted by:

$$
\begin{aligned}
\alpha_{j}^{i}-\alpha_{j}^{m} & \sim \mathcal{N}\left(0, \beta_{j, d}^{2} / \chi^{2}\left(D_{j, d}^{i, m} / \gamma_{m} \mid \eta_{m}\right)\right) \\
D_{j, d}^{i, m} & \sim \gamma_{m} \chi^{2}\left(\eta_{m}\right)
\end{aligned}
$$

with $D_{j, d}^{i, m}$ being latent variables of the model.

Following the conventional EM scheme, the posterior densities of $D_{j, d}^{i, m}$ should be calculated at the E-step. However, this leads to a different calculation of $D_{j, d}^{i, m}$ than the proposed by Wu et al (2013) (see Section 4.3). Therefore, in order to preserve the use of the original NLM weights, we will follow a Variational EM approach (Neal and Hinton, 1999; Bishop, 2006). The Variational EM framework introduces the concept of partial E-step, in which a functor of the latent variables can be used when the posterior densities of these variables cannot be calculated, or when it is desirable to calculate them differently for reasons of efficiency or performance. As demonstrated by Neal and Hinton (1999), such functor can take any form as long as the log-likelihood function is increased at each iteration, effectively driving the model to a local optimum of the function, and hence to an optimum of the parameters of the model. Therefore, following this framework, the $D_{j, d}^{i, m}$ latent variables are estimated at the E-step as the standard quantitative Chi-squared test proposed by Wu et al (2013):

$$
D_{j, d}^{i, m}=\sum_{k \in \mathbb{P}} \frac{\left(\alpha_{j}^{i+k}-\alpha_{j}^{m+k}\right)^{2}}{2 \beta_{j, d}^{2}}
$$




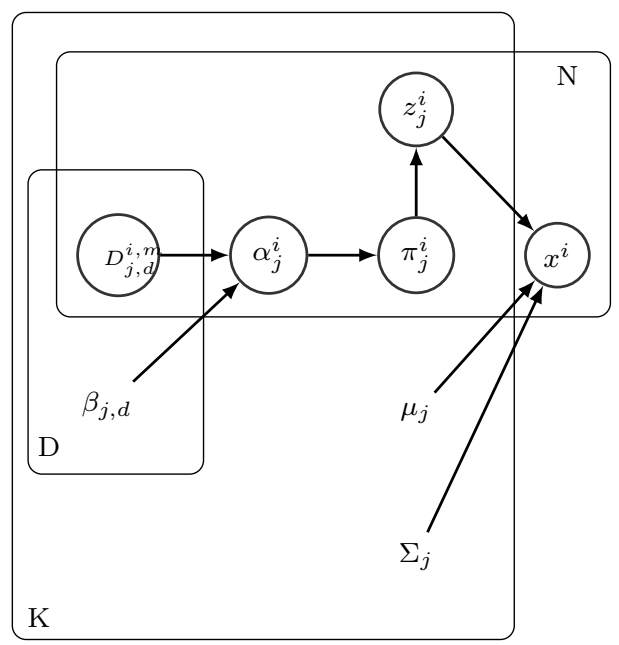

Figure 4.1: Graphical model for the non-local spatially varying finite mixture model. Superscripts $i, m \in[1, N]$ denote pixel indexes, subscript $j \in[1, K]$ denotes mixture component and subscript $d \in[1, D]$ denotes neighborhood direction.

Once these latent variables are estimated, the $u_{j, d}^{i, m}$ weights are calculated at the M-step following $u_{j, d}^{i, m}=\chi^{2}\left(D_{j, d}^{i, m} / \gamma_{m} \mid \eta_{m}\right)$. Since $u_{j, d}^{i, m}$ depends on both the $i^{t h}$ and $m^{\text {th }}$ observations, this model specifies a different instance of a Gaussian distribution for each $\left(\alpha_{j}^{i}-\alpha_{j}^{m}\right)$ pair of contextual Dirichlet coefficients in the MRF. This allows $u_{j, d}^{i, m}$ to regulate the variance of the corresponding Gaussian between the $i^{\text {th }}$ and $m^{t h}$ observations, if an edge or an homogeneous area is detected at this location. Thus, as $u_{j, d}^{i, m}$ increases, the Gaussian distribution for the corresponding pair shrinks around zero imposing a hard smoothing between the observations. On the contrary, as $u_{j, d}^{i, m}$ decreases, the variance of the Gaussian distributions increases producing a lower pdf value that prevents the smooth.

This approximation avoids the introduction of new parameters since $\eta_{m}$ and $\gamma_{m}$ are completely known once $i$ and $m$ are fixed. Therefore, no numerical approximate methods are required, simplifying the model and reducing its degrees of freedom and the number of samples required for its statistically reliable estimation.

The graphical model of the NL-SVFMM is shown in Figure 4.1.

Imposing the DCAGMRF prior to the proposed NLSVFMM model, the new density for $p(A)$ becomes

$$
p(A)=\prod_{i=1}^{N} \prod_{j=1}^{K} \prod_{d=1}^{D} \prod_{m \in \mathcal{M}_{d}^{i}} \frac{1}{\sqrt{2 \pi \beta_{j, d}^{2} / u_{j, d}^{i, m}}} \exp \left(-\frac{\left(\alpha^{i}-\alpha_{j}^{m}\right)^{2} u_{j, d}^{i, m}}{2 \beta_{j, d}^{2}}\right)
$$

which setting $\partial Q / \partial \alpha_{j}^{i}$ yields a third degree equation of the form:

$$
\left(\alpha_{j}^{i(t+1)}\right)^{3}+\left(\alpha_{j}^{i(t+1)}\right)^{2}\left(A_{-j}^{i}-\frac{\widehat{C}_{j}^{i}}{\widehat{B}_{j}^{i}}\right)-\left(\alpha_{j}^{i(t+1)}\right)\left(\frac{A_{-j}^{i} \widehat{C}_{j}^{i}}{\widehat{B}_{j}^{i}}\right)-\frac{z_{j}^{i} A_{-j}^{i}}{2 \widehat{B}_{j}^{i}}=0
$$

where 


$$
\begin{aligned}
A_{-j}^{i} & =\sum_{\substack{k=1 \\
k \neq j}}^{K} \alpha_{k}^{i(t)} \\
\widehat{B}_{j}^{i} & =\sum_{d=1}^{D} \frac{\sum_{m \in \mathcal{M}_{d}^{i}} u_{j, d}^{i, m}(t)}{\beta_{j, d}^{2}{ }^{(t)}} \\
\widehat{C}_{j}^{i} & =\sum_{d=1}^{D} \frac{\sum_{m \in \mathcal{M}_{d}^{i}} \alpha_{j}^{m(t)} u_{j, d}^{i, m}(t)}{\beta_{j, d}^{2(t)}}
\end{aligned}
$$

Likewise the conventional DCM-SVFMM, it can be demonstrated that, under polynomial theory, there is always a real non negative solution that satisfies $\alpha_{j}^{i} \geq 0$. The Vieta's method is used to algebraically obtain the roots of the proposed third degree equation.

Finally, $\beta_{j, d}^{2}$ is estimated as:

$$
\beta_{j, d}^{2(t+1)}=\frac{1}{N} \sum_{i=1}^{N} \sum_{m \in \mathcal{M}_{d}^{i}} \frac{\left(\alpha_{j}^{i^{(t+1)}}-\alpha_{j}^{m(t+1)}\right)^{2} u_{j, d}^{i, m}(t)}{\left|\mathcal{M}_{d}^{i}\right|}
$$

Hereafter, the pixel-wise $\chi^{2}(1)$ version of the proposed NLSVFMM will be referred as NLv-SVFMM, while the patch-wise $\chi^{2}\left(\eta_{m}\right)$ will be referred as NLp-SVFMM.

\subsection{Experimental results}

Both variants of the proposed NLSVFMM algorithm have been evaluated in a simulated and a real-world scenario. First an evaluation on a synthetic high grade glioma dataset of the BRATS 2013 Challenge was conducted (Menze et al, 2015). Next, and evaluation over the 300 real-world images of the Berkeley Segmentation dataset was performed (Martin et al, 2001). We have compared our proposed NLv- and NLpSVFMM model with the conventional FMM, the SVFMM and the $\mathcal{S}$ t-SVFMM. For the spatially varying algorithms we have employed the DCM Bayesian approximation and the DCAGMRF prior specified in 4.15. All algorithms in all experiments were initialized with a deterministic version of K-means++ (Arthur and Vassilvitskii, 2007) to ensure a fair comparative. In addition, results on the behavior of the weighting functions and weighting maps of the NLSVFMM and t-Student model are shown. The evaluation is presented below.

\subsubsection{Evaluation on the synthetic BRATS 2013 high grade glioma dataset}

The BRATS 2013 high grade glioma synthetic dataset is composed of 25 cases, each one segmented into 7 labels: 1) WM, 2) GM, 3) CSF, 4) peripheral edema (ED), 
5) tumor core (split into enhancing tumor (5.1) and necrotic core (5.2)) (TC) and 6) vessels (VS). For each voxel, intensities on pre- and post-gadolinium $\mathrm{T}_{1}$-weighted, $\mathrm{T}_{2}$-weighted and FLAIR MRI sequences were employed for the segmentation.

Figure 4.2 compares the behavior of the weighting functions $G=\left\{g_{j, d}^{i, m}\right\}$ for the $\mathcal{S}$ tSVFMM model and $U=\left\{u_{j, d}^{i, m}\right\}$ for the NLv-SVFMM model (NLp-SVFMM weighting function is not depicted because is not numerically comparable to the $\mathcal{S}$ t and NLvSVFMM models).

As figure shows, $U$ function behaves more aggressive for differences between observations than the $\mathcal{S}$ t-SVFMM, hence yielding more dichotomous weighting maps (see Figure 4.3). For the shake of simplicity, each pixel of each picture of Figure 4.3 represent $\sum_{d=1}^{D} \sum_{m \in \mathcal{M}^{i}} \lambda_{j, d}^{i, m}$, with $\lambda=g$ for $\mathcal{S}$ t-SVFMM and $\lambda=u$ for NLv- or NLpSVFMM models respectively. The weighting maps of Figure 4.3 demonstrates that the NLSVFMM-based algorithms better differentiates among tissues than than the $\mathcal{S t - S V F M M . ~}$

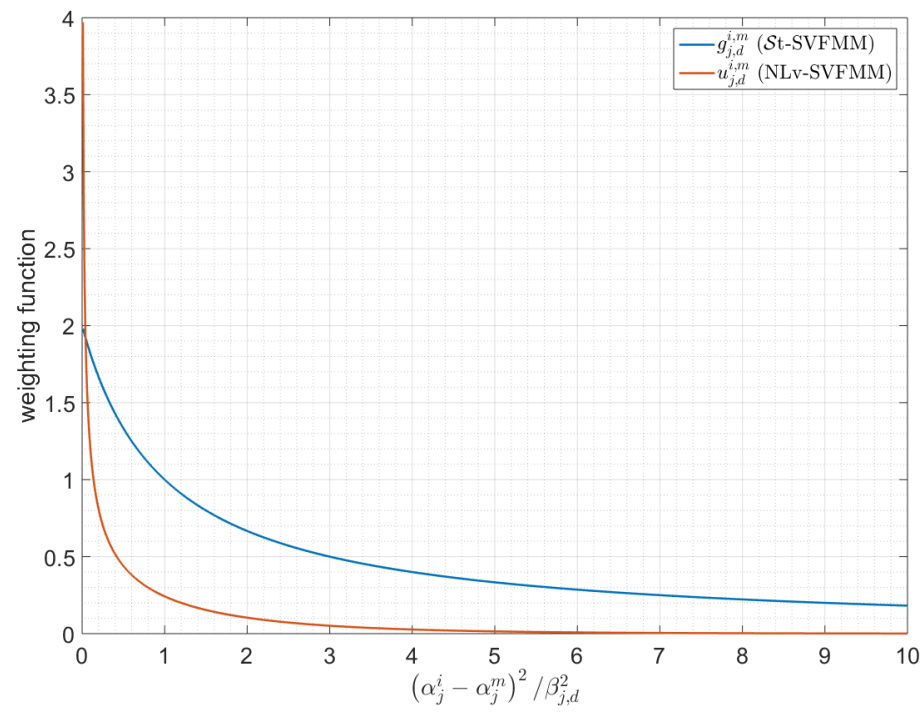

Figure 4.2: Comparison between the behavior of the weighting functions $G=\left\{g_{j, d}^{i, m}\right\}$ for the $\mathcal{S}$ tSVFMM model and $U=\left\{u_{j, d}^{i, m}\right\}$ for the NLv-SVFMM (NLp-SVFMM weighting function is not depicted because is not numerically comparable to the $\mathcal{S}$ t and NLv-SVFMM functions).

Table 4.1 and Figure 4.4 show the superiority of the proposed NL-SVFMM (in both variants) to generate higher confidence prior probability maps for each component. An example of the contextual mixing coefficient maps for the HG0014 case of the BRATS 2013 dataset and its associated mixing coefficient values for different pixels obtained by each method is shown. In almost all evaluations, the NLp-SVFMM version achieves the best results, indicating that the patch-based probabilistic NLM weighting function better captures the local similarities in the images.

Table 4.2 shows the Dice coefficients obtained for the evaluation based on the BRATS 2013 dataset. Consistently with previous results, the NLp-SVFMM variant achieves the best results in terms of segmentations based on the maximization of the posterior probabilities (Bayes minimum classification error). An improvement of 


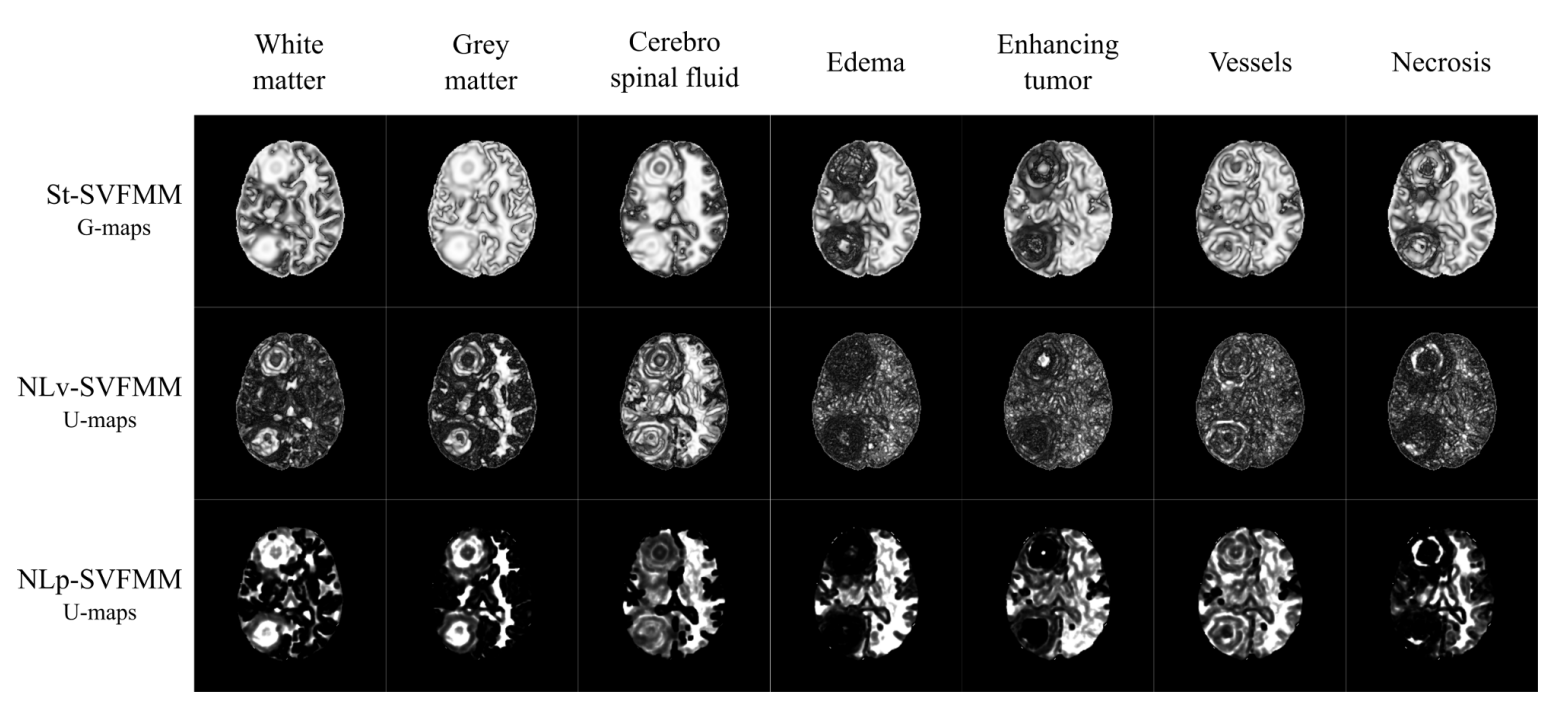

Figure 4.3: Comparison between $G$ maps of the St-SVFMM and $U$ maps for the NLv- and NLpSVFMM models for a case of the BRATS 2013 dataset. Each pixel $i$ of the images represent $\sum_{d=1}^{D} \sum_{m \in \mathcal{M}^{i}} \lambda_{j, d}^{i, m}$, with $\lambda=g$ or $\lambda=u$ for $\mathcal{S}$ t-SVFMM and NLv- or NLp-SVFMM models respectively.

about 3 points in Dice is obtained when comparing the NLp-SVFMM with the standard SVFMM and more than 1 point in Dice with respect to the $\mathcal{S}$ t-SVFMM, thanks to the proposed prior density. Moreover, in order to explore the capabilities of the proposed prior densities to yield accurate segmentations, we have also computed the Dice coefficients for the segmentations based only on the maximization of the prior probability maps generated by each method. As Table 4.2 shows, the NLv-SVFMM method, followed by the NLp-SVFMM, achieves the best results. Of course the Dice coefficients are significantly low because the segmentations do not take into account the pixel intensities. However, the aim is to evaluate which method better captures the local similarities in the images, hence producing more accurate prior information about the different segments in the image.

\subsubsection{Evaluation on the Berkeley Segmentation Dataset}

In addition to the synthetic evaluation, we have assessed the performance of the proposed model with the 300 real-world images of the Berkeley Segmentation dataset. In our experimentation, we have employed a 3-dimensional feature vector to represent each each pixel of the images, comprising the 3 channels of the $\mathrm{L}^{*} \mathrm{a} * \mathrm{~b}$ color space. We also applied a local median smoothing to each channel using a $5 \times 5$ window centered at each pixel. We have evaluated the performance of each algorithm for different values of $K=\{3,5,7,10,15,20\}$.

Table 4.3 shows the results for the evaluation of the 300 images of the Berkeley dataset. Rand Index (RI) is employed to measure the degree of concordance between the automated segmentation and the manual segmentations. Our experiments show that the proposed methods perform favorably in terms of RI to the other approaches in almost all situations. The NLv-SVFMM performs comparable to the $\mathcal{S}$ t variant in most of cases, achieving very similar results. However, the NLv-SVFMM requires less 
Table 4.1: Contextual mixing coefficients for different voxels of the HG0014 case of the BRATS 2013 challenge. Voxels correspond to coordinates $a=(151,127,85), b=(167,75,85), c=$ $(151,152,85), d=(97,89,85), e=(117,62,85), f=(110,71,85)$ and $f=(128,99,85)$

\begin{tabular}{lcccc}
\hline & SVFMM & St-SVFMM & NLv-SVFMM & NLp-SVFMM \\
$\pi_{W M}^{a}$ & 0.457 & 0.486 & 0.481 & $\mathbf{0 . 4 9 9}$ \\
$\pi_{G M}^{b}$ & 0.296 & 0.429 & 0.497 & $\mathbf{0 . 6 0 6}$ \\
$\pi_{C S F}^{c}$ & 0.253 & 0.426 & 0.446 & $\mathbf{0 . 4 4 7}$ \\
$\pi_{E D}^{d}$ & 0.294 & 0.374 & 0.394 & $\mathbf{0 . 4 1 1}$ \\
$\pi_{E T}^{e}$ & 0.248 & 0.442 & $\mathbf{0 . 4 5 3}$ & 0.381 \\
$\pi_{N C}^{f}$ & 0.267 & 0.291 & 0.285 & $\mathbf{0 . 3 6 1}$ \\
$\pi_{V S}^{g}$ & 0.118 & 0.305 & $\mathbf{0 . 3 5 5}$ & 0.265 \\
\hline
\end{tabular}

Table 4.2: Results on the Dice coefficient over the 25 synthetic high-grade gliomas of the BRATS 2013 dataset for each algorithm evaluated. Segmentations based on the maximization of the posterior and prior probabilities are shown.

\begin{tabular}{ccccccccccccccc}
\hline \multirow{2}{*}{ Segmentation } & \multicolumn{3}{c}{ SVFMM } & \multicolumn{4}{c}{ St-SVFMM } & \multicolumn{3}{c}{ NLv-SVFMM } & \multicolumn{3}{c}{ NLp-SVFMM } \\
& Mean & Median & Std. & Mean & Median & Std. & Mean & Median & Std. & Mean & Median & Std. \\
Posterior & 0.7766 & 0.7680 & 0.0368 & 0.7912 & 0.7817 & 0.0384 & 0.7988 & 0.7833 & 0.0397 & $\mathbf{0 . 8 0 4 4}$ & $\mathbf{0 . 7 9 3 6}$ & 0.0351 \\
Prior & 0.2231 & 0.2247 & 0.0092 & 0.2467 & 0.2461 & 0.0103 & $\mathbf{0 . 2 5 7 6}$ & $\mathbf{0 . 2 5 7 8}$ & 0.0113 & 0.2494 & 0.2495 & 0.0106 \\
\hline
\end{tabular}

parameters, hence reducing the degrees of freedom of the model. Nevertheless, the NLp-SVFMM method achieves, both in average and median cases, the best results in most cases. Only in the $K=3$ case (the simplest segmentation), the SVFMM method outperforms the rest of the models. However, as segmentation complexity increases, the models including edge preserving priors performs better in all cases.

Table 4.3: Results on the RI over the 300 images of the Berkeley dataset for each algorithm evaluated.

\begin{tabular}{ccccccccccccccccccccccccc}
\hline & \multicolumn{3}{c}{ SVFMM } & \multicolumn{4}{c}{ St-SVFMM } & \multicolumn{4}{c}{ NLv-SVFMM } & \multicolumn{4}{c}{ NLp-SVFMM } \\
K & Mean & Median & Std. & K & Mean & Median & Std. & K & Mean & Median & Std. & K & Mean & Median & Std. \\
3 & $\mathbf{0 . 6 9 5 2}$ & $\mathbf{0 . 6 9 1 5}$ & 0.0986 & 3 & 0.6941 & 0.6891 & 0.0988 & 3 & 0.6940 & 0.6891 & 0.0988 & 3 & 0.6944 & 0.6897 & 0.0987 \\
5 & 0.7274 & 0.7478 & 0.1086 & 5 & 0.7284 & $\mathbf{0 . 7 4 8 2}$ & 0.1086 & 5 & 0.7284 & $\mathbf{0 . 7 4 8 2}$ & 0.1085 & 5 & $\mathbf{0 . 7 2 8 8}$ & 0.7480 & 0.1086 \\
7 & 0.7283 & 0.7585 & 0.1208 & 7 & 0.7312 & 0.7596 & 0.1207 & 7 & 0.7313 & 0.7597 & 0.1206 & 7 & $\mathbf{0 . 7 3 1 6}$ & $\mathbf{0 . 7 5 9 9}$ & 0.1207 \\
10 & 0.7250 & 0.7618 & 0.1335 & 10 & 0.7281 & 0.7632 & 0.1333 & 10 & 0.7283 & 0.7634 & 0.1334 & 10 & $\mathbf{0 . 7 2 8 8}$ & $\mathbf{0 . 7 6 3 9}$ & 0.1334 \\
15 & 0.7184 & 0.7585 & 0.1431 & 15 & 0.7215 & 0.7594 & 0.1428 & 15 & 0.7214 & 0.7595 & 0.1428 & 15 & $\mathbf{0 . 7 2 2 1}$ & $\mathbf{0 . 7 6 1 2}$ & 0.1429 \\
20 & 0.7136 & 0.7495 & 0.1479 & 20 & 0.7161 & 0.7538 & 0.1478 & 20 & 0.7162 & 0.7538 & 0.1478 & 20 & $\mathbf{0 . 7 1 6 6}$ & $\mathbf{0 . 7 5 4 5}$ & 0.1479 \\
\hline
\end{tabular}

Figure 4.5 shows several examples of segmentations of images of the Berkeley dataset obtained with the NLp-SVFMM method. 
Chapter 4. Non Local Spatially Varying Finite Mixture Models for unsupervised image segmentation

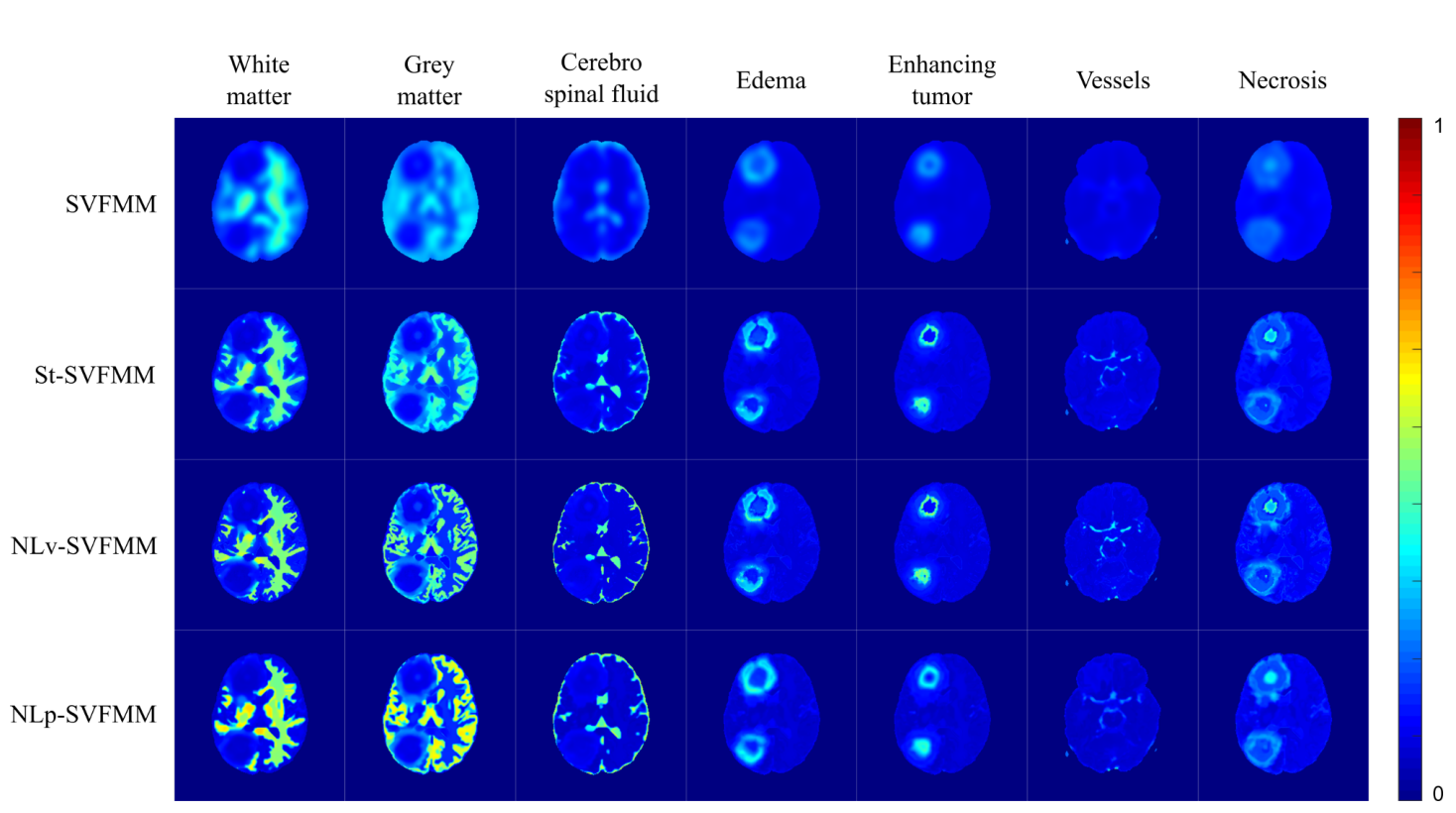

Figure 4.4: Example of contextual mixing coefficient maps for a case of BRATS 2013, for each label of the segmentation.

\subsubsection{Evaluation of computational time requirements}

Additionally, a comparison in terms of the computational time required by each method has been performed. Table 4.4 shows the average times (in seconds) and the std. deviation of each method evaluated in the Berkeley 300 dataset for different number of segments calculated in the images.

Table 4.4: Average and std. deviation time comparison (in seconds) for each algorithm evaluated in the study on the Berkeley 300 dataset for each number of segments computed in the images.

\begin{tabular}{ccccc}
\hline K & SVFMM & St-SVFMM & NLv-SVFMM & NLp-SVFMM \\
3 & $0.91 \pm 0.04$ & $1.48 \pm 0.06$ & $1.73 \pm 0.06$ & $2.01 \pm 0.09$ \\
5 & $1.39 \pm 0.08$ & $2.27 \pm 0.12$ & $2.56 \pm 0.13$ & $2.99 \pm 0.13$ \\
7 & $1.87 \pm 0.08$ & $3.05 \pm 0.15$ & $3.37 \pm 0.14$ & $3.98 \pm 0.16$ \\
10 & $2.69 \pm 0.14$ & $4.40 \pm 0.20$ & $4.92 \pm 0.21$ & $5.75 \pm 0.17$ \\
15 & $3.91 \pm 0.17$ & $6.32 \pm 0.22$ & $6.96 \pm 0.26$ & $8.23 \pm 0.30$ \\
20 & $5.18 \pm 0.47$ & $8.39 \pm 0.75$ & $9.27 \pm 0.82$ & $10.92 \pm 0.96$ \\
\hline
\end{tabular}

As expected, the SVFMM is the fastest method since it doesn't carry the extra computation of the weights for constrain the $\beta_{j, d}^{2}$ variances. It should be noted that only the NLv-SVFMM and the $\mathcal{S}$ t-SVFMM are directly comparable since both perform the calculation of the $u$ and $g$ weights respectively, and those weights are computed pixel-wise. It can be seen that both methods perform very similar, with no significant difference between them. Although the $\mathcal{S}$ t-SVFMM model requires a numerical iterative approximation of the $\nu_{j, d}$ parameters, which is often a slow procedure, the 

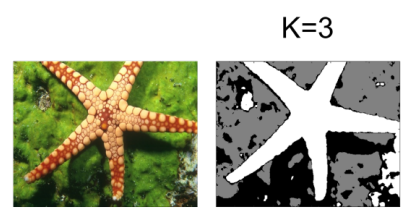

$\mathrm{K}=5$

$\mathrm{K}=7$

$\mathrm{K}=10$

$\mathrm{K}=15$

$\mathrm{K}=20$
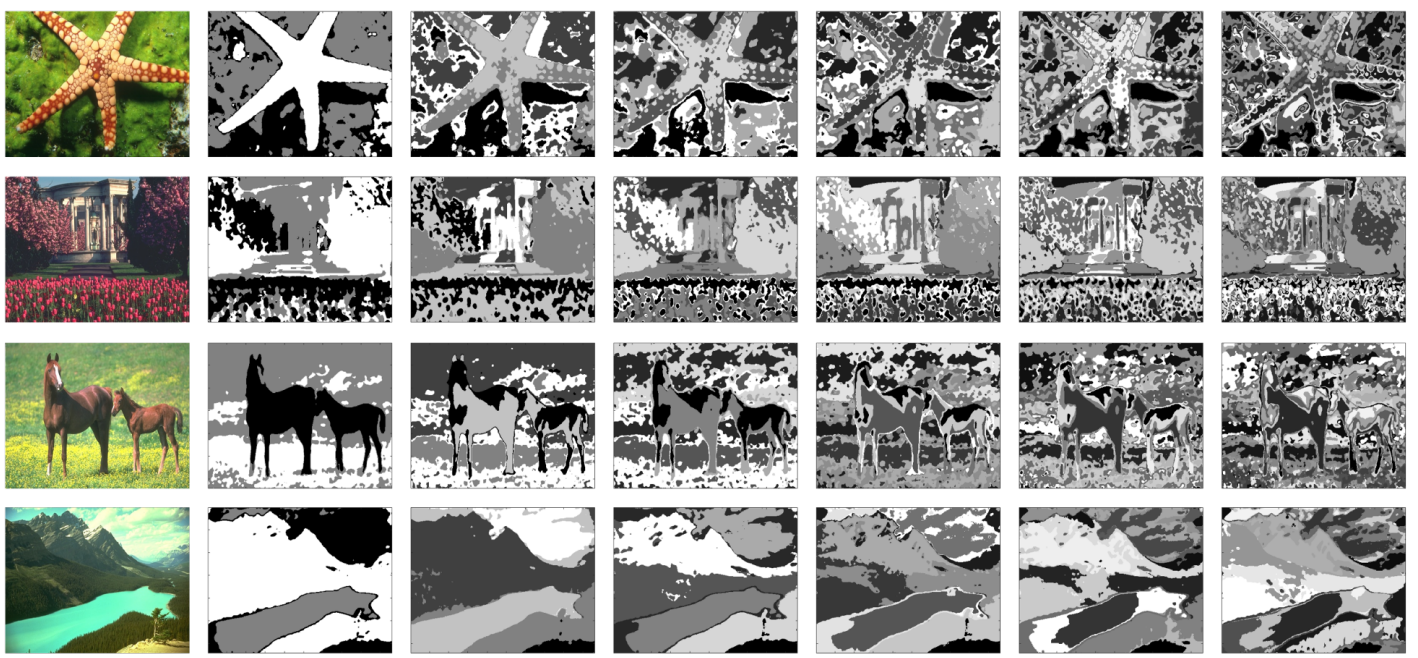

Figure 4.5: Example of segmentation maps for $K \in\{3,5,7,10,15,20\}$ obtained with the NLp-SVFMM for 4 images of the Berkeley dataset.

complexity in the computation of the $g_{j, d}^{i, m}$ weights is lighter than the $u_{j, d}^{i, m}$ weights. That is the reason why the NLv-SVFMM is a bit slower than the $\mathcal{S}$ t-SVFMM. The calculation of $u_{j, d}^{i, m}$ weights requires the computation of $N K D\left|\mathcal{M}_{d}\right| \chi^{2}\left(\eta_{m}\right)$ pdf values, which ultimately equals or even slightly increases the computational time with respect the $g$ weights. The NLp-SVFMM performs the best in terms of Dice and RI scores, but also requires more time to compute the segmentation since it carries the extra computation of the patch-based similarity.

\subsection{Discussion}

In this study we have proposed a new unsupervised image clustering algorithm that successfully merges the SVFMM framework with the well-known NLM filtering scheme. The main advantage of this algorithm is the proposed new MRF density over the contextual mixing proportions, which enforces local smoothness while preserving edges and the structure of the image. This MRF improves the previously proposed $\mathcal{S}$ tMRF both in performance and in complexity of the model by reducing the number of parameters to be estimated. Experimental results demonstrated the superiority of the proposed method with respect to previous state-of-the-art algorithms proposed in the literature when evaluated in a public reference dataset.

As figure 4.2 shows, the proposed probabilistic NLM weighting function behaves more aggressive in the NLSVFMM than its analogous in the $\mathcal{S}$ t-SVFMM. This shrinks or widens the covariance matrices more dramatically when there are differences (even small ones) between adjacent contextual mixing coefficients, leading to a sharper prior density. This behavior tends to estimate more radical prior maps, which hypothetically should provide stronger information during the learning and inference of the model. On the contrary, uniform flat prior probability maps rapidly fade into an uninformative 
element in the model. Figure 4.4 and table 4.1 corroborates this hypothesis. In figure 4.4 , it can be seen that the prior probability maps obtained by the NLSVFMM variants achieve the highest degrees of confidence for the different labels among all models compared. Table 4.1 compares the contextual mixing coefficient values obtained by each method for different voxels corresponding to different tissues of a BRATS case. As it can be seen, the highest values are always obtained by the proposed NLSVFMM variants, leading to a highest degree of confidence about the presence of a specific tissue.

Results on the BRATS synthetic dataset allowed to evaluate the performance of the methods in a controlled environment where the number of labels existing in the data is known. This enabled us to directly measure the impact of the proposed NLM-based prior density in the results. Table 4.2 compares the Dice scores of the segmentations obtained by each method, based on the posterior probabilities (Bayes decision rule) and only on the contextual mixing coefficient maps estimated by each method. Regarding the posterior probabilities case, which is the optimal decision rule, an improvement of about 3 points in Dice with respect to the SVFMM and about 1 point with respect to the $\mathcal{S}$ t-SVFMM was obtained only by changing the prior density. Considering that the prior density acts as a guide during the learning and segmentation process, this improvement in final results is highly valuable given its limited impact. Similarly, results obtained by the NLSVFMM variants only based on the prior probability maps (contextual mixing coefficient maps) outperform the other methods in a similar manner. Obviously, Dice results are significantly lower than those obtained based on the posterior probability (which takes into account also the intensities of the images), however the comparison among them demonstrates the superiority of the NLSVFMM approach.

The evaluation performed on the Berkeley dataset also yielded favorably results in terms of RI for the NLSVFMMs methods. In almost all cases, the NLSVFMMs variants performed superior or comparable to the $\mathcal{S}$ t model, with the advantage that they introduce less parameters that must be estimated. Moreover, as expected, the patchbased NLp-SVFMM method achieved the best results, confirming that the probabilistic NLM weighting function better captures the local redundancy of the images. Only in the $K=3$ case (the simplest segmentation), the SVFMM method outperforms the rest of the models. However, as segmentation complexity increases, the models including edge preserving priors perform better in all cases.

It is worth noting that standard deviations are high and similar for all methods. This is due to several images that are intrinsically difficult to segment and present poor RI results across all methods uniformly, rather than to a high variability in the methods themselves. To corroborate it, we measured the percentage of cases of the Berkeley300 dataset that showed RI improvement when segmented by our methods with respect to the SVFMM and the $\mathcal{S}$ t-SVFMM. For most of the $K$ states, our NLpSVFMM approach showed RI improvement in approximately more than the $85 \%$ of cases compared with the SVFMM, and more than the $80 \%$ of cases compared with the $\mathcal{S}$ t-SVFMM. This behavior indicates that there is a systematic improvement of our algorithms with respect to the previous approaches in the literature, which is not a product of random fluctuations due to the high standard deviations. 
On the other side, it is also worth noting that differences in RI are not significantly large between methods, which is a reasonably behavior. Under the Bayes' decision rule, the prior probability $p(\Pi)$ (or $p(A)$ ) acts as an initial degree of belief of each label at each location of the image before observing the image, which ultimately represents a less informative distribution compared to the class conditional $p(X \mid \Theta, \Pi)$. Therefore, the impact on the final results of changing the prior distributions is limited, and thus the segmentation results are less affected. In addition, prior distributions become weaker as the number of observations in a problem increase, which is the case of pixel classification in an image. In those cases, variations in the prior densities also have a lesser impact in final results, which is observed in our experimentation.

In future work, we plan to study the inclusion of prior distributions for other parameters of the model, i.e. $\mu_{j}$ and $\Sigma_{j}, \forall j$, to introduce prior knowledge and constraints that can help in the estimation of more accurate and realistic models. 
Chapter 4. Non Local Spatially Varying Finite Mixture Models for unsupervised image segmentation 


\section{Chapter 5}

\section{Vascular heterogeneity assessment of glioblastoma through the Hemodynamic Tissue Signature}

Understanding glioblastoma intra- and inter-tumoral heterogeneity represents one of the most important challenges in advancing the fight against this lethal cancer. Over the years, much evidence has accumulated to suggest that this heterogeneity is highly responsible for the poor prognosis of the tumor and its resistance to effective therapies. Specifically, vascular heterogeneity has been identified as one of the most important pathological hallmarks of glioblastoma. The study of the aberrant vasculature of this lethal tumor, its hemodynamic local behavior and its angiogenesis mechanism is crucial to design new effective therapies that improve patient prognosis. However, to date, determining the extent and characteristics of this intra-tumor heterogeneity is still poorly understood.

In this chapter we present the Hemodynamic Tissue Signature (HTS), an unsupervised machine learning method to describe the vascular heterogeneity of glioblastoma by means of perfusion MRI analysis. The method analyzes the perfusion markers to automatically draw four reproducible habitats that describe the tumor vascular heterogeneity: the High Angiogenic Tumor (HAT) and Low Angiogenic Tumor (LAT) habitats of the enhancing tumor, the potentially tumor Infiltrated Peripheral Edema (IPE) and the Vasogenic Peripheral Edema (VPE).

The purpose of the work presented in this chapter was to assess if preoperative vascular heterogeneity of glioblastoma predicts OS of patients undergoing standard of care treatment by using the HTS method. To do so we conducted Kaplan-Meier and Cox proportional hazard analyses to study the prognostic potential of the HTS habitats on a cohort of 50 retrospective patients from a local hospital. Additionally, we explored the ability of the HTS habitats to improve the conventional prognostic models based on clinical, morphological, and demographic features.

The contents of this chapter were published in the journal publications (JuanAlbarracin et al, 2018; Fuster-Garcia et al, 2018)-thesis contributions C3, C4, P5 and $P 6$. 


\subsection{Introduction}

Glioblastoma heterogeneity has been identified as one of the factors responsible for the high aggressiveness of these neoplasms (Lemée et al, 2015) and as a key hallmark to understanding their resistance to effective therapies (Soeda et al, 2015). Molecular characterization of glioblastomas has advanced the understanding of the biology and heterogeneity of these tumors, improving routine diagnosis, prognosis, and response to therapy (Parsons et al, 2008; Verhaak et al, 2010). However, significant interest has been placed in the past years in the analysis of glioblastoma heterogeneity based on medical imaging, to discover non-invasive tumor features related to different clinical outcomes such as OS, tumor grading or molecular sub-typing Wangaryattawanich et al (2015).

Glioblastoma is characterized by highly infiltrative and deeply invasive behavior (Dang et al, 2010). Strong vascular proliferation, robust angiogenesis, and extensive microvasculature heterogeneity are major pathological features that differentiate glioblastomas from low-grade gliomas (Alves et al, 2011; Hardee and Zagzag, 2012; Kargiotis et al, 2006). Such factors have been shown to have a direct effect on prognosis (Hardee and Zagzag, 2012). Therefore, the early assessment of the highly heterogeneous vascular architecture of glioblastomas could provide powerful information to improve therapeutic decision making.

Dynamic Susceptibility Contrast (DSC) MRI has been used widely to retrieve physiologic information on glioblastoma vasculature (Shah et al, 2010; Knopp et al, 1999; Lupo et al, 2005). DSC quantification involves the computation of the hemodynamic indexes obtained from the kinetic analysis of the $\mathrm{T}_{2^{*}}$ concentration time curves retrieved from the first pass of an intravenously injected paramagnetic contrast agent (Østergaard, 2005). Section 2.2.2 performs an in depth description of the techniques employed in this thesis to estimate the perfusion parametric maps from the DSC sequence. Such perfusion indexes have demonstrated powerful capabilities for a wide range of applications such as tumor grading (Law et al, 2003; Emblem et al, 2008), neovascularization assessment (Thompson et al, 2011; Tykocinski et al, 2012), early response to treatment assessment (Vidiri et al, 2012; Elmghirbi et al, 2017), recurrence versus radionecrosis (Hu et al, 2009; Barajas et al, 2009) and prediction of clinical outcome (Mangla et al, 2010; Jain et al, 2014).

Numerous studies have been focused on the analysis of pretreatment perfusion indexes to assess tumor heterogeneity (Jackson et al, 2007; Liu et al, 2017a; SanzRequena et al, 2013; Ulyte et al, 2016). The most common practice is the manual definition of Region Of Interests (ROIs) within the tumor to study vascular properties that correlate with clinical outcomes. However, these manual approaches impair the reproducibility studies and the analysis of high-dimensional multiparametric MRI data (Young et al, 2007).

An alternative novel approach to describe the heterogeneity of glioblastomas is by means of the definition of lesion sub-compartments or radiological habitats that express a specific biological behavior observable from MRI. Several attempts have been made in the literature to describe the glioblastoma heterogeneity through this technique (Dextraze et al, 2017; Zhou et al, 2014; Lee et al, 2015; Zhou et al, 2017). However most of them are based on morphological MRI and classical image processing 
techniques such as histogram analysis, intensity thresholding, texture measurements or histogram derived features. The preoperative characterization of the vascular heterogeneity of glioblastomas through a multiparametric search of habitats drawn from an unsupervised machine learning process has not previously been well established in the literature.

In this sense, we hypothesize that vascular-related habitats obtained in the preoperative evaluation of glioblastoma are early predictors of OS in patients who subsequently undergo standard-of-care treatment. In this work, we present the Hemodynamic Tissue Signature (HTS) method: an unsupervised machine learning-based algorithm that delineates a set of vascular habitats within the glioblastoma obtained through a multiparametric structured clustering of morphologic and DSC MRI features. HTS includes consideration of four habitats: the High Angiogenic Tumor (HAT) (the more perfused area of the enhancing tumor), the Low Angiogenic Tumor (LAT) (the area of the enhancing tumor with a lower angiogenic profile), the potentially Infiltrated Peripheral Edema (IPE) (the surrounding non-enhancing region adjacent to the tumor with elevated perfusion indexes), and the Vasogenic Peripheral Edema (VPE) (the remaining edema with a lower perfusion profile).

To determine whether the preoperative vascular heterogeneity of glioblastoma allows early prediction of OS of patients who undergo standard-of-care treatment, we conducted a survival analysis on the basis of perfusion measures obtained from the HTS habitats. In addition, we also studied the contribution of the HTS habitats to improve the estimation of OS with respect to models based solely on clinical, morphological and demographic variables; and models including perfusion markers measured from the conventional enhancing tumor and edema ROIs instead of HTS habitats.

\subsection{Materials}

\subsubsection{Patient selection}

Our institutional review board approved this retrospective study, and the requirement for patient informed consent was waived. Eighty-four patients from January 2012 to December 2016 with suspected glioblastoma were included. The inclusion criteria were: (a) confirmation of glioblastoma through biopsy; (b) access to preoperative MRI examinations, including unenhanced and GBCA-enhanced $\mathrm{T}_{1}$-weighted, $\mathrm{T}_{2}$-weighted, FLAIR, and DSC sequences; and (c) patients who underwent standard Stupp treatment (Stupp et al, 2005).

Of the 84 initial patients, six were excluded because of an incomplete MRI study, three were excluded because of motion or spike artifacts on the DSC images that prevented the quantification (gamma variate $R^{2}$ goodness of fit $<0.95$ ), 10 patients were excluded because of unconfirmed or unconventional glioblastomas (giant cell glioblastoma and glioblastoma with oligodendroglioma component), and 10 patients were excluded because they did not undergo resection because of their tumor location (only biopsy results available) or they did not undergo radiation therapy and chemotherapy treatment. In addition, five patients who presented with glioblastomas with contiguous leptomeningeal extensions were excluded from the study because of the inability 
Chapter 5. Vascular heterogeneity assessment of glioblastoma through the Hemodynamic Tissue Signature

to accurately differentiate the tumor vascularity from the reactive meningeal enhancement in the perfusion signal intensity. Finally, 50 patients constituted the study group, including 33 men with an average age of 60.94 years (range, 25-80 years); 17 women with an average age of 62.53 years (range, 36-75 years); and an overall mean age of 60.08 years (range, 25-80 years).

\subsubsection{Magnetic Resonance Imaging}

Standard-of-care examinations were obtained with 1.5-T or 3-T imagers (Signa HDxt; GE Healthcare, Waukesha, Wisconsin) with an eight-channel-array head coil. MRI examinations included unenhanced and GBCA-enhanced $\mathrm{T}_{1}$-weighted three-dimensional spoiled gradient-echo sequences with inversion recovery (repetition times msec/echo times msec, 6-10/2-4; matrix, $256 \times 256$; section thickness, $1.5 \mathrm{~mm}$; field of view, $24 \times 24 \mathrm{~cm}$; inversion time, $400 \mathrm{msec}$; flip angle, $\left.70^{\circ}-80^{\circ}\right)$, fast spin-echo $\mathrm{T}_{2}$-weighted imaging (3000-4000/100-110; matrix, 256 ×256; section thickness, $5 \mathrm{~mm}$; field of view, $21.9 \times 21.9 \mathrm{~cm}$; one signal acquired; intersection gap, $2 \mathrm{~mm}$ ) and a FLAIR sequence (8000-9000/140-165; matrix, $256 \times 192$; section thickness, $5 \mathrm{~mm}$; field of view, $22 \times 22$ $\mathrm{cm}$; one signal acquired; intersection gap, $2 \mathrm{~mm}$; inversion time, $2200 \mathrm{msec}$ ).

Table 5.1: Summary of the most relevant parameters of the MR studies employed in the study.

\begin{tabular}{lccccc}
\hline & $\mathrm{T}_{1}$ & $\mathrm{~T}_{1 C E}$ & $\mathrm{~T}_{2}$ & FLAIR & DSC \\
MR techinque & $\begin{array}{c}\text { Spoiled } \\
\text { gradient-echo }\end{array}$ & $\begin{array}{c}\text { Spoiled } \\
\text { gradient-echo }\end{array}$ & $\begin{array}{c}\text { Fast } \\
\text { spin-echo }\end{array}$ & $\begin{array}{c}\text { Fluid Attenuated } \\
\text { Inversion Recovery }\end{array}$ & Gradient-echo \\
TE (msec) & $6-10$ & $6-10$ & $3000-4000$ & $8000-9000$ & 25 \\
TR (msec) & $2-4$ & $2-4$ & $100-110$ & $140-165$ & 2000 \\
Matrix (mm) & $256 \times 256$ & $256 \times 256$ & $256 \times 256$ & $256 \times 192$ & $128 \times 128$ \\
Sect. thickness (mm) & 1.5 & 1.5 & 5 & 5 & 7 \\
Field of view (cm) & $24 \times 24$ & $24 \times 24$ & $21.9 \times 21.9$ & $22 \times 22$ & $14 \times 14$ \\
Flip angle & $70^{\circ}-80^{\circ}$ & $70^{\circ}-80^{\circ}$ & $90^{\circ}$ & $90^{\circ}$ & $60^{\circ}$ \\
Inv. time (msec) & 400 & 400 & & 2200 & 0.1 \\
Bolus-conct. (mmol/kg) & & & & & \\
Bolus-speed (mL/sec) & & & & & 5 \\
Number of dynamics & & & & & \\
\hline
\end{tabular}

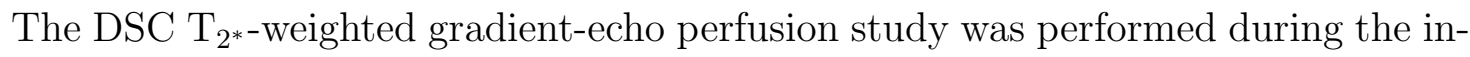
jection of GBCA (Multihance; Bracco, Milan, Italy). A bolus injection of $0.1 \mathrm{mmol} / \mathrm{kg}$ of GBCA was administered at $5 \mathrm{~mL} / \mathrm{sec}$ by using a power injector (no pre-bolus administration). Saline solution was injected after the bolus injection. The study was performed with the following parameters: 2000/25; matrix, $128 \times 128(1.8 \times 1.8 \mathrm{~mm}$ in-plane resolution); section thickness, $7 \mathrm{~mm}$; flip angle, $60^{\circ}$; $14 \mathrm{~cm}$ full-coverage craniocaudal (20 sections), 40 sequential temporally equidistant volumes, each one with an acquisition time of 2 seconds. The baseline before injection of the bolus was five dynamics. Table 5.1 summarizes the aforementioned parameters. 


\subsection{Methods}

\subsubsection{Quantification of DSC parametric maps}

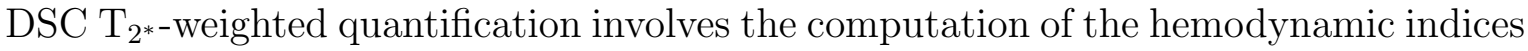
obtained from a kinetic analysis of the first pass of a intravenously injected paramagnetic contrast agent (Østergaard, 2005). A detailed explanation of the calculation of perfusion parametric maps is performed in section 2.2.2. In order for this chapter to be self-contained, a short remainder will be made.

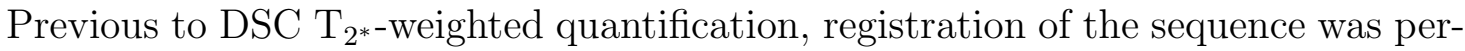
formed to the morphologic $\mathrm{T}_{2}$-weighted image of the patient. Next, signal intensity curves were converted to concentration time curves by using the following equation 2.1. Contrast material leakage correction was performed by using the technique proposed by Boxerman et al (2006) and recirculation was corrected by means of gamma-variate curve fitting (please refer to section 2.2.2 for more details).

rCBV and $\mathrm{rCBF}$ maps were obtained using standard algorithms previously described (Knutsson et al, 2010). rCBV was computed according to numerical integration of the area under the curve of the gamma-variate fittings (Knutsson et al, 2010); while rCBF was calculated by means of the block-circulant SVD deconvolution technique proposed by $\mathrm{Wu}$ et al (2003). Both perfusion maps were normalized against the contra-lateral unaffected white matter value.

The AIF was automatically selected by following a divide-and-conquer approach. The method recursively dichotomizes the set of concentration time curves of the perfusion study into two groups, selecting those curves with higher peak height, earliest time to peak, and lowest full-width at half maximum. We used the median as a threshold to split the groups. The process is repeated until 10 or fewer curves are conserved, finally fixing the AIF as the average of those curves.

\subsubsection{Enhancing tumor and edema segmentation}

In this work the enhancing tumor and edema ROI delineation was performed by using an unsupervised segmentation method based on a variant of the work proposed in a study by Juan-Albarracín et al (2015b). This method is based on DCM-SVFMM (Nikou et al, 2007), which consists of a clustering algorithm that combines GMM with continuous DCAGMRFs to take advantage of the self-similarity and local redundancy of the images. The method includes the unenhanced and GBCA-enhanced $\mathrm{T}_{1}$-weighted sequences, the $\mathrm{T}_{2}$-weighted sequence, and the FLAIR sequence in combination with atlas-based prior knowledge of healthy tissues to perform the segmentation. The automated enhancing tumor and edema ROIs obtained with the method were manually revised and validated by two experienced radiologists in consensus (F.A., with 14 years of experience; L.M.B., with 25 years of experience).

Nowadays, this method has been replaced by a more robust and powerful approach based on CNNs (Juan-Albarracín et al, 2019a) (to be presented in the next chapter), which has proven to be comparable and competitive with manual segmentations performed by expert radiologists. The new method achieves a Dice score index of 0.89 on a large public real glioblastomas dataset, of more than 300 cases manually annotated 
Chapter 5. Vascular heterogeneity assessment of glioblastoma through the Hemodynamic Tissue Signature

by more than 3 expert radiologist each case.

\subsubsection{Hemodynamic Tissue Signature habitats}

The HTS consists of a set of vascular habitats detected in glioblastomas and obtained by means of a multiparametric unsupervised analysis of DSC MRI patterns within the tumor. The technology used to compute the HTS of the glioblastoma is publicly accessible for non-commercial research purposes at https://www.oncohabitats.upv.es.

The HTS defines four habitats within the glioblastoma: the High Angiogenic Tumor (HAT) and Low Angiogenic Tumor (LAT) habitats, and the Infiltrated Peripheral Edema (IPE) and Vasogenic Peripheral Edema (VPE) habitats. Table 5.2 summarizes the relationships among HTS habitats, glioblastoma tissue, and DSC observed vascularity.

Table 5.2: Glioblastoma habitats considered by the HTS an their relation with the degree of vascularity and the pathological tissue.

\begin{tabular}{lll}
\hline HTS habitat & Vascularity & Glioblastoma-related tissue \\
HAT & Highest & High-angiogenic enhancing tumor \\
LAT & High & Low-angiogenic enhancing tumor \\
IPE & Low & Potentially tumor infiltrated peripheral edema \\
VPE & Lowest & Vasogenic peripheral edema \\
\hline
\end{tabular}

HTS habitats are obtained by means of a DCM-SVFMM structured clustering (with the DCAGMRF prior) of $\mathrm{rCBV}$ and $\mathrm{rCBF}$ maps. The clustering consists of two stages (see figure 5.1): (a) a two-class clustering of the $\mathrm{rCBV}$ and $\mathrm{rCBF}$ data at the whole enhancing tumor and edema ROIs defined morphologically; and (b) a two-class clustering also of the $\mathrm{rCBV}$ and $\mathrm{rCBF}$ data within each ROI obtained in stage a). To ensure the reproducibility of the HTS, both stages are initialized with a deterministic seed method. We fix the seeds of every two-class clustering to the extremes of the rCBV and rCBV distributions (5\% and $95 \%$ percentiles, respectively, for each class).

The aim of the first stage is to refine the enhancing tumor and the edema ROIs, previously delineated through the anatomical MRI segmentation, but introducing the perfusion information. Therefore, a spatially varying mixture of two components is fit to the distributions of $\mathrm{rCBV}$ and $\mathrm{rCBF}$ observed in the regions previously labeled as enhancing tumor and edema. We named these two components: enhancing tumor at DSC $\left(E T_{D S C}\right)$ and edema at DSC $\left(E D_{D S C}\right)$. During the fitting process, we introduce several constraints to avoid misclassifications of nearby healthy vascular structures $E T_{D S C}$. Thus, we constrained the apparition of the $E T_{D S C}$ class to a neighborhood of less than $1 \mathrm{~cm}$ around the enhancing tumor observed on the GBCA-enhanced $\mathrm{T}_{1^{-}}$ weighted MRI (Guo et al, 2016). This constraint allows the correction of misalignments during the DSC registration and the removal of healthy vascular structures far from the enhancing area of the tumor, which may distort the HTS. Moreover, the also enforce 


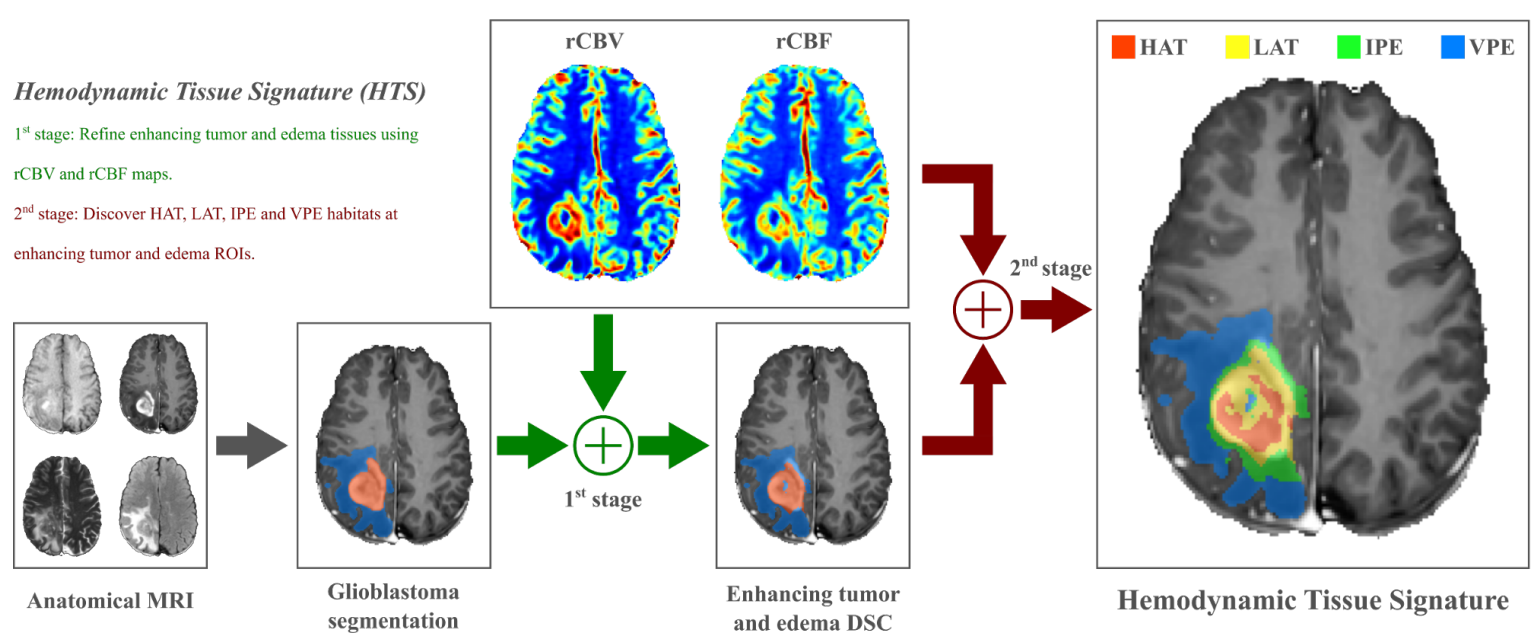

Figure 5.1: Schema of the methodology used to compute the HTS of the glioblastoma. HAT = high-angiogenic tumor; LAT = low-angiogenic tumor, IPE = inflitrated peripheral edema, $\mathrm{VPE}=$ vasogenic peripheral edema

the $E T_{D S C}$ class to explain at least the $80 \%$ of the enhancing tumor ROI obtained from the anatomical segmentation.

The second stage includes DCM-SVFMM clustering within the $E T_{D S C}$ and $E D_{D S C}$ ROIs, to delineate the two potential hemodynamic habitats inside each tissue. Likewise, the first stage, a spatially varying mixture of two components is fit to the distributions of rCBV and rCBF for both the $E T_{D S C}$ and $E D_{D S C}$ ROIs. In this stage, several constraints are also introduced. First, we force a minimum size of the habitats of at least $10 \%$ of the whole lesion ROI to avoid habitat vanishing. Second, infiltrated peripheral edema habitat is also constrained to a nearby region around the $E T_{D S C}$ class. Following the definition of the clinical target volume proposed by Guo et al (2016), we fix a $2 \mathrm{~cm}$ margin around the enhancing tumor observed at GBCAenhanced $\mathrm{T}_{1}$-weighted MRI as the maximum distance where the infiltrated peripheral edema habitat could appear. Voxels classified as infiltrated peripheral edema outside this region are automatically removed from the HTS, since they have a similar vascular pattern to that of infiltrated peripheral edema but far from the plausible region of tumor infiltration.

\subsubsection{Statistical analysis}

All analyses were performed with software (Matlab R2015a; MathWorks, Natick, Massachusetts) on a personal workstation.

First, an evaluation of the statistical differences between habitats was conducted to assess the degree of separability of the rCBV and $\mathrm{rCBF}$ distributions within each habitat to confirm their different hemodynamic activity. To do so, global probabilistic deviation (Sáez et al, 2017) was used as a metric to control the degree of concordance among several statistical distributions. Such a metric is bounded to a $[0,1]$ range, with 0 referring to absolute overlapping between distributions, and 1 indicating non-overlapped, completely separated distributions. Therefore, for each perfusion parameter, the distributions of the four proposed habitats and their global probabilistic 
deviation metric were calculated.

Second, Cox proportional hazards modeling was conducted to investigate the relationship between patient survival and the $r C B V_{\max }$ and $r C B F_{\max }$ at the HTS habitats. We used the maximum of the perfusion parameters because it has been reported to be the most reliable measure for inter-observer and intra-observer reproducibility (Wetzel et al, 2002). Proportional hazard ratios with $95 \%$ confidence intervals were reported, while the Wald test was used to determine the significance of the Cox regression model results.

Finally, Kaplan-Meier survival analyses between populations dichotomized according to the median value of each perfusion biomarker at each HTS habitat were also conducted. A log-rank test was used to determine the statistical significance of the differences in observed population survival. Average survival for each population was also reported. Benjamini-Hochberg false discovery rate correction at an $\alpha$ level of .05 was used to correct for multiple hypothesis testing (Benjamini and Hochberg, 1995) in all analyses.

On the other side, we also conducted a study to determine the added value of HTS habitats for predicting patient OS when these are added to classical models based on clinical, demographic and morphological variables. To do so, first we conducted an analysis to measure the importance of each clinical, demographic or MRI-related variable independently to predict OS. For the categorical variables we conducted Kaplan-Meier survival analysis, while for the continuous variables Cox proportional hazards modeling was used.

Next, we studied the added value of the HTS habitats by adding its information to the models built with the variables that showed significant association with OS in the aforementioned independent study. To do so we constructed three models:

Model 1: Clinical variables + demographic variables

Model 2: Clinical variables + demographic variables $+r C B V_{\max }$ and $r C B F_{\max }$ at enhancing tumor

Model 3: Clinical variables + demographic variables $+r C B V_{\max }$ and $r C B F_{\max }$ at HTS habitats

Cox proportional hazard regression models were fit for each model and a comparison between the predicted OS and the real patient's OS was performed. A Kaplan-Meier survival study was next performed by using Cox the predicted OS of each model to split the population in two groups: long-survivors, whose Cox predicted OS was greater than the real average OS of the population (402 days); and short-survivors, whose Cox predicted survival was less or equal than the real average OS of the population (402 days). Root Mean Squared Error (RMSE) was employed to measure the deviance between the predicted OS and the real OS of the population. Single-tailed Wilcoxon paired signed rank test was used to determine if there are stastistically significant differences between predicted RMSEs among models. 


\subsection{Results}

Figure 5.2 shows examples of HTS maps. GBCA-enhanced $\mathrm{T}_{1}$-weighted and $\mathrm{T}_{2^{-}}$ weighted images, as well as $\mathrm{rCBV}$ and $\mathrm{rCBF}$ maps are shown with the HTS map of the patient. The global probabilistic deviation analysis of the different hemodynamic activity among habitats yielded the following average results: $0.88 \pm 0.03$ for $\mathrm{rCBV}$ and $0.86 \pm 0.05$ for $\mathrm{rCBF}$. These results indicate separated perfusion distributions between habitats of the patients. Figure 5.3 shows an example of the rCBV distributions for each HTS habitat. The Cox proportional hazard analysis is presented in Table 5.3.

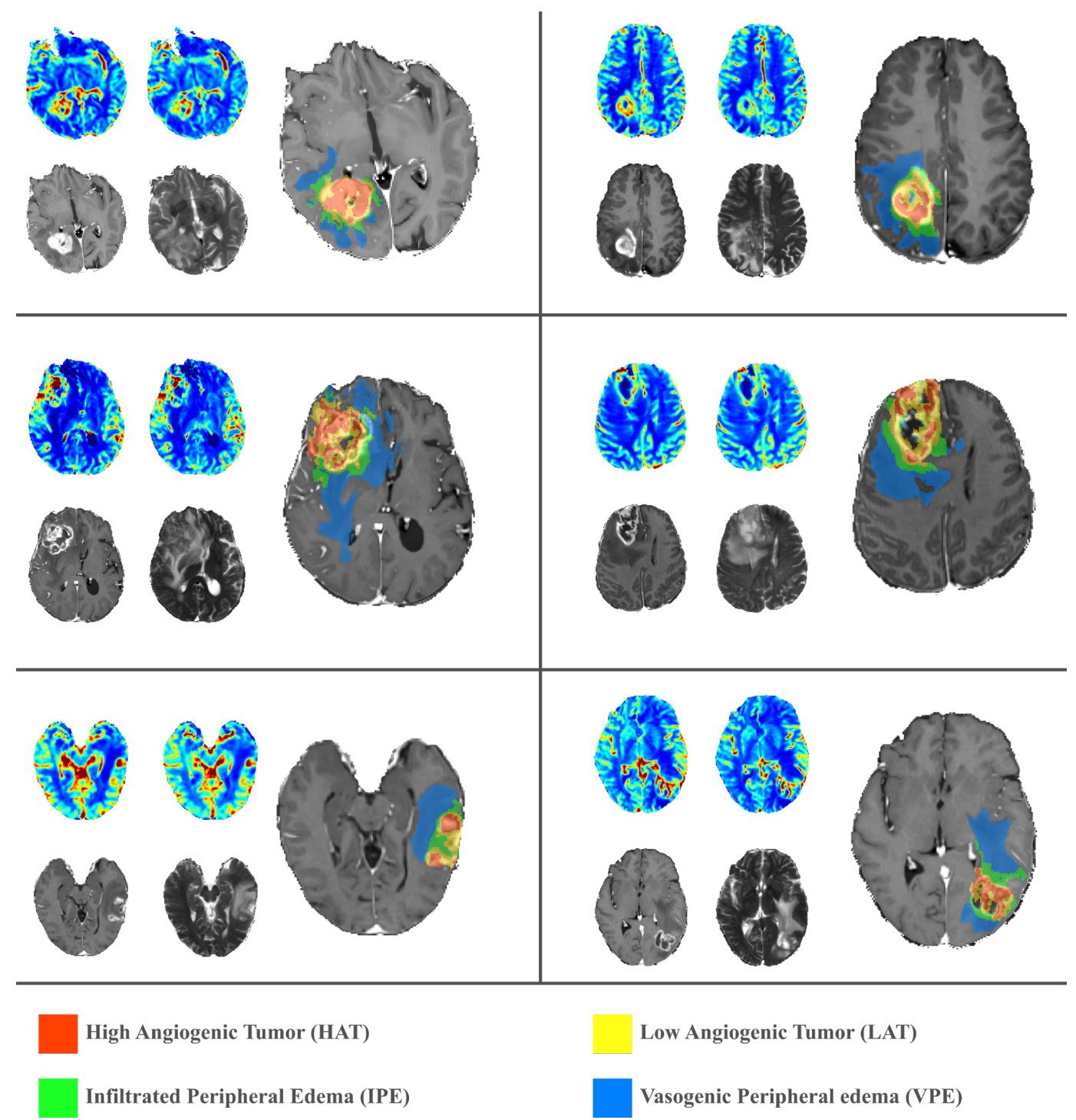

Figure 5.2: Examples of the HTS habitat's map placed over the GBCA-enhanced $\mathrm{T}_{1}$-weighted MR image for six patients. In addition, for each patient, GBCA-enhanced $\mathrm{T}_{1}$-weighted $\mathrm{MR}$ image, $\mathrm{T}_{2^{-}}$ weighted MR image, rCBV map, rCBF map, are also shown in small images (left to right, top to bottom).

Significant results were obtained for $r C B V_{\max }$ and $r C B F_{\max }$ in the high-angiogenic habitat (hazard ratios, $1.22[P=.0004]$ and $1.20[P=.0004]$, respectively), $r C B V_{\max }$ 
Chapter 5. Vascular heterogeneity assessment of glioblastoma through the Hemodynamic Tissue Signature

Table 5.3: Cox proportional hazard analysis for maximum perfusion indexes at the HTS habitats to predict patient's OS.

\begin{tabular}{|c|c|c|}
\hline Habitat and perfusion parameter & Hazard ratio & P-value ${ }^{*}$ \\
\hline \multicolumn{3}{|l|}{ High-angiogenic tumor } \\
\hline$r C B V_{\max }$ & $1.22(1.10,1.35)$ & $.0004^{\dagger}$ \\
\hline$r C B F_{\max }$ & $1.20(1.09,1.32)$ & $.0004^{\dagger}$ \\
\hline \multicolumn{3}{|l|}{ Low-angiogenic tumor } \\
\hline$r C B V_{\max }$ & $1.62(1.31,2.01)$ & $.0001^{\dagger}$ \\
\hline$r C B F_{\max }$ & $1.89(1.35,2.66)$ & $.0005^{\dagger}$ \\
\hline \multicolumn{3}{|l|}{ Infiltrated peripheral edema } \\
\hline$r C B V_{\max }$ & $1.67(1.05,2.66)$ & $.0498^{\dagger}$ \\
\hline$r C B F_{\max }$ & $2.07(1.02,4.20)$ & .0579 \\
\hline
\end{tabular}

Vasogenic peripheral edema

\begin{tabular}{lll}
$r C B V_{\max }$ & $1.59(0.94,2.70)$ & .0962 \\
$r C B F_{\max }$ & $1.58(0.71,3.54)$ & .2657 \\
\hline
\end{tabular}

Data in parentheses are $95 \%$ confidence intervals.

* False discovery rate corrected.

$\dagger$ Indicates a significant difference.

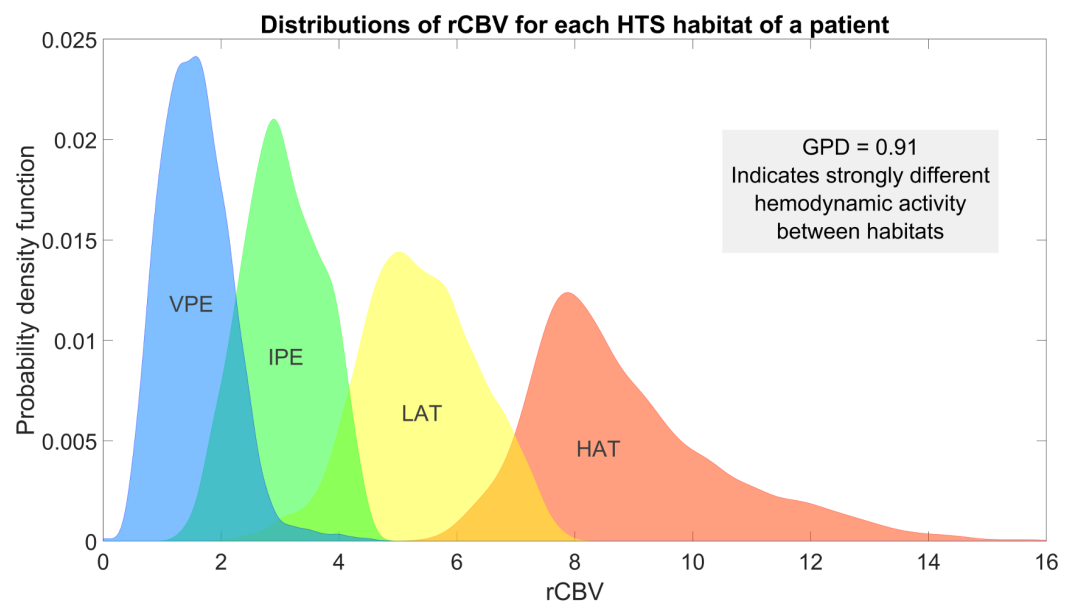

Figure 5.3: Example of the rCBV distributions at each HTS habitat for a 62-year-old man and the global probabilistic deviation score obtained in the assessment of the separability of the distributions. HAT $=$ high-angiogenic tumor, LAT $=$ low-angiogenic tumor, IPE $=$ infiltrated peripheral edema, $\mathrm{VPE}=$ vasogenic peripheral edema.

and $r C B F_{\max }$ in the low-angiogenic habitat (hazard ratios, $1.62[P=.0001]$ and 1.89 $[P=.0005]$, respectively) and $r C B V_{\max }$ in the infiltrated peripheral edema habitat 
(hazard ratio, $1.67[P=.498])$. Non-significant results were obtained for $r C B F_{\max }$ in the infiltrated peripheral edema (hazard ratio, $2.07[P=.0579]$ ), and $r C B V_{\max }$ in the vasogenic peripheral edema habitat (hazard ratio, $1.59[P=.0962])$ and $r C B F_{\max }$ at vasogenic peripheral edema (hazard ratio, $1.58[P=.2657]$ ). Figure 5.4 shows the scatterplots of the combinations of perfusion biomarkers and HTS habitats that yielded significant correlation in the Cox survival analysis. Total versus partial maximum safe resection, complete versus incomplete concomitant radiation therapy and chemotherapy and adjuvant temozolomide plus bevacizumab administration are also shown.

Kaplan-Meier survival analysis yielded significant differences for the survival times observed for the populations dichotomized by low and high $r C B V_{\max }$ in the highangiogenic habitat (log-rank test $P=.0104$ ), $r C B F_{\max }$ in the high-angiogenic habitat (log-rank test $P=.0003$ ), $r C B V_{\max }$ at low-angiogenic habitat (log-rank test $P=$ .0048 ) and $r C B F_{\max }$ in the low-angiogenic habitat (log-rank test $P=.0128$ ). An average difference of 230 days in overall survival between populations was observed. Mean survival of the population was 459 days \pm 286.15 (range, 121-1656 days). Table 5.4 shows the average observed survival times for each population and the corrected P-values for the log-rank survival test. Figure 5.5 demonstrates the Kaplan-Meier estimated survival functions for the different populations dichotomized according to the $r C B V_{\max }$ and $r C B F_{\max }$ at different HTS habitats.

Table 5.4: Kaplan-Meier survival analysis for populations with high versus low maximum perfusion parameters at the different habitats.

\begin{tabular}{|c|c|c|c|}
\hline \multirow[b]{2}{*}{ Habitat and perfusion parameter } & \multicolumn{2}{|c|}{ Average survival (days) } & \multirow[b]{2}{*}{ P-value* } \\
\hline & Low & High & \\
\hline \multicolumn{4}{|l|}{ High-angiogenic tumor } \\
\hline$r C B V_{\max }$ & 550.33 & 351.78 & $.0104^{\dagger}$ \\
\hline$r C B F_{\max }$ & 594.73 & 311.96 & $.0003^{\dagger}$ \\
\hline \multicolumn{4}{|l|}{ Low-angiogenic tumor } \\
\hline$r C B V_{\max }$ & 571.62 & 337 & $.0048^{\dagger}$ \\
\hline$r C B F_{\max }$ & 554.96 & 355.04 & $.0128^{\dagger}$ \\
\hline \multicolumn{4}{|l|}{ Infiltrated peripheral edema } \\
\hline$r C B V_{\max }$ & 500.52 & 423.63 & .7986 \\
\hline$r C B F_{\max }$ & 557.17 & 368.38 & .0641 \\
\hline \multicolumn{4}{|l|}{ Vasogenic peripheral edema } \\
\hline$r C B V_{\max }$ & 532.56 & 385.44 & .1300 \\
\hline$r C B F_{\max }$ & 500.92 & 420.31 & .8992 \\
\hline
\end{tabular}

* False discovery rate corrected.

$\dagger$ Indicates a significant difference. 
Chapter 5. Vascular heterogeneity assessment of glioblastoma through the Hemodynamic Tissue Signature
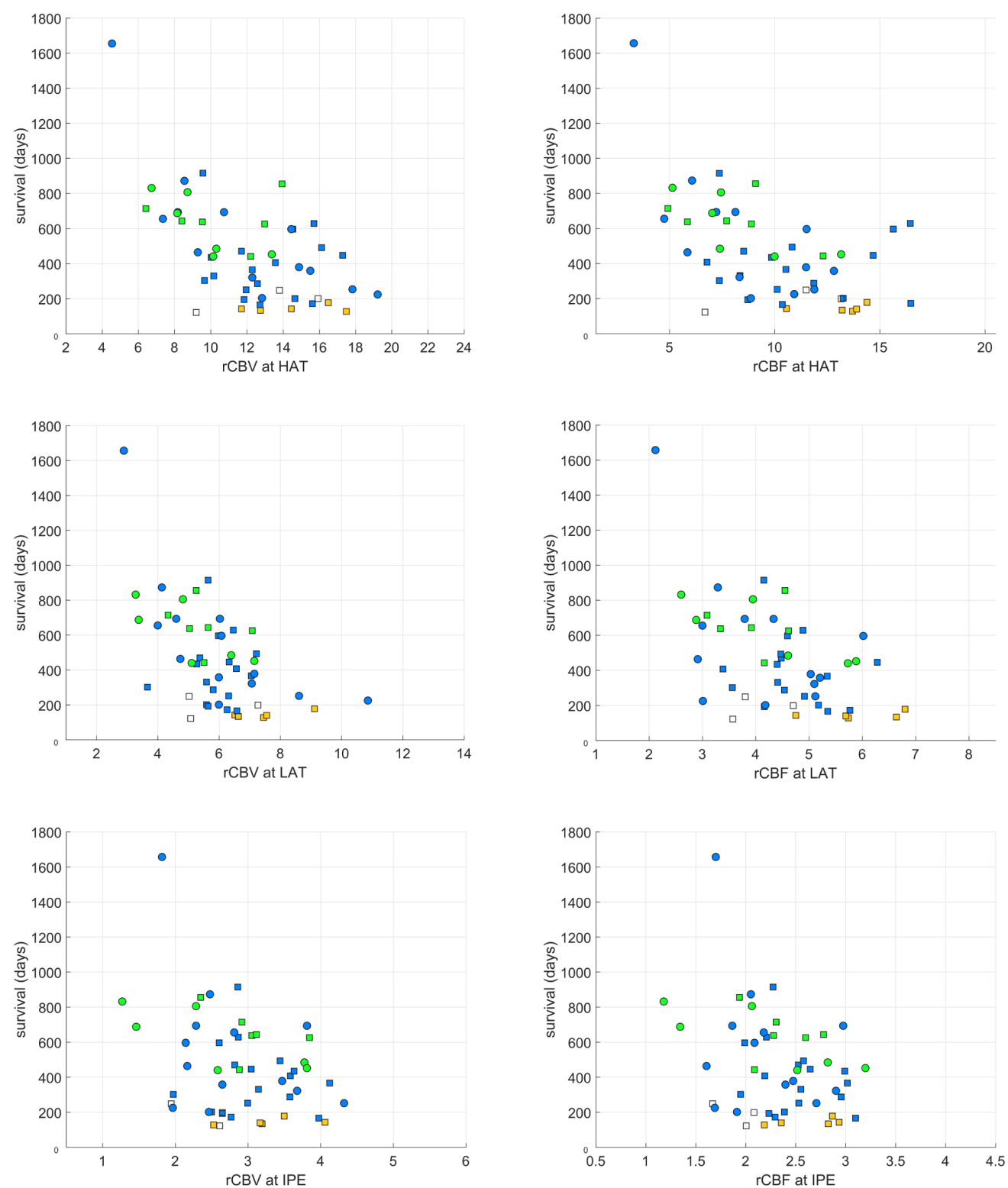

\begin{tabular}{|lll|}
\hline Color: & Shape: & Color filled: \\
Adjuvant TMZ and BVZ & $\bigcirc$ Total resection & $\bigcirc \square$ Complete concomitant RT-QT \\
Adjuvant TMZ but not BVZ & $\square$ Partial resection & $\bigcirc \square$ Incomplete concomitant RT-QT \\
Neither adjuvant TMZ nor BVZ & & \\
\hline
\end{tabular}

Figure 5.4: Scatterplots of the relation between patient survival and perfusion biomarkers in High Angiogenic Tumor (HAT), Low Angiogenic Tumor (LAT) and Infiltrated Peripheral Edema (IPE) habitats. Treatment undergone by each patient is also shown, to allow differentiation between total versus partial resection, complete versus incomplete concomitant radiation therapy and chemotherapy, adjuvant temozolomide and bevacizumab administration. $\mathrm{BVZ}=$ bevacizumab, RT-QT $=$ radiation therapy-chemotherapy, TMZ = temozolomide.

Tables 5.5 and 5.6 show the log-rank test and the Cox-Wald test to measure the potential of each categorical and additional continuous variable to independently correlate with patient OS. 

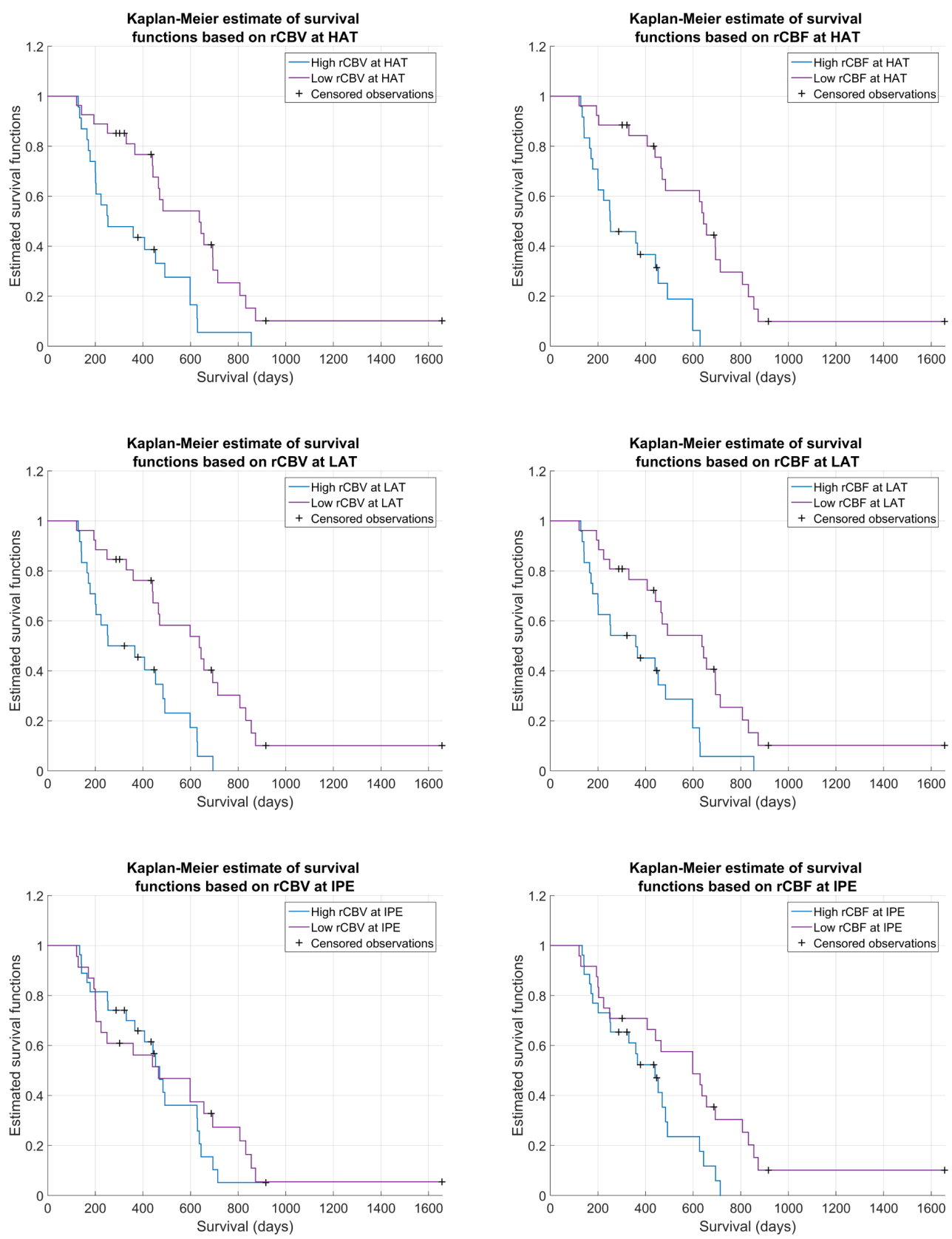

Figure 5.5: Kaplan-Meier estimated survival functions for the populations divided by the median $r C B V_{\max }$ or $r C B F_{\max }$ in High Angiogenic Tumor (HAT), Low Angiogenic Tumor (LAT) and Infiltrated Peripheral Edema (IPE) habitats.

Figure 5.6 shows the OS predictions generated by Models 1, 2 and 3 during the leave-one-out evaluation. Model 1, based solely on clinical and demographic variables, obtained a $R M S E=219.33$; Model 2, based on clinical, demographic and perfusion markers at enhancing tumor region, obtained a $R M S E=202.39$; while Model 3, which was based on clinical, demographic and perfusion markers measured from the HTS habitats, obtained a $R M S E=183.57$.

The improvement achieved in the prognostic estimation obtained by the Model 2 with respect the Model 1 was $7.7 \%$, while the improvement obtained by the Model 
Chapter 5. Vascular heterogeneity assessment of glioblastoma through the Hemodynamic Tissue Signature

Table 5.5: Kaplan-Meier survival analysis for clinical and demographic variables included in the study.

\begin{tabular}{llll}
\hline Variable & \# Population & Average survival & P-value $^{*}$ \\
Gender & {$[36,24]$} & {$[437,349]$} & 0.522 \\
Laterality & {$[24,33]$} & {$[487,371]$} & 0.525 \\
Resection & & & \\
$\quad$ Total & {$[20,40]$} & {$[562,322]$} & $.025^{\dagger}$ \\
$\quad$ Subtotal & {$[34,26]$} & {$[356,462]$} & .262 \\
Biopsy & {$[6,54]$} & {$[129,432]$} & $.001^{\dagger}$ \\
Distance to ventricles & & & \\
$\quad$ Long & {$[20,40]$} & {$[575,316]$} & $.002^{\dagger}$ \\
Mid & {$[21,39]$} & {$[391,408]$} & .957 \\
Short & {$[19,41]$} & {$[233,481]$} & $3 \mathrm{e}-5^{\dagger}$ \\
Location & & & \\
Frontal & {$[14,46]$} & {$[299,433]$} & $.028^{\dagger}$ \\
Parietal & {$[14,46]$} & {$[465,383]$} & .217 \\
Temporal & {$[27,33]$} & {$[391,411]$} & .962 \\
Occipital & {$[5,55]$} & {$[573,386]$} & .525 \\
Radiochemotherapy & & & $.006^{\dagger}$ \\
Complete & {$[49,11]$} & {$[465,122]$} & $3 \mathrm{e}-11^{\dagger}$ \\
Incomplete & {$[5,55]$} & {$[173,423]$} & \\
\hline
\end{tabular}

Note that this study was carried out with 10 patients more than the previous studies.

* False discovery rate corrected.

$\dagger$ Indicates a significant difference.

3, which incorporates the HTS habitats, reached $16.3 \%$ in terms of RMSE. Wilcoxon paired signed test yielded no significant differences between Model 1 and Model 2, however it did obtained statistically significant differences between Model 3 and Model $1(P<.05)$.

\subsection{Discussion}

In this study, we investigated whether the perfusion heterogeneity in the four vascular habitats of the HTS is predictive of survival in untreated glioblastomas. Our 
Table 5.6: Cox proportional hazard analysis for continuous variables included in the study.

\begin{tabular}{|c|c|c|}
\hline Variable & Hazard ratio & P-value* \\
\hline Age & $1.08(1.01,1.07)$ & $.007^{\dagger}$ \\
\hline \multicolumn{3}{|c|}{ Enhancing tumor } \\
\hline$r C B V_{\max }$ & $1.23(1.10,1.37)$ & $.0004^{\dagger}$ \\
\hline$r C B F_{\max }$ & $1.24(1.11,1.37)$ & $8 \mathrm{e}-5^{\dagger}$ \\
\hline Volumetry & $1.02(1.00,1.03)$ & $.012^{\dagger}$ \\
\hline \multicolumn{3}{|l|}{ Edema } \\
\hline$r C B V_{\max }$ & $1.20(0.94,1.54)$ & .134 \\
\hline$r C B F_{\max }$ & $1.28(0.93,1.75)$ & .127 \\
\hline Volumetry & $1.00(0.99,1.01)$ & .979 \\
\hline \multicolumn{3}{|l|}{ HAT } \\
\hline$r C B V_{\max }$ & $1.14(1.06,1.23)$ & $6 e-4^{\dagger}$ \\
\hline$r C B F_{\max }$ & $1.16(1.08,1.39)$ & $4 e-5^{\dagger}$ \\
\hline Volumetry & $1.06(1.01,1.11)$ & $.011^{\dagger}$ \\
\hline \multicolumn{3}{|l|}{ LAT } \\
\hline$r C B V_{\max }$ & $1.28(1.07,1.52)$ & $.007^{\dagger}$ \\
\hline$r C B F_{\max }$ & $1.44(1.07,1.93)$ & $.015^{\dagger}$ \\
\hline Volumetry & $1.03(1.01,1.06)$ & $.006^{\dagger}$ \\
\hline \multicolumn{3}{|l|}{ IPE } \\
\hline$r C B V_{\max }$ & $1.89(1.07,3.34)$ & $.027^{\dagger}$ \\
\hline$r C B F_{\max }$ & $2.57(1.12,5.91)$ & $.027^{\dagger}$ \\
\hline Volumetry & $1.01(0.98,1.05)$ & .401 \\
\hline \multicolumn{3}{|l|}{ VPE } \\
\hline$r C B V_{\max }$ & $1.84(0.99,3.42)$ & .052 \\
\hline$r C B F_{\max }$ & $2.31(0.95,5.64)$ & .065 \\
\hline Volumetry & $1.00(0.99,1.01)$ & .917 \\
\hline
\end{tabular}

Note that this study was carried out with 10 patients more than the previous studies.

Data in parentheses are $95 \%$ confidence intervals.

* False discovery rate corrected.

$\dagger$ Indicates a significant difference. 

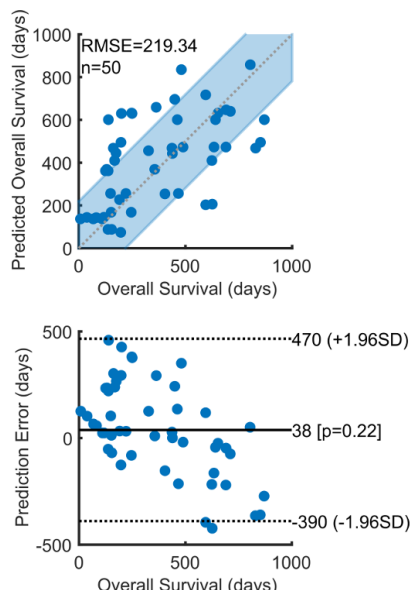
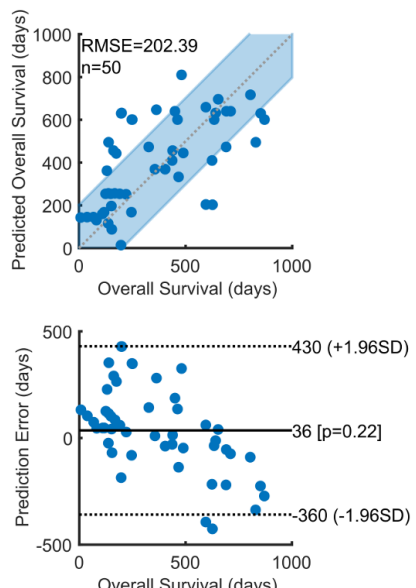
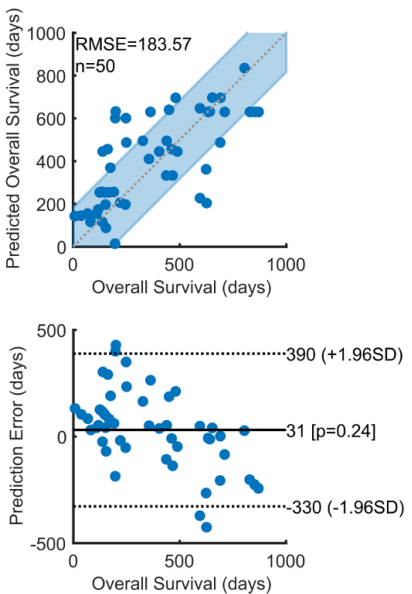

Figure 5.6: Scatter plot for predicted versus real OS (top) and Bland-Altman plot (bottom) for the proposed aforementioned models: Model 1 (left), Model 2 (middle), and Model 3 (right). Red dashed line indicates the hypothetical perfect prediction, while shadow blue bands indicate the confidence intervals of the prediction.

results demonstrate that the preoperative perfusion heterogeneity contains relevant information about patient survival, even considering the effect of other known relevant factors such as standard-of-care treatment. Gradually longer survival times were found for patients who presented with lower preoperative perfusion indexes in different HTS habitats. The influence of standard-of-care treatment on patient survival was also directly observed. As expected, patients who underwent maximal safe resection plus concomitant adjuvant chemotherapy and radiation therapy showed better survival times. However, a tendency of longer survival times within subgroups of patients who underwent the same specific treatment and had lower perfusion indexes at several habitats was also observed. This indicates that preoperative perfusion heterogeneity contains early important information about patient survival.

Cox proportional hazard analysis substantiated these conclusions. High- and lowangiogenic habitats arose as those with the highest prognostic abilities, yielding significant correlations between survival and $r C B V_{\max }$ and $r C B F_{\max }$ (with multiple-test false discovery rate correction). $r C B V_{\max }$ in the infiltrated peripheral edema habitat also was significantly correlated with survival, while $r C B F_{\max }$ in the infiltrated peripheral edema was significantly correlated without multiple-test correction $(P=.0434)$. These results suggest that relevant information about patient survival is also contained in the peripheral edema (Jain et al, 2014; Akbari et al, 2014; Artzi et al, 2014).

Significant differences also were observed in the Kaplan-Meier estimated survival functions for populations divided according to the median $r C B V_{\max }$ and $r C B F_{\max }$ at several HTS habitats. An improvement of approximately 230 days in overall survival was observed for patients who had lower $r C B V_{\max }$ and $r C B F_{\max }$ in the high- and lowangiogenic habitats. These results support the potential of HTS to accurately describe the preoperative vascular heterogeneity of glioblastomas and its prognostic abilities at early stages.

Regarding the added value provided by the HTS to predict OS in combination with clinical and demographic variables, we found that, in agreement with the literature, 
variables such as age, biopsy instead of resection, long or short distance of the lesion to the ventricles, frontal tumor location, complete vs incomplete radiochemotherapy or enhancing tumor volumetry presented strong association with patient OS.

The comparison of the prognostic capabilities of the three proposed regression models also confirmed the added value of the HTS markers in the prognosis estimation of the patient. As expected, the models including perfusion MRI performed better than those based solely on clinical and demographic variables. However, it is worth noting that the inclusion of perfusion related measurements from the enhancing tumor ROI improved the prognosis estimation by $7.7 \%$ in terms of RMSE, however, the improvement obtained when including the HTS perfusion-based measurements outperformed the previous model by $16.3 \%$. Additionally, only this latter improvement proved to be statistically significant. These results reinforce the evidence of the need for a better characterization of the heterogeneity of glioblastoma in order to improve the current management of the disease.

Several studies have been conducted to analyze the vascular heterogeneity of the glioblastoma, many of them focusing on the enhancing tumor region. Law et al (2008) found that patients who presented with an $r C B V_{\max }$ of less than 1.75 in the enhancing tumor had longer progression-free survival times; however, they did not find a significant correlation with overall survival. Sawlani et al (2010) also correlated time to progression with several hyper-perfused regions delineated in the $r C B V$. However, they also observed no significant correlation between patients' overall survival and $r C B V_{\max }$ in the enhancing tumor. Hirai et al (2008) and Jain et al (2013), studied the potential for prediction of survival of $r C B V$ in the enhancing tumor of high-grade gliomas. They showed that patients who presented with an $r C B V_{\max }$ of greater than 2.3 had significantly shorter survival times. These results are consistent with our findings, hence aligning HTS with the results of previous studies in the literature. However, these studies were only based on the $r C B V_{\text {max }}$; other perfusion indexes such as $r C B F_{\text {max }}$, which may also add important information about instantaneous capillary flow in the tissues to the analysis, were not considered. Moreover, manual delineation of ROIs based on $r C B V_{\max }$ was used, which may affect reproducibility and may not fully capture the tumor information available in a multiparametric study.

Authors of other studies focused on peripheral edema of glioblastoma. Akbari et al (2014), Jain et al (2014) and Artzi et al (2014) studied the peritumoral region of the glioblastoma to account for heterogeneity and possible tumor infiltration in the peripheral edema. Akbari et al used ROIs to train a support vector machine, which was then used to generate heterogeneity maps. Jain et al analyzed the association of Visually Accessible Rembrandt Images, or VASARI, features and molecular data with overall survival and progression-free survival, while Artzi et al used diffusion, perfusion, and morphologic MR imaging with an unsupervised segmentation algorithm to analyze the edema region. Their results correlate with our findings in the infiltrated peripheral edema habitat, because they also found that vascular heterogeneity in the peripheral edema correlate with overall patient survival. However, ROIs to describe tumor heterogeneity in these studies were also delineated manually. Moreover, statistical tests were conducted without multiple comparison correction, which decreases the statistical power of the conclusions. 
One of the main limitations of our study and similar studies in which authors attempted to describe the vascular heterogeneity of glioblastoma by means of discovery of new habitats was the unavailability of a ground truth for validating the habitat's segmentation (Akbari et al, 2014; Hirai et al, 2008; Jain et al, 2013). Multiple biopsy or pathological sampling could confirm the accuracy of the habitats; however, such techniques cannot always be performed in clinical practice. To overcome this limitation, alternative validation should be conducted to demonstrate the clinical relevance of the habitats. In this study, we have analyzed the relationship between the preoperative vascular heterogeneity of glioblastomas described through HTS and patient survival.

Another important limitation was the lack of molecular markers in the population of our study. Molecular markers are currently considered a standard of care for WHO glioblastoma classification and are also known to affect prognosis of patients with glioblastoma. Positive correlation of genetic markers with the HTS habitats would strengthen the study and the predictive potential of the proposed method and should be performed in the future.

Finally, a limitation of the HTS arises in the presence of highly vascularized healthy structures close to the glioblastoma, such as nearby vessels or arteries. In such cases, these structures can be misidentified as high- and low-angiogenic or infiltrated peripheral edema habitats depending on their degree of vascularity. Although HTS implements several constraints to remove these healthy structures, nearby vessels may influence the HTS, modifying measures obtained from the habitats. Results of future studies should improve vessel detection by using a vascular probability atlas to weight the HTS inference process.

In conclusion, preoperative vascular heterogeneity of glioblastomas demonstrated by the habitats of HTS is associated with patient survival. HTS separates glioblastomas into four vascular habitats with early prognostic capabilities, offering an opportunity to define refined imaging biomarkers surrogated to clinical outcomes. 


\section{Chapter 6}

\section{Multi-center international validation of the Hemodynamic Tissue Signature for glioblastoma}

In the previous chapter it was shown that early-stage vascular heterogeneity of glioblastoma has a direct effect on prognosis and a strong association with tumor aggressiveness. The proposed Hemodynamic Tissue Signature (HTS) method provides an unsupervised ML solution to study the vascular heterogeneity of glioblastomas by analyzing patterns of local hemodynamic activity in perfusion MRI. The four habitats delineated by the HTS method have demonstrated strong associations with patient OS and early prognostic capabilities. However, the validation of the method was conducted on a single-center cohort of patients, thus avoiding the variability inherent in real-life multi-center heterogeneous scenarios.

In this chapter we present a multi-center retrospective international validation of the HTS method. The validation was performed under the umbrella of the clinical study NCT03439332, which involved seven international centers with more than 180 patients. The purpose of this chapter is to validate the association between the hemodynamic markers obtained from the HTS habitats and the patient OS, considering the inter-center variability of MRI acquisition protocols, patient demographics and lesion heterogeneity. Kaplan-Meier and Cox proportional hazard analyses were conducted to study the prognostic potential of the HTS habitats under the proposed environment.

The contents of this chapter were published in the journal publications (JuanAlbarracin et al, 2018; Álvarez Torres et al, 2019) -thesis contributions C4, C5, P5, $P 6$ and $P$ r .

\subsection{Introduction}

Glioblastoma is the most aggressive malignant primary brain tumor in adults with a median survival rate of 12-15 months (Louis et al, 2016; Gately et al, 2017). It still carries a poor prognosis despite aggressive treatment, which includes tumor resection followed by chemo-radiotherapy (Bae et al, 2018; Akbari et al, 2014). One of the main factors thought to be responsible of glioblastoma aggressiveness is its vascular 
heterogeneity (Akbari et al, 2014; Soeda et al, 2015), mainly defined by a strong angiogenesis that supplies the glioblastoma metabolic requirements and accounts for its rapid progression (Weis and Cheresh, 2011; De Palma et al, 2017). The early-vascular profile of the tumor is strongly associated with molecular characteristics of the lesion (De Palma et al, 2017), which in combination with the local micro-environment are both directly related to the glioblastoma progression (Weis and Cheresh, 2011).

The negative association between patient survival rates and vascular markers extracted from perfusion MRI has been widely demonstrated in the literature (Akbari et al, 2014; Jain et al, 2014; Jensen et al, 2014). Perfusion indexes such as rCBV or capillary heterogeneity were found to be associated with prognosis and patient survival rates. Dozens of methodologies are proposed in the literature to assess these perfusion indexes, ranging from manually defined ROIs, which introduce high uncertainty and lack of repeatability; to more up-to-date techniques based on artificial intelligence methods able to analyze imaging patterns to describe tumor heterogeneity (Demerath et al, 2017; Jena et al, 2016; Price et al, 2016; Chang et al, 2017; Cui et al, 2016).

In 2018, Juan-Albarracín et al (2018) proposed the Hemodynamic Tissue Signature (HTS) method able to characterize the vascular heterogeneity of glioblastomas by means of delineating vascular habitats obtained from perfusion MRI. The HTS method draws four habitats within the lesion related to: the High Angiogenic Tumor (HAT) region, the Low Angiogenic Tumor (LAT) region, the potentially Infiltrated Peripheral Edema (IPE) and the Vasogenic Peripheral Edema (VPE) habitats. The HTS method is publicly accessible at ONCOhabitats website https://www.oncohabitats.upv.es for non-commercial research purposes.

The study conducted by Juan-Albarracín et al (2018) found statistically significant correlations between OS and several measures obtained from the HTS markers. In 2018, Fuster-Garcia et al (2018) demonstrated the ability of these imaging markers to improve the prognosis of conventional models based on clinical, morphological and demographic features. Both studies were conducted on a single-center cohort of 50 patients from a local institution.

However, the current road map to validate an imaging marker into clinical routine requires to overcome two translational gaps (Abramson et al, 2015; O'Connor et al, 2017): the marker validation with pre-clinical or clinical datasets from a single or a few expert centers, and the subsequent extension of the evaluation to multiple centers, along with the biological validation of the biomarkers. The aforementioned studies of Juan-Albarracín et al (2018) and Fuster-Garcia et al (2018) addressed the first translational gap, however it is still necessary to validate the HTS markers in a multicenter heterogeneous cohort with the purpose of demonstrating their robustness and stability under highly variable clinical conditions.

The purpose of this work is to determine if the habitats obtained by the HTS method are predictive of the OS of glioblastoma patients undergoing standard-of-care treatment. To this end, we have involved the HTS technology in an international multicenter observational retrospective clinical study registered at the ClinicalTrial.gov official platform with name "Multicentre Validation of How Vascular Biomarkers From Tumor Can Predict the Survival of the Patient With Glioblastoma (ONCOhabitats)" and identifier NCT03439332. We have analyzed the possible association between the 
HTS markers and patients OS, as well as their capability to stratify groups of patients according to these markers, in a large heterogeneous international cohort. Additionally, we also have assessed the robustness of the HTS method operating under a highly variable MRI acquisition protocols from multiple centers.

\subsection{Materials}

\subsubsection{Patient selection}

Seven European clinical centers participated in the clinical study NCT03439332: the Hospital Universitario de La Ribera, Alzira, Spain; Hospital de Manises, Manises, Spain; Hospital Clinic, Barcelona, Spain; Hospital Universitario Vall d'Hebron, Barcelona, Spain; Azienda Ospedaliero-Universitaria di Parma, Parma, Italy; Centre Hospitalier Universitaire de Liege, Liege, Belgium and the Oslo University Hospital, Oslo, Norway.

A material transfer agreement document was approved by all the participating centers and an acceptance report was issued by the ethical committee of each center. The institution review board of each center also approved this retrospective study and the requirement for patient-informed consent was waived.

The inclusion criteria for patients participating in the study were: (a) adult patients (age $>18$ y.o.) with histopathological confirmation of glioblastoma diagnosed between January 1, 2012 and January 1, 2018; (b) access to preoperative MRI studies, including: pre- and post-gadolinium $\mathrm{T}_{1}$-weighted, $\mathrm{T}_{2}$-weighted, FLAIR and DSC $\mathrm{T}_{2^{*} \text {-weighted }}$ perfusion sequences; and (c) patients who underwent standard Stupp treatment (Stupp et al, 2005) with a minimum survival of 30 days.

From the initial cohort consisting of 196 patients, two cases were excluded due to incomplete DSC perfusion acquisitions; five cases were excluded due to excessive noise in DSC concentration curves that prevented quantification (gamma variate goodness of fit $R^{2}<0.95$ ); four cases were excluded due to MRI processing errors; and one case was excluded due to inability to differentiate between tumor vascularity and reactive meningeal enhancement. Table 6.1 summarizes the number of patients initially contributed by each center and the number of patients finally excluded due to noncompliance with the inclusion criteria.

Table 6.1: Number of patients contributed by each center with their corresponding excluded due to noncompliance of the inclusion criteria.

\begin{tabular}{lcccccccc}
\hline & H. Ribera & H. Manises & C. Barcelona & H. Vall d'Hebron & AO Parma & CH Liege & Oslo UH & Total \\
Initial & 10 & 14 & 28 & 34 & 42 & 34 & 34 & 196 \\
Excluded & 3 & 0 & 3 & 1 & 2 & 1 & 2 & 15 \\
Enrolled & 7 & 14 & 25 & 33 & 40 & 33 & 32 & 184 \\
\hline
\end{tabular}

The final cohort enrolled in the study was of 184 patients. Those who were still alive during the study were considered as censored observations. The date of censorship was the last date of contact with the patient or, if was not available, the date of the last MRI exam. Table 6.2 summarizes the most important demographic and clinical characteristics of the population. 
Chapter 6. Multi-center international validation of the Hemodynamic Tissue Signature for glioblastoma

Table 6.2: Summary of demographic and clinical variables of the cohort of 184 patients enrolled in the study.

\begin{tabular}{|c|c|c|c|c|c|c|c|c|}
\hline & H. Ribera & H. Manises & C. Barcelona & H. Vall d'Hebron & AO Parma & CH Liege & Oslo UH & Total \\
\hline \multicolumn{9}{|l|}{ Gender $(\mathrm{F} / \mathrm{M})$} \\
\hline \# of patients & $6 / 1$ & $5 / 9$ & $10 / 15$ & $14 / 19$ & $12 / 28$ & $11 / 22$ & $8 / 24$ & $66 / 118$ \\
\hline \multicolumn{9}{|c|}{ Age at diagnosis (years) } \\
\hline Mean & 49 & 65 & 56 & 60 & 61 & 58 & 63 & 60 \\
\hline Range & {$[24,67]$} & {$[39,79]$} & {$[35,74]$} & {$[30,81]$} & {$[35,76]$} & {$[32,77]$} & {$[40,81]$} & {$[24,81]$} \\
\hline \multicolumn{9}{|l|}{ Survival (months) } \\
\hline Mean & 14.6 & 14.4 & 10.3 & 15.2 & 11.7 & 15.3 & 15.4 & 13.7 \\
\hline Median & 9.1 & 12.8 & 9.6 & 13.0 & 12.9 & 14.5 & 12.6 & 12.6 \\
\hline Range & {$[3.4,52.6]$} & {$[3.4,38.4]$} & {$[1.3,26.9]$} & {$[4.1,40.0]$} & {$[1.1,30.7]$} & {$[2.5,41.0]$} & {$[3.0,36.9]$} & {$[1.1,52.6]$} \\
\hline \multicolumn{9}{|c|}{ Resection (\# of patients) } \\
\hline Total & 3 & 3 & 0 & 12 & 19 & 22 & 11 & 70 \\
\hline Sub-total & 1 & 4 & 1 & 10 & 15 & 6 & 21 & 67 \\
\hline Biopsy & 1 & 7 & 6 & 11 & 2 & 5 & 0 & 32 \\
\hline Unknown & 2 & 0 & 9 & 0 & 4 & 0 & 0 & 15 \\
\hline \multicolumn{9}{|c|}{ Tumor location (\# of patients) } \\
\hline Frontal & 2 & 4 & 7 & 10 & 18 & 11 & 12 & 64 \\
\hline Parietal & 2 & 0 & 5 & 7 & 4 & 9 & 3 & 30 \\
\hline Temporal & 3 & 7 & 11 & 13 & 12 & 9 & 14 & 69 \\
\hline Occipital & 0 & 2 & 1 & 2 & 2 & 0 & 1 & 8 \\
\hline Other/Unknown & 0 & 1 & 1 & 1 & 4 & 4 & 2 & 13 \\
\hline \multicolumn{9}{|l|}{ IDH1 (\# of patients) } \\
\hline Mutated & 2 & 0 & 4 & 0 & 0 & 0 & 1 & 6 \\
\hline Wild type & 2 & 0 & 4 & 32 & 30 & 34 & 31 & 99 \\
\hline Unknown & 3 & 14 & 17 & 1 & 10 & 0 & 1 & 79 \\
\hline
\end{tabular}

\subsubsection{Magnetic Resonance Imaging}

Standard-of-care MR examinations were obtained with 1.5-T or 3-T imagers. Pre- and post-GBCA $\mathrm{T}_{1}$-weighted MRI, as well as $\mathrm{T}_{2}$-weighted, FLAIR and DSC perfusion MRI sequences were collected from each center. Table 6.3 summarizes the MRI acquisition protocol employed by each center.

\subsection{Methods}

\subsubsection{Vascular heterogeneity assessment of glioblastoma based on HTS habitats}

The HTS method, available at ONCOhabitats (https://www.oncohabitats.upv.es), was used to describe the vascular heterogeneity of the glioblastomas enrolled in the multicenter study. The methodology comprises the following stages:

1. MRI preprocessing: including denoising, magnetic field inhomogeneity correction, multi-modal registration, brain extraction, motion correction and intensity standardization. 
Table 6.3: Summary of the most relevant parameters of the MRI studies collected by each center. (MFS: Magnetic Field Strength)

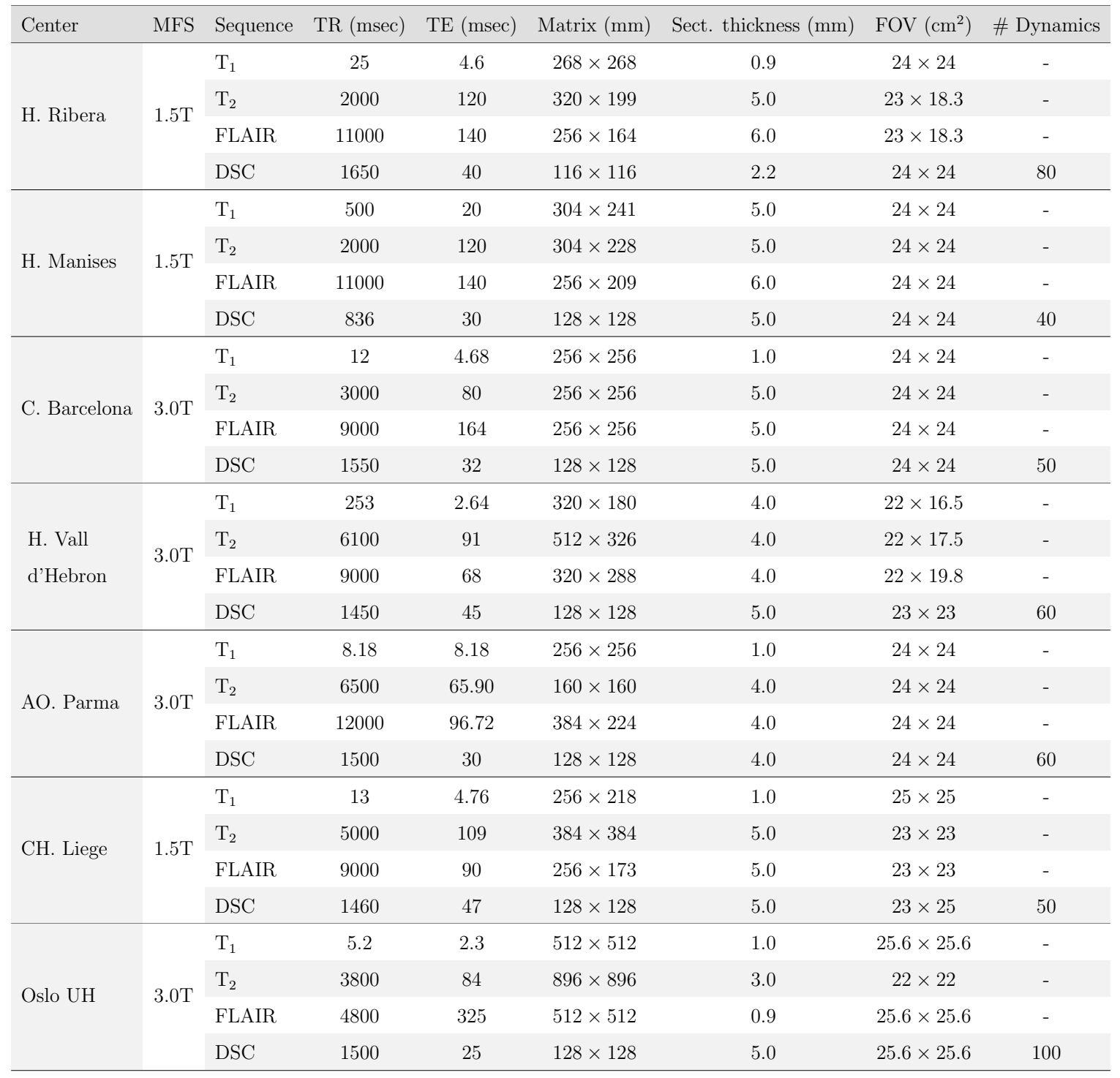

2. Glioblastoma segmentation: implementing a state-of-the-art deep learning 3D CNN that delineates the enhancing tumor, edema and necrotic tissues.

3. Perfusion quantification: to calculate the parametric $\mathrm{rCBV}, \mathrm{rCBF}, \mathrm{MTT}$ and K2 maps derived from the DSC perfusion sequence.

4. HTS habitats: in which an unsupervised segmentation algorithm performs the detection of the HAT, LAT, IPE and VPE habitats to describe the vascular heterogeneity within the lesion.

According to Wetzel et al (2002), for each habitat we defined the HTS marker as the maximum rCBV $\left(r C B V_{\max }\right)$, computed as the $95^{\text {th }}$ percentile of the rCBV distribution within the region defined by the corresponding habitat. 


\subsubsection{Association among OS and HTS markers - whole cohort study}

Cox proportional hazard regression analysis was used to quantify the associations between patient OS and HTS markers. The proportional Hazard Ratios (HRs) with their 95\% confidence intervals were reported, as well as the associated P-values corrected for multiple-test with Benjamini-Hochberg false discovery rate correction at an $\alpha$ level of .05.

Kaplan-Meier analyses were also conducted to study the survival evolution of the population stratified into two groups according to HTS markers: the high-vascular and the low-vascular groups. We defined the high-vascular and low-vascular groups as the set of patients with a $r C B V_{\max }$ higher or lower than an optimal cut-off threshold calculated with the C-index method. Log-rank test was used to determine the statistical differences between the estimated survival functions of the aforementioned groups.

\subsubsection{Association among OS and HTS markers - inter-center study}

In order to determine the degree of agreement in describing the vascular heterogeneity of glioblastomas, we conducted an study measuring the similarities of the HTS marker distributions among the clinical centers enrolled in the study. To this end we conducted a pair-wise Mann-Whitney U-test $(\alpha=.05)$, followed by a post-hoc Tukey's honest significant difference criterion test.

Cox regression analyses were also conducted to assess whether the association between patient OS and HTS markers differed among the centers. Kaplan-Meier analyses were conducted after dividing the population of each center using the same cut-off thresholds previously calculated for the whole cohort study.

All the statistical analyses were performed on Matlab R2017b (MathWorks, Natick, MA).

\subsection{Results}

\subsubsection{Association among OS and HTS markers - whole cohort study}

Table 6.4 summarizes the Cox proportional hazard analysis between HTS markers and patient OS. Statistically significant negative associations were found for $r C B V_{\max }$ at HAT, LAT and IPE habitats and patient OS, with the IPE marker showing the highest HR (1.28). Kaplan-Meier results are presented in Table 6.5, including estimated optimal cut-off thresholds, the number of patients assigned to each group, the estimated C-index area under the curve, the median OS calculated per group, and the log-rank P-values.

Significant differences in OS between low and high vascular groups divided by HTS marker values were found. Consistently with previous results in the literature (Juan- 
Albarracín et al, 2018), patients with a low $r C B V_{\max }$ at HAT, LAT and IPE habitats presented a higher median survival rate.

Table 6.4: Cox regression analysis for $r C B V_{\max }$ at each vascular habitat and patient OS.

\begin{tabular}{ccccc}
\hline & Hazard Ratio & $\begin{array}{c}95 \% \text { confidence } \\
\text { interval }\end{array}$ & P-value & $\begin{array}{c}\text { FDR-corrected } \\
\text { P-value }\end{array}$ \\
$r C B V_{\text {max }}$ & & & & \\
HAT & 1.05 & {$[1.01,1.09]$} & $0.0115^{\dagger}$ & $0.0174^{\dagger}$ \\
LAT & 1.11 & {$[1.02,1.20]$} & $0.0131^{\dagger}$ & $0.0174^{\dagger}$ \\
IPE & 1.28 & {$[1.05,1.55]$} & $0.0122^{\dagger}$ & $0.0174^{\dagger}$ \\
VPE & 1.19 & {$[0.89,1.60]$} & $0.2502^{\dagger}$ & 0.2502 \\
\hline
\end{tabular}

$\dagger$ Indicates a significant difference.

Table 6.5: Kaplan Meier and log-rank test results for patient stratification in low- and high-vascular groups according to $r C B V_{\max }$ value at the HTS habitats.

\begin{tabular}{cccccc}
\hline & $\begin{array}{c}\text { Cut-off } \\
\text { threshold }\end{array}$ & $\begin{array}{c}\text { \# Patients per group } \\
{[\text { low, high }]}\end{array}$ & $\begin{array}{c}\text { AUC } \\
\text { C-Index }\end{array}$ & $\begin{array}{c}\text { OS per group } \\
{[\text { low, high }]}\end{array}$ & P-value \\
$r C B V_{\text {max }}$ & & & & & \\
HAT & 11.06 & {$[97,87]$} & 0.606 & {$[14.3,11.3]$} & $0.0014^{\dagger}$ \\
LAT & 5.31 & {$[91,93]$} & 0.605 & {$[13.9,11.3]$} & $0.0085^{\dagger}$ \\
IPE & 1.92 & {$[59,125]$} & 0.634 & {$[14.3,11.4]$} & $0.0101^{\dagger}$ \\
VPE & 1.67 & {$[100,84]$} & 0.599 & {$[13.8,11.2]$} & 0.1356 \\
\hline
\end{tabular}

AUC: Area under the curve.

$\dagger$ Indicates a significant difference.

Figure 6.1 shows the Kaplan-Meier estimated survival functions for the populations divided in high-vascular and low-vascular groups according to the optimal-cutoff threshold for the $r C B V_{\max }$ estimated with the C-Index method.

\subsubsection{Association among OS and HTS markers - inter-center study}

No statistical differences were found between the $r C B V_{\max }$ values at the different HTS habitats among most of the centers, specially for the IPE habitat, which was the most correlated with OS in the whole cohort study of section 6.4.2 (see tables 6.6, 6.7, 6.8, $6.9 ; \dagger$ indicates statistical significant difference). 
Chapter 6. Multi-center international validation of the Hemodynamic Tissue Signature for glioblastoma
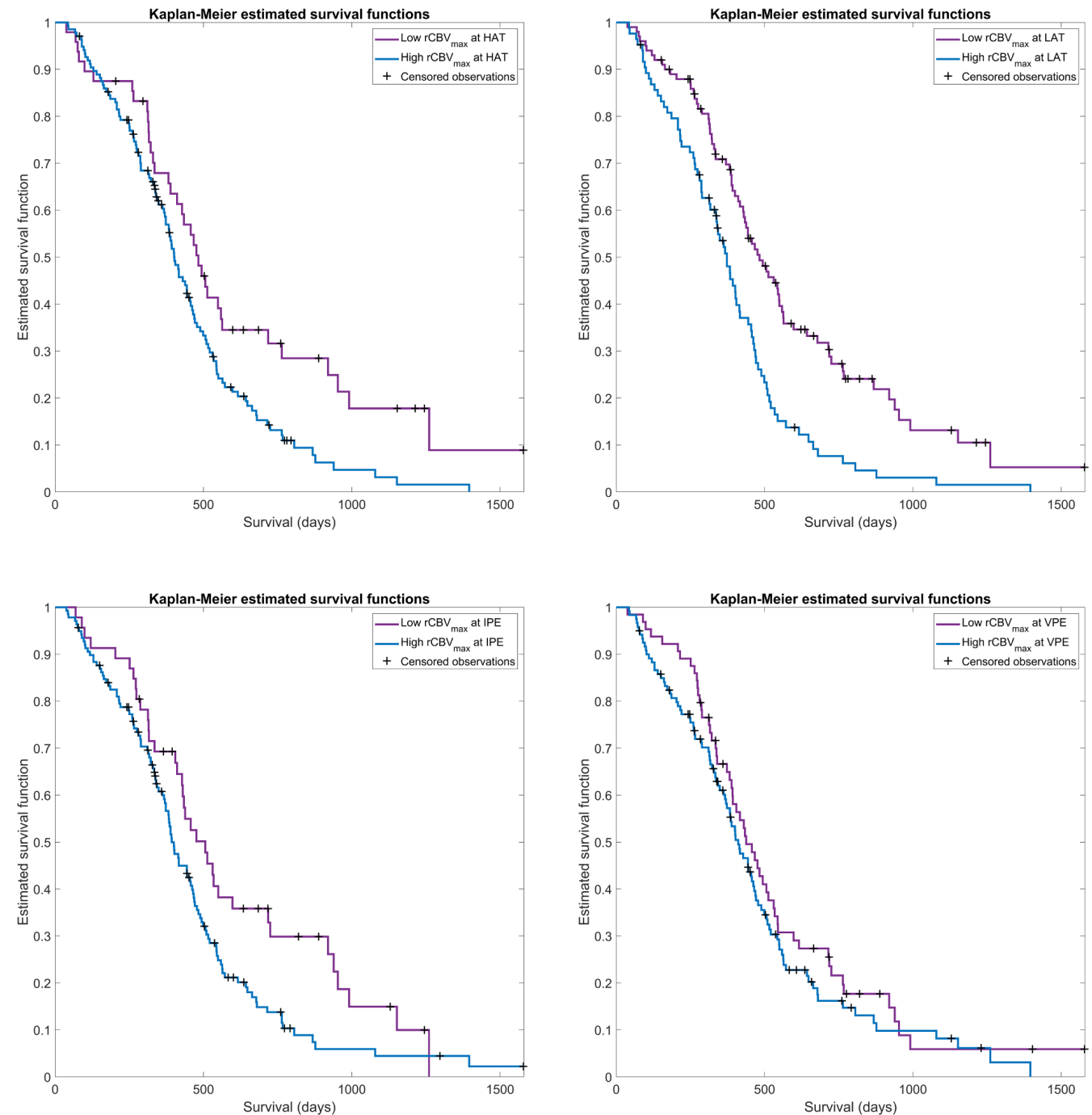

Figure 6.1: Kaplan-Meier estimated survival functions for the populations stratified into groups according to high and low $r C B V_{\max }$ at HAT (top left), LAT (top right), IPE (bottom left) and VPE (bottom right) HTS habitats. 
Table 6.6: Pair-wise Mann Whitney U-test results for $r C B V_{\max }$ at HAT habitat.

\begin{tabular}{lcccccc}
\hline & H. Manises & C. Barcelona & H. Vall d'Hebron & AO Parma & CH Liege & Oslo UH \\
H. Ribera & 0.3139 & 0.6816 & 0.1762 & 0.1937 & 0.4765 & 0.0959 \\
H. Manises & & $0.0363^{\dagger}$ & 0.6170 & 0.7899 & 0.1111 & 0.7292 \\
C. Barcelona & & & $0.0258^{\dagger}$ & $0.0124^{\dagger}$ & 0.2091 & $0.0017^{\dagger}$ \\
H. Vall d'Hebron & & & & 0.6063 & 0.1302 & 0.1584 \\
AO Parma & & & & & 0.0504 & 0.3557 \\
CH Liege & & & & & & 0.0085 \\
\hline
\end{tabular}

Table 6.7: Pair-wise Mann Whitney U-test results for $r C B V_{\max }$ at LAT habitat.

\begin{tabular}{l|cccccc}
\hline & H. Manises & C. Barcelona & H. Vall d'Hebron & AO Parma & CH Liege & Oslo UH \\
\hline H. Ribera & 0.0932 & 0.3384 & 0.1445 & 0.0914 & 0.2401 & $0.0135^{\dagger}$ \\
H. Manises & & 0.1243 & 0.6170 & 0.7003 & 0.2794 & 0.5586 \\
C. Barcelona & & & 0.1317 & 0.932 & 0.5300 & $0.0103^{\dagger}$ \\
H. Vall d'Hebron & & & & 0.7690 & 0.3832 & 0.0844 \\
AO Parma & & & & & 0.2423 & 0.1392 \\
CH Liege & & & & & & 0.0228 \\
\hline
\end{tabular}

Table 6.8: Pair-wise Mann Whitney U-test results for $r C B V_{\max }$ at IPE habitat.

\begin{tabular}{lcccccc}
\hline & H. Manises & C. Barcelona & H. Vall d'Hebron & AO Parma & CH Liege & Oslo UH \\
\cline { 2 - 4 } & 0.0932 & 0.0917 & 0.1016 & 0.2500 & 0.1349 & 0.1383 \\
H. Ribera & & 0.8262 & 0.6170 & 0.2910 & 0.7011 & 0.3457 \\
H. Manises & & & 0.8752 & 0.3771 & 0.9624 & 0.5253 \\
C. Barcelona & & & & 0.2949 & 0.9387 & 0.6227 \\
H. Vall d'Hebron & & & & & 0.2848 & 0.7296 \\
AO Parma & & & & & & 0.6698 \\
CH Liege & & & & & & \\
\hline
\end{tabular}

Table 6.9: Pair-wise Mann Whitney U-test results for $r C B V_{\max }$ at VPE habitat.

\begin{tabular}{l|cccccc}
\hline & H. Manises & C. Barcelona & H. Vall d'Hebron & AO Parma & CH Liege & Oslo UH \\
\cline { 2 - 7 } & 0.0676 & 0.0754 & $0.0299^{\dagger}$ & 0.1937 & $0.0462^{\dagger}$ & 0.4103 \\
H. Ribera & & 1.0000 & 0.8615 & 0.2030 & 0.8433 & 0.0678 \\
H. Manises & & & 0.8260 & 0.2275 & 0.9374 & 0.0546 \\
C. Barcelona & & & & 0.0931 & 0.8174 & $0.0160^{\dagger}$ \\
H. Vall d'Hebron & & & & & 0.1117 & 0.5595 \\
AO Parma & & & & & & $0.0280^{\dagger}$ \\
CH Liege & & & & & & \\
\hline
\end{tabular}


Box-whisker plot shown in Figure 6.2 summarizes the information contained in tables $6.6,6.7,6.8,6.9$. Significant overlapping among the distributions of $r C B V_{\max }$ of each hospital can be observed, indicating no statistical differences in the HTS markers among centers.

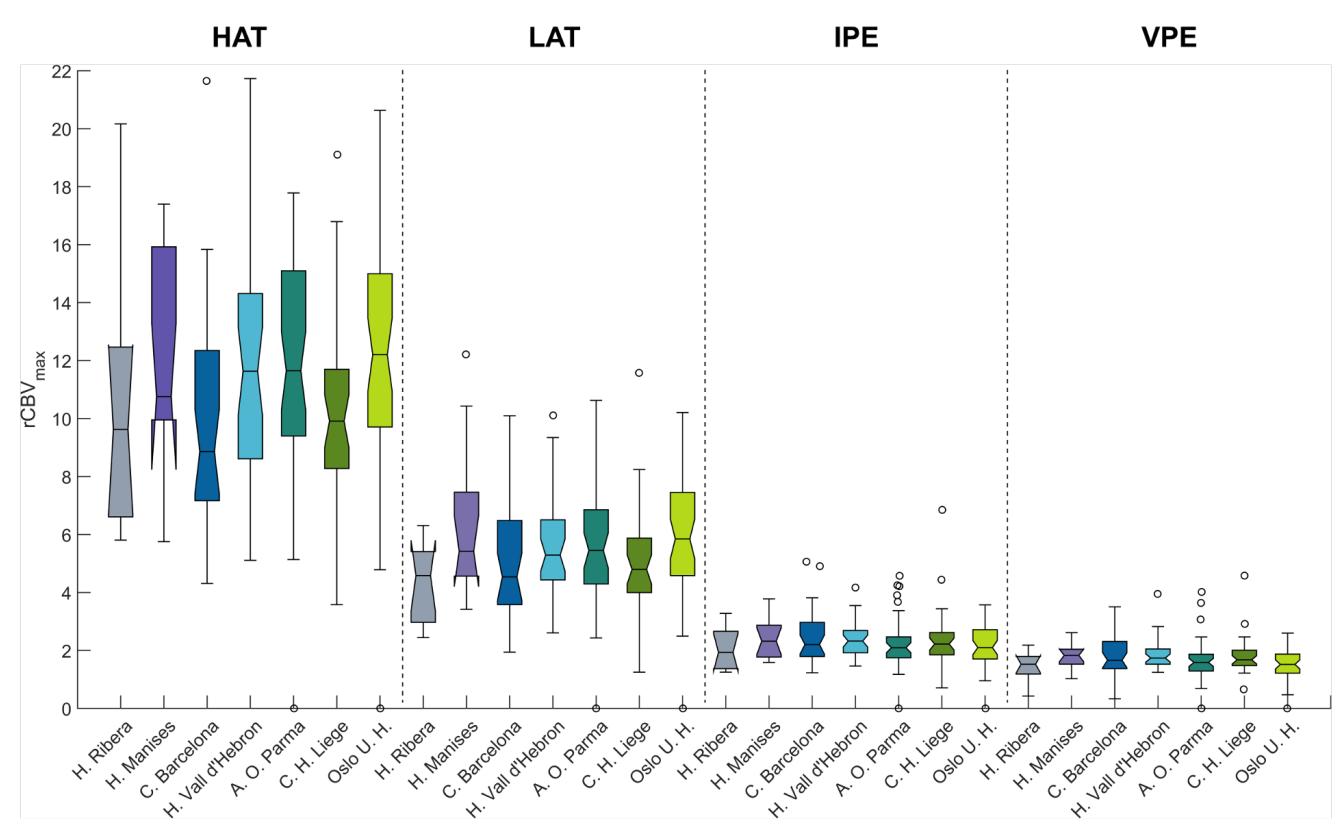

Figure 6.2: Box-whisker plots per center of the $r C B V_{\max }$ distributions at HAT, LAT, IPE and VPE habitats.

Table 6.10 shows the results of the Cox regression analysis grouped per hospital, to investigate the association of the HTS markers with patient OS at each center. Due to the small sample sizes of some centers, confidence intervals are wider, so the results of this analysis are more uncertain. However, overall, results are consistent with those obtained in the whole cohort study, consolidating the significant association between HTS habitats and patient OS in highly heterogeneous scenarios.

Figure 6.3 shows a diagram of the HRs and confidence intervals of the HAT, LAT and IPE habitats per center, and using all the data together in the analysis. The figure shows a significant overlap between confidence intervals for most of the centers, suggesting no significant differences among them, and consolidating the results obtained in the whole cohort study.

Figure 6.4 show the Kaplan-Meier estimated survival functions per center, dividing the population of each center in high and low $r C B V_{\max }$ values at HAT, LAT and IPE habitats according to the optimal thresholds obtained with the C-index method.

\subsection{Discussion}

In this work we have conducted an international multi-center validation of the HTS method published in (Juan-Albarracín et al, 2018). Using data from seven European centers, significant negative associations have been found between patient OS and the 
Table 6.10: Cox regression analysis for $r C B V_{\max }$ at each HTS habitat and patient OS per center.

\begin{tabular}{|c|c|c|c|c|c|c|c|c|}
\hline \multirow[b]{2}{*}{$r C B V_{\max }$} & & H. Ribera & H. Manises & C. Barcelona & H. Vall d'Hebron & AO Parma & CH Liege & Oslo UH \\
\hline & & & & & & & & \\
\hline \multirow{2}{*}{ HAT } & HR & 1.00 & 1.04 & 1.10 & 1.10 & 1.07 & 0.98 & 1.09 \\
\hline & $\mathrm{CI}$ & {$[0.84,1.20]$} & {$[0.96,1.13]$} & {$[0.96,1.28]$} & {$[0.98,1.23]$} & {$[0.98,1.18]$} & {$[0.86,1.12]$} & {$[1.00,1.20]$} \\
\hline \multirow{2}{*}{ LAT } & $\mathrm{HR}$ & 1.08 & 1.11 & 1.07 & 1.33 & 1.11 & 0.96 & 1.15 \\
\hline & $\mathrm{CI}$ & {$[0.64,1.81]$} & {$[0.89,1.37]$} & {$[0.79,1.44]$} & {$[0.98,1.80]$} & {$[0.95,1.30]$} & {$[0.77,1.28]$} & {$[0.97,1.36]$} \\
\hline \multirow{2}{*}{ IPE } & $\mathrm{HR}$ & 1.95 & 1.76 & 1.01 & 1.73 & 1.40 & 1.10 & 1.54 \\
\hline & $\mathrm{CI}$ & {$[0.5,7.65]$} & {$[0.80,3.89]$} & {$[0.61,1.65]$} & {$[0.65,4.61]$} & {$[0.97,2.01]$} & {$[0.64,1.90]$} & {$[0.92,2.57]$} \\
\hline \multirow{2}{*}{ VPE } & $\mathrm{HR}$ & 2.13 & 1.83 & 0.92 & 1.20 & 1.31 & 1.11 & 1.67 \\
\hline & CI & {$[0.12,35.9]$} & {$[0.42,7.89]$} & {$[0.39,2.18]$} & {$[0.39,3.68]$} & {$[0.81,2.13]$} & {$[0.45,2.67]$} & {$[0.80,3.48]$} \\
\hline \# patients & & 7 & 14 & 25 & 33 & 40 & 33 & 32 \\
\hline
\end{tabular}

CI Indicates a Confidence Interval.
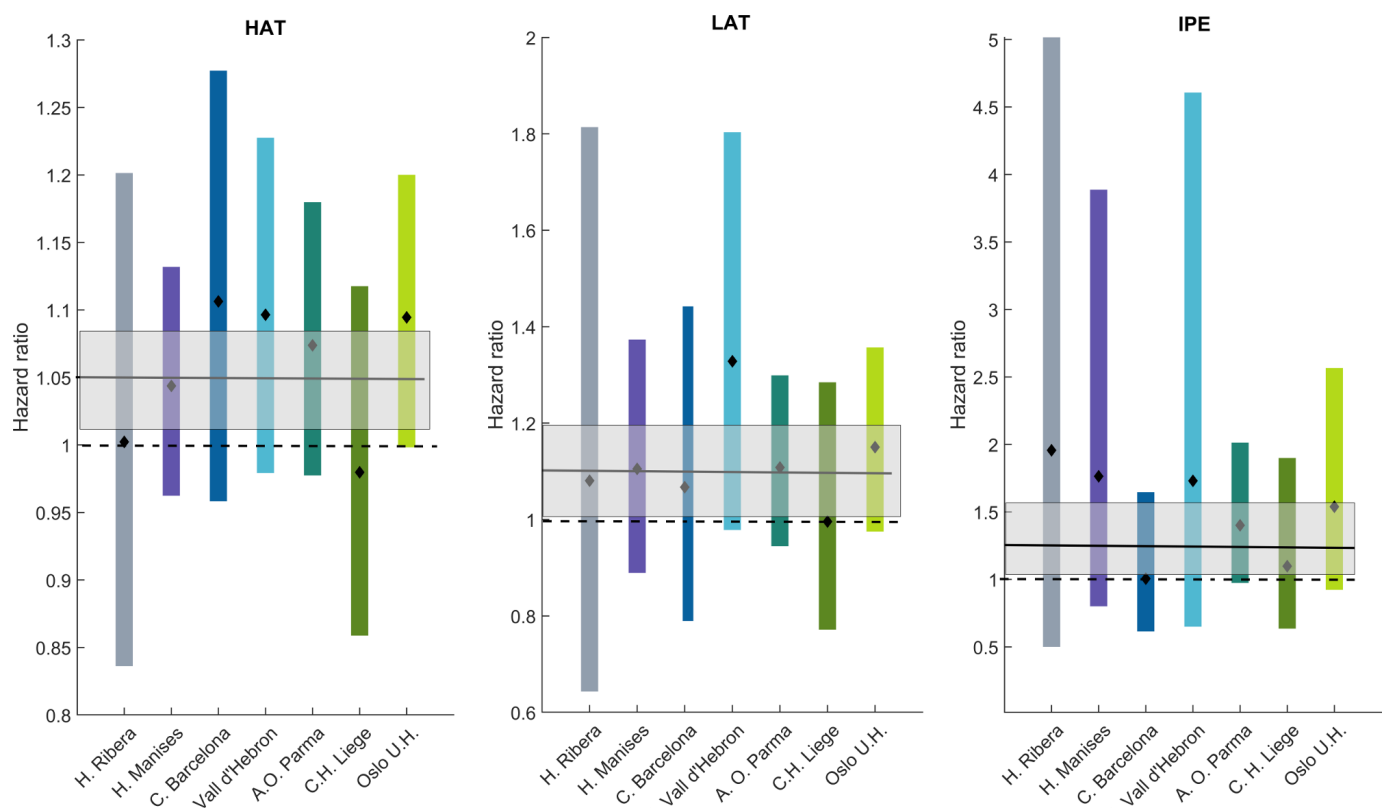

Figure 6.3: Diagram with the HRs and 95\% confidence intervals per center, to investigate the association between OS and HTS markers at HAT, LAT and IPE habitats. The continuous black lines and the grey bands correspond to the HRs and their associated confidence intervals using the data from all centers. Black diamond markers and colored bars represent the HR with its confidence interval for each respective center.

HTS markers at HAT, LAT and IPE habitats, consolidating the results obtained in the aforementioned single-center study conducted by Juan-Albarracín et al (2018).

Addressing heterogeneity between centers in the estimation of MRI markers is not an easy task. Several authors in the bibliography have pointed out the uncertainty and low reproducibility of MRI markers, especially across multiple centers (Abramson et al, 2015; O'Connor et al, 2017; Schnack et al, 2004; De Guio et al, 2016). The nonquantitative nature of several MRI acquisitions and the manual procedure for obtaining MR-based biomarkers introduce important sources of variability, making it difficult to 
Chapter 6. Multi-center international validation of the Hemodynamic Tissue Signature for glioblastoma
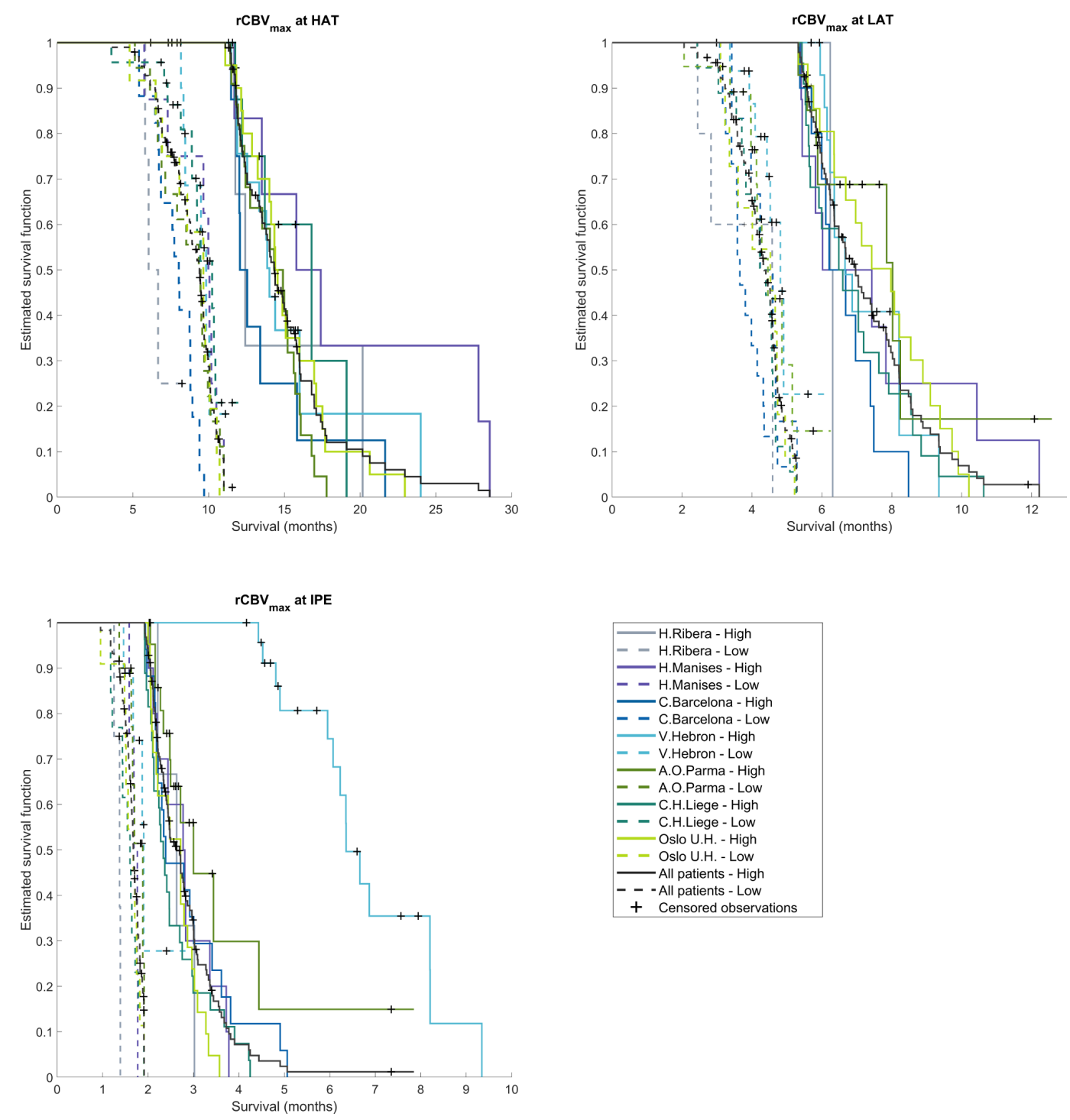

Figure 6.4: Kaplan-Meier estimated survival functions for the populations of each center stratified into groups according to high or low $r C B V_{\max }$ at HAT (top left), LAT (top right) and IPE (bottom left), using the optimal thresholds calculated with the C-index method. 
validate new robust and stable MRI markers (Schnack et al, 2004).

The HTS method focuses its efforts on the automated delineation of habitats related to perfusion patterns within the lesion in a robust and reliable manner. In the current study, a cohort with large variations in terms of patient demographics as well as MRI acquisition protocols was used to measure the robustness of the method. The experiments conducted in our study did not show relevant differences among the distributions of the HTS markers obtained from MRI studies acquired at the different centers. Only for a small number of cases, significant differences were found for the HAT and LAT markers among centers. These results strongly suggest that the HTS method is robust against inter-center variability in the task of describing the vascular heterogeneity of the glioblastoma. Furthermore, the results of the Cox and KaplanMeier analyses per center showed robust associations between patient OS and the HTS markers, regardless of the center of origin. The proposed thresholds were also effective in stratifying patients from different centers into low- and high-vascular groups, presenting different OS tendencies.

Consistently with the literature, the HTS method strongly correlates with patient OS when observing the $r C B V_{\max }$ values at HAT habitat. Such values represent the most hyper-perfused measures of the glioblastoma, which aligns with the measurements proposed in previous studies in the literature (Jain et al, 2014; Liu et al, 2017a; Hirai et al, 2008). As expected, shorter OS survival rates were found for patients with higher $r C B V_{\text {max }}$ values at the HAT habitat. Similarly, we also found that LAT habitat present strong association with OS and high stratification abilities. Both results have been replicated under highly variable conditions of MRI acquisition protocols and patient demographics, hence demonstrating the robustness of the HTS method in describing the vascular arrangement of the glioblastoma.

One of the most important finding presented in Juan-Albarracín et al (2018) was the correlation between long-term OSs and lower $r C B V_{\max }$ values in the IPE habitat. The peritumoral region of the glioblastoma is the most heterogeneous area of the tumor, in which uncontrolled infiltration occurs. Moreover, the inter-patient variability and the inter-center heterogeneity significantly increases the uncertainty in this region, obscuring the important information that it contains. However, in the present study we found statistical association between perfusion markers at this region and patient OS, even under the large heterogeneous nature of the proposed cohort. Moreover, effective stratification capabilities were also found when employing this marker as indicator to divide the population between high- and low-vascular glioblastomas.

Having demonstrated the influence of early-stage vascularity on the prognosis of glioblastoma, we suggest the use of this factor in any clinical study that includes population randomization. Authors consider that the HTS method will help overcome current limitations and improve patient recruitment and randomization by initiating a route map to avoid the second translational gap cited previously in (Abramson et al, 2015).

One of the most important limitations of our study is the imbalance between the number of patients in each center. Although the whole cohort size is large enough for a powerful statistical study, some of the participating centers provided a low number of patients (less than 15 patients), which introduces limitations and uncertainties into the 
Chapter 6. Multi-center international validation of the Hemodynamic Tissue Signature for glioblastoma

studies conducted per center. On the other hand, since the influence of the molecular markers in patient prognosis has been clearly demonstrated (Louis et al, 2016; Verhaak et al, 2010), it may be of interest to add them as co-factors in the regression survival models. In future studies we plan to analyze the possible association between molecular and imaging markers and their prognostic possibilities. 


\section{Chapter 7}

\section{ONCOhabitats: A system for glioblastoma heterogeneity assessment through Magnetic Resonance Imaging}

Neuroimaging analysis is currently crucial for an early assessment of glioblastoma, to help improving treatment and tumor follow-up. To this end, multiple quantitative and morphological MRI sequences are usually employed, requiring the development of automated tools capable to extract the relevant information contained in these sources. Despite major advances in MRI brain tumor technology, the latter is generally private and inaccessible to the research community. This significantly slows down the advances in tumor understanding, as many researchers continually have to re-implement many software pieces for the typical MRI analysis pipeline to conduct their investigation. This task is often arduous or even unreachable to many research groups specialized on the clinical aspects of the tumor, and with less knowledge about state-of-the-art technology for MRI analysis. As a result, many efforts are lost in developing this necessary technology before research begins.

In this thesis, several methods have been developed to analyze glioblastoma through MRI. One of the aspects significantly taken into consideration since the beginning of this thesis was to, parallel to the academic and theoretical research, develop the required infrastructure to facilitate public access to the technology developed in the thesis. In this sense, this chapter presents ONCOhabitats (https://www.oncohabitats. upv.es): an online open access system for glioblastoma heterogeneity assessment by MRI data. ONCOhabitats provides two services for untreated glioblastomas: 1) malignant tissue segmentation, and 2) vascular heterogeneity assessment of the tumor. The segmentation service was validated against the BRATS 2017 reference dataset, showing comparable results with current state-of-the-art methods (WT Dice score: 0.89). The vascular heterogeneity assessment service was validated in a retrospective cohort of 50 patients, in a study focused on predicting patient OS. Cox proportional hazard regression analysis and Kaplan-Meier survival study showed significant positive correlations ( $\mathrm{p}$-value $<.05$ ) between the HTS habitats and patient OS. ONCOhabitats 
system also generates radiological reports for each service, including volumetries and perfusion measurements of the different regions of the lesion. Additionally, ONCOhabitats gives access to the scientific community to a computational cluster capable to process about 300 cases per day.

The contents of this chapter were published in the journal publication (Juan-Albarracin et al, 2019b) - thesis contributions C6 and P4, P8, P9 and P10.

\subsection{Introduction}

Glioblastoma is a primary brain tumor presumed to arise from neuroglial cells. It is the most malignant and frequent astrocytoma, accounting for more than $60 \%$ of all brain tumors in adults. Glioblastoma has a global incidence of 4.67 to 5.73 per 100,000 people, and presents a poor prognosis of 14-15 months under the best treatment (Stupp et al, 2005).

Heterogeneity is a hallmark that has been identified as crucial to understand the tumor aggressiveness and its resistance against therapies (Lemée et al, 2015; Soeda et al, 2015). Specifically, glioblastoma is characterized by a high heterogeneity both at macroscopic tissue level, with co-existence of different malignant tissues within the neoplasm (Liu et al, 2017b); as well as at microscopic cellular level, with different molecular sub-types and genetic alterations (Inda et al, 2014). Such heterogeneity rises this tumor as one of the deadliest malignant primary brain tumor in adults (Ostrom et al, 2015).

Molecular analysis of glioblastoma has largely improved the understanding of the biological heterogeneity of these tumors. Molecular profiling of glioblastoma has allowed the identification of different tumor sub-types, helping in the development of more efficient drugs (Parsons et al, 2008; Verhaak et al, 2010). However, in the past years, significant interest has been placed in the analysis of glioblastoma heterogeneity based on medical imaging, to discover non-invasive tumor features related to different outcomes such as overall survival, tumor grading or glioblastoma molecular sub-typing (Wangaryattawanich et al, 2015).

Characterization of glioblastoma heterogeneity based on MRI has been addressed from a wide range of approaches. Glioblastoma tissue segmentation has gathered most of these efforts. Automated identification of the different tissues that co-exist in the lesion, such as enhancing tumor, non-enhancing tumor, edema and necrosis, has been largely addressed by the scientific community. This has lead to initiatives such as the BRATS challenge, which was born in 2012 and has become the reference benchmark (Menze et al, 2015) to evaluate the state-of-the-art of automated high-grade and low-grade glioma segmentation algorithms. Nowadays, with the advent of novel deep learning techniques, the current state-of-the art is mostly dominated by CNN classifiers. CNNs are a class of deep feed-forward neural networks whose architecture is particularly well suited for computer vision recognition tasks. In medical image analysis field, CNNs have outperformed most of algorithms in many problems, arising as the winner technique in most challenges such as BRATS, ISLES or PROMISE12 challenges (Crimi et al, 2016, 2017, 2018). 
In addition to glioblastoma tissue segmentation, PWI has played a key role in the advanced characterization of the tumor heterogeneity based on MRI (Shah et al, 2010; Lupo et al, 2005; Knopp et al, 1999). Glioblastoma is characterized by a robust angiogenesis, strong vascular proliferation and an aberrant microvasculature (Alves et al, 2011; Hardee and Zagzag, 2012; Kargiotis et al, 2006). Numerous studies have focused on the analysis of perfusion indices to assess tumor grading (Law et al, 2003; Emblem et al, 2008), early response to treatment assessment (Elmghirbi et al, 2017; Vidiri et al, 2012), recurrence vs radionecrosis (Hu et al, 2009; Barajas et al, 2009) or clinical outcome prediction (Mangla et al, 2010; Jain et al, 2014). More recent studies addressed the local characterization of sub-regions within the glioblastoma using DSC, DCE or Magnetic Resonance Spectroscopy Imaging (MRSI) (Artzi et al, 2014; Akbari et al, 2014; Sawlani et al, 2010; Raschke et al, 2019). Specifically, in (Juan-Albarracín et al, 2018) an unsupervised method called HTS was proposed to characterize the vascular heterogeneity of glioblastoma based on DSC. This method combined perfusion biomarkers and glioblastoma tissue segmentation to discover habitats within the neoplasm, showing significant correlation with patient overall survival.

However, despite the great advances in novel methods to describe glioblastoma heterogeneity, most of them are based on private algorithms and in-house technology developed by the authors, non-accessible for the scientific community. MRI-dedicated libraries such as Advanced Normalization Tools (ANTs) (Avants et al, 2011; Tustison et al, 2014), FSL (Jenkinson et al, 2012) or ITK (Avants et al, 2014), as well as modern toolkits for deep learning such as Tensorflow ${ }^{T / 4}$ or PyTorch, are provided to develop such technologies. However, these libraries are just the pieces to build the complex stateof-the-art models to analyze glioblastoma, whose development requires considerable efforts, resources and arduous learning curves, which are not often accessible to many researchers or institutions. In this regard, open-access public platforms that implement state-of-the-art techniques in a user-transparent manner are highly desirable to bring to the scientific community the possibility to conduct advanced multiparametric analysis of glioblastoma.

In this work we present ONCOhabitats: an online system aimed to provide state-ofthe-art analysis services for glioblastoma. ONCOhabitats provides two main services for untreated glioblastoma: 1) High-grade glioma tissue segmentation based on CNN; and 2) glioblastoma vascular heterogeneity assessment by means of the HTS method proposed in (Juan-Albarracín et al, 2018). For each service, ONCOhabitats returns the preprocessed images, the tissue segmentation and habitats maps, and automatically generates a radiological report summarizing all the findings of the study.

\subsection{Materials}

To validate ONCOhabitats technology several datasets were employed for the different services.

ONCOhabitats high-grade glioma segmentation service was evaluated with the public BRATS 2017 challenge dataset, provided for the international MICCAI 2017 conference. The training corpus of the BRATS 2017 dataset consists of multi-parametric MR scans of 210 high-grade gliomas: 20 patients from the BRATS 2013 dataset, 88 
from the Center for Biomedical Image Computing and Analytics (CBICA) and 102 from the The Cancer Imaging Archive (TCIA) corpus. The validation corpus consists of 46 multi-contrast MR scans of high-grade glioma patients distributed in 16 cases from the CBICA institution, 24 cases from the TCIA corpus and 6 cases from the University of Alabama at Birmingham (UAB) department.

For each patient, pre- and post-gadolinium $\mathrm{T}_{1}$-weighted, $\mathrm{T}_{2}$-weighted and FLAIR MR exams were provided. All images were linearly co-registered to the post-gadolinium $\mathrm{T}_{1}$-weighted exam, skull stripped, and interpolated to $1 \mathrm{~mm}^{3}$ isotropic resolution.

Manual expert annotations of this dataset comprise 4 classes: Class 1) necrosis, cyst, hemorrhage and non-enhancing tumor; class 2) surrounding edema; class 4) enhancing tumor core; and class 0) for everything else. Evaluation is assessed for 3 different compartments, whose composition is shown in table 7.1.

Table 7.1: Labels composing each sub-compartment evaluated in the BRATS 2013 challenge.

\begin{tabular}{ccccc}
\hline & Label 0 & Label 1 & Label 2 & Label 4 \\
WT & $\times$ & $\times$ & $\times$ \\
TC & & $\times$ & & $\times$ \\
ET & & & & $\times$ \\
\hline
\end{tabular}

The glioblastoma vascular heterogeneity service was validated with a retrospective local dataset of 50 patients, including 33 men with an average age of 60.94 years (range, 25-80 years) and 17 women with an average age of 62.53 years. MRI included preand post-gadolinium $\mathrm{T}_{1}$-weighted, $\mathrm{T}_{2}$-weighted and FLAIR MR exams, and DSC $\mathrm{T}_{2^{*}}$ weighted perfusion study. The institutional review board approved this retrospective study, and the requirement for patient informed consent was waived. The patient inclusion criteria and MRI protocol are extensively detailed in section 5.2.

\subsection{Methods}

ONCOhabitats provides two main services: 1) High-grade glioma segmentation, and 2) glioblastoma vascular heterogeneity assessment.

Figure 7.1 shows an outline of the different sub-processes involved in each service. The first pipeline performs a morphological segmentation of high-grade glioma tumors by first pre-processing the MRI and then using a 3D U-Net CNN classifier. The second pipeline extends the morphological segmentation pipeline by incorporating DSC perfusion pre-processing and quantification, and the HTS method to detect regions within the glioblastoma with different hemodynamic activity.

We will first describe both services and then the ONCOhabitats on-line system will be presented. 


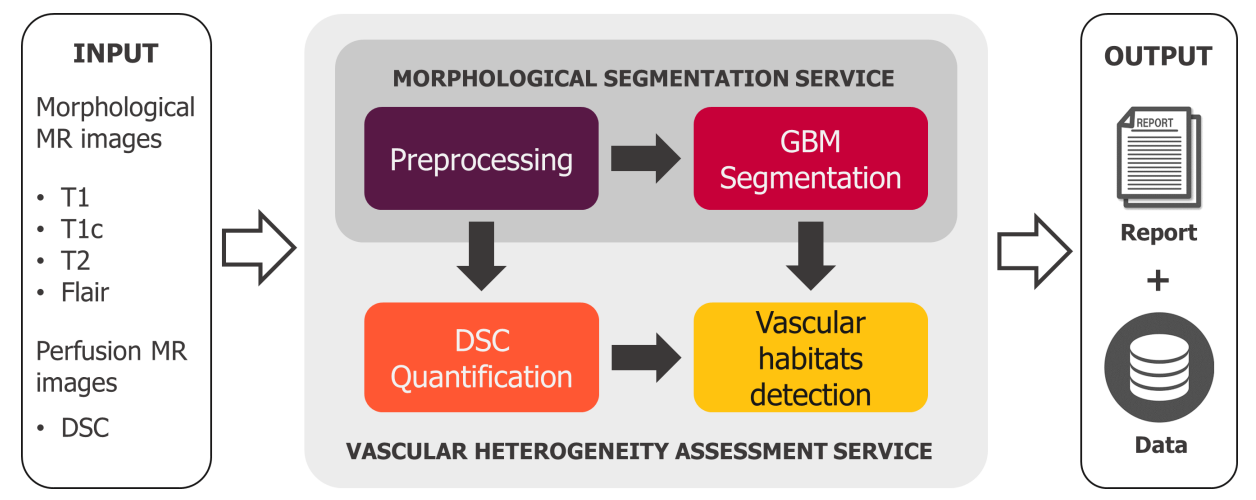

Figure 7.1: Diagram of the different modules comprising the high-grade glioma segmentation and glioblastoma vascular heterogeneity assessment services of ONCOhabitats.

\subsubsection{High-grade glioma segmentation service}

ONCOhabitats considers three tissues to morphologically describe high-grade gliomas: 1) enhancing tumor, 2) edema and 3) necrotic and non-enhancing regions of the tumor. The service is composed of two stages: 1) MRI preprocessing and 2) Segmentation based on CNNs.

\section{MRI preprocessing}

Our preprocessing module includes the following steps: (1) voxel isotropic resampling of all MR images, (2) denoising, (3) rigid intra-patient MRI registration, (4) affine registration of all sequences to Montreal Neurological Institute (MNI) ICBM space, (5) skull-stripping and (6) magnetic field inhomogeneity correction. Voxel resampling is performed at $1 \mathrm{~mm}^{3}$ by means of linear interpolation. Denoising is carried out using the adaptive non local means filter (Manjón et al, 2010c) with a search window of $7 \times 7 \times 7$ voxels and a patch window of $3 \times 3 \times 3$ voxels. Registration is conducted with the ANTs software (Avants et al, 2008), taking the $T_{1 C E}$ sequence as reference and using Mutual Information metric. In a previous version of ONCOhabitats, skullstripping was performed with an in-house pipeline based on a non-linear registration of the $\mathrm{T}_{1 C E}$ sequence to a template with a known intra-cranial mask. Nowadays, skullstripping if performed with a patch-based U-net CNN working with $\mathrm{T}_{1 C E}$ patches of $32 \times 32 \times 32$ trained on 120 manually segmented glioblastomas. The network has a performance of 0.94 Dice score on an independent test set. Finally, magnetic field inhomogeneities are corrected with the N4 software using the previously computed intra-cranial mask (Tustison et al, 2010).

\section{High-grade glioma segmentation}

ONCOhabitats CNN takes as input the $\mathrm{T}_{1 C E}, \mathrm{~T}_{2}$ and FLAIR MRI and works with $3 \mathrm{D}$ patches of size $32 \times 32 \times 32$. We followed a U-net architecture (Ronneberger et al, 2015; Soltaninejad et al, 2018; Dong et al, 2017) of 5 levels, with a contracting and expanding paths of 4 residual-blocks preceded of 4 simple-blocks. A simple-block consists of the following sequence of operations: convolution + batch normalization + 
ReLu activation function, while a residual-block implements the proposal of He et al (He et al, 2016): convolution + batch normalization + ReLu + convolution + batch normalization + residual connection + ReLu activation function. Max-pooling of size 2 is employed to down-sample patches at each level, while transpose convolutions are employed to up-sample patches in the expanding path. The number of filters per level are: 16 at first level (native patch resolution of $32 \times 32 \times 32$ ), 32 filters at second level (patch resolution of $16 \times 16 \times 16$ ), 64 filters at third level (patch resolution of $8 \times 8 \times 8$ ), 128 filters at fourth level (patch resolution of $4 \times 4 \times 4$ ) and 256 filters at fifth level (patch resolution of $2 \times 2 \times 2$ ). Long-term concatenations are also employed to connect blocks at each level.

Isotropic kernels of $3 \times 3 \times 3$ were employed for all convolutions. The network was trained using Adam Optimizer with an initial learning rate of $1 e-3$ and cross-entropy was used as loss function. $L 2$ regularization with penalty $1 e-3$ was employed to avoid for over-fitting. We employed a batch size of 64 individuals, forcing an equal representation of enhancing tumor, edema, necrosis and healthy patches to compensate for class imbalance. The network was trained for $50 k$ iterations.

Figure 7.2 summarizes the network architecture and the internal design of the simple and residual blocks.

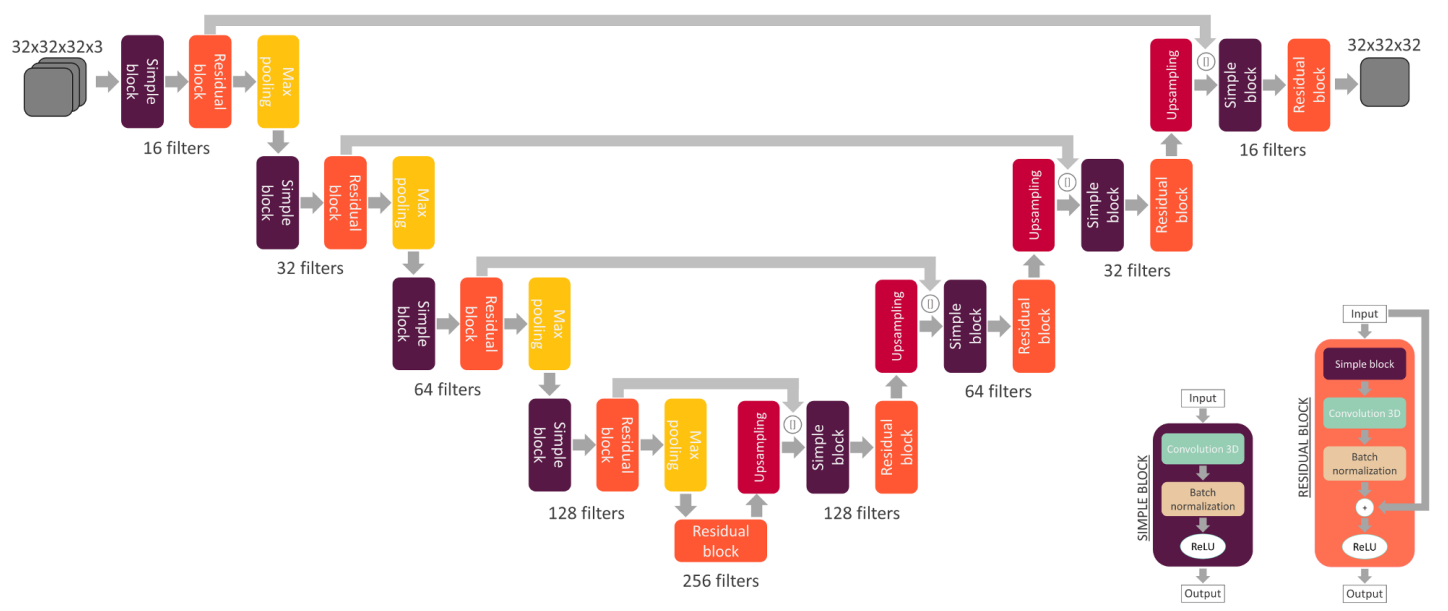

Figure 7.2: High-grade glioma Convolutional Neural Network architecture for the ONCOhabitats morphological segmentation service.

\subsubsection{Glioblastoma vascular heterogeneity assessment service}

This service extends the morphological segmentation service by introducing perfusion information into the study. The service implements the HTS method presented in (Juan-Albarracín et al, 2018), which aims to describe the vascular heterogeneity of glioblastoma. The HTS combines the glioblastoma morphological segmentation with perfusion indexes such as $\mathrm{rCBV}$ and $\mathrm{rCBF}$ to discover habitats within each tissue with different patterns of vascularity. We found that these habitats provide relevant information to early predict patient survival, even taking into account the variations in treatment. 
The service includes four stages: 1) MRI preprocessing, 2) Segmentation based on CNNs, 3) Perfusion quantification and 4) Vascular habitats detection. The MRI preprocessing and glioblastoma segmentation are inherited from the high-grade glioma segmentation service.

\section{Perfusion quantification}

ONCOhabitats rCBV and rCBF maps are quantified by means of standard techniques proposed in the literature (Knutsson et al, 2010). For a detailed explanation of the calculation of perfusion parametric maps, please refer to section 2.2.2. In order for this chapter to be self-contained, a short remainder will be made.

$\mathrm{T}_{1}$-weighted leakage effects are automatically corrected using the Boxerman method (Boxerman et al, 2006), while gamma-variate curve fitting is employed to correct for $\mathrm{T}_{2}$ extravasation phase. $\mathrm{rCBV}$ is computed by numerical integration of the area under the gamma-variate curve (Knutsson et al, 2010), while rCBF is calculated based on the block-circulant SVD devolution technique proposed in (Wu et al, 2003). The AIF is automatically calculated using a divide and conquer algorithm, which recursively dichotomizes the gadolinium concentration-time curves into two groups, selecting those curves with higher peak height, earliest time to peak and quickest wash-out (i.e. lowest full width at half maximum). The AIF is finally computed as the average of the curves of the final group that contains 10 or fewer curves.

\section{Vascular habitats detection}

The HTS method (Juan-Albarracín et al, 2018) describes the vascular heterogeneity of the glioblastoma by means of an unsupervised analysis of the perfusion patterns detected within the lesion. Such analysis is designed to yield four habitats: the HAT, the LAT, the potentially IPE and the VPE.

The unsupervised analysis of perfusion patterns is carried out through the DCMSVFMM algorithm (Sfikas et al, 2008) (with DCAGMRF prior (Nikou et al, 2007)). Such algorithm is an extension of the classic FMM specially focused on image data, which incorporates a continuous MRF on the spatial coefficients of the model to capture the self-similarity and local redundancy of the images. The HTS method consists of two stages: (a) an initial re-definition of the enhancing tumor and edema ROIs obtained by the morphological segmentation using perfusion information, and (b) a cluster analysis of the perfusion heterogeneity within each previously mentioned ROI to detect the different vascular behaviors expressed by the neoplasm. A comprehensive detailed explanation of the HTS method is performed in section 5.3.3.

\subsubsection{Clinical report}

Clinical reports are automatically generated after the finalization of each ONCOhabitats job. The reports summarize all the findings of the studies, including morphological and functional measurements of each glioblastoma tissue and habitat. Regarding the morphological segmentation service jobs, absolute tissue volumetry in $\mathrm{cm}^{3}$, as well as relative volumetry with respect to the intra-cranial cavity are calculated for the 
enhancing tumor, necrosis and edema tissues. Concerning the vascular heterogeneity assessment service jobs, in addition to the tissue volumetries, habitat's absolute and relative volumetries are also calculated. Moreover, a tendency analysis of the perfusion biomarkers confined within each tissue and habitat is also included in the report. Median perfusion values of each ROI as well as Median Absolute Deviations are calculated to robustly determine the vascular tendency of each sub-comparment of the lesion. Finally, perfusion prototypical curves in combination with a radar chart of the perfusion biomarkers at each region are also included in the report for a visual representation of the functional behavior of the glioblastoma. Figures ?? show an example of a vascular heterogeneity assessment report.

\subsubsection{ONCOhabitats system}

ONCOhabitats is a web service solution to carry out the previously presented analyses. The platform implements a Software as a Service (SaaS) model to automatically analyze glioblastoma cases. Figure 7.8 shows the diagram scheme of ONCOhabitats system.

The system implements a Wordpress ${ }^{\circledR}$ landing web-page as front-end for the user. Before applying for a job, user must be registered in ONCOhabitats system. Registration requires a username, the first and last names, the institution of provenance and a valid email only used to inform the user about the status of their jobs. After registration, the user can upload the MR images to a data storage secure server to launch the jobs.

The system implements secure encrypted communication via HTTPS protocol with a trusted certificate to enhance data protection and privacy. The data storage includes file encryption and secure transfer protocol via SFTP. ONCOhabitats currently supports DICOM and NIfTI (compressed and uncompressed) medical imaging formats. An automated de-identification is carried out for all DICOM files using the gdcmanon tool from the Grassroots DICOM library. Once DICOM files are de-identified, they are converted to compressed NIfTI format and automatically removed from the server. The de-identified NIfTI files are stored in a secure server, non-accessible through the ONCOhabitats website, to enhance security and data protection. Once the analysis is complete, the pre-processed images as well as the resulting segmentation masks and biomarker maps are stored in a separated data storage, only accessible for 15 days by the user owner of the job. After this period, all the data is completely removed from the ONCOhabitats servers, unless the user explicitly specifies through his account web-page that his data can be used for research purposes, in which case the data is kept on a private server. This procedure follows the guidelines recommended by the Data Protection Officer (DPO) of the Universitat Politècnica de Valencia (UPV) and has been approved by the ethical committee of our institution.

ONCOhabitats system equips 7 DELL PowerEdge R720 ${ }^{\circledR}$ dedicated servers, each one shipping two Intel Xeon E5-2620 CPUs with a total of 12 cores and 64 GB of RAM. Two NVidia Titan Xp with 3840 CUDA $^{\circledR}$ cores and 12 GB of RAM supports the cluster for the deep learning tasks.

The ONCOhabitats pipelines are mostly implemented in $\mathrm{C}++$ using ITK and Eigen 


\section{ONCOhabitats}

\section{Vascular}

heterogeneity

\section{assessment report}

www.oncohabitats.upv.es

Biomedical Informatics Group

ITACA Institute, Universitat Politècnica de València

Report Number 5d202f80c30ee781982602b88bec 859549 bface 7
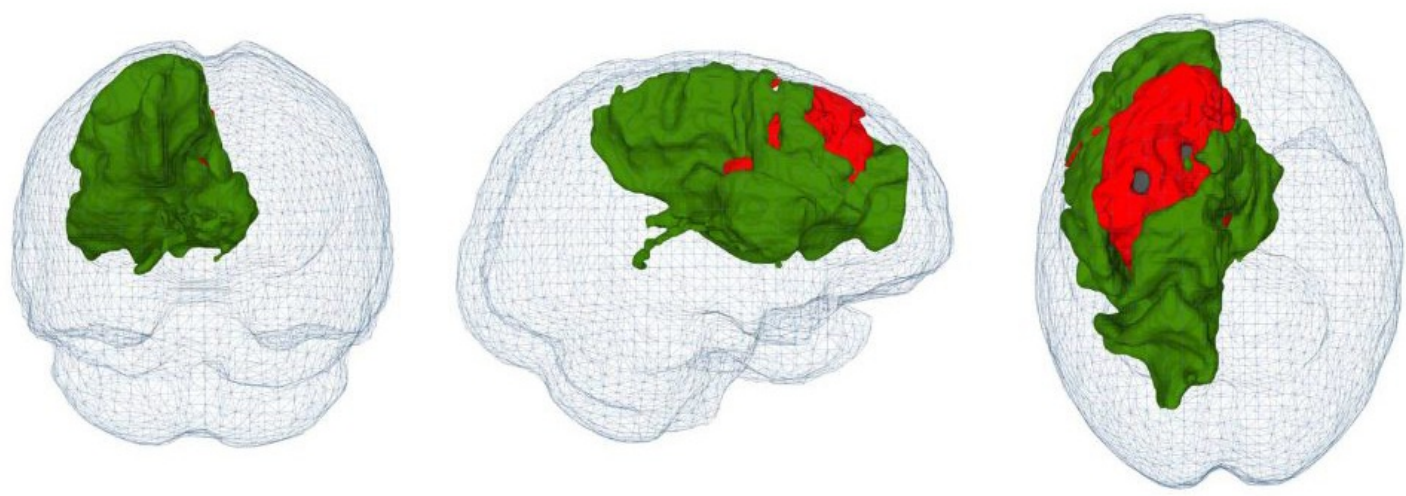

Figure 7.3: Example of the first page of a report for a vascular heterogeneity assessment analysis of a glioblastoma. 
Chapter 7. ONCOhabitats: A system for glioblastoma heterogeneity assessment through Magnetic Resonance Imaging

ONCOhabitats - Vascular Heterogeneity Assessment Report

\begin{tabular}{|c|c|c|}
\hline \multicolumn{3}{|l|}{ STUDY INFORMATION } \\
\hline \multirow{2}{*}{\multicolumn{2}{|c|}{$\begin{array}{l}\text { Report ID } \\
5 d 202 f 80 c 30 e e 781982602 b 88 \mathrm{bec} 859549 \mathrm{bface} 7\end{array}$}} & Report Date \\
\hline & & $20-N o v-2019$ \\
\hline User Name & Institution & E-mail \\
\hline ONCOhabitats ONCOhabitats & ONCOhabitats & javij1@gmail.com \\
\hline Patient ID & Patient Gender & Patient Age \\
\hline W30 Ivy Gap & Male & 60 \\
\hline
\end{tabular}

- Volumetry $\left(\mathrm{cm}^{3}\right)$

\begin{tabular}{|c|c|c|c|c|c|c|c|}
\hline Intra-crar & cavity & Enhan & Tumor & Edema & & Necros & \\
\hline Abs. & Rel. & Abs. & Rel. & Abs. & Rel. & Abs. & Rel. \\
\hline 1602.17 & $100.00 \%$ & 37.38 & $2.33 \%$ & 35.75 & $2.23 \%$ & 18.77 & $1.17 \%$ \\
\hline
\end{tabular}

- Intra-cranial cavity mask (axial / sagittal / coronal)
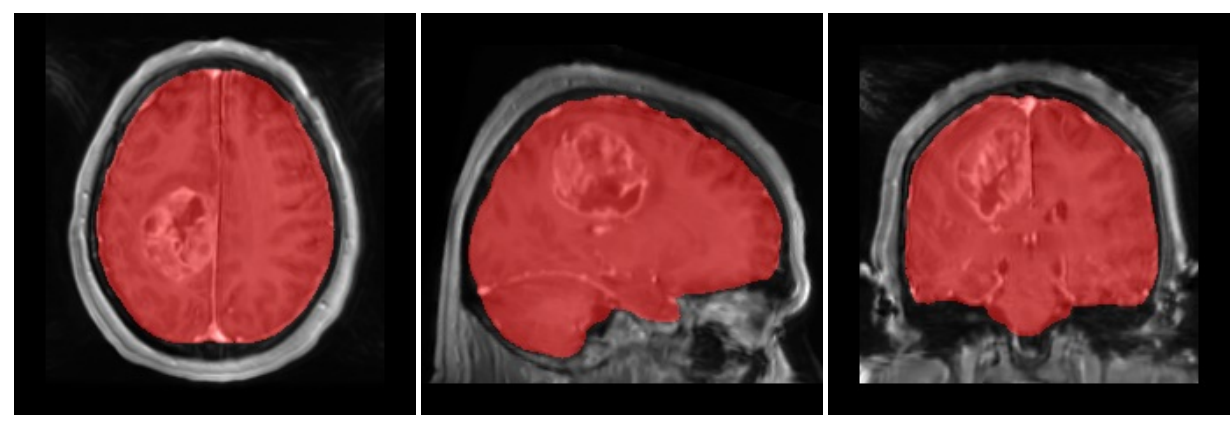

- GBM anatomical segmentation maps (axial/ sagittal / coronal)
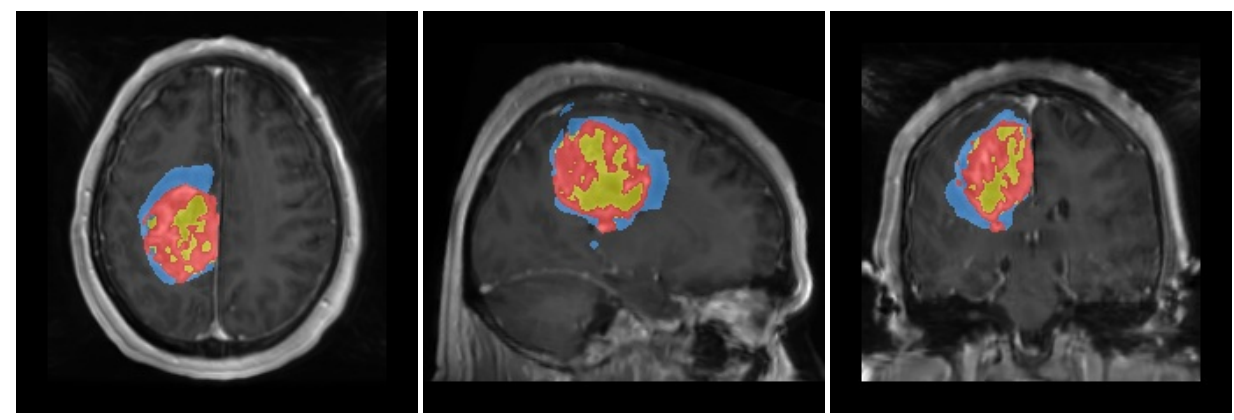

${ }^{1}$ The results contained in this report are only certified for research purposes.

- www.oncohabitats.upv.es -

Figure 7.4: Example of the second page of a report for a vascular heterogeneity assessment analysis of a glioblastoma. 
ONCOhabitats - Vascular Heterogeneity Assessment Report

\section{DSC QUANTIFICATION}

- Median and MAD deviation of DSC biomarkers per tissue (enhancing tumor, edema and necrosis)

\begin{tabular}{l|lll} 
& rCBV & rCBF & MTT \\
\hline Enh.Tumor & $4.71 \pm 1.78$ & $3.64 \pm 1.30$ & $0.14 \pm 0.01$ \\
Edema & $1.74 \pm 1.04$ & $1.56 \pm 0.80$ & $0.12 \pm 0.01$
\end{tabular}

- Biomarker maps: 1) rCBV map, 2) rCBF map, and 3) MTT map
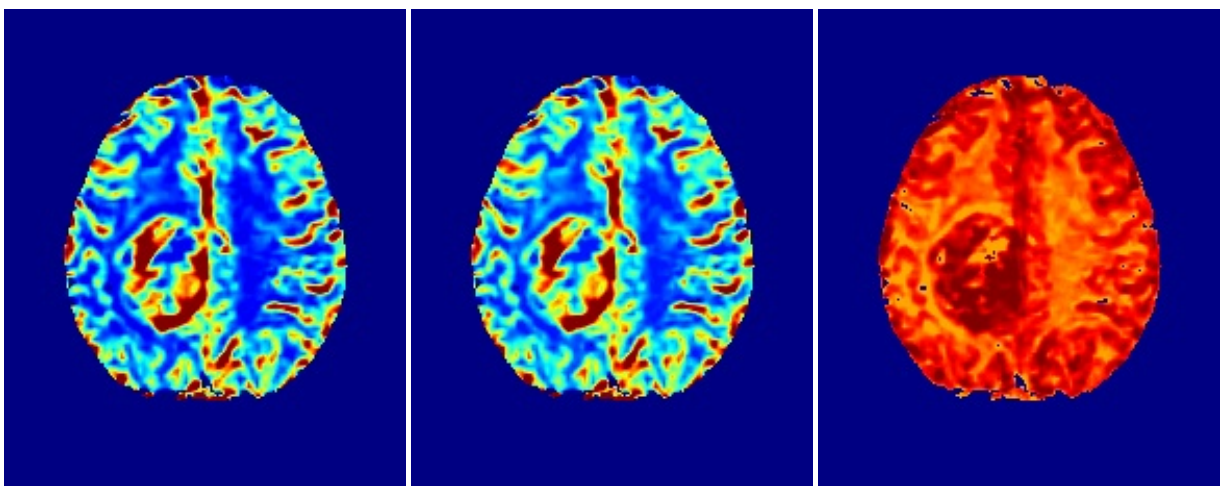

- K2 leakage correction map (Boxerman et. al 2006)

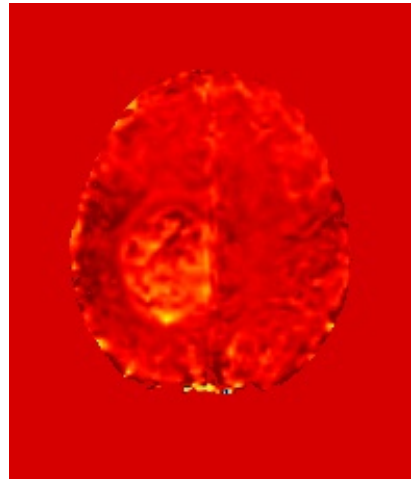

${ }^{1}$ The results contained in this report are only certified for research purposes.

Figure 7.5: Example of the third page of a report for a vascular heterogeneity assessment analysis of a glioblastoma. 
Chapter 7. ONCOhabitats: A system for glioblastoma heterogeneity assessment through Magnetic Resonance Imaging

ONCOhabitats - Vascular Heterogeneity Assessment Report

HEMODYNAMIC DSC TISSUE SIGNATURE

- Volumetry per vascular habitat $\left(\mathrm{cm}^{3}\right)$

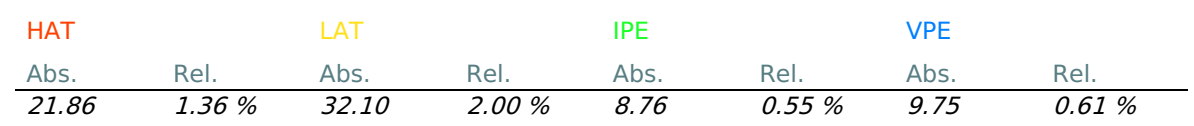

- Median and MAD deviation of DSC biomarkers per vascular habitat

\begin{tabular}{l|lll} 
& rCBV & rCBF & MTT \\
\hline HAT & $6.84 \pm 1.04$ & $5.29 \pm 0.83$ & $0.14 \pm 0.00$ \\
LAT & $3.46 \pm 0.90$ & $2.73 \pm 0.65$ & $0.14 \pm 0.01$ \\
IPE & $1.20 \pm 0.16$ & $1.16 \pm 0.13$ & $0.12 \pm 0.00$ \\
VPE & $0.64 \pm 0.15$ & $0.70 \pm 0.13$ & $0.10 \pm 0.00$
\end{tabular}

- Hemodynamic DSC tissue signature nosological map
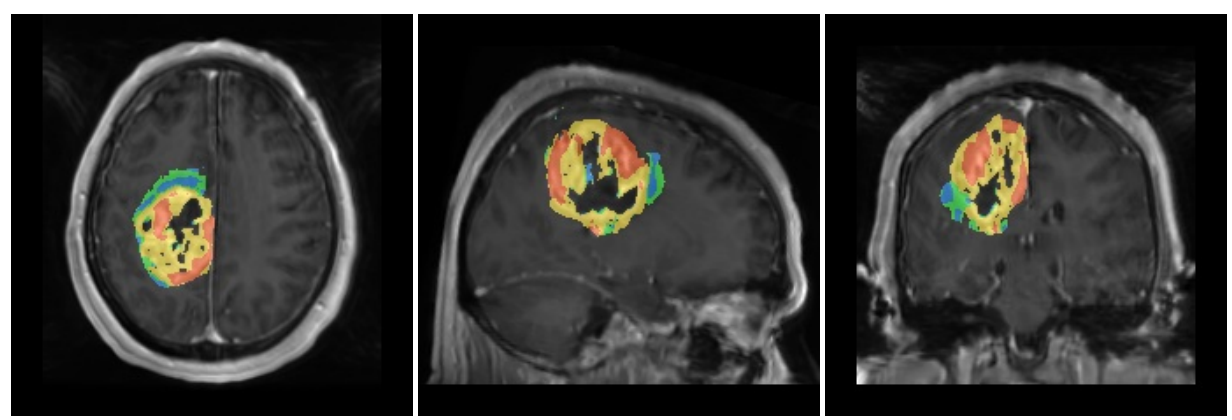

- Hemodynamic DSC tissue signature chart and prototype concentration-time perfusion curves
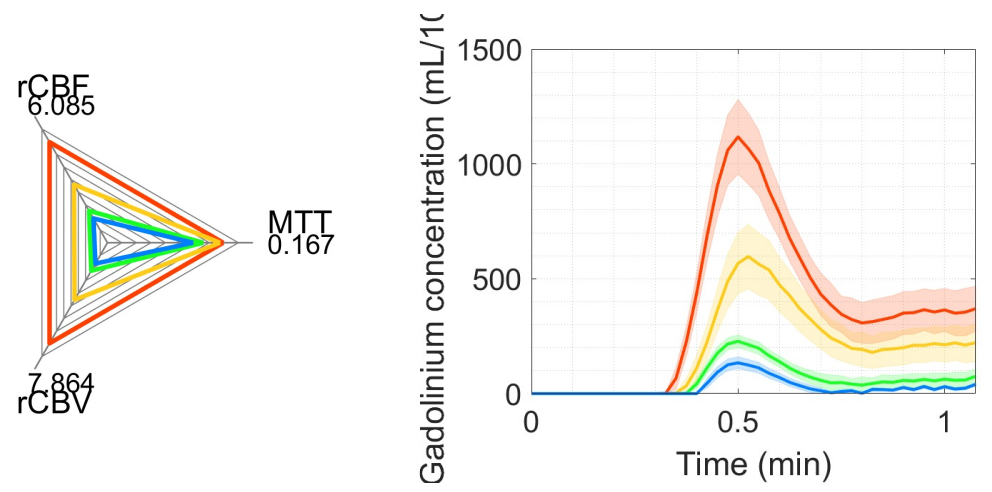

${ }^{1}$ The results contained in this report are only certified for research purposes.

Figure 7.6: Example of the fourth page of a report for a vascular heterogeneity assessment analysis of a glioblastoma. 


\section{DISCLAIMER}

All calculations, measurements and images provided by this software are intended only for scientific research. Any other use is entirely at the discretion and risk of the user. If you do use this software for scientific research please give appropriate credit in publications. The results of the HTS may not be commercially used in any other way without prior approval of the author.

\section{DEFINITION OF THE SERVICES}

Pre-Processing: Pre-processing module attempts to enhance and correct the MR images for its posterior analysis. Several common artefacts are corrected in this module such as magnetic bias field inhomogeneities, noise or spike artefacts. Additionally, automated registration and skull-stripping is conducted to generate a consistent multi-parametric high quality imaging space of the brain.

Segmentation: The anatomical analysis of the glioblastoma requires the delineation of the tumor tissues, which encompass the enhancing tumor, the edema and the necrotic tissues. In this module, we implemented a deep learning approach to provides such tissue identification. Convolutional Neural Networks are employed in combination with a morphological component analysis to identify the pathological structures.

DSC perfusion quantification: DSC quantification involves the computation of the hemodynamic indices obtained from a kinetic analysis of the first pass of a intravenously injected paramagnetic contrast agent. T1 and T2 leakge effects are also corrected to not miss-estimate the hemodynamic biomarkers. The quantified maps computed by this module are: relative Cerebral Blood Volume (rCBV), relative Cerebral Blood Flow (rCBF), Mean Transit Time (MTT) and K2 maps.

Hemodynamic Tissue Signature (HTS): HTS consist on an automated unsupervised method able to describe the vascular heterogeneity of the enhancing tumour and edema tissues in terms of the angiogenic process located at these regions. The HTS provides a characterization of the GBM, whose output is a nosologic map of the tumoral tissues grouped in different vascular sub-comparmtents with their associated MRI fingerprint. We consider 4 vascular sub-compartments for the GBM: the high angiogenic enhancing tumour region (HAT), the low angiogenic enhancing tumour region (LAT), the potentially tumour infilatrated peripheral edema (IPE) and the pure vasogenic edema (VPE). The HTS is able to capture the local heterogeneity of the tumour, hence providing relevant information about its behaviour.
rCBV relative Cerebral Blood Volume
rCBF relative Cerebral Blood Flow
MTT Mean Transit Time
DSC Dynamic Susceptibility Contrast
HTS Hemodynamic Tissue Signature

Figure 7.7: Example of the fifth page of a report for a vascular heterogeneity assessment analysis of a glioblastoma. 
Chapter 7. ONCOhabitats: A system for glioblastoma heterogeneity assessment through Magnetic Resonance Imaging

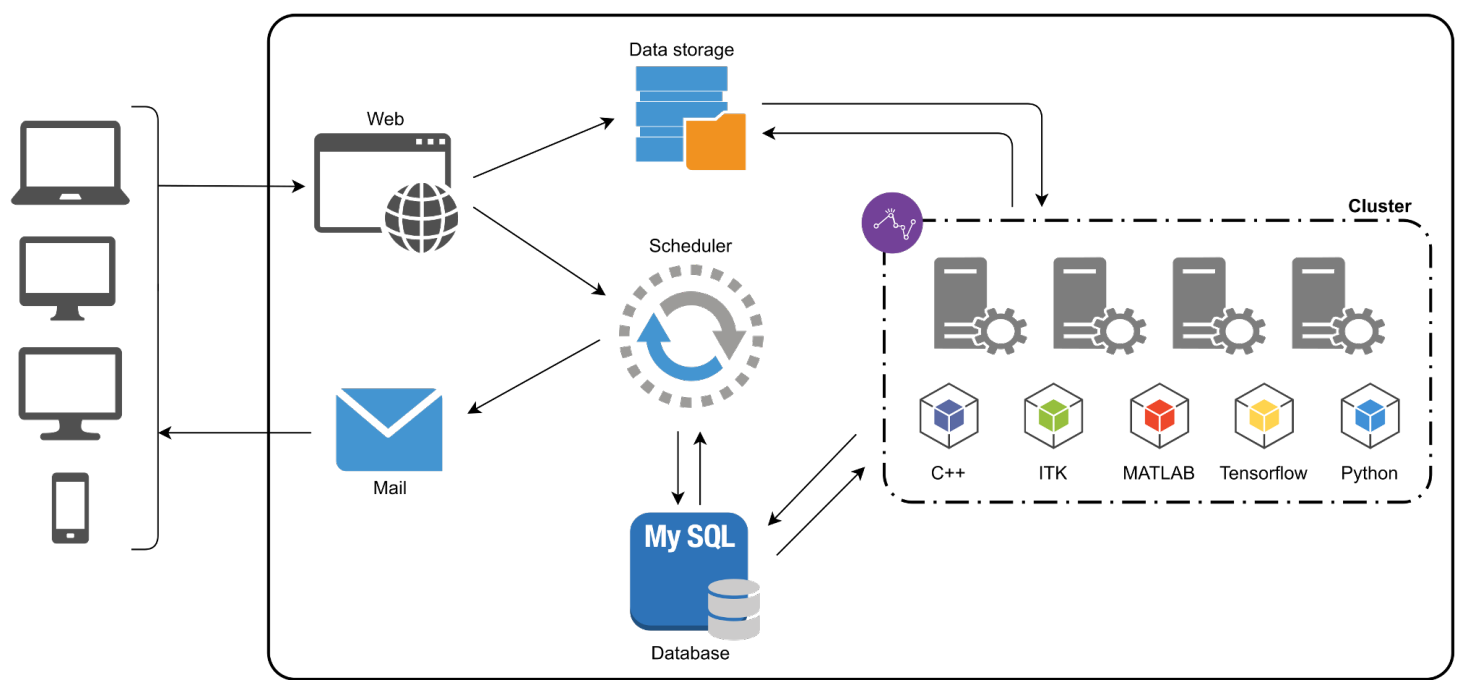

Figure 7.8: ONCOhabitats system architecture.

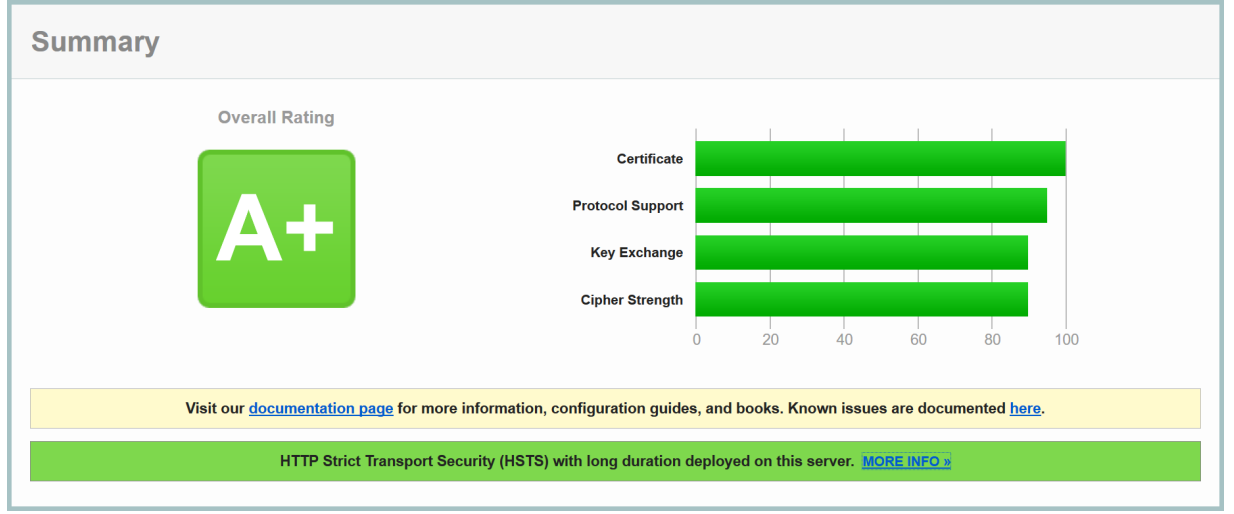

Figure 7.9: ONCOhabitats system security rating evaluated by SSL Labs by Qualy.

libraries. MATLAB ${ }^{\odot}$ and PHP are also employed as scripting languages for different tasks. Tensorflow ${ }^{\text {TM }}$ is used to develop the deep learning segmentation models.

ONCOhabitats has been designed to deal with up to 14 concurrent jobs, each job taking approximately one hour, which yields a theoretical limit of 336 processed cases per day. The terms of use of the system are available in the landing web page, paying particular attention to the GDPR compliance and the non-commercial research purpose of the system.

\section{$7.4 \quad$ Results}

Since each ONCOhabitats service performs a different task, they have been evaluated separately with different datasets and methodologies. The results of the evaluations are presented below. 


\subsubsection{High-grade glioma segmentation service}

High-grade glioma tissue segmentation performance was evaluated according to BRATS evaluation guidelines. Such evaluation comprises the assessment of the segmentation quality of the WT region, the TC region and the ET area. Dice metric, as well as sensitivity and specificity of each region was computed to compare ONCOhabitats results with several state-of-the-art methods.

Table 7.2: Segmentation results obtained by the ONCOhabitats high-grade glioma segmentation service for the BRATS 2017 validation set. ET: Enhancing Tumor, WT: Whole Tumor, TC: Tumor Core

\begin{tabular}{lccc}
\hline & ET & WT & TC \\
Dice & 0.73 & 0.89 & 0.73 \\
Sensitivity & 0.77 & 0.87 & 0.72 \\
Specificity & 0.99 & 0.99 & 0.99 \\
\hline
\end{tabular}

The results of our high-grade glioma segmentation service are presented in Table 7.2. A comparison between the results obtained by ONCOhabitats system and the ones obtained by each participant of the challenge for the BRATS 2017 validation set are presented in Figure 7.10. As the figure shows, ONCOhabitats offers competitive comparable segmentations with the state-of-the-art algorithms, always achieving results above the median of the participants and in most cases close to the third quartile. Moreover, CNN yield segmentations of high specificity, which is a highly desirable property for a medical tool, ensuring a very low false positive rate.

\subsubsection{Glioblastoma vascular heterogeneity assessment service}

An extensive evaluation of the HTS method at different levels is presented in section 5.4 .

First, a statistical evaluation to assess the degree of similarity among the rCBV and rCBF distributions for the HAT, LAT, IPE and VPE habitats was conducted. Global probabilistic deviation metric (Sáez et al, 2017) was employed as a multi-dimensional extension of Jensen-Shannon divergence to measure distances between distributions. The analysis yielded the following average results for the population: $0.88 \pm 0.03$ for rCBV and $0.86 \pm 0.05$ for $\mathrm{rCBF}$. Such results indicated that the perfusion distributions of the habitats for each patient of the study were statistically significantly separated, hence corroborating that the HTS habitats describe regions within the glioblastoma with different hemodynamic behavior.

Second, the prognostic capabilities of the HTS habitats were studied. Cox proportional hazard regression analysis and a Kaplan-Meier study were conducted to measure the degree of correlation of the HTS habitats with patient OS. The maximum rCBV and $\mathrm{rCBF}$ value at each habitat (computed as the $95 \%$ percentile of the distribution) was the marker with better results in concordance with previous studies in the literature (Wetzel et al, 2002). HAT and LAT habitats, as well as IPE yielded positive 
Chapter 7. ONCOhabitats: A system for glioblastoma heterogeneity assessment through Magnetic Resonance Imaging

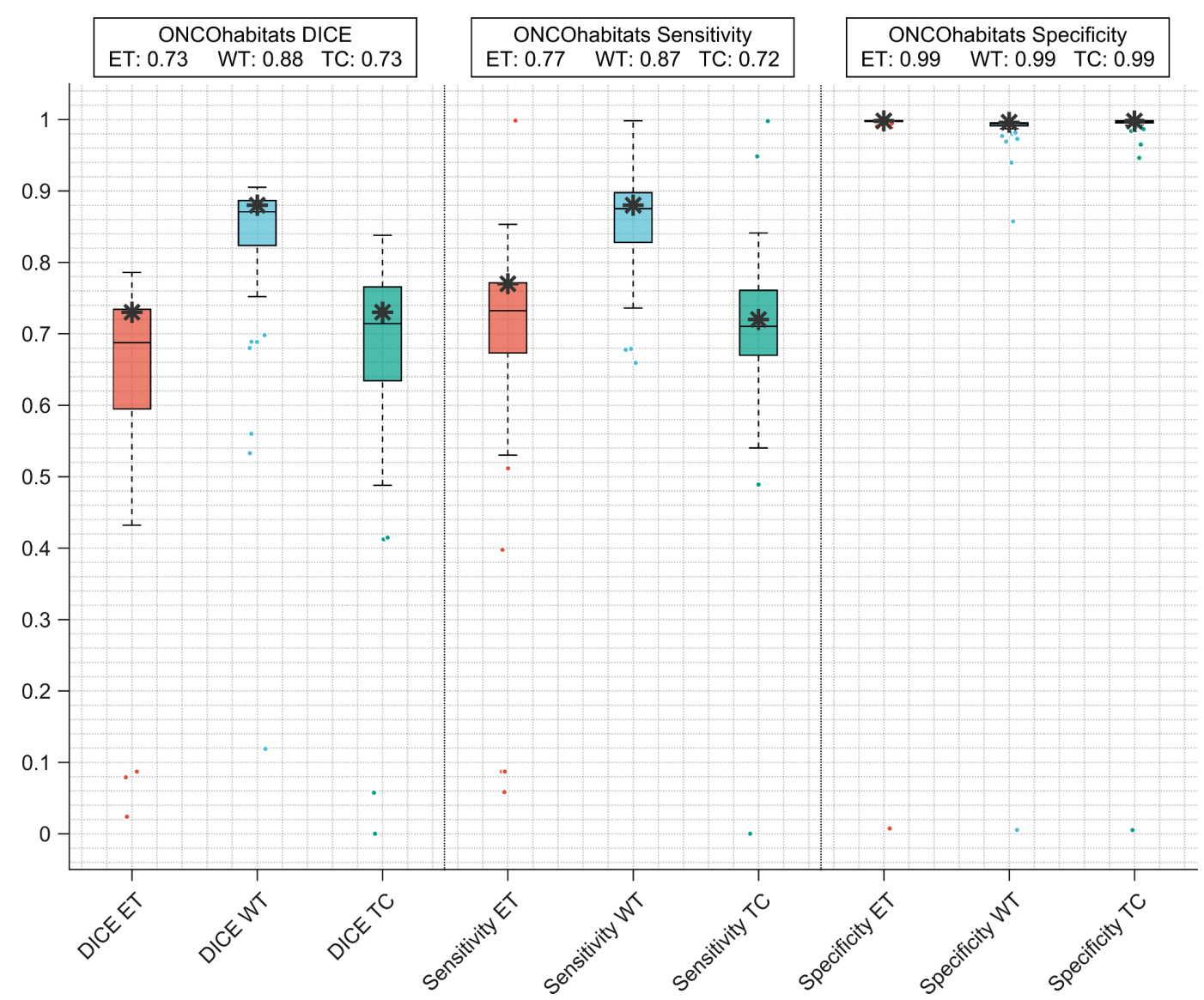

Figure 7.10: Distributions of Dice scores, sensitivities and specificities for the BRATS 2017 validation set results obtained by the participants of the challenge. ONCOhabitats results are indicated with a * marker. ET: Enhancing Tumor, WT: Whole Tumor, TC: Tumor Core

correlations with overall survival for both Cox and Kaplan-Meier studies. BenjaminiHochberg false discovery rate at $\alpha$ level of 0.05 was employed to correct for multiple hypothesis testing, increasing confidence of the statistical results.

\subsection{Discussion}

In this work we presented ONCOhabitats: an on-line open-access system to study different aspects of glioblastoma such as the tumor morphology and the vascular tumor heterogeneity patterns. ONCOhabitats provides the user with consolidated state-ofthe-art techniques based on both deep learning and unsupervised structured learning algorithms previously published in the literature. The system also generates automated radiological reports summarizing the findings of the analysis, in a formal document easily integrable in clinical routine.

The ONCOhabitats high-grade glioma segmentation was compared against the current state-of-the-art methods presented at BRATS 2017 challenge. Our system yields comparable results with these approaches, demonstrating competitive comparable results with no significant differences between them. Our method is completely deterministic and reproducible, which is a highly desirable property to conduct large population 
studies or clinical trials. The higher reproducibility of a system, the greater the likelihood to detect changes in the disease. Additionally, ONCOhabitats implements the Hemodynamic Tissue Signature method presented in (Juan-Albarracín et al, 2018), to study the vascular heterogeneity of the tumor. Glioblastoma vascular heterogeneity has been demonstrated to be a key hallmark to understand the behavior of this masses. The HTS analysis allows to study the different vascular patterns of the lesion, detecting functional habitats within the tissues that have been demonstrated to contain relevant information about patient's survival at a very early stage of the disease.

ONCOhabitats offers these analyses by means of a free web-based solution. We give access to the scientific community not only to our software services but also to our computational resources, avoiding the requirement for medical imaging experts, expensive computational labs and arduous learning curves to develop the technology. We provide a system capable to process about 300 cases per day including MRI preprocessing and standardization, tissue segmentation, DSC perfusion quantification and vascular heterogeneity assessment of the lesion. The system is designed to be immediately scalable by adding new computing machines and also cloud-based services as the workload increases.

The major limitation of our system is the time to process a case. Glioblastoma study involves the analysis of a considerable amount of MRI to capture all the information contained in the lesion, requiring to process a huge amount of data. Currently, MRI preprocessing accounts for the most part of the processing time, so we are currently developing new approaches to optimize this module.

Currently, ONCOhabitats system is participating in the international clinical trial NCT03439332, aimed to validate the ONCOhabitats technology in a multi-centre observational study with 300 patients from hospitals from Spain, Italy, Belgium and Norway. In future work, we plan to extend ONCOhabitats system to handle DWI and Diffusion Tensor Imaging (DTI) sequences, as well as post-surgery and follow-up studies for a longitudinal assessment of the glioblastoma. 
Chapter 7. ONCOhabitats: A system for glioblastoma heterogeneity assessment through Magnetic Resonance Imaging 


\section{Chapter 8}

\section{Concluding remarks and recommendations}

This chapter finalizes the work conducted in this thesis and summarizes the main concluding remarks and recommendations derived from it. Additionally, in this chapter we provide the guidelines for continuing the scientific research and development based on this work.

\subsection{Concluding remarks}

AI medical image analysis is a cornerstone in the future of modern precision medicine. The ability to non-invasively measure morphological and quantitative characteristics of complicated diseases such as glioblastoma is an invaluable aid in successfully combating these lethal lesions. In the particular case of glioblastoma, to date, this tumor still remains a major challenge, as there is no satisfactory therapy for it. Understanding its high heterogeneity, and in particular its vascular heterogeneity, constitutes a key element in advancing the design of effective therapies. Therefore, it is essential to continue the study and research of this neoplasm from different perspectives: its pathology, its molecular and genetic processes, its immunology and, of course, through neuroimaging. The latter is rapidly evolving towards richer and more complex multiparametric acquisitions that require increasingly advanced computational models to harness the raw information they contain. In this sense, this thesis has contributed to the assessment and characterization of the vascular heterogeneity of the glioblastoma by means of unsupervised ML techniques applied to MRI data, able to discover habitats within the lesion with early prognostic capabilities.

This thesis has contributed to the state-of-the-art in the fields of Medical Informatics, Statistics and Probability, Radiology and Nuclear Medicine, Machine Learning and Data Mining and Biomedical Engineering. The scientific publications in top-ranked journals and international conferences derived from this thesis endorse the research carried out in these fields. Furthermore, the methods and technology developed in this thesis have been integrated in a public open-access platform for its use by the medical and research community, or for its posterior industrialization.

The specific concluding remarks of this thesis are listed as follows. 
CR1 Unsupervised learning is confirmed as a viable tool for MRI analysis and pathological pattern detection. We found that, although supervised learning normally achieves better results in well-known tasks such as image segmentation, unsupervised learning is also able to accurately capture MRI patterns related to morphological and physiological characteristics of the lesion. The study conducted in chapter 3 demonstrated the potential of unsupervised learning approaches, showing a consistent behavior across the different datasets, which is a highly desirable property when dealing with heterogeneous data.

This settled the basis for the subsequent contributions carried out in the thesis, where no ground-truth exist from where to learn supervised methods. The performance obtained in this preliminary study in segmenting well-known tissues based on MR intensity patterns gave us evidences and confidence in the following studies conducted in the thesis.

This concluding remark responds to the research question $R Q 1$, covers the objectives $O 1$ and $\mathrm{O}_{2}$ and was derived from the works in publications P1, P2 and P3.

CR2 The SVFMM is a powerful and robust state-of-the-art framework for unsupervised learning of imaging data. The Bayesian nature of this model provides great flexibility to inject existing knowledge into the learning process, to successfully capture spatial redundancy of the images and introduce local regularization. Moreover, it also provides mechanisms to guide the learning process towards plausible solutions aligned with task-specific constraints.

The SVFMM has been the basis for the HTS method presented in this thesis to describe the vascular heterogeneity of glioblastomas. Particularly, we have proposed a variant of the SVFMM combined with the probabilistic NLM scheme that achieves better results compared to the alternative approaches in their family. The proposed approach also simplifies the previous models since the probabilistic NLM weighting function does not introduce additional parameters to the model.

This concluding remark responds to the research question RQ2, covers the objective $O 3$ and was derived from the work in publication P3.

CR3 Early-stage vascular heterogeneity provide crucial information about expected survival of patients with glioblastoma undergoing standard-of-care treatment. The HTS habitats successfully capture this heterogeneity by analyzing the perfusion patterns within the lesion, showing improved association with OS with respect to alternative approaches. Consistently with the literature, habitats related to the enhancing tumor presented strong correlation with patient OS. However, the most important finding is the positive association of the $r C B V_{\max }$ in the IPE habitat with OS. The infiltrated edema today catches all the attention since it is identified as the critical region for many decisive treatments such as surgical resection or radiotherapy, thus increasing the importance of this finding.

Moreover, the HTS relies on a conceptual framework to describe the heterogeneity of a lesion by means of detecting habitats with differentiated MRI functional profiles. This enables a new perspective in the characterization of complex lesions 
under the medical imaging paradigm. This approach introduces a concept-shift in the segmentation of lesions towards the delineation of regions sharing a similar physiological behavior rather than a common morphological appearance.

This concluding remark responds to the research questions $R Q 3$ and $R Q 4$, covers the objectives $\mathrm{O}_{4}$ and $\mathrm{O} 5$ and was derived from the works in publications P5, P6 and $P$ r.

CR4 ONCOhabitats (https://www.oncohabitats.upv.es) platform encapsulates all the original methods and algorithms developed in this thesis, and several state-ofthe-art algorithms for medical image analysis, in a public open-access system free for the medical and research community. ONCOhabitats is a reliable system for the study of glioblastoma that provides an end-to-end analysis of the lesion, from the preprocessing of the raw MRI obtained from the scanner to the final measurement of volumetries and quantitative biomarkers of habitats representing regions with a specific physiological behavior.

ONCOhabitats is designed modularly to allow an easy reuse of the technology, a sustainable development cycle and a high scalability. It is mainly written using open-access state-of-the-art libraries with the aim to positioning the system as a reference platform for the analysis of brain tumors through medical imaging. In this sense, ONCOhabitats not only offers cutting edge technology, but also provides access to their computational resources, allowing a case-analysis-rate of about 300 cases per day.

The software is registered in the technological offer of the UPV and is protected under the patents ES201431289A in Spain, EP3190542A1 in Europe and US20170287133A1 in United States.

This concluding remark responds to the research question $R Q 5$, covers the objective $O 6$ and was derived from the works in publications P4, P8 and P9.

\subsection{Recommendations}

Glioblastoma tumor still remains a lethal disease that requires a tireless multi-disciplinary effort to understand its behavior, evolution, proliferation and survival mechanisms that grant its uncontrollable aggressiveness. Under this scenario, the future of the analysis of such lethal diseases lies in the inclusion of ML techniques, capable of analyzing the vast amount of complex multi-disciplinary medical information available to a patient, with the aim of developing personalized therapies that exploit the particularities of each individual.

The developed methods and research findings performed in this thesis points to the aforementioned direction and can serve as a starting point for further research. In this sense, the following recommendations are suggested.

R1 Despite the unquestionable power, utility and performance that supervised learning is demonstrating nowadays, its ability to discover new knowledge from biomedical data is severely limited by its learning mechanism. This task is, by the opposite, perfectly suited for unsupervised learning. The exploratory nature inherent 
in unsupervised learning provides it with the ability to detect hidden patterns within the data, often imperceptible to the human.

In this regard, we encourage the use of unsupervised learning for medical image analysis and biomedical data mining in general, to build descriptive models of the data capable of capturing subtle patterns that lead to undetectable important findings for the human being. Therefore, unsupervised learning must assume a relevant role in modern medicine to situate ML as an essential tool for the future of personalized therapies.

R2 Learning from structured data, such as MR images, requires ML models capable to exploit the conditional dependencies and spatial correlations associated to these data. Historically, MRFs have proven to be powerful mathematical models to capture the local dependencies encoded in the images. Specifically, SVFMMs constitute a versatile statistical framework to describe heterogeneous structured imaging data under a strong mathematical foundation. On the other hand, the NLM image processing scheme has also proven to achieve state-of-the-art results in many image-related tasks such as denoising, super-resolution, in-painting or patch-based segmentation. We encourage the use of the NLSVFMM algorithm, as it brings together the potential of both approaches in a fully Bayesian statistical model that has demonstrated comparable state-of-the-art performance in a model mathematically less complex than those of its family.

Nevertheless, the current trend in ML focuses on the use of CNNs, since they have demonstrated to be the state-of-the-art models for most of image analysis related tasks. CNNs, however, are mathematical models mainly oriented to the supervised learning paradigm. Therefore, we advocate the need to investigate new architectures and learning schemes to exploit the potential of CNNs in an unsupervised learning scheme. Nowadays, Convolutional AutoEnconders (CAEs) with latent space clustering losses are being raised as the most powerful alternatives for performing unsupervised learning segmentation in images based on CNNs.

R3 The HTS method settles an innovative approach to characterize the vascular heterogeneity of glioblastomas by means of detecting functional habitats within the lesion with differentiated MRI profile. We consider that this conceptual framework provides a powerful tool to explore the internal behavior of a tumor in an objective non-biased data-driven manner. The underlying unsupervised ML approach behind the method allows the heterogeneity of the lesion to be easily explored from different points of view, ranging from varying the number of habitats to find within the lesion, to adding additional MRI sequences, such DWI or NMR relaxometry maps, to enrich the imaging profile of the tumor.

R4 Enhancing tumor vascularity has historically demonstrated strong association with OS of patients affected by glioblastoma. However, there is a lack of consensus in the literature on the $r C B V_{\max }$ quantities that correlate with this outcome. This is probably due to the large variability in the perfusion MRI protocols and 
quantification methods employed, in addition to the manual ROI selection for tumor measurements and perfusion relative normalization techniques.

In this sense, we encourage the use of the HTS method, as it has proven to be robust to highly variable MRI acquisition protocols and manufacturers from different international centers, yielding $r C B V_{\max }$ distributions for the different HTS habitats with no statistically significant differences among most of the centers. This is a highly desirable property to conduct large cross-sectional population studies, where the conclusions of the experiments must be extrapolable to the entire population.

R5 Diffuse infiltration is one of the most crucial aspects of the glioblastoma, as it renders total resection impossible and, therefore, progression after surgery is almost inevitable. Thus, detecting the areas of potentially infiltrated tumor cells is of clinical significance for many targeted interventions such as surgery or radiotherapy. The findings of this thesis related to the IPE habitat strongly positions the HTS method as a primary tool to study the morphology, distribution, profile and characteristics of the infiltration region. The positive association of the IPE habitat with patient OS confirms the importance of this habitat and enhances the HTS as a method to study the heterogeneity of the glioblastoma.

R6 Glioblastoma heterogeneity is evidenced at multiple levels, ranging from macroscopic co-existence of malignant tissues, to genetic alterations that derive in different glioblastoma molecular sub-types and to longitudinal evolution of mutant glioblastomas. Molecular analysis is nowadays crucial to investigate the response of the tumor to different therapies and its mechanisms of proliferation. Nevertheless, multi-parametric medical image analysis of the tumor has gained a lot of attention in the past years since it is demonstrating strong associations with relevant clinical outcomes such as OS, tumor grading or genetic mutations.

In this sense, we recommend that further research on glioblastoma using medical imaging takes into account molecular and genetic alterations to enhance and complement the imaging information, with the goal of designing more accurate models capable of predicting clinical outcomes more reliably.

R7 Multi-disciplinary cross-sectional research is nowadays necessary to find effective therapies for such a lethal disease as the glioblastoma. From the technological point of view, it is almost impossible for each research group to develop from scratch all the necessary state-of-the-art technology needed to analyze the different sources of information available for glioblastoma, i.e. MRI, genetic profiling, monitoring events, electronic health records, etc.

In this regard, we encourage research groups specialized in particular topics must make an effort to provide open access to their methods to facilitate the crosssectional research between different disciplines, since it will accelerate and improve the research on these complex and lethal diseases. 
Chapter 8. Concluding remarks and recommendations 


\section{Bibliography}

Abramson RG, Burton KR, Yu JPJ, Scalzetti EM, Yankeelov TE, Rosenkrantz AB, Mendiratta-Lala M, Bartholmai BJ, Ganeshan D, Lenchik L, Subramaniam RM (2015) Methods and challenges in quantitative imaging biomarker development. Academic Radiology 22(1):25-32, DOI 10.1016/j. acra.2014.09.001

Ahmed S, Iftekharuddin KM, Vossough A (2011) Efficacy of Texture, Shape, and Intensity Feature Fusion for Posterior-Fossa Tumor Segmentation in MRI. IEEE Transactions on Information Technology in Biomedicine 15(2):206-213, DOI 10.1109/TITB.2011.2104376

Akagi Y, Yoshimoto K, Hata N, Kuga D, Hatae R, Amemiya T, Sangatsuda Y, Suzuki SO, Iwaki T, Mizoguchi M, Iihara K (2018) Reclassification of 400 consecutive glioma cases based on the revised 2016who classification. Brain Tumor Pathology 35(2):81-89, DOI 10.1007/s10014-018-0313-4

Akbari H, Macyszyn L, Da X, Wolf RL, Bilello M, Verma R, O'Rourke DM, Davatzikos C (2014) Pattern analysis of dynamic susceptibility contrast-enhanced MR imaging demonstrates peritumoral tissue heterogeneity. Radiology 273(2):502-510, DOI 10.1148/radiol.14132458

Alves TR, Lima FRS, Kahn SA, Lobo D, Dubois LGF, Soletti R, Borges H, Neto VM (2011) Glioblastoma cells: a heterogeneous and fatal tumor interacting with the parenchyma. Life Sciences 89(1516):532-539, DOI 10.1016/j.lfs.2011.04.022

Andersen IK, Szymkowiak A, Rasmussen CE, Hanson LG, Marstrand JR, Larsson HBW, Hansen LK (2002) Perfusion quantification using Gaussian process deconvolution. Magnetic Resonance in Medicine 48(2):351-361, DOI 10.1002/mrm.10213

Arthur D, Vassilvitskii S (2007) K-means++: The Advantages of Careful Seeding. In: Proceedings of the Eighteenth Annual ACM-SIAM Symposium on Discrete Algorithms, Society for Industrial and Applied Mathematics, Philadelphia, PA, USA, SODA '07, pp 1027-1035, event-place: New Orleans, Louisiana

Artzi M, Bokstein F, Blumenthal DT, Aizenstein O, Liberman G, Corn BW, Ben Bashat D (2014) Differentiation between vasogenic-edema versus tumor-infiltrative area in patients with glioblastoma during bevacizumab therapy: a longitudinal MRI study. European Journal of Radiology 83(7):1250-1256, DOI 10.1016/j.ejrad.2014.03.026

Avants BB, Epstein CL, Grossman M, Gee JC (2008) Symmetric diffeomorphic image registration with cross-correlation: evaluating automated labeling of elderly and neurodegenerative brain. Medical Image Analysis 12(1):26-41, DOI 10.1016/j.media.2007.06.004

Avants BB, Tustison NJ, Wu J, Cook PA, Gee JC (2011) An open source multivariate framework for n-tissue segmentation with evaluation on public data. Neuroinformatics 9(4):381-400, DOI 10.1007/s12021-011-9109-y

Avants BB, Tustison NJ, Stauffer M, Song G, Wu B, Gee JC (2014) The Insight ToolKit image registration framework. Frontiers in Neuroinformatics 8:44, DOI 10.3389/fninf.2014.00044 
Azuaje F (2019) Artificial intelligence for precision oncology: beyond patient stratification. npj Precision Oncology 3(1):1-5, DOI 10.1038/s41698-019-0078-1

Bae S, Choi YS, Ahn SS, Chang JH, Kang SG, Kim EH, Kim SH, Lee SK (2018) Radiomic MRI Phenotyping of Glioblastoma: Improving Survival Prediction. Radiology 289(3):797-806, DOI 10.1148/radiol.2018180200

Barajas RF, Chang JS, Sneed PK, Segal MR, McDermott MW, Cha S (2009) Distinguishing recurrent intra-axial metastatic tumor from radiation necrosis following gamma knife radiosurgery using dynamic susceptibility-weighted contrast-enhanced perfusion MR imaging. AJNR American journal of neuroradiology 30(2):367-372, DOI 10.3174/ajnr.A1362

Bauer S, Nolte LP, Reyes M (2011) Fully automatic segmentation of brain tumor images using support vector machine classification in combination with hierarchical conditional random field regularization. Medical image computing and computer-assisted intervention: MICCAI International Conference on Medical Image Computing and Computer-Assisted Intervention 14(Pt 3):354-361, DOI 10.1007/978-3-642-23626-6\_44

Bauer S, Wiest R, Nolte LP, Reyes M (2013) A survey of MRI-based medical image analysis for brain tumor studies. Physics in Medicine and Biology 58(13):R97-129, DOI 10.1088/0031-9155/58/13/ R97

Bengio Y (2017) Deep Learning. MIT Press, Cambridge, Massachusetts

Benjamini Y, Hochberg Y (1995) Controlling the False Discovery Rate: A Practical and Powerful Approach to Multiple Testing. Journal of the Royal Statistical Society Series B (Methodological) $57(1): 289-300$

Bezdek JC (1981) Pattern Recognition with Fuzzy Objective Function Algorithms. Advanced Applications in Pattern Recognition, Springer US

Birchfield S, Tomasi C (1998) Depth discontinuities by pixel-to-pixel stereo. In: Sixth International Conference on Computer Vision (IEEE Cat. No.98CH36271), pp 1073-1080, DOI 10.1109/ICCV. 1998.710850

Bishop C (2006) Pattern Recognition and Machine Learning. Information Science and Statistics, Springer-Verlag, New York

Bishop CM (1995) Neural Networks for Pattern Recognition. Oxford University Press, Inc., New York, NY, USA

Blake A, Rother C (2011) Markov random fields for vision and image processing. MIT Press, Cambridge, Mass.

Blekas K, Likas A, Galatsanos NP, Lagaris IE (2005) A spatially constrained mixture model for image segmentation. IEEE Transactions on Neural Networks 16(2):494-498, DOI 10.1109/TNN. 2004.841773

Boxerman JL, Schmainda KM, Weisskoff RM (2006) Relative cerebral blood volume maps corrected for contrast agent extravasation significantly correlate with glioma tumor grade, whereas uncorrected maps do not. AJNR American journal of neuroradiology 27(4):859-867

Boykov Y, Veksler O, Zabih R (2001) Fast approximate energy minimization via graph cuts. IEEE Transactions on Pattern Analysis and Machine Intelligence 23(11):1222-1239, DOI 10.1109/34. 969114 
Buades A, Coll B, Morel J (2005a) A non-local algorithm for image denoising. In: 2005 IEEE Computer Society Conference on Computer Vision and Pattern Recognition (CVPR'05), vol 2, pp 60-65 vol. 2, DOI 10.1109/CVPR.2005.38

Buades A, Coll B, Morel J (2005b) A Review of Image Denoising Algorithms, with a New One. Multiscale Modeling \& Simulation 4(2):490-530, DOI 10.1137/040616024

Calamante F, Gadian DG, Connelly A (2003) Quantification of bolus-tracking MRI: Improved characterization of the tissue residue function using Tikhonov regularization. Magnetic Resonance in Medicine 50(6):1237-1247, DOI 10.1002/mrm.10643

Chang YCC, Ackerstaff E, Tschudi Y, Jimenez B, Foltz W, Fisher C, Lilge L, Cho H, Carlin S, Gillies RJ, Balagurunathan Y, Yechieli RL, Subhawong T, Turkbey B, Pollack A, Stoyanova R (2017) Delineation of Tumor Habitats based on Dynamic Contrast Enhanced MRI. Scientific Reports 7(1):9746, DOI 10.1038/s41598-017-09932-5

Chen JJ, Smith MR, Frayne R (2005) Advantages of frequency-domain modeling in dynamicsusceptibility contrast magnetic resonance cerebral blood flow quantification. Magnetic Resonance in Medicine 53(3):700-707, DOI 10.1002/mrm.20382

Cheng Y (1995) Mean shift, mode seeking, and clustering. IEEE Transactions on Pattern Analysis and Machine Intelligence 17(8):790-799, DOI 10.1109/34.400568

Connelly A, Calamante F, Willats L (2006) Improved deconvolution of bolus tracking data using wavelet thresholding. Proc 14th Sci Meeting Int Soc Magn Reson Med pp 3563-3563

Crimi A, Menze B, Maier O, Reyes M, Handels H (eds) (2015) Brainlesion: Glioma, Multiple Sclerosis, Stroke and Traumatic Brain Injuries: First International Workshop, Brainles 2015, Held in Conjunction with MICCAI 2015, Munich, Germany, October 5, 2015, Revised Selected Papers. Image Processing, Computer Vision, Pattern Recognition, and Graphics, Springer International Publishing

Crimi A, Menze B, Maier O, Reyes M, Winzeck S, Handels H (eds) (2016) Brainlesion: Glioma, Multiple Sclerosis, Stroke and Traumatic Brain Injuries: Second International Workshop, BrainLes 2016, with the Challenges on BRATS, ISLES and mTOP 2016, Held in Conjunction with MICCAI 2016, Athens, Greece, October 17, 2016, Revised Selected Papers. Image Processing, Computer Vision, Pattern Recognition, and Graphics, Springer International Publishing

Crimi A, Bakas S, Kuijf HJ, Menze B, Reyes M (eds) (2017) Brainlesion: Glioma, Multiple Sclerosis, Stroke and Traumatic Brain Injuries: Third International Workshop, BrainLes 2017, Held in Conjunction with MICCAI 2017, Quebec City, QC, Canada, September 14, 2017, Revised Selected Papers. Image Processing, Computer Vision, Pattern Recognition, and Graphics, Springer International Publishing

Crimi A, Bakas S, Kuijf HJ, Keyvan F, Reyes M, Walsum Tv (eds) (2018) Brainlesion: Glioma, Multiple Sclerosis, Stroke and Traumatic Brain Injuries: 4th International Workshop, BrainLes 2018, Held in Conjunction with MICCAI 2018, Granada, Spain, September 16, 2018, Revised Selected Papers, Part II. Image Processing, Computer Vision, Pattern Recognition, and Graphics, Springer International Publishing

Cui Y, Tha KK, Terasaka S, Yamaguchi S, Wang J, Kudo K, Xing L, Shirato H, Li R (2016) Prognostic Imaging Biomarkers in Glioblastoma: Development and Independent Validation on the Basis of Multiregion and Quantitative Analysis of MR Images. Radiology 278(2):546-553, DOI 10.1148/ radiol.2015150358

Damadian R (1971) Tumor Detection by Nuclear Magnetic Resonance. Science 171(3976):1151-1153, DOI 10.1126/science.171.3976.1151 
Dang L, Jin S, Su SM (2010) IDH mutations in glioma and acute myeloid leukemia. Trends in Molecular Medicine 16(9):387-397, DOI 10.1016/j.molmed.2010.07.002

Das S, Marsden PA (2013) Angiogenesis in Glioblastoma. The New England journal of medicine 369(16):1561-1563, DOI 10.1056/NEJMcibr1309402

De Guio F, Jouvent E, Biessels GJ, Black SE, Brayne C, Chen C, Cordonnier C, De Leeuw FE, Dichgans M, Doubal F, Duering M, Dufouil C, Duzel E, Fazekas F, Hachinski V, Ikram MA, Linn J, Matthews PM, Mazoyer B, Mok V, Norrving B, O’Brien JT, Pantoni L, Ropele S, Sachdev P, Schmidt R, Seshadri S, Smith EE, Sposato LA, Stephan B, Swartz RH, Tzourio C, van Buchem M, van der Lugt A, van Oostenbrugge R, Vernooij MW, Viswanathan A, Werring D, Wollenweber F, Wardlaw JM, Chabriat H (2016) Reproducibility and variability of quantitative magnetic resonance imaging markers in cerebral small vessel disease. Journal of Cerebral Blood Flow and Metabolism: Official Journal of the International Society of Cerebral Blood Flow and Metabolism 36(8):1319 1337, DOI 10.1177/0271678X16647396

De Palma M, Biziato D, Petrova TV (2017) Microenvironmental regulation of tumour angiogenesis. Nature Reviews Cancer 17(8):457-474, DOI 10.1038/nrc.2017.51

Deimling Av (ed) (2009) Gliomas. Recent Results in Cancer Research, Springer-Verlag, Berlin Heidelberg

Demerath T, Simon-Gabriel CP, Kellner E, Schwarzwald R, Lange T, Heiland DH, Reinacher P, Staszewski O, Mast H, Kiselev VG, Egger K, Urbach H, Weyerbrock A, Mader I (2017) Mesoscopic imaging of glioblastomas: Are diffusion, perfusion and spectroscopic measures influenced by the radiogenetic phenotype? The Neuroradiology Journal 30(1):36-47, DOI 10.1177/1971400916678225

Dempster AP, Laird NM, Rubin DB (1977) Maximum Likelihood from Incomplete Data via the EM Algorithm. Journal of the Royal Statistical Society Series B (Methodological) 39(1):1-38

Deoni SCL (2010) Quantitative relaxometry of the brain. Topics in magnetic resonance imaging: TMRI 21(2):101-113, DOI 10.1097/RMR.0b013e31821e56d8

Dextraze K, Saha A, Kim D, Narang S, Lehrer M, Rao A, Narang S, Rao D, Ahmed S, Madhugiri V, Fuller CD, Kim MM, Krishnan S, Rao G, Rao A (2017) Spatial habitats from multiparametric MR imaging are associated with signaling pathway activities and survival in glioblastoma. Oncotarget 8(68):112,992-113,001, DOI 10.18632/oncotarget.22947

Diaz I, Boulanger P, Greiner R, Murtha A (2011) A critical review of the effects of de-noising algorithms on MRI brain tumor segmentation. Conference proceedings: Annual International Conference of the IEEE Engineering in Medicine and Biology Society IEEE Engineering in Medicine and Biology Society Annual Conference 2011:3934-3937, DOI 10.1109/IEMBS.2011.6090977

Dolecek TA, Propp JM, Stroup NE, Kruchko C (2012) CBTRUS statistical report: primary brain and central nervous system tumors diagnosed in the United States in 2005-2009. Neuro-Oncology 14 Suppl 5:v1-49, DOI 10.1093/neuonc/nos218

Donahue KM, Krouwer HG, Rand SD, Pathak AP, Marszalkowski CS, Censky SC, Prost RW (2000) Utility of simultaneously acquired gradient-echo and spin-echo cerebral blood volume and morphology maps in brain tumor patients. Magnetic Resonance in Medicine 43(6):845-853, DOI 10.1002/1522-2594(200006)43:6〈845::aid-mrm10〉3.0.co;2-j

Dong H, Yang G, Liu F, Mo Y, Guo Y (2017) Automatic Brain Tumor Detection and Segmentation Using U-Net Based Fully Convolutional Networks. In: Valdés Hernández M, González-Castro V (eds) Medical Image Understanding and Analysis, Springer International Publishing, Communications in Computer and Information Science, pp 506-517 
Doyle S, Vasseur F, Dojat M, Forbes F (2013) Fully automatic brain tumor segmentation from multiple MR sequences using hidden Markov fields and variational EM. Proceedings of NCI-MICCAI BRATS 2013 1(1):18-22

Drabycz SA, Brown RA, Law AG, Mitchell JR (2005) Maximum entropy deconvolution for dynamic susceptibility contrast magnetic resonance imaging. In: Proceedings of the 5th IASTED International Conference on Visualization, Imaging, and Image Processing, VIIP 2005, pp 442-447

Duda RO, Hart PE, Stork DG (2000) Pattern Classification (2Nd Edition). Wiley-Interscience, New York, NY, USA

Dunn JC (1973) A Fuzzy Relative of the ISODATA Process and Its Use in Detecting Compact WellSeparated Clusters. Journal of Cybernetics 3(3):32-57, DOI 10.1080/01969727308546046

Elmghirbi R, Nagaraja TN, Brown SL, Panda S, Aryal MP, Keenan KA, Bagher-Ebadian H, Cabral G, Ewing JR (2017) Acute Temporal Changes of MRI-Tracked Tumor Vascular Parameters after Combined Anti-angiogenic and Radiation Treatments in a Rat Glioma Model: Identifying Signatures of Synergism. Radiation Research 187(1):79-88, DOI 10.1667/RR14358.1

Emblem KE, Nedregaard B, Nome T, Due-Tonnessen P, Hald JK, Scheie D, Borota OC, Cvancarova M, Bjornerud A (2008) Glioma grading by using histogram analysis of blood volume heterogeneity from MR-derived cerebral blood volume maps. Radiology 247(3):808-817, DOI 10.1148/radiol. 2473070571

Ester M, Kriegel HP, Sander J, Xu X (1996) A Density-based Algorithm for Discovering Clusters a Density-based Algorithm for Discovering Clusters in Large Spatial Databases with Noise. In: Proceedings of the Second International Conference on Knowledge Discovery and Data Mining, AAAI Press, Portland, Oregon, KDD’96, pp 226-231

Festa J, Pereira S, Mariz J, Sousa N (2013) Automatic Brain Tumor Segmentation of Multi-Sequence MR Images Using Random Decision Forests. Proceedings of NCI-MICCAI BRATS 2013 1(1):23-26

Fletcher-Heath LM, Hall LO, Goldgof DB, Murtagh FR (2001) Automatic segmentation of nonenhancing brain tumors in magnetic resonance images. Artificial Intelligence in Medicine 21(1$3): 43-63$

Fonov V, Evans A, Mckinstry R, Almli C, Collins L (2009) Unbiased nonlinear average age-appropriate brain templates from birth to adulthood. Neuroimage 47, DOI 10.1016/S1053-8119(09)70884-5

Fonov V, Evans AC, Botteron K, Almli CR, McKinstry RC, Collins DL, Brain Development Cooperative Group (2011) Unbiased average age-appropriate atlases for pediatric studies. NeuroImage 54(1):313-327, DOI 10.1016/j.neuroimage.2010.07.033

Fuster-Garcia E, Juan-Albarracín J, García-Ferrando GA, Martí-Bonmatí L, Aparici-Robles F, García-Gómez JM (2018) Improving the estimation of prognosis for glioblastoma patients by MR based hemodynamic tissue signatures. NMR in biomedicine 31(12):e4006, DOI 10.1002/nbm.4006

Gately L, McLachlan SA, Dowling A, Philip J (2017) Life beyond a diagnosis of glioblastoma: a systematic review of the literature. Journal of Cancer Survivorship: Research and Practice 11(4):447452, DOI 10.1007/s11764-017-0602-7

Geyer CJ, Thompson EA (1992) Constrained Monte Carlo Maximum Likelihood for Dependent Data. Journal of the Royal Statistical Society Series B (Methodological) 54(3):657-699

Gladson CL, Prayson RA, Liu WM (2010) The Pathobiology of Glioma Tumors. Annual review of pathology 5:33-50, DOI 10.1146/annurev-pathol-121808-102109 
Gordillo N, Montseny E, Sobrevilla P (2013) State of the art survey on MRI brain tumor segmentation. Magnetic Resonance Imaging 31(8):1426-1438, DOI 10.1016/j.mri.2013.05.002

Gudbjartsson H, Patz S (1995) The Rician Distribution of Noisy MRI Data. Magnetic resonance in medicine 34(6):910-914

Guo L, Wang G, Feng Y, Yu T, Guo Y, Bai X, Ye Z (2016) Diffusion and perfusion weighted magnetic resonance imaging for tumor volume definition in radiotherapy of brain tumors. Radiation Oncology (London, England) 11(1):123, DOI 10.1186/s13014-016-0702-y

Hammersley JM, Clifford P (1971) Markov fields on finite graphs and lattices

Hardee ME, Zagzag D (2012) Mechanisms of glioma-associated neovascularization. The American Journal of Pathology 181(4):1126-1141, DOI 10.1016/j.ajpath.2012.06.030

He K, Zhang X, Ren S, Sun J (2016) Deep Residual Learning for Image Recognition. In: 2016 IEEE Conference on Computer Vision and Pattern Recognition (CVPR), pp 770-778, DOI 10.1109/ CVPR.2016.90

Hebb DO (2002) The Organization of Behavior: A Neuropsychological Theory, edición: 1 edn. Psychology Press, Mahwah, N.J

Hirai T, Murakami R, Nakamura H, Kitajima M, Fukuoka H, Sasao A, Akter M, Hayashida Y, Toya R, Oya N, Awai K, Iyama K, Kuratsu Ji, Yamashita Y (2008) Prognostic value of perfusion MR imaging of high-grade astrocytomas: long-term follow-up study. AJNR American journal of neuroradiology 29(8):1505-1510, DOI 10.3174/ajnr.A1121

Hu LS, Baxter LC, Smith KA, Feuerstein BG, Karis JP, Eschbacher JM, Coons SW, Nakaji P, Yeh RF, Debbins J, Heiserman JE (2009) Relative cerebral blood volume values to differentiate high-grade glioma recurrence from posttreatment radiation effect: direct correlation between image-guided tissue histopathology and localized dynamic susceptibility-weighted contrast-enhanced perfusion MR imaging measurements. AJNR American journal of neuroradiology 30(3):552-558, DOI 10. 3174/ajnr.A1377

Inda MdM, Bonavia R, Seoane J (2014) Glioblastoma Multiforme: A Look Inside Its Heterogeneous Nature. Cancers 6(1):226-239, DOI 10.3390/cancers6010226

Ivakhnenko AG, Lapa VG (1967) Cybernetics and forecasting techniques. American Elsevier Pub. Co.

Jackson A, O'Connor JPB, Parker GJM, Jayson GC (2007) Imaging tumor vascular heterogeneity and angiogenesis using dynamic contrast-enhanced magnetic resonance imaging. Clinical Cancer Research: An Official Journal of the American Association for Cancer Research 13(12):3449-3459, DOI 10.1158/1078-0432.CCR-07-0238

Jain KK (2018) A Critical Overview of Targeted Therapies for Glioblastoma. Frontiers in Oncology 8, DOI 10.3389/fonc.2018.00419

Jain R, Poisson L, Narang J, Gutman D, Scarpace L, Hwang SN, Holder C, Wintermark M, Colen RR, Kirby J, Freymann J, Brat DJ, Jaffe C, Mikkelsen T (2013) Genomic mapping and survival prediction in glioblastoma: molecular subclassification strengthened by hemodynamic imaging biomarkers. Radiology 267(1):212-220, DOI 10.1148/radiol.12120846

Jain R, Poisson LM, Gutman D, Scarpace L, Hwang SN, Holder CA, Wintermark M, Rao A, Colen RR, Kirby J, Freymann J, Jaffe CC, Mikkelsen T, Flanders A (2014) Outcome prediction in patients with glioblastoma by using imaging, clinical, and genomic biomarkers: focus on the nonenhancing component of the tumor. Radiology 272(2):484-493, DOI 10.1148/radiol.14131691 
Jena A, Taneja S, Gambhir A, Mishra AK, D'souza MM, Verma SM, Hazari PP, Negi P, Jhadav GKR, Sogani SK (2016) Glioma Recurrence Versus Radiation Necrosis: Single-Session Multiparametric Approach Using Simultaneous O-(2-18f-Fluoroethyl)-L-Tyrosine PET/MRI. Clinical Nuclear Medicine 41(5):e228-236, DOI 10.1097/RLU.0000000000001152

Jenkinson M, Beckmann CF, Behrens TEJ, Woolrich MW, Smith SM (2012) FSL. NeuroImage 62(2):782-790, DOI 10.1016/j.neuroimage.2011.09.015

Jensen RL, Mumert ML, Gillespie DL, Kinney AY, Schabel MC, Salzman KL (2014) Preoperative dynamic contrast-enhanced MRI correlates with molecular markers of hypoxia and vascularity in specific areas of intratumoral microenvironment and is predictive of patient outcome. NeuroOncology 16(2):280-291, DOI 10.1093/neuonc/not148

Jensen TR, Schmainda KM (2009) Computer-aided detection of brain tumor invasion using multiparametric MRI. Journal of magnetic resonance imaging: JMRI 30(3):481-489, DOI 10.1002/jmri.21878

Juan-Albarracín J, Fuster-Garcia E, García-Gómez JM (2015a) Hierarchical tissue-guided glioblastoma segmentation based on dca-svfmm. In: II International Symposium on Clinical and Basic Investigation in Glioblastoma, p 101

Juan-Albarracín J, Fuster-Garcia E, Manjón JV, Robles M, Aparici F, Martí-Bonmatí L, GarcíaGómez JM (2015b) Automated Glioblastoma Segmentation Based on a Multiparametric Structured Unsupervised Classification. PLOS ONE 10(5):e0125,143, DOI 10.1371/journal.pone.0125143

Juan-Albarracín J, Fuster-Garcia E, García-Gómez JM (2016) An Online Platform for the Automatic Reporting of Multi-parametric Tissue Signatures: A Case Study in Glioblastoma. In: Crimi A, Menze B, Maier O, Reyes M, Winzeck S, Handels H (eds) Brainlesion: Glioma, Multiple Sclerosis, Stroke and Traumatic Brain Injuries, Springer International Publishing, Lecture Notes in Computer Science, pp 43-51

Juan-Albarracín J, Fuster-Garcia E, García-Gómez JM (2017) MTSimaging: multiparametric image analysis services for vascular characterization of glioblastoma. In: 34th Annual Scientific Meeting in European Society for Magnetic Resonance in Medicine and Biology - ESMRMB 2017, p 738

Juan-Albarracín J, Fuster-Garcia E, Pérez-Girbés A, Aparici-Robles F, Alberich-Bayarri n, RevertVentura A, Martí-Bonmatí L, García-Gómez JM (2018) Glioblastoma: Vascular Habitats Detected at Preoperative Dynamic Susceptibility-weighted Contrast-enhanced Perfusion MR Imaging Predict Survival. Radiology 287(3):944-954, DOI 10.1148/radiol.2017170845

Juan-Albarracín J, Fuster-Garcia E, García-Ferrando GA, García-Gómez JM (2019a) ONCOhabitats: A system for glioblastoma heterogeneity assessment through MRI. International Journal of Medical Informatics 128:53-61, DOI 10.1016/j.ijmedinf.2019.05.002

Juan-Albarracín J, Fuster-Garcia E, García-Gómez JM (2019b) Non-local Spatially Varying Finite Mixture Models for Image Segmentation. Statistics and Computing p Accepted for publication

Juan-Albarracín J, Fuster-Garcia E, Álvarez Torres MdM, Chelebian E, García-Gómez JM (2019c) ONCOhabitats glioma segmentation model. In: Crimi A, Menze B, Maier O, Reyes M, Winzeck S, Handels H (eds) Brainlesion: Glioma, Multiple Sclerosis, Stroke and Traumatic Brain Injuries, Springer International Publishing, Lecture Notes in Computer Science, p In press

Kann BH, Thompson R, Thomas CR, Dicker A, Aneja S (2019) Artificial Intelligence in Oncology: Current Applications and Future Directions. Oncology (Williston Park, NY) 33(2):46-53

Kargiotis O, Rao JS, Kyritsis AP (2006) Mechanisms of angiogenesis in gliomas. Journal of NeuroOncology 78(3):281-293, DOI 10.1007/s11060-005-9097-6 
Kassner A, Thornhill RE (2010) Texture analysis: a review of neurologic MR imaging applications. AJNR American journal of neuroradiology 31(5):809-816, DOI 10.3174/ajnr.A2061

Kelley HJ (1960) Gradient Theory of Optimal Flight Paths. ARS Journal 30(10):947-954, DOI $10.2514 / 8.5282$

Klein A, Andersson J, Ardekani BA, Ashburner J, Avants B, Chiang MC, Christensen GE, Collins DL, Gee J, Hellier P, Song JH, Jenkinson M, Lepage C, Rueckert D, Thompson P, Vercauteren T, Woods RP, Mann JJ, Parsey RV (2009) Evaluation of 14 nonlinear deformation algorithms applied to human brain MRI registration. NeuroImage 46(3):786-802, DOI 10.1016/j.neuroimage.2008.12.037

Knopp EA, Cha S, Johnson G, Mazumdar A, Golfinos JG, Zagzag D, Miller DC, Kelly PJ, Kricheff II (1999) Glial neoplasms: dynamic contrast-enhanced T2*-weighted MR imaging. Radiology 211(3):791-798, DOI 10.1148/radiology.211.3.r99jn46791

Knutsson L, Ståhlberg F, Wirestam R (2010) Absolute quantification of perfusion using dynamic susceptibility contrast MRI: pitfalls and possibilities. Magma (New York, NY) 23(1):1-21, DOI 10.1007/s10334-009-0190-2

Komodakis N, Tziritas G (2007) Approximate Labeling via Graph Cuts Based on Linear Programming. IEEE Transactions on Pattern Analysis and Machine Intelligence 29(8):1436-1453, DOI 10.1109/TPAMI.2007.1061

Komodakis N, Tziritas G, Paragios N (2008) Performance vs Computational Efficiency for Optimizing Single and Dynamic MRFs: Setting the State of the Art with Primal-dual Strategies. Comput Vis Image Underst 112(1):14-29, DOI 10.1016/j.cviu.2008.06.007

Krizhevsky A, Sutskever I, Hinton GE (2012) ImageNet Classification with Deep Convolutional Neural Networks. In: Proceedings of the 25th International Conference on Neural Information Processing Systems - Volume 1, Curran Associates Inc., USA, NIPS'12, pp 1097-1105, event-place: Lake Tahoe, Nevada

Lauterbur PC (1973) Image Formation by Induced Local Interactions: Examples Employing Nuclear Magnetic Resonance. Nature 242(5394):190-191, DOI 10.1038/242190a0

Lauterbur PC (1974) Magnetic resonance zeugmatography. Pure and Applied Chemistry 40(1-2):149 157, DOI 10.1351/pac197440010149

Law M, Yang S, Wang H, Babb JS, Johnson G, Cha S, Knopp EA, Zagzag D (2003) Glioma grading: sensitivity, specificity, and predictive values of perfusion MR imaging and proton MR spectroscopic imaging compared with conventional MR imaging. AJNR American journal of neuroradiology 24(10):1989-1998

Law M, Young RJ, Babb JS, Peccerelli N, Chheang S, Gruber ML, Miller DC, Golfinos JG, Zagzag D, Johnson G (2008) Gliomas: predicting time to progression or survival with cerebral blood volume measurements at dynamic susceptibility-weighted contrast-enhanced perfusion MR imaging. Radiology 247(2):490-498, DOI 10.1148/radiol.2472070898

LeCun Y, Bengio Y, Hinton G (2015) Deep learning. Nature 521(7553):436-444, DOI 10.1038/ nature14539

Lee CH, Wang S, Murtha A, Brown MRG, Greiner R (2008) Segmenting brain tumors using pseudoconditional random fields. Medical image computing and computer-assisted intervention: MICCAI International Conference on Medical Image Computing and Computer-Assisted Intervention 11(Pt 1):359-366 
Lee J, Narang S, Martinez J, Rao G, Rao A (2015) Spatial Habitat Features Derived from Multiparametric Magnetic Resonance Imaging Data Are Associated with Molecular Subtype and 12-Month Survival Status in Glioblastoma Multiforme. PLOS ONE 10(9):e0136,557, DOI 10.1371/journal.pone.0136557

Lemée JM, Clavreul A, Menei P (2015) Intratumoral heterogeneity in glioblastoma: don't forget the peritumoral brain zone. Neuro-Oncology 17(10):1322-1332, DOI 10.1093/neuonc/nov119

Levine AB, Schlosser C, Grewal J, Coope R, Jones SJM, Yip S (2019) Rise of the Machines: Advances in Deep Learning for Cancer Diagnosis. Trends in Cancer 5(3):157-169, DOI 10.1016/j.trecan.2019. 02.002

Liu TT, Achrol AS, Mitchell LA, Rodriguez SA, Feroze A, Iv M, Kim C, Chaudhary N, Gevaert O, Stuart JM, Harsh GR, Chang SD, Rubin DL (2017a) Magnetic resonance perfusion image features uncover an angiogenic subgroup of glioblastoma patients with poor survival and better response to antiangiogenic treatment. Neuro-Oncology 19(7):997-1007, DOI 10.1093/neuonc/now270

Liu Y, Xu X, Yin L, Zhang X, Li L, Lu H (2017b) Relationship between Glioblastoma Heterogeneity and Survival Time: An MR Imaging Texture Analysis. AJNR American journal of neuroradiology 38(9):1695-1701, DOI 10.3174/ajnr.A5279

Lloyd S (1982) Least squares quantization in PCM. IEEE Transactions on Information Theory 28(2):129-137, DOI 10.1109/TIT.1982.1056489

Long J, Shelhamer E, Darrell T (2015) Fully Convolutional Networks for Semantic Segmentation. arXiv:14114038 [cs] ArXiv: 1411.4038

Louis DN, Perry A, Reifenberger G, von Deimling A, Figarella-Branger D, Cavenee WK, Ohgaki H, Wiestler OD, Kleihues P, Ellison DW (2016) The 2016 World Health Organization Classification of Tumors of the Central Nervous System: a summary. Acta Neuropathologica 131(6):803-820, DOI 10.1007\/s00401-016-1545-1

Lupo JM, Cha S, Chang SM, Nelson SJ (2005) Dynamic susceptibility-weighted perfusion imaging of high-grade gliomas: characterization of spatial heterogeneity. AJNR American journal of neuroradiology 26(6):1446-1454

Macqueen J (1967) Some methods for classification and analysis of multivariate observations. In: In 5-th Berkeley Symposium on Mathematical Statistics and Probability, pp 281-297

Mangla R, Singh G, Ziegelitz D, Milano MT, Korones DN, Zhong J, Ekholm SE (2010) Changes in relative cerebral blood volume 1 month after radiation-temozolomide therapy can help predict overall survival in patients with glioblastoma. Radiology 256(2):575-584, DOI 10.1148/radiol.10091440

Manjón JV, Coupé P, Buades A, Collins DL, Robles M (2010a) MRI superresolution using selfsimilarity and image priors. International Journal of Biomedical Imaging 2010:425,891, DOI 10. $1155 / 2010 / 425891$

Manjón JV, Coupé P, Buades A, Fonov V, Louis Collins D, Robles M (2010b) Non-local MRI upsampling. Medical Image Analysis 14(6):784-792, DOI 10.1016/j.media.2010.05.010

Manjón JV, Coupé P, Martí-Bonmatí L, Collins DL, Robles M (2010c) Adaptive non-local means denoising of MR images with spatially varying noise levels. Journal of magnetic resonance imaging: JMRI 31(1):192-203, DOI 10.1002/jmri.22003

Mansfield P (1977) Multi-planar image formation using NMR spin echoes. Journal of Physics C: Solid State Physics 10(3):L55-L58, DOI 10.1088/0022-3719/10/3/004 
Martin D, Fowlkes C, Tal D, Malik J (2001) A database of human segmented natural images and its application to evaluating segmentation algorithms and measuring ecological statistics. In: Proc. 8th Int'l Conf. Computer Vision, vol 2, pp 416-423

McCulloch WS, Pitts W (1943) A logical calculus of the ideas immanent in nervous activity. The bulletin of mathematical biophysics 5(4):115-133, DOI 10.1007/BF02478259

McRobbie DW, Moore EA, Graves MJ, Prince MR (2007) MRI from Picture to Proton, edición: 2 edn. Cambridge University Press, Cambridge, UK ; New York

Meier P, Zierler KL (1954) On the Theory of the Indicator-Dilution Method for Measurement of Blood Flow and Volume. Journal of Applied Physiology 6(12):731-744, DOI 10.1152/jappl.1954.6.12.731

Meier R, Bauer S, Slotboom J, Wiest R, Reyes M (2013) A hybrid model for multimodal brain tumor segmentation. Proceedings of NCI-MICCAI BRATS 2013 1(1):31-37

Menze BH, Jakab A, Bauer S, Kalpathy-Cramer J, Farahani K, Kirby J, Burren Y, Porz N, Slotboom J, Wiest R, Lanczi L, Gerstner E, Weber MA, Arbel T, Avants BB, Ayache N, Buendia P, Collins DL, Cordier N, Corso JJ, Criminisi A, Das T, Delingette H, Demiralp a, Durst CR, Dojat M, Doyle S, Festa J, Forbes F, Geremia E, Glocker B, Golland P, Guo X, Hamamci A, Iftekharuddin KM, Jena R, John NM, Konukoglu E, Lashkari D, Mariz JA, Meier R, Pereira S, Precup D, Price SJ, Raviv TR, Reza SMS, Ryan M, Sarikaya D, Schwartz L, Shin HC, Shotton J, Silva CA, Sousa N, Subbanna NK, Szekely G, Taylor TJ, Thomas OM, Tustison NJ, Unal G, Vasseur F, Wintermark M, Ye DH, Zhao L, Zhao B, Zikic D, Prastawa M, Reyes M, Van Leemput K (2015) The Multimodal Brain Tumor Image Segmentation Benchmark (BRATS). IEEE transactions on medical imaging 34(10):1993-2024, DOI 10.1109/TMI.2014.2377694

Neal R, Hinton G (1999) A View Of The Em Algorithm That Justifies Incremental, Sparse, And Other Variants. Learning in graphical models 89, DOI 10.1007/978-94-011-5014-9-12

Nie J, Xue Z, Liu T, Young GS, Setayesh K, Guo L, Wong STC (2009) Automated brain tumor segmentation using spatial accuracy-weighted hidden Markov Random Field. Computerized Medical Imaging and Graphics: The Official Journal of the Computerized Medical Imaging Society 33(6):431-441, DOI 10.1016/j.compmedimag.2009.04.006

Nikou C, Galatsanos NP, Likas AC (2007) A Class-Adaptive Spatially Variant Mixture Model for Image Segmentation. IEEE Transactions on Image Processing 16(4):1121-1130, DOI 10.1109/TIP. 2007.891771

Nikou C, Likas AC, Galatsanos NP (2010) A Bayesian Framework for Image Segmentation With Spatially Varying Mixtures. IEEE Transactions on Image Processing 19(9):2278-2289, DOI 10. 1109/TIP.2010.2047903

O'Connor JPB, Aboagye EO, Adams JE, Aerts HJWL, Barrington SF, Beer AJ, Boellaard R, Bohndiek SE, Brady M, Brown G, Buckley DL, Chenevert TL, Clarke LP, Collette S, Cook GJ, deSouza NM, Dickson JC, Dive C, Evelhoch JL, Faivre-Finn C, Gallagher FA, Gilbert FJ, Gillies RJ, Goh V, Griffiths JR, Groves AM, Halligan S, Harris AL, Hawkes DJ, Hoekstra OS, Huang EP, Hutton BF, Jackson EF, Jayson GC, Jones A, Koh DM, Lacombe D, Lambin P, Lassau N, Leach MO, Lee TY, Leen EL, Lewis JS, Liu Y, Lythgoe MF, Manoharan P, Maxwell RJ, Miles KA, Morgan B, Morris S, Ng T, Padhani AR, Parker GJM, Partridge M, Pathak AP, Peet AC, Punwani S, Reynolds AR, Robinson SP, Shankar LK, Sharma RA, Soloviev D, Stroobants S, Sullivan DC, Taylor SA, Tofts PS, Tozer GM, van Herk M, Walker-Samuel S, Wason J, Williams KJ, Workman P, Yankeelov TE, Brindle KM, McShane LM, Jackson A, Waterton JC (2017) Imaging biomarker roadmap for cancer studies. Nature Reviews Clinical Oncology 14(3):169-186, DOI 10.1038/nrclinonc.2016.162 
Ortiz-Pla J, Fuster-Garcia E, Juan-Albarracin J, Garcia-Gomez JM (2016) GBM Modeling with Proliferation and Migration Phenotypes: A Proposal of Initialization for Real Cases. In: Tsaftaris SA, Gooya A, Frangi AF, Prince JL (eds) Simulation and Synthesis in Medical Imaging, Springer International Publishing, Lecture Notes in Computer Science, pp 65-74

Ostergaard L, Weisskoff RM, Chesler DA, Gyldensted C, Rosen BR (1996) High resolution measurement of cerebral blood flow using intravascular tracer bolus passages. Part I: Mathematical approach and statistical analysis. Magnetic Resonance in Medicine 36(5):715-725, DOI $10.1002 / \mathrm{mrm} .1910360510$

Ostrom QT, Gittleman H, Fulop J, Liu M, Blanda R, Kromer C, Wolinsky Y, Kruchko C, BarnholtzSloan JS (2015) CBTRUS Statistical Report: Primary Brain and Central Nervous System Tumors Diagnosed in the United States in 2008-2012. Neuro-Oncology 17 Suppl 4:iv1-iv62, DOI 10.1093/ neuonc/nov189

Pal NR, Pal SK (1993) A review on image segmentation techniques. Pattern Recognition 26(9):12771294, DOI 10.1016/0031-3203(93)90135-J

Parisi G (1998) Statistical Field Theory, edición: 1 edn. CRC Press, Reading, Mass

Parsons DW, Jones S, Zhang X, Lin JCH, Leary RJ, Angenendt P, Mankoo P, Carter H, Siu IM, Gallia GL, Olivi A, McLendon R, Rasheed BA, Keir S, Nikolskaya T, Nikolsky Y, Busam DA, Tekleab H, Diaz LA, Hartigan J, Smith DR, Strausberg RL, Marie SKN, Shinjo SMO, Yan H, Riggins GJ, Bigner DD, Karchin R, Papadopoulos N, Parmigiani G, Vogelstein B, Velculescu VE, Kinzler KW (2008) An integrated genomic analysis of human glioblastoma multiforme. Science (New York, NY) 321(5897):1807-1812, DOI 10.1126/science.1164382

Pillonetto G, De Nicolao G (2010) A new kernel-based approach for linear system identification. Automatica 46(1):81-93, DOI 10.1016/j.automatica.2009.10.031

Plenge E, Poot DHJ, Niessen WJ, Meijering E (2013) Super-resolution reconstruction using crossscale self-similarity in multi-slice MRI. Medical image computing and computer-assisted intervention: MICCAI International Conference on Medical Image Computing and Computer-Assisted Intervention 16(Pt 3):123-130, DOI 10.1007/978-3-642-40760-4\_16

Prastawa M, Bullitt E, Moon N, Van Leemput K, Gerig G (2003) Automatic brain tumor segmentation by subject specific modification of atlas priors. Academic Radiology 10(12):1341-1348, DOI 10. 1016/s1076-6332(03)00506-3

Price SJ, Young AMH, Scotton WJ, Ching J, Mohsen LA, Boonzaier NR, Lupson VC, Griffiths JR, McLean MA, Larkin TJ (2016) Multimodal MRI can identify perfusion and metabolic changes in the invasive margin of glioblastomas. Journal of magnetic resonance imaging: JMRI 43(2):487-494, DOI 10.1002/jmri.24996

Protter M, Elad M, Takeda H, Milanfar P (2009) Generalizing the Nonlocal-Means to SuperResolution Reconstruction. IEEE Transactions on Image Processing 18(1):36-51, DOI 10.1109/ TIP.2008.2008067

Raschke F, Barrick TR, Jones TL, Yang G, Ye X, Howe FA (2019) Tissue-type mapping of gliomas. NeuroImage: Clinical 21:101,648, DOI 10.1016/j.nicl.2018.101648

Rempp KA, Brix G, Wenz F, Becker CR, Gückel F, Lorenz WJ (1994) Quantification of regional cerebral blood flow and volume with dynamic susceptibility contrast-enhanced MR imaging. Radiology 193(3):637-641, DOI 10.1148/radiology.193.3.7972800

Reza S, Iftekharuddin K (2013) Multi-class abnormal brain tissue segmentation using texture features. Proceedings of NCI-MICCAI BRATS 2013 1(1):38-42 
Röntgen WC (1898) Ueber eine neue Art von Strahlen. Annalen der Physik 300(1):12-17, DOI 10.1002/andp.18983000103

Rokach L, Maimon O (2005) Clustering Methods. In: Maimon O, Rokach L (eds) Data Mining and Knowledge Discovery Handbook, Springer US, Boston, MA, pp 321-352, DOI 10.1007/ 0-387-25465-X $\backslash \_15$

Ronneberger O, Fischer P, Brox T (2015) U-Net: Convolutional Networks for Biomedical Image Segmentation. In: Navab N, Hornegger J, Wells WM, Frangi AF (eds) Medical Image Computing and Computer-Assisted Intervention - MICCAI 2015, Springer International Publishing, Lecture Notes in Computer Science, pp 234-241

Rosenblatt F (1958) The Perceptron: A Probabilistic Model for Information Storage and Organization in The Brain. Psychological Review pp 65-386

Rousseau F, Alzheimer's Disease Neuroimaging Initiative (2010) A non-local approach for image super-resolution using intermodality priors. Medical Image Analysis 14(4):594-605, DOI 10.1016/ j.media.2010.04.005

Ruan S, Zhang N, Liao Q, Zhu Y (2011) Image fusion for following-up brain tumor evolution. In: 2011 IEEE International Symposium on Biomedical Imaging: From Nano to Macro, pp 281-284, DOI 10.1109/ISBI.2011.5872406

Rudie JD, Rauschecker AM, Bryan RN, Davatzikos C, Mohan S (2019) Emerging Applications of Artificial Intelligence in Neuro-Oncology. Radiology 290(3):607-618, DOI 10.1148/radiol.2018181928

Rumelhart DE, Hinton GE, Williams RJ (1986) Learning representations by back-propagating errors. Nature 323(6088):533-536, DOI 10.1038/323533a0

Russell S, Norvig P (2016) Artificial Intelligence: A Modern Approach, Global Edition, edición: 3 edn. Pearson

Saman S, Jamjala Narayanan S (2019) Survey on brain tumor segmentation and feature extraction of MR images. International Journal of Multimedia Information Retrieval 8(2):79-99, DOI 10.1007/ s13735-018-0162-2

Sanjay-Gopal S, Hebert TJ (1998) Bayesian pixel classification using spatially variant finite mixtures and the generalized EM algorithm. IEEE Transactions on Image Processing 7(7):1014-1028, DOI $10.1109 / 83.701161$

Sanz-Requena R, Revert-Ventura A, Martí-Bonmatí L, Alberich-Bayarri A, García-Martí G (2013) Quantitative MR perfusion parameters related to survival time in high-grade gliomas. European Radiology 23(12):3456-3465, DOI 10.1007/s00330-013-2967-y

Sawlani RN, Raizer J, Horowitz SW, Shin W, Grimm SA, Chandler JP, Levy R, Getch C, Carroll TJ (2010) Glioblastoma: a method for predicting response to antiangiogenic chemotherapy by using MR perfusion imaging-pilot study. Radiology 255(2):622-628, DOI 10.1148/radiol.10091341

Saxena A, Prasad M, Gupta A, Bharill N, Patel OP, Tiwari A, Er MJ, Ding W, Lin CT (2017) A review of clustering techniques and developments. Neurocomputing 267:664-681, DOI 10.1016/j. neucom.2017.06.053

Schaefer PW, Grant PE, Gonzalez RG (2000) Diffusion-weighted MR imaging of the brain. Radiology 217(2):331-345, DOI 10.1148/radiology.217.2.r00nv24331

Schmid VJ (2011) Voxel-based adaptive spatio-temporal modelling of perfusion cardiovascular MRI. IEEE transactions on medical imaging 30(7):1305-1313, DOI 10.1109/TMI.2011.2109733 
Schmid VJ, Gatehouse PD, Yang GZ (2007) Attenuation Resilient AIF Estimation Based on Hierarchical Bayesian Modelling for First Pass Myocardial Perfusion MRI. In: Ayache N, Ourselin S, Maeder A (eds) Medical Image Computing and Computer-Assisted Intervention - MICCAI 2007, Springer Berlin Heidelberg, Lecture Notes in Computer Science, pp 393-400

Schnack HG, van Haren NEM, Hulshoff Pol HE, Picchioni M, Weisbrod M, Sauer H, Cannon T, Huttunen M, Murray R, Kahn RS (2004) Reliability of brain volumes from multicenter MRI acquisition: a calibration study. Human Brain Mapping 22(4):312-320, DOI 10.1002/hbm.20040

Sáez C, Robles M, García-Gómez JM (2017) Stability metrics for multi-source biomedical data based on simplicial projections from probability distribution distances. Statistical Methods in Medical Research 26(1):312-336, DOI 10.1177/0962280214545122

Sfikas G, Nikou C, Galatsanos N (2008) Edge preserving spatially varying mixtures for image segmentation. In: 2008 IEEE Conference on Computer Vision and Pattern Recognition, pp 1-7, DOI 10.1109/CVPR.2008.4587416

Shah MK, Shin W, Parikh VS, Ragin A, Mouannes J, Bernstein RA, Walker MT, Bhatt H, Carroll TJ (2010) Quantitative cerebral MR perfusion imaging: preliminary results in stroke. Journal of magnetic resonance imaging: JMRI 32(4):796-802, DOI 10.1002/jmri.22302

Sled JG, Zijdenbos AP, Evans AC (1998) A nonparametric method for automatic correction of intensity nonuniformity in MRI data. IEEE transactions on medical imaging 17(1):87-97, DOI $10.1109 / 42.668698$

Soeda A, Hara A, Kunisada T, Yoshimura Si, Iwama T, Park DM (2015) The evidence of glioblastoma heterogeneity. Scientific Reports 5:7979, DOI 10.1038/srep07979

Soltaninejad M, Zhang L, Lambrou T, Yang G, Allinson N, Ye X (2018) MRI Brain Tumor Segmentation and Patient Survival Prediction Using Random Forests and Fully Convolutional Networks. In: Crimi A, Bakas S, Kuijf H, Menze B, Reyes M (eds) Brainlesion: Glioma, Multiple Sclerosis, Stroke and Traumatic Brain Injuries, Springer International Publishing, Lecture Notes in Computer Science, pp 204-215

Østergaard L (2005) Principles of cerebral perfusion imaging by bolus tracking. Journal of magnetic resonance imaging: JMRI 22(6):710-717, DOI 10.1002/jmri.20460

Stupp R, Mason WP, van den Bent MJ, Weller M, Fisher B, Taphoorn MJB, Belanger K, Brandes AA, Marosi C, Bogdahn U, Curschmann J, Janzer RC, Ludwin SK, Gorlia T, Allgeier A, Lacombe D, Cairncross JG, Eisenhauer E, Mirimanoff RO, European Organisation for Research and Treatment of Cancer Brain Tumor and Radiotherapy Groups, National Cancer Institute of Canada Clinical Trials Group (2005) Radiotherapy plus concomitant and adjuvant temozolomide for glioblastoma. The New England Journal of Medicine 352(10):987-996, DOI 10.1056/NEJMoa043330

Svolos P, Kousi E, Kapsalaki E, Theodorou K, Fezoulidis I, Kappas C, Tsougos I (2014) The role of diffusion and perfusion weighted imaging in the differential diagnosis of cerebral tumors: a review and future perspectives. Cancer Imaging: The Official Publication of the International Cancer Imaging Society 14:20, DOI 10.1186/1470-7330-14-20

Tamimi AF, Juweid M (2017) Epidemiology and Outcome of Glioblastoma. In: De Vleeschouwer S (ed) Glioblastoma, Codon Publications, Brisbane (AU)

Tandel GS, Biswas M, Kakde OG, Tiwari A, Suri HS, Turk M, Laird JR, Asare CK, Ankrah AA, Khanna NN, Madhusudhan BK, Saba L, Suri JS (2019) A Review on a Deep Learning Perspective in Brain Cancer Classification. Cancers 11(1), DOI 10.3390/cancers11010111 
Thompson G, Mills SJ, Coope DJ, O'connor JPB, Jackson A (2011) Imaging biomarkers of angiogenesis and the microvascular environment in cerebral tumours. The British Journal of Radiology 84(Spec Iss 2):S127-S144, DOI 10.1259/bjr/66316279

Thompson Howard K, Starmer C Frank, Whalen Robert E, Mcintosh Henry D (1964) Indicator Transit Time Considered as a Gamma Variate. Circulation Research 14(6):502-515, DOI 10.1161/ 01.RES.14.6.502

Tofts P (2004) Quantitative MRI of the Brain: Measuring Changes Caused by Disease. Wiley John + Sons, Chichester, West Sussex

Álvarez Torres MDM, Juan-Albarracín J, Fuster-Garcia E, Bellvís-Bataller F, Lorente D, Reynés G, Font de Mora J, Aparici-Robles F, Botella C, Muñoz-Langa J, Faubel R, Asensio-Cuesta S, GarcíaFerrando GA, Chelebian E, Auger C, Pineda J, Rovira A, Oleaga L, Mollà-Olmos E, Revert AJ, Tshibanda L, Crisi G, Emblem KE, Martin D, Due-Tønnessen P, Meling TR, Filice S, Sáez C, García-Gómez JM (2019) Robust association between vascular habitats and patient prognosis in glioblastoma: An international multicenter study. Journal of magnetic resonance imaging: JMRI DOI 10.1002/jmri.26958

Tustison N, Wintermark M, Durst CR, Avants B (2013) ANTs and árboles. Proceedings of NCIMICCAI BRATS 2013 1(1):47-50

Tustison NJ, Avants BB, Cook PA, Zheng Y, Egan A, Yushkevich PA, Gee JC (2010) N4itk: improved N3 bias correction. IEEE transactions on medical imaging 29(6):1310-1320, DOI 10.1109/TMI. 2010.2046908

Tustison NJ, Cook PA, Klein A, Song G, Das SR, Duda JT, Kandel BM, van Strien N, Stone JR, Gee JC, Avants BB (2014) Large-scale evaluation of ANTs and FreeSurfer cortical thickness measurements. NeuroImage 99:166-179, DOI 10.1016/j.neuroimage.2014.05.044

Tykocinski ES, Grant RA, Kapoor GS, Krejza J, Bohman LE, Gocke TA, Chawla S, Halpern CH, Lopinto J, Melhem ER, O'Rourke DM (2012) Use of magnetic perfusion-weighted imaging to determine epidermal growth factor receptor variant III expression in glioblastoma. Neuro-Oncology 14(5):613-623, DOI 10.1093/neuonc/nos073

Uematsu H, Maeda M, Sadato N, Matsuda T, Ishimori Y, Koshimoto Y, Kimura H, Yamada H, Kawamura Y, Yonekura Y, Itoh H (2001) Blood volume of gliomas determined by double-echo dynamic perfusion-weighted MR imaging: a preliminary study. AJNR American journal of neuroradiology 22(10):1915-1919

Ulyte A, Katsaros VK, Liouta E, Stranjalis G, Boskos C, Papanikolaou N, Usinskiene J, Bisdas S (2016) Prognostic value of preoperative dynamic contrast-enhanced MRI perfusion parameters for high-grade glioma patients. Neuroradiology 58(12):1197-1208, DOI 10.1007/s00234-016-1741-7

Verhaak RGW, Hoadley KA, Purdom E, Wang V, Qi Y, Wilkerson MD, Miller CR, Ding L, Golub T, Mesirov JP, Alexe G, Lawrence M, O’Kelly M, Tamayo P, Weir BA, Gabriel S, Winckler W, Gupta S, Jakkula L, Feiler HS, Hodgson JG, James CD, Sarkaria JN, Brennan C, Kahn A, Spellman PT, Wilson RK, Speed TP, Gray JW, Meyerson M, Getz G, Perou CM, Hayes DN, Cancer Genome Atlas Research Network (2010) Integrated genomic analysis identifies clinically relevant subtypes of glioblastoma characterized by abnormalities in PDGFRA, IDH1, EGFR, and NF1. Cancer Cell 17(1):98-110, DOI 10.1016/j.ccr.2009.12.020

Verma R, Zacharaki EI, Ou Y, Cai H, Chawla S, Lee SK, Melhem ER, Wolf R, Davatzikos C (2008) Multiparametric tissue characterization of brain neoplasms and their recurrence using pattern classification of MR images. Academic Radiology 15(8):966-977, DOI 10.1016/j.acra.2008.01.029 
Vidiri A, Pace A, Fabi A, Maschio M, Latagliata GM, Anelli V, Piludu F, Carapella CM, Giovinazzo G, Marzi S (2012) Early perfusion changes in patients with recurrent high-grade brain tumor treated with Bevacizumab: preliminary results by a quantitative evaluation. Journal of experimental \& clinical cancer research: CR 31:33, DOI 10.1186/1756-9966-31-33

Vijayakumar C, Damayanti G, Pant R, Sreedhar CM (2007) Segmentation and grading of brain tumors on apparent diffusion coefficient images using self-organizing maps. Computerized Medical Imaging and Graphics: The Official Journal of the Computerized Medical Imaging Society 31(7):473-484, DOI 10.1016/j.compmedimag.2007.04.004

Virchow RLK (2018) Die Krankhaften Geschwulste. Wentworth Press

Vonken EP, Beekman FJ, Bakker CJ, Viergever MA (1999) Maximum likelihood estimation of cerebral blood flow in dynamic susceptibility contrast MRI. Magnetic Resonance in Medicine 41(2):343-350, DOI 10.1002/(sici)1522-2594(199902)41:2〈343::aid-mrm19〉3.0.co;2-t

Vonken EP, van Osch MJ, Bakker CJ, Viergever MA (2000) Simultaneous quantitative cerebral perfusion and Gd-DTPA extravasation measurement with dual-echo dynamic susceptibility contrast MRI. Magnetic Resonance in Medicine 43(6):820-827, DOI 10.1002/1522-2594(200006)43:6<820:: aid-mrm7 $>3.0 . c o ; 2-f$

Wadhwa A, Bhardwaj A, Singh Verma V (2019) A review on brain tumor segmentation of MRI images. Magnetic Resonance Imaging 61:247-259, DOI 10.1016/j.mri.2019.05.043

Wangaryattawanich P, Hatami M, Wang J, Thomas G, Flanders A, Kirby J, Wintermark M, Huang ES, Bakhtiari AS, Luedi MM, Hashmi SS, Rubin DL, Chen JY, Hwang SN, Freymann J, Holder CA, Zinn PO, Colen RR (2015) Multicenter imaging outcomes study of The Cancer Genome Atlas glioblastoma patient cohort: imaging predictors of overall and progression-free survival. NeuroOncology 17(11):1525-1537, DOI 10.1093/neuonc/nov117

Weis SM, Cheresh DA (2011) Tumor angiogenesis: molecular pathways and therapeutic targets. Nature Medicine 17(11):1359-1370, DOI 10.1038/nm.2537

Weisskoff RM, Chesler D, Boxerman JL, Rosen BR (1993) Pitfalls in MR measurement of tissue blood flow with intravascular tracers: which mean transit time? Magnetic Resonance in Medicine 29(4):553-558, DOI 10.1002/mrm.1910290420

Weisskoff RM, Boxerman JL, Sorensen AG (1994) Simultaneous blood volume and permeability mapping using a single Gd-based contrast injection. In: Proceedings of the Society of Magnetic Resonance, Second Annual Meeting, Berkeley. Society of Magnetic Resonance, p 279

Wen PY, Macdonald DR, Reardon DA, Cloughesy TF, Sorensen AG, Galanis E, Degroot J, Wick W, Gilbert MR, Lassman AB, Tsien C, Mikkelsen T, Wong ET, Chamberlain MC, Stupp R, Lamborn KR, Vogelbaum MA, van den Bent MJ, Chang SM (2010) Updated response assessment criteria for high-grade gliomas: response assessment in neuro-oncology working group. Journal of Clinical Oncology: Official Journal of the American Society of Clinical Oncology 28(11):1963-1972, DOI 10.1200/JCO.2009.26.3541

Wetzel SG, Cha S, Johnson G, Lee P, Law M, Kasow DL, Pierce SD, Xue X (2002) Relative cerebral blood volume measurements in intracranial mass lesions: interobserver and intraobserver reproducibility study. Radiology 224(3):797-803, DOI 10.1148/radiol.2243011014

Wittek P (2014) 5 - Unsupervised Learning. In: Wittek P (ed) Quantum Machine Learning, Academic Press, Boston, pp 57-62, DOI 10.1016/B978-0-12-800953-6.00005-0

Woolrich MW, Behrens TEJ, Beckmann CF, Smith SM (2005) Mixture models with adaptive spatial regularization for segmentation with an application to FMRI data. IEEE Transactions on Medical Imaging 24(1):1-11, DOI 10.1109/TMI.2004.836545 
Wu CFJ (1983) On the Convergence Properties of the EM Algorithm. The Annals of Statistics 11(1):95-103, DOI 10.1214/aos/1176346060

Wu O, Østergaard L, Weisskoff RM, Benner T, Rosen BR, Sorensen AG (2003) Tracer arrival timing-insensitive technique for estimating flow in MR perfusion-weighted imaging using singular value decomposition with a block-circulant deconvolution matrix. Magnetic Resonance in Medicine 50(1):164-174, DOI 10.1002/mrm.10522

Wu Y, Tracey B, Natarajan P, Noonan JP (2013) Probabilistic Non-Local Means. IEEE Signal Processing Letters 20(8):763-766, DOI 10.1109/LSP.2013.2263135

Yankeelov TE, Pickens DR, Price RR (2011) Quantitative MRI in Cancer, edición: 1 edn. CRC Press, Boca Raton, FL

Yedidia JS, Freeman WT, Weiss Y (2003) Understanding belief propagation and its generalizations. In: Exploring artificial intelligence in the new millennium, Morgan Kaufmann Publishers Inc., pp $239-269$

Young R, Babb J, Law M, Pollack E, Johnson G (2007) Comparison of region-of-interest analysis with three different histogram analysis methods in the determination of perfusion metrics in patients with brain gliomas. Journal of magnetic resonance imaging: JMRI 26(4):1053-1063, DOI 10.1002/ jmri.21064

Zanderigo F, Bertoldo A, Pillonetto G, Cobelli Ast C (2009) Nonlinear stochastic regularization to characterize tissue residue function in bolus-tracking MRI: assessment and comparison with SVD, block-circulant SVD, and Tikhonov. IEEE transactions on bio-medical engineering 56(5):12871297, DOI 10.1109/TBME.2009.2013820

Zhang H, Fritts JE, Goldman SA (2008) Image segmentation evaluation: A survey of unsupervised methods. Computer Vision and Image Understanding 110(2):260-280, DOI 10.1016/j.cviu.2007.08. 003

Zhang Y, Brady M, Smith S (2001) Segmentation of brain MR images through a hidden Markov random field model and the expectation-maximization algorithm. IEEE transactions on medical imaging 20(1):45-57, DOI 10.1109/42.906424

Zhou M, Hall L, Goldgof D, Russo R, Balagurunathan Y, Gillies R, Gatenby R (2014) Radiologically Defined Ecological Dynamics and Clinical Outcomes in Glioblastoma Multiforme: Preliminary Results. Translational Oncology 7(1):5-13, DOI 10.1593/tlo.13730

Zhou M, Chaudhury B, Hall LO, Goldgof DB, Gillies RJ, Gatenby RA (2017) Identifying spatial imaging biomarkers of glioblastoma multiforme for survival group prediction. Journal of magnetic resonance imaging: JMRI 46(1):115-123, DOI 10.1002/jmri.25497

Zhu Y, Young GS, Xue Z, Huang RY, You H, Setayesh K, Hatabu H, Cao F, Wong ST (2012) Semiautomatic segmentation software for quantitative clinical brain glioblastoma evaluation. Academic Radiology 19(8):977-985, DOI 10.1016/j.acra.2012.03.026

Zierler Kenneth L (1962) Theoretical Basis of Indicator-Dilution Methods For Measuring Flow and Volume. Circulation Research 10(3):393-407, DOI 10.1161/01.RES.10.3.393 